\title{
Signaling microdomains within the myoendothelial junction
}

\author{
Lauren Alysse Biwer \\ Casa Grande, Arizona \\ Master of Science in Biological and Physical Sciences \\ University of Virginia, 2014 \\ Bachelor of Science in Health Science Studies \\ Baylor University, 2009
}

A Dissertation presented to the Graduate Faculty of the University of Virginia in Candidacy for the Degree of Doctor of Philosophy

Department of Molecular Physiology and Biological Physics

University of Virginia

June 2017 


\section{$\underline{\text { Abstract }}$}

Communication between endothelial and smooth muscle cells is critical to maintain homeostatic vascular tone and blood pressure. In resistance arteries, the cells make direct cytoplasmic contact via cellular extensions that project through the thin extracellular matrix of the internal elastic lamina that separates them. These structures are termed myoendothelial junctions (MEJ). There are a number of proteins and signaling processes localized to this part of the arterial wall that regulate bi-directional communication between the endothelium and smooth muscle. In particular, a number of proteins localized to the MEJ mediate calcium signaling and there is localized calcium release from the endoplasmic reticulum via the inositol $(1,4,5)$ trisphosphate receptor that can influence vascular tone and negative feedback. There is a detailed description of the creation and isolation of in vitro MEJ using the vascular cell co-culture technique, which has allowed for deeper exploration of signaling molecules and processes occurring at the MEJ. Smooth muscle cells and endothelial cells grow on opposite sides of a filter inset, and the MEJ form within the holes of the filter. This model can be used to immunofluorescently label proteins or the cellular fractions can be individually isolated to investigate protein expression and phosphorylation via western blotting. Using this unique model, we show the in vitro MEJ has a unique lipid composition that is rich in diacylglycerol and phosphatidylserine. This likely facilitates localization of signaling molecules such as protein kinase $\mathrm{C}$ and selective activation of endothelial nitric oxide synthase. Additionally, the multifunctional protein calreticulin is highly expressed at the MEJ of resistance arteries and endothelial deletion of calreticulin results in impaired MEJ calcium signaling, thus vasoconstriction to phenylephrine and blood pressure. Our data suggests that EC monolayer Calr could be 
functionally different than MEJ-localized Calr, which may be located outside the ER. Further work will need to be done in order to clarify and confirm MEJ Calr function and how it affects calcium signaling at the MEJ specifically in response to phenylephrine. In summary, the MEJ is an important signaling microdomain strategically located in the wall of resistance arteries to mediate heterocellular communication. 


\section{TABLE OF CONTENTS}

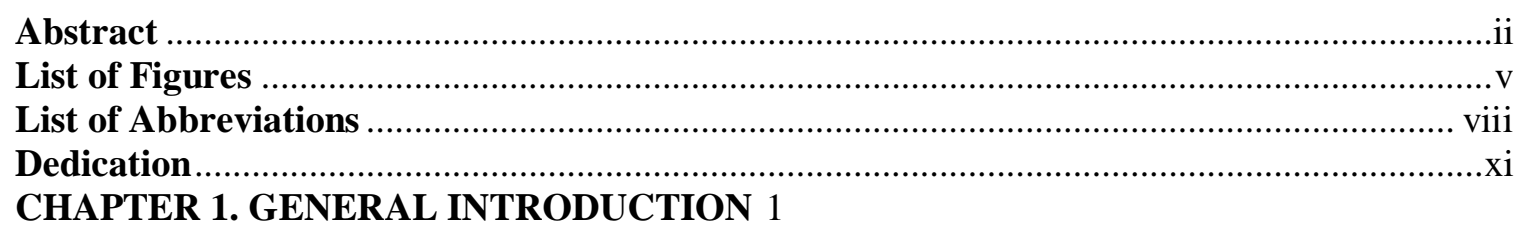

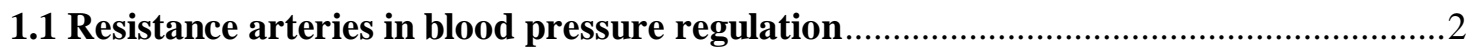

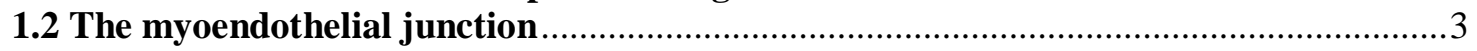

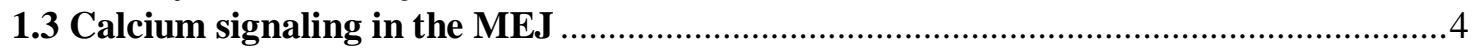

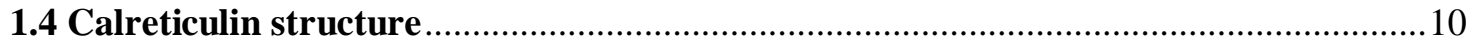

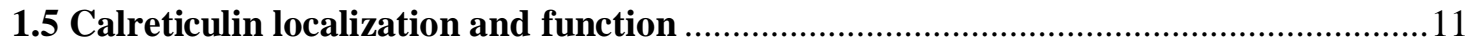

CHAPTER 2. THE ENDOPLASMIC RETICULUM AS A MEDIATOR OF CELLULAR

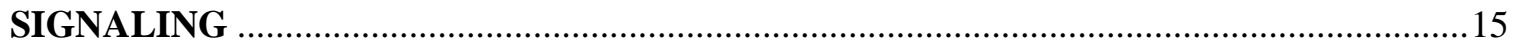

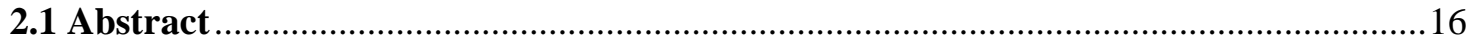

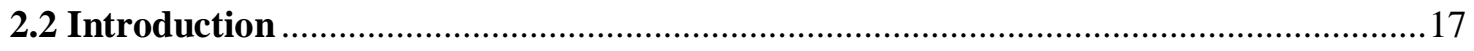

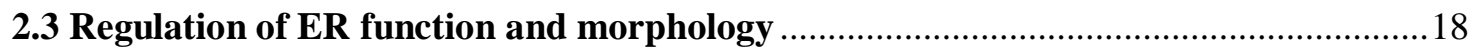

2.4 Lipid synthesis, trafficking and signaling centered around the ER ........................23

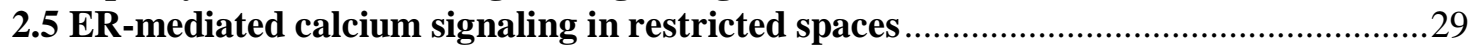

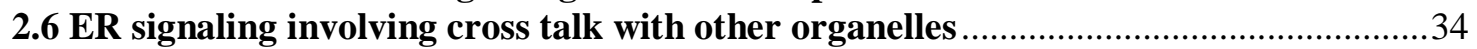

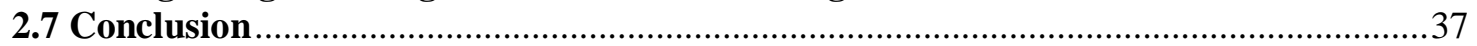

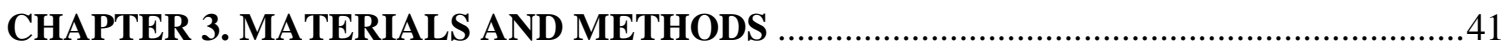

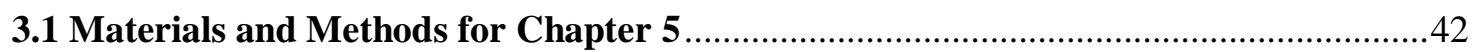

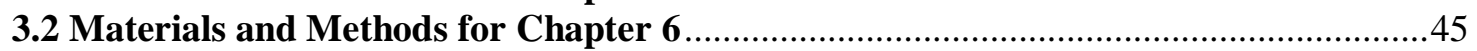

CHAPTER 4. A CELL CULTURE MODEL OF RESISTANCE ARTERIES …..................54

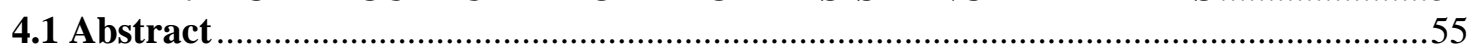

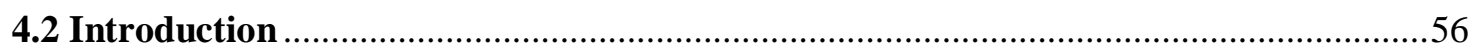

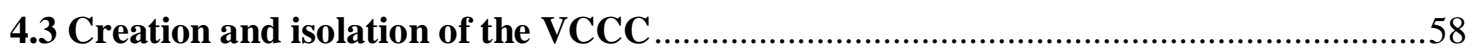

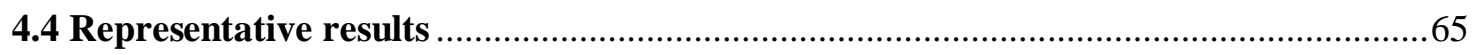

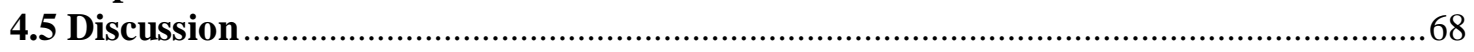

CHAPTER 5. THE MYOENDOTHELIAL JUNCTION HAS A UNIQUE LIPID

COMPOSITION THAT FACILITATES PROTEIN KINASE C ACTIVATION AND ENOS

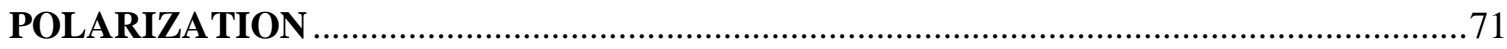

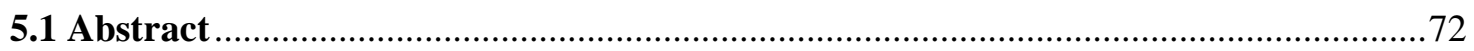

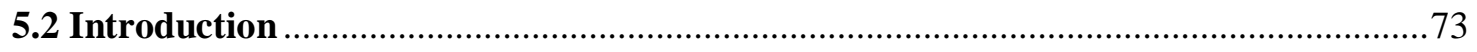

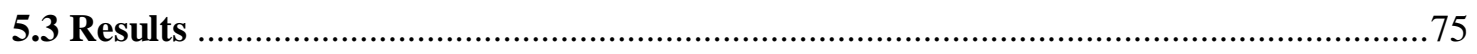

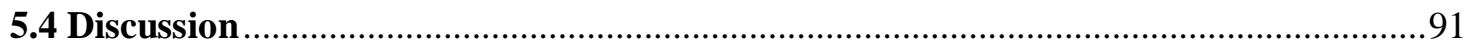

CHAPTER 6. CALRETICULIN LOCALIZED TO THE MYOENDOTHELIAL JUNCTION MEDIATES HETEROCELLULAR SIGNALING AND VASCULAR

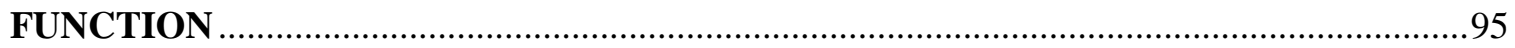

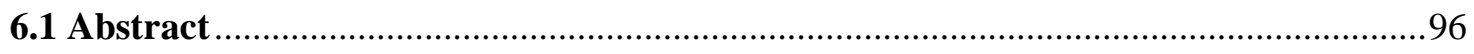

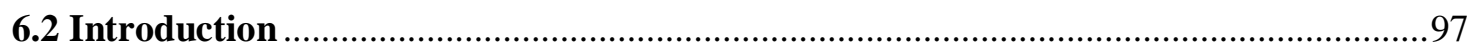

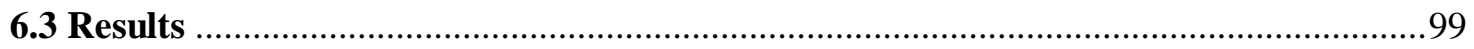

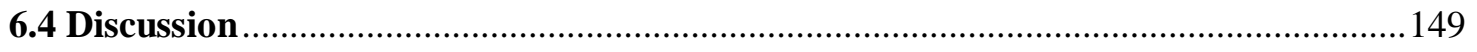

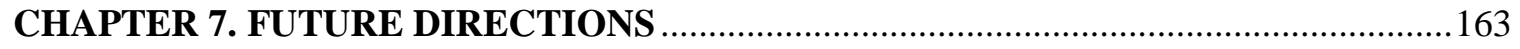

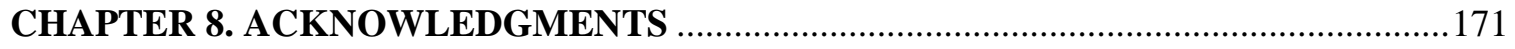

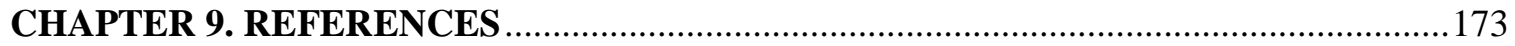




\section{LIST OF FIGURES}

\section{CHAPTER 2}

Figure 1. Endoplasmic reticulum (ER) localization and signaling in microdomains..........38

Figure 2. An example of ER in restricted spaces: the myoendothelial junction (MEJ).......40

\section{CHAPTER 4}

Figure 3. Plating of the vascular cell co-culture..........................................................66

Figure 4. Alpha globin and plasminogen activator inhibitor-1 expression in the MEJ.......67

\section{CHAPTER 5}

Figure 5. Endothelial nitric oxide synthase (eNOS) at Serine 1177 is selectively phosphorylated based upon endothelial cell (EC) or smooth muscle cell receptor activation.

Figure 6. Gap junction inhibition prevents phenylephrine (PE) induced eNOS phosphorylation at the MEJ

Figure 7. Diacylglycerol and phosphatidylserine are enriched at the MEJ, facilitating a significant increase in protein kinase $\mathrm{C}$ activity.....

Figure 8. Primary endothelial and smooth muscle cells grown in the VCCC have a unique lipid composition.

Figure 9. Endoplasmic reticulum is present at the MEJ, influencing eNOS phosphorylation. .86

Figure 10. Functional effects of apical versus MEJ eNOS

Figure 11. Proposed mechanism of differential eNOS phosphorylation in endothelium....89 


\section{CHAPTER 6}

Figure 12. Calreticulin (Calr) is enriched at the majority of holes in the arterial internal elastic lamina (IEL). 106

Figure 13. Quantification of calreticulin in EC and MEJ via immuno-transmission electron microscopy 108

Figure 14. Generation of EC specific, tamoxifen inducible Calr knockout mouse. 110

Figure 15. Representative flow cytometry plots for EC Calr fl/fl and EC Calr $\Delta / \Delta$ diaphragm and Calr mean fluorescence intensity

Figure 16. EC Calr $\Delta / \Delta$ does not change blood cell indices.

Figure 17. Representative trichrome and hemotoxylin/eosin stained arteries from EC Calr $\mathrm{fl} / \mathrm{fl}$ and EC Calr $\Delta / \Delta$ mice.

Figure 18. Relative expression of mRNA in thoracic aortas from EC Calr fl/fl and EC Calr $\Delta / \Delta$ mice. 116

Figure 19. Binding immunoglobulin protein expression after EC Calr knockout.

Figure 20. EC Calr $\Delta / \Delta$ third order mesenteric arteries have differential, IEL localized calcium responses to adrenergic versus muscarinic agonists.

Figure 21. Dilation to $10 \mu \mathrm{M} \mathrm{CCh}$ in EC Calr fl/fl and EC Calr $\Delta / \Delta$

Figure 22. EC Calr $\Delta / \Delta$ arteries have differential vasoactive responses to adrenergic versus muscarinic agonists that results in higher mean arterial pressure .121

Figure 23. Acute measurement of nighttime blood pressure

Figure 24. Acute blood pressure response to PE injection. 124 Figure 25. Time course of blood pressure response to angiotensin II 125 
Figure 26. EC Calr $\Delta / \Delta$ does not change EC independent dilation or SMC constriction to potassium chloride 127

Figure 27. Time course of average daily blood pressure and heart rate...........................128

Figure 28. Calr knockdown in EC does not affect the level of ER calcium......................130

Figure 29. GCaMP-ER flow cytometry gating strategy and representative results..........132

Figure 30. GCaMP-ER signal decreases after addition of ATP....................................134

Figure 31. Time course of Calr siRNA knockout efficiency........................................135

Figure 32. In vitro Calr co-immunoprecipitation shows differential interaction with

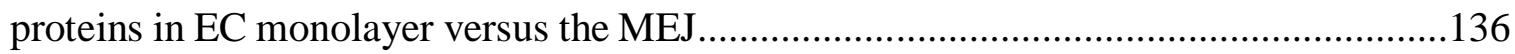

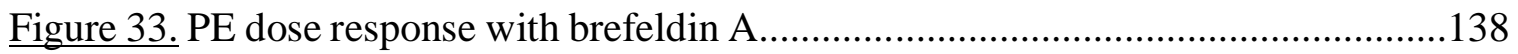

Figure 34. Calr at MEJ may be localized outside the ER.............................................139

Figure 35. Calcium signals in EC Calr $\Delta / \Delta$ arteries are diminished with

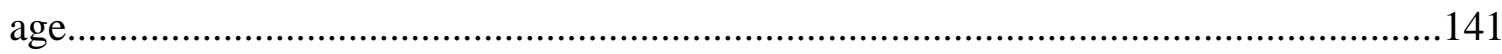

Figure 36. Aged EC Calr $\Delta / \Delta$ arteries have significantly diminished calcium responses to

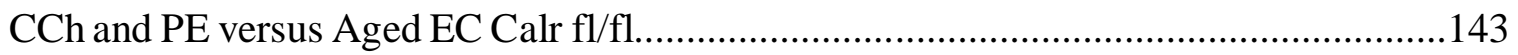
Figure 37. Inositol triphosphate kinase A interacts with Calr at the MEJ to mediate PE constriction. 145

Figure 38. Kif18B interacts with Calr at the MEJ but has no effect on PE constriction.....147 


\section{List of Abbreviations}

BiP/GRP78: binding immunoglobulin protein/glucose regulated protein 78

Calr: calreticulin

CaMKII: calcium calmodulin kinase II

CCh: carbachol

cDNA: copy DNA

CERT: ceramide transfer protein

cGMP: cyclic guanosine monophosphate

CICR: calcium induced calcium release

CPA: cyclopiazonic acid

Cx: connexin

EC: endothelial cell

eNOS: endothelial nitric oxide synthesis

ER: endoplasmic reticulum

HIEL: holes in the internal elastic lamina

IEL: internal elastic lamina

IK: intermediate conductance calcium activated potassium channel

$\mathrm{IP}_{3}$ : inositol $(1,4,5)$ trisphosphate

IP3R: inositol $(1,4,5)$ trisphosphate receptor

IP4: inositol $(1,3,4,5)$ tetrakisphosphate

ITPKA: inositol trisphosphate kinase A

KIF18B: kinesin-like factor $18 \mathrm{~b}$

Lnp1: lunapark 1 
MAM: mitochondrial associated matrix

MAP: mean arterial pressure

MEJ: myoendothelial junction

mRNA: messenger RNA

NO: nitric oxide

PAI-1: plasminogen activator inhibitor 1

PE: phenylephrine

PM: plasma membrane

qRT-PCR: quantitative real time polymerase chain reaction

rER: rough endoplasmic reticulum

RyR: ryanodine receptor

SCAP: SREBP cleavage activating protein

SERCA: sarco-endoplasmic reticulum calcium ATP-ase

sER: smooth endoplasmic reticulum

siRNA: small interfering RNA

SK: small conductance calcium activated potassium channel

SMC: smooth muscle cell

SR: sarcoplasmic reticulum

SREBP: sterol regulatory element binding protein

STIM1: stromal interaction molecule 1

TAC: tip attachment complex

TAT: trans-activator of transcription

TRPA1: transient receptor potential ankyrin 1 
TRPV4: transient receptor potential vanilloid 4

VCCC: Vascular cell co-culture model 


\section{Dedication}

Thank you first and foremost to my parents Mari and Jerry, who are my biggest fans and the best examples of hard work, dedication, passion, and not giving up. Thank you to Ian for all the love, encouragement, and adventures. Thank you to Arielle for always knowing how to push my buttons- the good and bad ones. Thank you to Grandma Ailene, can I retire and come hang out with you at the pool now? Lo-Lo, I know you can't read yet, but thank you for always making me smile.

Motivation comes from working on things you care about, but it also comes from working with people you care about. To my PhD advisor Dr. Brant Isakson, thank you for being you. You taught me to think outside the box, get out of my comfort zone and that enthusiastic persistence is key. I especially thank you for all the coffee and fantastic dinners along the way. To my former boss, Dr. Taben Hale, I definitely would not be here without you. Thank you for being an amazing role model. To my committee members (Dr. Avril Somylo, Dr. Thu Le, Dr. Swapnil Sonkusare, Dr. Mike Koval, and Dr. Bimal Desai) thank you for challenging me, facilitating great scientific conversations and most of all, patience.

A special thank you to my work wives, Miranda, Becky, Nenja and Daniela. You ladies are the best, full stop. I'm looking forward to our super collaborations someday! To all of my current and former co-workers in the lab: you guys are awesome. Thank you for all the inside jokes, assistance, and creating a fun atmosphere along the way. A special thank you to Rob Keller for always making me laugh.

Lastly, a huge thank you to all of my Charlottesville friends for getting me out of the lab and into adventures. I have treasured our time together so much. 
CHAPTER 1. GENERAL INTRODUCTION 


\subsection{Resistance arteries in blood pressure regulation}

Mean arterial pressure is the product of cardiac output and systemic vascular resistance. Thus, the amount of resistance provided by the lumen diameter of small arteries in the periphery can have a large influence on whole-body pressure homeostasis. Form dictates function throughout the arterial tree. Large conduit arteries such as the aorta and carotid transport blood from the heart to the peripheral tissues with minimal changes in diameter. This results in minor impacts on blood pressure regulation, particularly in young adults. However, as arteries branch off the large conduits and become smaller in diameter, they do not exhibit the same structure. Multiple layers of elastin and smooth muscle are present in aorta and carotid, which are a hindrance to diffusion of substances between the SMC and EC. Smaller diameter vessels have less elastin and as they decrease in diameter, fewer layers of smooth muscle. As the arteries decrease in lumen diameter, they also form more myoendothelial junctions (MEJ), which are the hallmarks of resistance arteries. ${ }^{1-3}$ The endothelial cell (EC) or the smooth muscle cell (SMC) can send cellular projections through fenestrations in the internal elastic lamina (IEL) extracellular matrix that separates the two layers of cells. Importantly, this allows for bi-directional signaling between SMC and EC that facilitates vasoconstriction and dilation. Sandow et al has extensively characterized the literature involving the cellular source of the projection (EC, SMC, both) ${ }^{2}$ and shown using electron microscopy that large arteries such as the femoral $(\sim 900 \mu \mathrm{m}$ lumen diameter) do not form MEJ, despite the presence of IEL fenestrae. ${ }^{4}$

Poiseuille's Law indicates the radius of the tube greatly influences the resistance to flow. Thus, small changes in lumen diameter of these arteries can impact blood pressure. 
The MEJ is strategically localized in the nexus between SMC and EC to mediate and propagate signals to control blood pressure.

\subsection{The myoendothelial junction}

The MEJ was first described using electron microscopy of small arteries and capillaries in various mammalian organs. ${ }^{5}$ Because the IEL separates the EC and SMC, this physical barrier will limit small molecule transfer. Initial observations hypothesized that formation of the MEJ in small diameter arteries was a way to circumvent the diffusion barrier formed by the IEL and facilitate the transfer of nutrients from the lumen of the artery to the SMC. ${ }^{6}$ Formation of the MEJ is regulated by the presence of plasminogen activator inhibitor-1 (PAI-1), a serine protease inhibitor; mice lacking PAI-1 have fewer MEJ in coronary, cremaster and mesenteric arteries. ${ }^{7}$ Furthermore, transplantation of a PAI-1 knockout mouse's heart into a wildtype C57B1/6 mouse facilitated normal MEJ development in the coronary arteries, suggesting that circulating PAI-1 is sufficient to induce MEJ formation. ${ }^{7}$ Investigation of MEJ formation under pathological conditions has been limited, but high fat diet significantly increased the number of MEJ in coronary arteries. Whether this is a compensatory response to impairments in endothelial function is not clear.

Development and improvement of experimental techniques and creation of an in vitro model of the MEJ (Chapter 4) has further advanced the field. Sixty years later, the MEJ is appreciated as a unique signaling microdomain in the vascular wall, with expression of a multitude of proteins including enzymes, receptors and channels that all play a role in various key signaling cascades. Remarkably, many of the MEJ-localized proteins can be activated by calcium (calcium-activated potassium channels ${ }^{8,9}$, endothelial nitric oxide 
synthase $\mathrm{e}^{10,11}$, protein kinase $\mathrm{C}^{10,12}$, calcium-calmodulin kinase $\mathrm{II}^{13}$, inositol triphosphate kinase A), release calcium from the endoplasmic reticulum (inositol triphosphate receptor ${ }^{13,14}$ ), enable calcium transit into the ER or MEJ (SERCA2 ${ }^{15}$, TRPV4 channels ${ }^{16}$, connexins $^{11,17,18}$ ), bind calcium (calnexin ${ }^{18,19}$, calreticulin) or interact with proteins that mediate MEJ calcium signals (AKAP150 ${ }^{12}$, alpha hemoglobin ${ }^{20}$ ). The presence of these mediators of calcium signaling underscore the prominence of the MEJ in calcium signaling.

\subsection{Calcium signaling in the MEJ}

Calcium plays a key role in the regulation of blood pressure via a number of signaling processes in the EC and SMC. Cytosolic increases are tightly regulated by compartmentalization of calcium outside the cell via the plasma membrane and within the endoplasmic reticulum of the cell. Thus, receptor activation and cation channels can more selectively facilitate the release or influx into the cell ("calcium events") in order to exert a specific physiological effect. The development and refinement of calcium indicators has provided the ability to view these calcium events in real time and in response to agonists, antagonists and even changes in flow and pressure to an intact artery. ${ }^{9}$ Genetically coded indicators have also provided a way to specifically view calcium signaling in one particular cell type. ${ }^{21}$ Regardless of the indicator, focusing on the IEL holes provides a way to visualize calcium signals transferred between the SMC and EC and can be done in an en face preparation or in an intact pressurized artery. Of note, IEL holes are not an absolute surrogate for $\mathrm{MEJ}^{22}$, but they are the only places where MEJ can form. Because MEJ are only truly visible using electron microscopy, IEL holes are a more accessible way to visualize proteins and signaling occurring in a live artery using confocal microscopy. 
Twenty years ago, the first study to show that a calcium signal can originate in the SMC and propagate a secondary calcium response in the EC was published. ${ }^{23}$ Importantly, the EC cytosolic calcium elevation induced a vasoactive response by generating nitric oxide. Hamster cheek pouch arterioles were incubated with phenylephrine (PE) or potassium chloride $(\mathrm{KCl})$ in order to increase SMC cytosolic calcium. Calcium events were visualized via fluo-3 in the EC after application of PE. When vessels were denuded, these EC localized responses disappeared, underscoring the necessity of the EC in this response. Furthermore, phenylephrine application does not stimulate EC or have the ability to induce vasodilation. ${ }^{24-26}$ The in vitro model of the MEJ (VCCC) showed significantly less EC calcium in response to PE when MEJ formation was blocked via collagen application to the filter or gap junction signaling was inhibited. Addition of recombinant PAI-1 facilitated significantly faster calcium increases in the EC compared to the inhibition of PAI-1; PAI1 application did not alter the maximum amount of calcium. ${ }^{7}$ Because PAI- 1 enables MEJ formation, it is also necessary for heterocellular communication in response to PE. The specific molecule that is traveling between cells in this heterocellular signaling process is still debated; it is likely calcium and/or inositol trisphosphate (IP 3$)$.

Both calcium and $\mathrm{IP}_{3}$ are required for activation of the $\mathrm{IP}_{3}$ receptor (IP3R). ${ }^{27}$ In hamster feed arterioles, the localization of IP3R1 was evaluated using immuno-transverse electron microscopy. The receptor is highly localized near the plasma membrane of the EC monolayer and throughout the MEJ. ${ }^{26}$ Similar results have been shown in the in vitro MEJ mode ${ }^{14}$, indicating the machinery to facilitate $\mathrm{IP}_{3}$ induced calcium release is possible at the MEJ. Uncaging of $\mathrm{IP}_{3}$ increased EC transient receptor potential vanilloid 4 (TRPV4) 
channels mediated sparklet activity after ER calcium depletion, suggesting that $\mathrm{IP}_{3}$ can directly activate these channels at the MEJ to induce calcium influx. ${ }^{28}$

To dissect out the mechanisms of $\mathrm{IP}_{3}$ signaling to the $\mathrm{MEJ}$ in vitro, $\mathrm{EC}$ were loaded with fluo4-AM to visualize cytosolic calcium and then SMC were stimulated with PE. ${ }^{14}$ The EC based calcium events disappeared when IP3R1 expression was knocked down. Endothelial calcium responses still occurred when SMC were loaded with BAPTA to chelate cytosolic calcium. This is evidence for $\mathrm{IP}_{3}$ induced increases in $\mathrm{EC}$ calcium in response to $\mathrm{PE}$. It is apparent that altering connexin $(\mathrm{Cx})$ composition at the MEJ did not influence the transfer of the second messengers. Small interfering RNA (siRNA) targeted against Cx43 and cells from Cx40 knockouts were used; knockdown of these Cxs did not have any impairment in calcium signals transmitting from SMC to EC. However, 5phosphatase, an enzyme responsible for degrading $\mathrm{IP}_{3}$, was found to be slightly higher on the SMC side of the co-culture MEJ.

This may correlate with a recent report indicating $\mathrm{IP}_{3}$ may have a slower diffusion and shorter range of action than initially believed, due to "silencing" IP3R that bind $\mathrm{IP}_{3}$ without inducing calcium release in order to silence the $\mathrm{IP}_{3}$ signals. ${ }^{29}$ Evidence in the arteriole wall shows that the anionic carboxyfluoroscein dye did not easily transfer between SMC and EC. ${ }^{30}$ This suggests against the anionic $\mathrm{IP}_{3}$ as the diffusible factor and mediator of EC signals after PE because it is negatively charged and has a molecular mass of 420 grams per mol, making it 10 times the size of calcium. However, the diffusion range that $\mathrm{IP}_{3}$ can travel is posited to be much further than that of calcium. ${ }^{31}$

The phenylephrine response mimics prolonged sympathetic nerve stimulation. Thus, the effect on the MEJ calcium response has also been investigated with nerve 
stimulation. Norepinephrine, the physiological agonist of the alpha adrenergic receptor, can be released from sympathetic nerve terminals onto closely apposed vascular smooth muscle cells in small diameter arterioles. Nausch et $\mathrm{al}^{25}$ showed that direct electrode stimulation of the nerves innervating mouse mesenteric arteries causes an increase in IP3R calcium event ("pulsars") frequency by recruiting new sites of calcium release at the MEJ. This is accomplished by a calcium and/or $\mathrm{IP}_{3}$ generation in the SMC that is transmitted to the EC to induce calcium events via IP3R calcium release at the MEJ. The MEJ calcium elevation subsequently activated intermediate conductance calcium activated potassium channels (IK channels) without involvement of other endothelial-derived methods of vasodilation to cause negative feedback in response to the initial constriction evoked by the nerve release of norepinephrine. Thus, the MEJ in small arteries has a physiological function in opposition of vasoconstriction induced by the SMC that is not simply an artifact of PE stimulation.

Calcium events at the MEJ can also occur via EC stimulation of the muscarinic receptor. ${ }^{16,19}$ The muscarinic M3 receptor is present on $\mathrm{EC}$ and is $\mathrm{G}_{\mathrm{q}}$ coupled, increasing ER calcium release and $\mathrm{IP}_{3}$ generation. Acetylcholine or carbachol, agonists of the M3 receptor, have been shown to activate TRPV4 channels localized to the MEJ that cause calcium influx known as "sparklets". ${ }^{12,16}$ An enrichment of TRPV4 channels at the MEJ is not necessary as the presence of just a couple of channels is sufficient to evoke vasodilation. The activation is mediated by phospholipase $\mathrm{C}$, diacylglycerol (shown to be significantly enriched at the MEJ in vitro ${ }^{10}$ ) protein kinase $\mathrm{C}(\mathrm{PKC})$ and the anchoring protein that localizes PKC to the MEJ, AKAP150. ${ }^{12}$ The calcium events induced by TRPV4 are sufficient to activate IK channels downstream, further contributing to vasodilation. This 
finding expanded on the knowledge of acetylcholine simply facilitating ER calcium release in the EC to cause dilation; it showed another source of calcium, from the extracellular compartment, could enter the MEJ in a regulated manner to mediate vasodilation. In a similar calcium imaging preparation, acetylcholine was shown to increase the number of IP3R-mediated calcium events at the MEJ. ${ }^{19}$

Interestingly, there are spontaneous calcium events that occur in the EC and have been shown to specifically occur at the holes in the IEL in pressurized rat mesenteric and cremaster arterioles. ${ }^{9}$ The frequency of spontaneous events is not dependent on the hole size ${ }^{19}$, but are dependent on the IP3R. Spontaneous calcium events mediated by IP3R also occur throughout the EC of swine coronary arteries ${ }^{32}$, indicating that these physiological events are likely relevant in human arteries as well. Low arterial pressure (between 5 and $50 \mathrm{mmHg}$ ) but not pressures of $70-80 \mathrm{mmHg}$ has been shown to increase these spontaneous calcium events in rat cremaster EC from an average of 2 per minute to 5. Simultaneously, SMC calcium events are decreased from 18 per minute to $8 .{ }^{9}$ The calcium events that occur are very localized and $75 \%$ of the events do not spread outside the MEJ. These calcium events were significantly increased with TRPV4 activation and decreased with inhibition of TRPV4 channels or removal of calcium in the superfusate. ${ }^{9}$ This data provides a mechanism by which low pressure activates calcium signals at the MEJ that reduce myogenic tone.

Although functionally distinct from the peripheral circulation, the vasculature in the brain also exhibits localized calcium signals. In cerebral arterioles, TRPA1 channels can cause calcium events that occur in EC and around the holes of the IEL. ${ }^{33}$ These signals are likely not contributing to basal calcium events as the inhibition of TRPA1 had no effect 
on spontaneous events at baseline. However, addition of the TRPA1 agonist AITC increased the activity of the channels and was correlated with the calcium events seen at the MEJ. The functional consequence of increased calcium influx via TRPA1 allows the EC to oppose SMC calcium waves that promote vasoconstriction, which is similar to what occurs in the peripheral circulation.

As alluded to earlier, elevated cytosolic calcium in the MEJ can cause hyperpolarization of the $\mathrm{EC}^{24}$ that is subsequently transmitted to the SMC. Endothelial derived hyperpolarization promotes vasodilation in resistance arteries. In contrast to the discovery of nitric oxide, it is now accepted that the hyperpolarization is not likely due to just one factor. ${ }^{34}$ In the context of calcium signaling at the MEJ, activation of calciumactivated potassium channels ${ }^{35}$ causes $\mathrm{K}+$ ions to leave the EC. These channels are only expressed in the EC, with the IK channel more specifically present at the MEJ. The close proximity of the IK channel to the SMC facilitates its important function, as large diameter arteries lack this EDH response likely because of diminished MEJ formation. ${ }^{4}$

In hypertension, the localization and function of channels present at the MEJ is detrimentally altered. In stroke prone spontaneously hypertensive rats, TRPV4 and SK channel expression in the EC was significantly reduced compared to its normotensive control. ${ }^{36}$ However, acetylcholine induced vasodilation was not different. The mechanism for this is still unclear, but it may be due to compensatory ER calcium release in the rats' arterioles. Induction of hypertension using angiotensin II infusion disrupts the localization of AKAP150 protein that anchors protein kinase $\mathrm{C}$ within the holes of the IEL, which is the mechanism for decreased TRPV4 signaling in this model. ${ }^{12}$ Diet induced obesity also has detrimental effects on calcium signaling at the MEJ. After 8-10 weeks of high fat diet, 
C57B1/6 mice have reduced TRPV4 sparklets in response to CCh and direct TRPV4 activation. This may be due to significantly fewer MEJ with AKAP150 signal. ${ }^{37}$

It is evident that protein localization and calcium signaling at the MEJ is imperative to maintain physiological vascular signaling and tone. Using the VCCC to create in vitro MEJ, Straub et al looked for soluble proteins that were highly localized to the MEJ versus the rest of the EC. ${ }^{38}$ Calreticulin was one of the most enriched proteins in the MEJ, suggesting a role for Calr in mediating heterocellular signaling.

\subsection{Calreticulin structure}

Calreticulin was first discovered in 1974 in rabbit skeletal muscle and termed high affinity calcium binding protein. ${ }^{39}$ It acquired its name when it was cloned as a calcium binding protein found in the ER of non-excitable cells, deriving its name cal (calcium) + reticulin (endoplasmic reticulum) from this function. ${ }^{40}$ The Calr protein is highly conserved across mammals, invertebrates and plants, exhibiting over $70 \%$ sequence homology between mice and humans. ${ }^{41}$ It has three domains termed N, P, and C. The C domain is most important for calcium binding as it contains low affinity, high capacity binding sites for calcium along with a -KDEL amino acid sequence that promotes retention in the ER. The $\mathrm{N}$ domain facilitates the chaperone function of Calr, as it is a key mediator in protein folding within the ER. Located between the $\mathrm{N}$ and $\mathrm{C}$ domains is the proline rich domain, that has a low capacity, high affinity calcium binding domain. Expression of mutated Calr with only $\mathrm{N}+\mathrm{P}$ domains does not restore ER calcium levels in Calr deficient cells, underscoring the key role that the Calr C domain plays in ER calcium storage. ${ }^{42}$

The Calr gene is located on chromosome 8 in mice and 19 in humans. There is only one protein formed from the Calr gene, but there is a Calr fragment known as vasostatin, 
which is essentially the $\mathrm{N}$-terminal protein consisting of 180 amino acids. ${ }^{43}$ Vasostatin has interesting functions, particularly in the context of regulating EC signaling. Pike et al showed delivery of vasostatin to tumors in athymic mice significantly reduced angiogenesis by decreased EC proliferation, indicating vasostatin has a protective role against tumor growth and metastasis. ${ }^{43}$ It is still unclear if vasostatin can affect calcium signaling in the EC and why it is necessary to have this Calr fragment in the cell.

Current research on the role of Calr has intensely focused on the finding that two independent groups discovered in 2013; a somatic mutation of the Calr gene in humans is associated with essential thrombocythemia and primary myelofibrosis, cancers characterized by increased proliferation of megakaryocytes. ${ }^{44,} 45$ The mutations are primarily either a 52 basepair insertion or a 5 basepair deletion. Both lead to frameshifts in exon 9, the $\mathrm{C}$ terminal end of the protein. The $\mathrm{C}$ terminus of Calr has negatively charged amino acids, but the mutations replace these with positively charged or neutral amino acids. ${ }^{46}$ The ER retention sequence is also lost, which could cause relocation of Calr in the mutated cells.

\subsection{Calreticulin localization and function}

Although Calr was initially described as an ER localized calcium binding protein, the localization and function of Calr has continued to expand and evolve. It is well accepted that Calr has been described in multiple cellular locations outside the ER, including in the cytosol, the cell surface and the extracellular matrix. ${ }^{47}$ The existence of a protein with the -KDEL amino acid sequence outside the ER was initially confusing, as this sequence is thought to specifically keep proteins sequestered inside the lumen of the 
ER. However, it is possible that the sequence may be cleaved off, allowing for Calr exit from the ER. ${ }^{48}$

Due to the heterogeneity of Calr expression between different cell types and in response to various stimuli, Calr is a highly multifunctional protein. Physiological effects of Calr beyond calcium buffering in the ER include mediation of cellular adhesion ${ }^{49-51}$, organ development ${ }^{52,53}$, fertility ${ }^{54,55}$, gene expression ${ }^{56,57}$, mRNA binding ${ }^{58}$, and within the ER it has a lectin function and chaperone activity for both glycosylated and nonglycosylated proteins. ${ }^{59}$ The function of Calr as an ER calcium sequestering protein and ER chaperone is also highly conserved, as C. elegans Calr exhibits similar functions, despite lack of a heart and brain. ${ }^{54}$ In C. elegans and mammalian cells, expression of Calr is upregulated during cellular stress, which could be related to both calcium and chaperone functions. The importance of Calr in proper organism development and function was underscored by the finding that Calr -/- mice died in utero due to defects in the cardiac ventricle wall formation. ${ }^{52}$ However, Calr deficient cells isolated from the embryonic mice can survive, which has greatly advanced the understanding of Calr in cellular biology and signaling.

Calr deficient mouse embryonic fibroblasts show no differences in free ER calcium using a cameleon indicator targeted to the ER. ${ }^{42}$ Resting cytosolic calcium was increased in these same cells, which exhibited a blunted cytosolic calcium response to bradykinin stimulation. This was proposed to be due to impaired folding of the bradykinin receptor's ligand binding domain. Thus, the decreased $\mathrm{IP}_{3}$ mediated calcium release was likely due to the loss of the Calr chaperone function. Conversely, overexpression of Calr has been shown to increase ER calcium content. ${ }^{60,61}$ In vivo, targeted overexpression of Calr in the 
heart causes dilated cardiomyopathy which leads to subsequent heart failure. ${ }^{62,}{ }^{63}$ Calr overexpression in Madin-Darby Canine Kidney cells also caused increased cell adhesion via upregulation of integrins. ${ }^{64} \mathrm{Via}$ these studies and others, it appears that Calr expression and calcium signaling are intertwined, although the physiological relevance of this has not been clarified.

Examination of Calr homologues involve comparisons to calnexin, which shares similar functions within the ER but does not mediate cell adhesion. Both Calr and calnexin can buffer ER calcium, modulate SERCA2b activity via its N-terminal domain ${ }^{65,66}$ and promote proper folding of newly synthesized proteins. Unlike Calr, calnexin is inserted in the ER membrane and despite the similarities in function, calnexin does not completely compensate for Calr deletion. Knee et al found that Calr deficient cells exhibit activation of the unfolded protein response despite significantly upregulating Calnexin and BiP expression. ${ }^{67}$ We show in Chapter 6 that Calr and calnexin have different expression patterns in resistance arteries. The specific localization of the multifunctional Calr in the MEJ coupled with the physiological relevance of calcium signals at the MEJ suggests that Calr, but not calnexin is a mediator of heterocellular communication.

Exploration of the MEJ as a key signaling microdomain in resistance arteries is presented here. Despite the important role of lipids in calcium signaling and maintenance of cellular structure, no one has determined the lipid composition of this membrane until now. We show that in two specific instances, a protein is expressed in the MEJ and the EC monolayer, but exhibits divergent functions at the MEJ. Data in Chapter 5 and 6 continues to confirm the specificity of PE on activation of MEJ calcium signaling pathways. A combination of in vitro, ex vivo and in vivo techniques are used, in combination with 
pharmacological and genetic approaches. This allows for better understanding of the signaling machinery and mechanics within the MEJ and how they affect tissue and whole organism homeostasis. 
CHAPTER 2. THE ENDOPLASMIC RETICULUM AS A MEDIATOR OF CELLULAR SIGNALING 


\subsection{Abstract}

The ER is a prime mediator of cellular signaling due to its functions as an internal cellular store for calcium, as well as a site for synthesis of proteins and lipids. Its peripheral network of sheets and tubules facilitates calcium and lipid signaling, especially in areas of the cell that are more distant to the main cytoplasmic network. Specific membrane proteins shape the peripheral ER architecture and influence the network stability to project into restricted spaces. The signaling microdomains are anatomically separate from the cytoplasm as a whole and exhibit localized protein, ion channel and cytoskeletal element expression. Signaling can also occur between the ER and other organelles, such as the Golgi or mitochondria. Lipids made in the ER membrane can be sent to the Golgi via specialized transfer proteins and specific phospholipid synthases are enriched at ER-mitochondria junctions to more efficiently expedite phospholipid transfer. As a hub for protein and lipid synthesis, a store for intracellular calcium and a mediator of cellular stress, the ER is an important cellular organelle. Its ability to organize into tubules and project into restricted spaces allows for discrete and temporal signaling, which is important for cellular physiology and organism homoeostasis. 


\subsection{Introduction}

The ER is the largest organelle in most cells and is present in all cells but mature erythrocytes. For over five decades, it has been recognized as a key source for internal sequestration of calcium and protein translation. Although the lumen and calcium store within the ER are essentially continuous ${ }^{68,69}$, the ER is divided into distinct cellular regions: rough ER (rER, characterized by ribosomes on the surface), smooth ER (sER), and a third region, comprised in part by the nuclear membrane.

The ER consists of a membranous network of tubules and sheets extending throughout the cytoplasm (Figure 1A) with the extent of the organelle dependent on the cell's function. For example, secretory cells like pancreatic acinar cells have large amounts of rER and little sER ${ }^{70}$. Furthermore, the ER in a specific cell type can remodel based on changing cellular needs. When B-lymphocytes detect an antigen and transition to become plasma cells and secrete antibodies, their rough ER network is greatly upregulated, as measured by increases in ER resident proteins ${ }^{71}$. Interestingly, this large peripheral network exhibits independent, dynamic changes in tubule formation and degradation as part of its normal physiology ${ }^{72}$. The specialized design of the peripheral ER in this manner (Figure 1A) allows for localized signaling within specific, restricted areas of the cell. There are a number of polarized or localized molecules in the ER [(i.e., calcium related proteins (Figure 1B, C), lipid synthases (Figure 1D)] that have integral roles in signal transduction to and from the organelle. Specific localization of these molecules maintains normal cellular homeostasis, primarily by influencing lipid synthesis, trafficking and imparting strict spatial and temporal control over intracellular calcium. The close apposition of ER to other cellular organelles, including mitochondria and the Golgi apparatus (Figure 1D), 
promotes lipid and ion flux between cellular compartments. In addition to its essential roles in lipid and calcium homeostasis, peripheral ER networks may provide convenience in localized protein translation. Based on these observations, the ER is central to cellular signaling microdomains.

This review discusses the processes and molecules that comprise ER-mediated signaling and highlights those that occur in restricted spaces of the cell. For our purposes, we have defined a restricted cellular space as an anatomical area of the cell that is distinctly separate from the cytoplasm of the rest of the cell, usually encompassing a small projection of a cell or nanometer domains between organelle membranes. These cellular microdomains are identified by expression of specific proteins, receptors and/or ion channels ${ }^{73-75}$. Numerous examples can be found throughout an organism; we will focus on dendritic spines of neurons ${ }^{76}$, plasma membrane-sarcoplasmic reticulum (SR) junctions in myocytes ${ }^{77}$, and myoendothelial junctions in endothelial cells ${ }^{73}$. The ER is further able to form unique signaling microdomains in close contact with Golgi, mitochondrial, and plasma membranes, which are integral to calcium and lipid homeostasis (Figure 1C, D).

\subsection{Regulation of ER function and morphology}

Physiology dictates that structure facilitates function. An understanding of ER tubules and sheets, their formation and how the ER subsequently projects into restricted cellular spaces is essential to understanding signaling in localized areas. The specialized nature of peripheral ER structural organization defies standard energetically favorable organelle formation ${ }^{78,79}$ (e.g., a spheroid shape), so there must be some reasoning for the sheets and tubules to form. Some disagreement exists, but it is typically thought that the $\mathrm{rER}$ is found in sheet form while the sER is comprised mostly of tubules ${ }^{80,81}$. This may 
be due to the canonical views of each as hubs for protein manufacturing and calcium signaling, respectively. Moreover, it is likely that the functions of the ER are segregated based on the presence or absence of ribosomes, as the highly curved tubules may not be able to accommodate ribosomal complexes. Based on the preponderance of ribosomes and their central function in protein translation, the primary function of rER has been ascribed to protein synthesis. Conversely, peripheral ER tubules, characterized as sER, primarily control calcium signaling, manufacturing of lipids and often reside in close proximity to other organelles, thus promoting interorganelle signaling ${ }^{82}$.

The ability of ER to signal in restricted cellular spaces is dependent on the structural flexibility of the ER membrane. For a theoretical model of how this occurs, see ${ }^{83}$.] In peripheral ER tubules, there is enriched expression of reticulons (Figure 1A) and receptor expression enhancing proteins (also referred to as DP1 and REEPs), ER membrane proteins

${ }^{84}$ that interact to promote ER membrane curvature and tubule formation ${ }^{78,85}$. The caveolin proteins that form curved caveolae signaling domains in some plasma membranes are analogous in function. Mechanistically, the curvature occurs due to the proteins' two hydrophobic transmembrane segments of $\sim 30$ amino acids that insert into the outer leaflet of the ER membrane to form "hairpin"-like segments ${ }^{78,81}$. The hairpin segments do not make up the entirety of the tubule, but are stable and oligomerized ${ }^{86}$ in a scaffold or arclike fashion, thereby only required to make up 10 percent of the tubule membrane surface 82. Without reticulons, cells express ER predominantly in the form of sheets; overexpression of reticulons leads to more cells with tubular ER ${ }^{78}$. The hairpin domains of reticulons and REEPs are highly conserved across species ${ }^{82}$, further underscoring the 
importance of ER tubule formation. Once formed, these tubules can fuse to make threeway junctions with each other ${ }^{87}$ that expand the ER network.

Junctions are important for movement or equilibration of ions and other molecules with the entirety of the cellular ER network. Tubular fusion to create the junctions is mediated by a group of proteins known as atlastins, which also interact with reticulons and REEPs ${ }^{88}$. Atlastins are integral ER membrane GTPases which require GTP binding to promote tubule fusion. Overexpression or depletion of atlastins leads to long unbranched ER tubules and less three way tubular junctions, respectively ${ }^{88}$. The transmembrane protein Lunapark1 (Lnp1) is also localized to three way junctions in order to dynamically regulate the ER network. Without Lnp1 in yeast cells, the ER network collapses into a more sheet-like morphology and Lnp1 expression at the tubular ER junctions is dependent on the yeast homolog of atlastin (Sey1p) ${ }^{89}$. This translates to higher organisms as the same researchers determined that Lnp1 helps maintain ER structure in mammalian cells. Only $50 \%$ of junctions expressed Lnp1, but those that had Lnp1 were less dynamic ${ }^{90}$, indicating that junctions can form without Lnp1 but that they are less stable.

Recent work has shown ADP-ribosylation factor-like 6 interacting protein 1 (Ar16IP1), a protein that binds to atlastin, is similar to the reticulons and localizes to the ER membrane in the same hairpin fashion. This could provide another mechanism to bend the ER membrane beyond the reticulons Indeed, overexpression of Arl6IP1 led to an excess of ER tubule formation and promoted tubule stability, similar to what has been reported with reticulons ${ }^{91}$.

While there may be more molecules involved in ER structure and maintenance, Rab5, a Rab GTPase, is also essential for normal tubule formation and its deletion from $C$. 
elegans shows similar effects on ER morphology as deletion of reticulons and REEPs ${ }^{92}$. However, in contrast to the role of atlastin in binding to reticulons and REEPs, Rab5 was not shown to have direct interaction with either. Rab5 is localized on endosomal membranes, and its ability to contact the ER in this manner may account for its effects on ER formation ${ }^{92}$. This observation begins to highlight the importance of interorganelle contacts for ER distribution as well as signaling.

The cytoskeleton provides assistance with ER distribution as the microtubules of the cell facilitate localization of ER tubules in restricted spaces via several different routes. The first is the use of microtubule motors like kinesin ${ }^{93,94}$, dynein ${ }^{94}$ and myosin-Va ${ }^{95}$ that promote ER tubule movement and localization along acylated microtubules (Figure 1A). This has been shown to occur in small spaces such as neuronal axons and dendritic spines 96. Movement along established microtubules, regardless of motor presence, is termed "ER sliding" ${ }^{97}$. Other organelles such as mitochondria have been shown to utilize this same method of localization ${ }^{98}$, a possible explanation for why ER is often localized with mitochondria in the periphery of the cell. The second route relies on formation of tip attachment complexes (TAC) between the ER and the plus end of growing microtubules, which can make up approximately one fourth of the ER tubule extension that occurs in cells ${ }^{99}$. TAC relies on the direct interaction of the cytosolic $\mathrm{C}$ terminus of an integral $\mathrm{ER}$ membrane protein, stromal interaction molecule 1 (STIM1; a calcium sensor in the ER) and a protein found on the plus end of microtubules, EB $1{ }^{99}$. This was discovered via live cell imaging of the two fluorescently tagged proteins, which showed STIM1 localized with the end of the growing microtubule in a "comet-like" manner ${ }^{99}$. 
Like STIM1, the integral ER membrane protein cytoskeleton-linking membrane protein, (climp) 63, has a cytosolic domain that can bind to microtubules ${ }^{100}$ but unlike STIM1, is restricted to ER sheets. Formation of sheets is increased with expression of climp 63, which is restricted from the tubules ${ }^{81}$ and nuclear envelope due to its alpha helical segment that protrudes into the ER lumen ${ }^{101}$. When climp 63 is depleted, the morphology of the sheets is disrupted and the luminal space becomes smaller. This interaction between climp 63 and microtubules seems to be more for stabilization and holding the ER in place, rather than a method of ER movement. However, depolymerization of microtubules and actin with a variety of pharmacological compounds still results in a diffuse ER network in Xenopus egg extracts ${ }^{102}$. The functional effect of this was not fully investigated, so the resultant network may have impaired signaling. In addition to ER-microtubule interactions, receptors in the ER membrane like the IP3R have been shown to associate with actin filaments ${ }^{103}$. Actin may serve a similar function as the microtubules and helps to localize the IP3R in regions that are deficient in microtubules. Despite the evidence given above for the ER reliance on the cytoskeleton, it appears peripheral ER tubules may still form and travel without the cytoskeleton, possibly with other cellular components or signals.

The maintenance of ER structure and promotion of its localization to small spaces also relies on the lipid content of the membrane. There are only nominal concentrations of cholesterol present in the ER membrane ${ }^{104}$; the ratio of cholesterol to phospholipid composition is 0.15 compared to 1.0 in the plasma membrane ${ }^{105,106}$. When cholesterol levels increase, membrane fluidity decreases ${ }^{107}$, so this differential composition is likely advantageous for the complex folding and tubulation, plus the dynamic extension and 
retraction exhibited by the ER. An accumulation of cholesterol in the ER membranes of macrophages activates the unfolded protein response and leads to apoptosis ${ }^{108}$, supporting the evidence that amounts of cholesterol should be kept at low levels in the ER membrane. Sphingolipids are an important group of membrane lipids synthesized in the ER, but present in low quantities in the ER membrane. Their presence and packing decreases membrane fluidity ${ }^{109}$ similar to cholesterol and higher concentrations may hinder the normal formation of ER tubules and sheets.

\subsection{Lipid synthesis, trafficking and signaling centered around the ER}

The roles of cellular lipids can be grouped into several diverse functions. Lipids in the form of lipid droplets, among other functions, store energy for the organism ${ }^{110}$. Amphipathic lipids such as phospholipids are responsible for providing compartmentalization of organelles and individual cells. Additionally, bioactive lipids and regional membrane composition of lipids create membrane domains that further facilitate signaling, especially in the plasma membrane.

Lipids have a symbiotic relationship with the ER. Inositol 1,4,5 triphosphate (IP 3$)$ initiates calcium release by binding to its specific receptor on the ER membrane. Phosphatidic acid can make glycerophospholipids such as phosphatidylserine, phosphatidylcholine, phosphatidylinositol and phosphatidylethanolamine in the ER ${ }^{111}$. Cholesterol, a vital component of cell membranes, is made by the ER membrane localized HMG-CoA reductase ${ }^{112}$. The de novo pathway of sphingolipid synthesis occurs within the ER as well ${ }^{113}$. Once lipids are synthesized, the ER can export them to other organelles or the plasma membrane via a non-vesicular process. 
Lipid transfer proteins like ceramide transfer protein (CERT) are a part of the nonvesicular pathway. Ceramide is a sphingolipid, itself central to sphingolipid metabolism and synthesized in the ER. It requires transportation to the Golgi in order to be metabolized into sphingomyelin or more complex glycosphingolipids. The structure of CERT is divided into three domains: one domain targets the ER membrane with an FFAT motif ${ }^{114}$, another targets the trans Golgi membrane, and the $\mathrm{C}$ domain, containing a steroidogenic acute regulatory protein transport (StART) site ${ }^{115}$, solubilizes ceramide so that it may be transported between the organelles (Figure 1D). CERT is very specific for various chain lengths of ceramide; sphingosine and sphingomyelin are not transported despite their ability to be converted to ceramide ${ }^{116}$. In lieu of a vesicular mechanism, ceramide is taken out of the ER membrane by CERT and moved to the trans Golgi ${ }^{117}$.

The reasons why ceramide needs CERT to be trafficked this way out of the ER are not clear, but it has been hypothesized that an excess of ceramide leads to a stiffer cell membrane, thereby impeding ceramide transport in vesicles ${ }^{118}$. If ceramide traveled to the cis Golgi via vesicles, it could be glycosylated by ceramide glucosyltransferase ${ }^{118}$ into a more complex sphingolipid, thus complicating its metabolism back to sphingomyelin in the trans Golgi. By limiting the vesicular transfer and targeting the distal portion of the Golgi, CERT may be mitigating other homeostatic processes in the cell.

LY-A cells, a mutant Chinese hamster ovarian cell line, have a defect in the transfer of ceramide to the Golgi, resulting in lower sphingomyelin levels that cannot be explained by differences in synthesis or sphingomyelin precursor levels. This was determined to be due to a mutation in the LY-A CERT protein ${ }^{119}$. Additionally, ceramide is not the only lipid that utilizes non-vesicular mechanisms to leave the ER. Phosphatidylinositol and 
phosphatidylcholine are chaperoned by phosphatidylinositol transfer proteins (PITPs) which relocate them to the PM, or any other membrane deficient in either lipid species ${ }^{120}$. Sterol regulatory element binding proteins (SREBP) and cholesterol synthesis

Sterol regulatory element-binding proteins are transcription factors that are the gatekeepers for activation of genes responsible for cholesterol and fatty acid synthesis. In order to maintain lipid homeostasis, SREBPs are inhibited by high levels of sterols. The membrane sterols change the protein conformation of a SREBP-associated molecule, the SREBP cleavage activating protein (SCAP) ${ }^{121}$. When more sterol synthesis is required, SCAP becomes a chaperone for SREBP to the Golgi apparatus and the two are "sorted" into budding COPII vesicles (characterized by the expression of vesicular stomatitis virus glycoprotein). In order to leave the ER, SCAP complexes with the vesicular COPII proteins [for example, Sec23/24 and potentially Sar1B, which is known to mediate export of SREBP-2 from the ER ${ }^{122}$ ]. Increased sterols physically inhibit the interaction to prevent Golgi transport and continued sterol synthesis ${ }^{123}$. There seems to be a number of ways to regulate this signaling, as the ER proteins Insig-1 and 2 negatively regulate the SCAP/SREBP complex by binding to SCAP and keeping the complex retained in the ER membrane under the presence of high cholesterol levels in the cell ${ }^{124,}$ 125. Furthermore, the flavonoid known as xanthohumol can inhibit SREBP by preventing SCAP binding to the Sec23/24 COPII proteins and decrease expression of genes that promote cholesterol synthesis ${ }^{126}$. Importantly, these molecular events can have whole animal physiological consequences. Mice fed a high fat diet supplemented with xanthohumol were significantly less obese and had less fat accumulation in their livers ${ }^{126}$. 
Once SCAP and SREBP are transferred to the Golgi, they encounter Site 1 and 2 serine proteases S1P and S2P anchored in the Golgi apparatus ${ }^{127-129}$. Their active sites are within the Golgi lumen, where they cleave the nSREBP domain from its precursor. Once the nSREBP is cleaved, it travels to the nucleus to activate transcription of lipogenic genes. During mitosis, the spatial separation between ER and the Golgi prevents SREBP from becoming activated and turning off lipogenic gene transcription ${ }^{129}$. SREBPs upregulate the synthesis of HMG-CoA reductase, another ER membrane resident protein and the ratelimiting enzyme for cholesterol synthesis. Thus, the control of lipid synthesis and proper transfer or trafficking outside the ER can occur in a restricted manner within the cell and is important for overall cellular homeostasis.

\section{Molecules responsible for ER calcium signaling in cellular microdomains}

A central tenet of the restricted space definition is the localized expression and function of specific receptors, ion channels, ion pumps and proteins that occurs in the ER and on the ER membrane. The IP3R is one of these molecules important for ER signaling modulation, as it is a tetrameric calcium channel with six transmembrane domains present in the ER membrane. Its ligand, $\mathrm{IP}_{3}$, is a well-characterized second messenger formed after the hydrolysis of phosphatidylinositol 4,5-bisphosphate by phospholipase C. In one important study, vesicles with IP3R were incorporated into planar lipid bilayers and membrane potential was recorded after addition of calcium and $\mathrm{IP}_{3}$. This data confirmed the IP3R is only activated upon addition of $\mathrm{IP}_{3}$ and the current from the channel increases with an increase in $\left[\mathrm{IP}_{3}\right]$. Although the IP3R is dependent on $\mathrm{IP}_{3}$, the presence of calcium is needed, as $\mathrm{IP}_{3}$ cannot activate the channel alone. The activity of the channel (in the presence of $\mathrm{IP}_{3}$ ) was low at both low and high concentrations of calcium. It seems there is 
a ideal concentration of calcium $(\sim 0.25 \mathrm{uM})$ that correlates with max channel activity, represented in a bell curve manner ${ }^{130}$. In restricted spaces, this property can allow the IP3R to regulate its response more selectively to respond to physiological levels of calcium.

There are three isoforms of the $\mathrm{IP}_{3}$ receptors, known for localized ER expression but it is not known if this is a cause or result of the heterogeneity of calcium stores that has been proposed to exist in the ER ${ }^{131,132}$. There is also marked heterogeneity of expression between cell types ${ }^{133}$. Within the three isoforms, addition of molecules like ATP can enhance IP3R activity and calcium mobilization to an extent. Addition of ATP increased calcium signaling in only the first and third isoforms of IP3R, suggesting differential regulation of activity between isoforms ${ }^{134}$. The isoforms also have different sensitivities, or tendency to release calcium in response to low concentrations of $\mathrm{IP}_{3}$; IP3R2 has the highest sensitivity, followed by IP3R1 \& IP3R3. Subtype co-localization leads to an additive sensitivity to $\mathrm{IP}_{3}$ and one isoform becomes more dominant in response to ATP or calcium ${ }^{134}$. This is important for specific localization of isoforms, as they can have differential effects on the calcium signal or response to $\mathrm{IP}_{3}$ in the restricted space.

The location of the IP3R has been suggested to be mobile within the ER membrane; depending on the calcium status of the cell, the various isoforms can move within the lipid bilayer to cluster in specific areas ${ }^{132}$. Clusters of IP3R have been observed upon increases in $\left[\mathrm{IP}_{3}\right]$, relevant for the local calcium signaling in microdomains. As $\left[\mathrm{IP}_{3}\right]$ increases, the number of IP3R opening also increases, which results in sequentially larger calcium signals into the cytoplasm. However, this paradigm of IP3R mobility within the ER membrane has been questioned. Instead, clusters of IP3R may be pre-localized (rather than moving to that location upon increases in $\mathrm{IP}_{3}$ ) in order to quickly release calcium ${ }^{135}$. Ultimately, keeping 
the increases in $\mathrm{IP}_{3}$ restricted to a specific area prevents the signal from propagating throughout the cell and tissue. Cells can use inositol polyphosphate-5-phosphatase to compartmentalize calcium release by degradation of $\mathrm{IP}_{3}$ to $\mathrm{IP}_{2}{ }^{136,137}$ and the phosphatase activity depends on the amount of both $\mathrm{IP}_{3}$ and calcium ${ }^{138}$.

There is more than one way to elicit calcium release from the ER or sarcoplasmic reticulum (SR); $\mathrm{IP}_{3}$ is not the only second messenger in this physiological process. Extracellular calcium, magnesium, ATP and a number of adenine nucleotides (e.g., ADP, AMP, cAMP, adenosine) that enter the cell are able to interact with ryanodine receptors (RyR), calcium gated calcium channels on the ER and SR membrane ${ }^{139}$. RyR are the largest known ion channels and at $\sim 2 \mathrm{MDa}$, are roughly twice the size of IP3R. This extraordinary size facilitates a much higher amount of calcium released per opening ${ }^{140}$ and allows more space for interaction with regulatory and modulatory proteins. For example, RyR are part of a macromolecular complex in the SR that includes voltage gated calcium channels Cav1.1/Cav1.2, protein kinase alpha, FKBP12 and 12.6, triadin, junction, and calsequestrin ${ }^{139}$. Both the RyR and IP3R have specific calmodulin and calciumcalmodulin kinase II binding sites ${ }^{140}$, suggesting similar patterns of regulation.

Despite their large size and multitude of modulatory proteins, the RyR can be localized in myocytes to areas of the SR in close contact with the sarcolemma. The resultant localized calcium signals from RyR have been called sparks, due to their appearance with calcium indicators, and in order to distinguish them from other localized calcium signals that are caused via IP3R opening. The enrichment of RyR facilitates the localized sparks that occur in cardiomyocytes ${ }^{141}$ and vascular smooth muscle ${ }^{142}$ and can be blocked with micromolar concentrations of ryanodine. The IP3R and RyR are two of the more important 
channels regulating calcium signaling in restricted cellular spaces and have adapted to do this in a number of cell types, although the RyR is not expressed in all cell types.

\subsection{ER-mediated calcium signaling in restricted spaces}

Cytosolic concentrations of calcium are physiologically kept at nanomolar levels, while the total ER calcium concentration throughout a cell can range from 100-800 $\mu \mathrm{M}$. The ER can create localized calcium microdomains into a specific area of the cell; typically these areas consist of an ER projection into a cellular extension or the presence of the ER membrane within tens of nanometers from the PM. The structure and specific localization of proteins facilitate the restricted calcium signaling that occurs in these small spaces and this signaling has important physiological consequences ${ }^{73}$. Elementary calcium release events occur in the microdomains, but can be coupled together, as in the case of cardiomyocytes, to produce a more significant effect on the cell.

\section{Structure of restricted spaces involved in calcium signaling}

There are a number of cell types that utilize restricted spaces to localize signaling and concentrate ER expression in these areas. For example, neurons in many areas of the brain are more likely to exhibit dendrites that have spines, including the hippocampus, cerebellum, and cortex. There are a number of advantages to the addition of spines to dendrites. They make more connections with other neurons, promote plasticity within the brain and prolong calcium signals ${ }^{143}$, indicating the importance of the ER that is localized to the dendritic spine ${ }^{96,144}$. They can be differently shaped depending on the head versus neck diameter of the projection (i.e. mushroom-like, branched, stubby, thin) ${ }^{76}$, which could cause differential signaling by slowing or speeding up calcium diffusion. 
This spine-like morphology is not limited to the nervous system. Endothelial cells, which are polarized like neurons with an apical and basal side, exhibit similar projections which occur primarily in the walls of small resistance arteries ${ }^{1}$. They send projections basally across the internal elastic lamina to make contact with the opposing smooth muscle cells, creating signaling domains known as myoendothelial junctions (MEJ) that have ER projections ${ }^{14,18,19,145}$. There is evidence for microtubular presence in the MEJ, important for trafficking protein-mRNA complexes ${ }^{146}$, but this may also be a mechanism of ER localization to the MEJ.

\section{Localized expression of calcium-handling proteins}

A common theme, despite differences in cell type and physiological output, is the specific localization of proteins and receptors involved in calcium signaling to the restricted spaces. This expression primarily occurs due to a protein's specific association with the ER and confers the ability to initiate or terminate calcium signaling, which is vital to segregate signaling from the cytoplasm. The IP3R is one of the best examples of proteins involved in localized ER signaling as it has been shown to be localized in nerve terminals ${ }^{147}$, dendritic spines ${ }^{96}$, at the MEJ ${ }^{14,145}$, and the nuclear envelope of cardiomyocytes ${ }^{148}$. Because the IP3R is a transmembrane ER protein, it is also strategically located to interact and influence luminal protein localization.

Chaperone proteins within the lumen of the ER or SR can be highly localized to restricted spaces to participate in calcium signaling and buffering. At triadic junctions, where localized calcium signaling occurs in myocytes, calsequestrin is enriched, along with the ER transmembrane proteins triadin and junctin, and the three molecules form a macromolecular complex with the RyR ${ }^{149}$. Calreticulin, an ER resident protein and 
calcium buffer ${ }^{150}$, is present in the MEJ ${ }^{151}$. This illustrates the capacity of calcium buffers to localize to specific parts of the ER lumen and potentially increase calcium storage within the reticular network. This in turn can modulate specific processes involved in calcium release. Ultimately, the combination of calcium release, calcium uptake, calcium sensing and calcium storage in restricted spaces is conferred by the localized expression of specific proteins.

Signaling processes: implications for function

Calcium as a signaling molecule has a broad range of actions: fertilization, proliferation, secretion, metabolism. However, in specific localized cellular regions, the effects of calcium signaling seem to be more limited to events that control contraction (cardiomyocytes, smooth muscle cell) and synaptic plasticity (neurons).

Calcium induced calcium release (CICR) is the foundation of excitationcontraction coupling and is a physiological process that is founded on signaling in a restricted space. It occurs in a gap between the SR and the plasma membrane that is roughly 12 nanometers wide. When an L-type calcium channel opens upon cellular depolarization, the extracellular calcium influx into this area activates roughly four RyR located on the SR. Using calcium imaging via a patch clamp technique combined with confocal microscopy, the opening of the calcium channel on the PM looks like a "sparklet", a very small calcium release signal, while the calcium leaving the SR appears as "sparks", a localized but more robust calcium release ${ }^{77}$. Combining the rich source of extracellular calcium with the sequestered SR source is a perfect design to spatially manage the calcium response within the cardiomyocyte, and ultimately the contractility of the heart. 
CICR is not limited to myocytes. When a hippocampal pyramidal cell is stimulated with an electrode to depolarize the membrane, ionotropic NMDA receptors are activated by glutamate and glycine, triggering an excitatory, post synaptic calcium transient that is restricted to the dendritic spine. Cyclopiazonic acid, an inhibitor of the sarcoplasmicendoplasmic reticulum ATP-ase, greatly diminished this transient, suggesting that the calcium transient needs a small calcium influx via NMDA activation to subsequently trigger calcium release from the ER ${ }^{144}$.

In a different pathway but with similar physiological effects, parallel fibers synapse on cerebellar Purkinje neurons, releasing glutamate that activates local metabotropic glutamate receptors (mGluR) located on the dendritic spines. Smooth ER localized here exhibits the presence of IP3R, which releases calcium in response to the $\mathrm{IP}_{3}$ generated via mGluR activation. The IP3R activation is confirmed as a delayed calcium response (it appeared after the CICR calcium signal) using confocal microscopy and inhibited by addition of heparin ${ }^{152}$. Spread of the signal was restricted, due to the short half-life of $\mathrm{IP}_{3}$ combined with the limited physical space. The combination of depolarization and parallel fiber signaling, with ER calcium release is a hallmark of long term depression. The importance of the depolarization/mGluR generation of $\mathrm{IP}_{3}$ and calcium release from the ER signaling cascade in long term depression and synaptic plasticity is underscored by no changes in the excitatory post synaptic current or potentials of Purkinje neurons (e.g., no long term depression) of mGluR1 knockout mice ${ }^{153}$, inhibition of IP3R with heparin ${ }^{154}$ and in cerebellar neurons of IP3R1 knockout mice ${ }^{155}$.

Thus, restricted neuronal expression of ER and calcium signaling is important for structural plasticity of the brain, which relies on long term depression. Structural plasticity 
occurs when the architecture of the brain is physically changed as a result of learning (e.g., synapses move and strengthen). In the hippocampus, this can be directly related to learning and memory, while in the cerebellum it is related to motor learning and physical coordination.

Similar to structural plasticity in dendritic spines, repeated and restricted calcium signals in the vascular wall have important physiological outcomes and facilitate cell-cell communication. The MEJ is a small projection, approximately $0.5 \mu \mathrm{m}$ wide and long, so localized calcium release from IP3R had been difficult to image for many years. Using a unique mouse model that has a calcium biosensor in the endothelium (GCaMP2) and small mesenteric resistance arteries, holes in the internal elastic lamina were, for the most part, associated with calcium pulsars, IP3R mediated calcium release event from the ER. These pulsars occur on a regular basis (about 270ms apart) and in the same locations (i.e. the holes in the IEL). In comparison to other restricted calcium signals in vascular smooth muscle, measurable pulsars are slower and have smaller amplitude. Pharmacological inhibition of mediators in the $\mathrm{IP}_{3}$-calcium release pathway showed that there is a basal level of $\mathrm{IP}_{3}$ in $\mathrm{EC}$ that can cause calcium pulsars ${ }^{19}$. Other researchers have used pressurized rat mesenteric arteries with Oregon Green 488 BAPTA-AM dye to show that the ER mediates spontaneous calcium events at the MEJ ${ }^{156}$. The exact mechanism and pathway for the ERmediated signals is still under investigation: calcium/calmodulin kinase II has been suggested as one mediator of IP3R pulsars ${ }^{145}$. ER calcium signals at the MEJ are an important way to allow communication between endothelium and smooth muscle, thus far they have only been shown to originate from the IP3R. Regardless, they are similar to the other manifestations of $\mathrm{IP}_{3}$ mediated calcium release in restricted spaces. 


\subsection{ER signaling involving cross talk with other organelles}

The ER is dispersed throughout the cell and as such makes connections with a number of other organelles ${ }^{157}$. These connections, typically separated by a $10-40 \mathrm{~nm}$ distance, are purposefully designed to take advantage of other cellular resources and are likely sER due to a lack of ribosomes ${ }^{82}$.

\section{Mitochondria (Mitochondrial Associated Membrane)}

Mitochondria are close partners to the ER and the two organelles can make contact at the mitochondrial associated membrane (MAM), which acts as a bridge between ER and mitochondria. It is estimated that 5-20 percent of the mitochondrial surface consists of MAM ${ }^{158}$ and that areas of mitochondrial division within the cell are concomitant with ER tubule presence ${ }^{159}$. Lipid and calcium transfer are the prime reasons for their association. The ER makes a number of lipid species [phosphatidylserine ${ }^{160}$, for example] that the mitochondria must import. Furthermore, increases or decreases in mitochondrial uptake of calcium via its uniporter can be a trigger for autophagy or apoptosis, respectively. The IP3R is clustered in this membrane ${ }^{161}$ and a slight increase in calcium near the mitochondria can increase mitochondrial metabolism, causing increased synthesis of ATP. Mitochondria can also buffer the calcium signal caused by ER calcium release, which can lead to an increase in IP3R activity ${ }^{161}$.

Another important function of the MAM is for phospholipid synthesis ${ }^{162}$. A number of enzymes that synthesize lipids are located in this region, one specifically being phosphatidylserine synthase ${ }^{163}$. Once phosphatidylserine is made in the MAM, it can easily be transported to the mitochondria nearby (Figure 1D). In contrast to the vesicular 
transfer of lipids described earlier, the MAM is able to transport lipids in a nonvesicular fashion, likely via movement within the membrane to the mitochondria.

Recent research has focused on proteins that play central roles in linking the membrane of the two organelles. One of them is trichoplein/mitofusin which interacts with Mitofusin 2, a GTPase localized to both the outer mitochondrial membrane and the ER, indicating its presence in the MAM ${ }^{75,164}$. In cells that lack mitofusin 2, calcium coupling between the ER and mitochondria is decreased, probably due to an increased distance between the organelles. Interestingly, these experiments indicated mitofusin 2 also regulates the structure of the ER network as the knockout cells exhibited fragmented ER 164. Presenilin proteins 1 and 2 are found at the interface of the MAM and mutations in their structure are implicated in Alzheimer's disease. The second isoform is responsible in part for tethering ER to mitochondria and interorganelle calcium transfer ${ }^{165}$, so it is evident that the proteins controlling these interactions are important for normal cellular homeostasis.

\section{Plasma Membrane}

The ER-plasma membrane junction with a separation of 7-30nm was first described in excitable cells in regards to calcium flux and these observations remain highly relevant today ${ }^{166}$. Not only is the signaling that occurs between the ER-plasma membrane localized in a small space, but the junctions themselves could be polarized to a specific region of the cell, as only $5 \%$ of the PM has these junctions ${ }^{69}$. Lipid transfer can occur between the membranes as well, but this has been shown solely in drosophila and yeast cells; its relevance to mammalian cell signaling and physiology is unclear. 
Store operated calcium entry (SOCE) is calcium signaling that occurs in the space between the ER and plasma membrane, originating from the ER (Figure 1C). It is the main method of calcium entry in non-excitable cells but it was not until the past ten years that the proteins responsible, STIM 1/2 and Orai 1-3, were understood and integrated as part of the process. When ER calcium is depleted, by any means (IP3R-mediated, experimentally with thapsigargin or otherwise), SOCE occurs and extracellular calcium enters the cell. This is reflected in the emergence of the inward calcium release activating current (ICRAC) 167. This current is due to the CRAC channel being permeable to calcium. The Orai gene encodes the CRAC channel and its proteins make up the pore of the channel that is responsible for the calcium influx. STIM1 is localized to the ER membrane as a single pass transmembrane protein, where it can make a physical interaction with the $\mathrm{N}$ and $\mathrm{C}$ termini of Orai1 ${ }^{168}$.

The mechanism by which SOCE occurs begins with the depletion of ER calcium and the ability of STIM1 to sense this deficit and oligomerize, thus setting off a signaling cascade in order to refill the ER calcium level ${ }^{169}$. Its structure contains an EF hand, a structural domain that is known to bind calcium, in its ER-lumen located N-terminus. STIM1 translocates to an area of the ER near the PM (punctates corresponding to STIM1 appear within 10-25 $\mathrm{nm}$ of the PM) to subsequently activate the CRAC current. When STIM1 is expressed alone and calcium is depleted, STIM1 punctates still appear localized near the PM. However, when GFP-tagged Orail is expressed alone and thapsigargin is added to the cell, no punctates are seen until both STIM1 and Orai1 are expressed together 168. 
The tubule design of the peripheral ER can be further appreciated in this context, as it was shown that deletion of reticulon 4 caused ER tubules to morph into sheets 78,170 and this affected the cellular SOCE but none of the other important ER functions ${ }^{170}$.

\subsection{Conclusion}

Microdomains within the cell allow for compartmentalized ER signaling that can be separate from overall cell function or be integrated into a larger physiological response. Although energetically unfavorable, the formation of the diffuse tubules of the peripheral ER network allows for localization into restricted spaces of cells, such as neuronal dendritic spines and for contact with other organelles (Figure 1). The purpose of ER-mediated signaling within a small space permits molecules with vulnerability to degradation or modification, like $\mathrm{IP}_{3}$ or ceramide, to have a greater impact. Localized ER signaling saves energy and time as proteins, lipids and ions do not have to traverse large distances, allowing a faster, more efficient effect. The extensive and unique properties of the ER allow it to localize and enhance otherwise weak signals, which can have important functional effects on physiological processes within an organism. 
FIGURE 1. ENDOPLASMIC RETICULUM LOCALIZATION AND SIGNALING IN MICRODOMAINS.

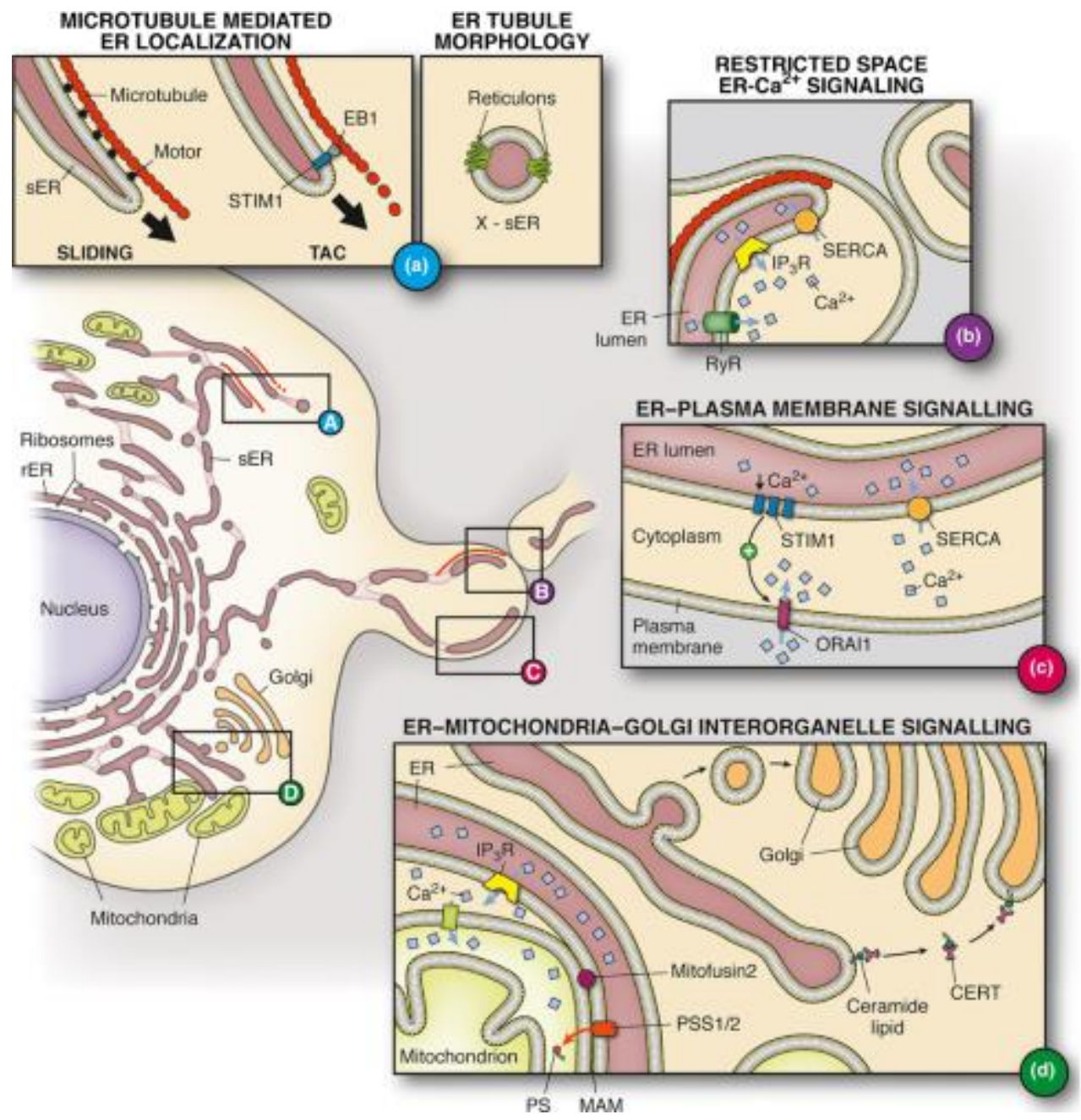


Figure 1. Endoplasmic Reticulum (ER) localization and signaling in microdomains. A, Examples of microtubule (red circle)-mediated endoplasmic reticulum (ER) trafficking, (1) ER sliding and (2) tip attachment complexes (TAC). Reticulons mediate ER tubule curvature by insertion into the membrane in a hairpin fashion. B, Spatially restricted calcium signalling that occurs from the ER. This is characterized by the expression of specific receptors $\left(\mathrm{IP}_{3} \mathrm{R}, \mathrm{RyR}\right)$ and cytoskeletal elements (microtubules). C, ER-PM signalling as illustrated by STIM1-Orai1 interactions to refill the ER. D, Interorganelle signalling occurs in a restricted space. (1) ER to Golgi transfer of lipids can occur via an unconventional, non-vesicular (ceramide transfer protein, CERT) method. (2) The mitochondria-associated membrane (MAM) is a distinct section of the ER membrane tethered to the mitochondria by proteins and is enriched with $\operatorname{IP}_{3} \mathrm{R}$ and phospholipid synthases (Phosphatidylserine synthase 1 and 2, PSS 1/2) to facilitate ER-mitochondria signalling. 
FIGURE 2. AN EXAMPLE OF ENDOPLASMIC RETICULUM IN RESTRICTED SPACES: THE MYOENDOTHELIAL JUNCTION.

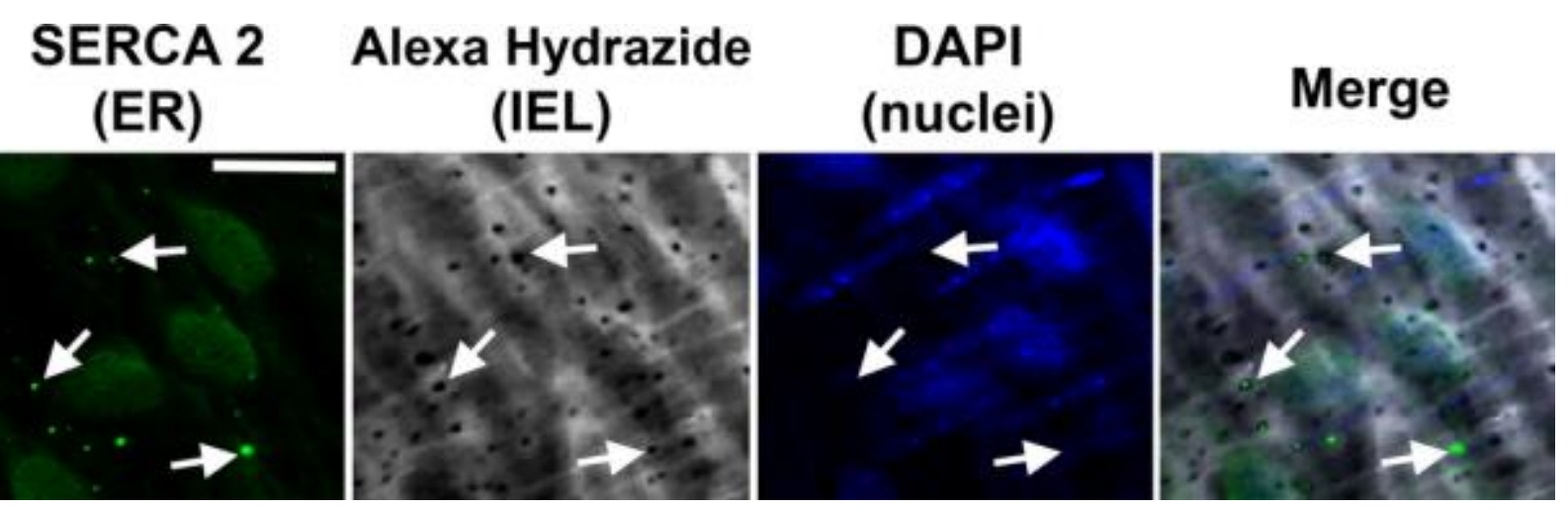

Figure 2. An example of ER in restricted spaces: the myoendothelial junction. An antibody against SERCA was used as a marker for endoplasmic reticulum (ER). In this example, thoracodorsal arteries were removed from a mouse, cut longitudinally and viewed en face. The arteries were immunolabelled for SERCA, isoform 2 (green), and counter stained with Alexa Hydrazide 633 to mark the internal elastic lamina (IEL) between endothelium and smooth muscle and reveal holes where myoendothelial junctions reside (grey). DAPI staining was used to mark endothelial and smooth muscle nuclei (blue). Arrows point to examples of ER in holes of the IEL and scale bar is $10 \mu \mathrm{m}$. 
CHAPTER 3. MATERIALS AND METHODS 


\subsection{Materials and Methods for Chapter 5}

VCCC: Pharmacological compounds were added to the media except where noted.

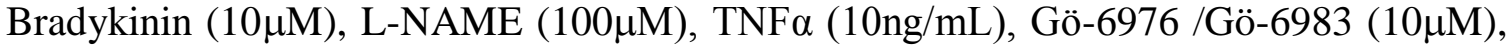
BAPTA $(20 \mu \mathrm{M})$, Thapsigargin $(2 \mu \mathrm{M})$ and Xestospongin $\mathrm{C}(20 \mu \mathrm{M})$ were all added to the EC side. PE $(50 \mu \mathrm{M})$ was added to the SMC side. The gap junction inhibitor 18-GA $(50 \mu \mathrm{M})$, the NO donor GSNO $(100 \mu \mathrm{M})$ were added to both EC and SMC.

Immunofluorescence: Transverse sections of the VCCC filter were fixed in 4\% PFA, transversely embedded in paraffin and sectioned. Slides were incubated overnight with antibodies to alpha 1 adrenergic receptor (Santa Cruz), bradykinin receptor 2 (Abcam), total eNOS, S1177 eNOS (BD Bioscience), or calreticulin (Thermo-Fisher).

Western Blotting: The VCCC fractions (EC/MEJ/SMC) were scraped in 1X PBS and placed in lysis buffer as previously described ${ }^{7,38}$. Then EC/MEJ/SMC fractions were sonicated and subjected to electrophoresis as described ${ }^{7}$. Briefly, SMC were thoroughly scraped from one side and EC were thoroughly scraped off the other side. The remaining protein and membranes within the filter pores of the transwell are considered the MEJ fraction; the whole filter was immersed in protein lysis buffer after removal of EC and SMC. Then EC/MEJ/SMC fractions were sonicated and subjected to electrophoresis as described ${ }^{7}$. Blots were probed for S1177 eNOS, stripped and reprobed for total eNOS. Band intensities were quantified using Li-Cor Odyssey Image Studio Software and values for S1177 eNOS were normalized to total eNOS.

Measurement of Glycerolipids, Phospholipid Derivatives and Sphingolipids:

VCCC fractions and the filter were harvested in 1X PBS without addition of protein lysis buffer. Phospholipid derivatives and sphingolipids were extracted and quantified by liquid 
chromatography-mass spectrometry, as previously described ${ }^{171}$ with the following modifications. Extracted glycerolipids and phospholipids were resuspended in methanol:chloroform:millipure $\mathrm{H}_{2} \mathrm{O}$ (4:1:1) and analyzed after separation with a Kinetex C18 column. Mobile phase A consisted of $60 \%$ acetonitrile, $40 \% \mathrm{H}_{2} \mathrm{O}, 0.1 \%$ formic acid and $1 \mathrm{mM}$ ammonium formate. Mobile phase B consisted of 90\% isopropanol, $10 \%$ acetonitrile, $0.1 \%$ formic acid, and $1 \mathrm{mM}$ ammonium formate. Total flow rate was $0.8 \mathrm{~mL} / \mathrm{min}$. Sphingolipid extracts were divided in half for the analysis of ceramides and sphingosine-1-phosphate (S1P) species. For ceramide measurements, $500 \mu \mathrm{L}$ of chloroform and $1 \mathrm{~mL}$ of millipure $\mathrm{H}_{2} \mathrm{O}$ were added to each sample. Extracts were vortexed and centrifuged at 2000rpm for $10 \mathrm{~min}$. The organic phase was isolated, dried down under $\mathrm{N}_{2}$ and resuspended in $97 \%$ acetonitrile, $2 \%$ methanol, $1 \%$ formic acid $(\mathrm{v} / \mathrm{v} / \mathrm{v})$ with $5 \mathrm{mM}$ ammonium formate. For S1Ps, one half of the sphingolipid extract was centrifuged at maximum speed for 10min, and the supernatant was dried down and resuspended in methanol. Both ceramides and S1Ps were analyzed by liquid chromatography-mass spectrometry following previously established methods ${ }^{172}$. Values were normalized to an internal standard and then to protein concentration. For measurement of cholesterol, total lipids were extracted from the VCCC using an adapted Folch method as previously described ${ }^{173}$. Samples were then assayed for total cholesterol using the fluorometric Amplex Red Cholesterol Assay kit (Thermo Fisher). Background fluorescence was subtracted from the initial fluorescent value.

Protein kinase $C$ assay: Each of the VCCC fractions were stimulated with PE or no treatment for 1 minute, then harvested into lysis buffer and ran in triplicate (5ug lysate/well) following the assay protocol and recommended lysis buffer (Abcam). 
Background absorbance was subtracted from the initial values, then normalized to protein concentration and presented as relative activity.

Static adhesion assay: Leukocyte adhesion to the EC of the VCCC was performed by co culturing human umbilical vein endothelial and smooth muscle cells on the opposing sidesof transwell inserts. THP1 monocytes were grown separately in RPMI media without antibiotics. Before addition to the VCCC, the monocytes were loaded with $5 \mu \mathrm{M}$ calcein AM for 20 minutes in Krebes-Hepes. Four hours before harvest, all transwells (except control) were incubated with TNF- $\alpha$ in Krebs-Hepes buffer. BK, PE and GSNO were added after four hours of TNF- $\alpha$. After 1 minute of agonist stimulation, calcein loaded THP1 monocytes were added to the EC side of the VCCC for 5 minutes. Cells were then gently washed with Krebs-Hepes and fixed with 4\%PFA for 20 minutes. The transwell filter was excised and mounted on a slide and adherent leukocytes were quantified.

Cyclic GMP assay: Cyclic GMP was determined according to kit instructions (Cell Signaling) and as previously described ${ }^{20,38}$.

Statistics: One way analysis of variance was used with Tukey post-test or Dunnett's multiple comparison post-test. 


\subsection{Materials and Methods for Chapter 6}

Animals. Male mice were 10-20 weeks of age. Vascular endothelial cadherin Cdh5$\mathrm{ER}^{\mathrm{T} 2+}$ (C57BL6/J background; kind gift from Dr. Ralf Adams, Max Planck Institute, Germany) mice were bred to calreticulin floxed mice (Calr ${ }^{\mathrm{f} / \mathrm{fl}}$; DBA/2J background, kind gift from Dr. Marek Michalak, University of Alberta, Canada). At 6 weeks of age, the mice were injected intraperitoneally with $0.1 \mathrm{~mL}$ of peanut oil (EC Calr fl/fl) or $10 \mathrm{mg} / \mathrm{mL}$ tamoxifen (Sigma; EC Calr $\Delta / \Delta$ ) for 10 consecutive days. Wild type C57Bl/6 male mice were purchased from Taconic. All mice had standard 12 hour light/dark cycles and were fed normal chow. All animals were used according to University of Virginia Animal Care and Use Committee guidelines and were euthanized with inhaled $\mathrm{CO}_{2}$.

Antibodies. Calreticulin (Thermo Fisher) was used for western blotting (1:1000), immunofluorescence (1:500), immuno-transmission electron microscopy and coimmunoprecipitation (1:50). Alexa Fluor 488 conjugated calreticulin (1:50; Abcam) and PerCP-Cy5.5 conjugated CD31 (1:50; BioLegend) were used for flow cytometry experiments. Calnexin and ERp29 (1:300; Abcam) were used for immunofluorescence. LICOR IR-DYE 680 or $800 \mathrm{CW}$ secondary antibodies $(1: 15,000)$ were used for western blotting. Alexa Fluor $568 \mathrm{f}\left(\mathrm{ab}^{\prime}\right) 2$ secondaries (1:500; Life Technologies) were used for immunofluorescence.

En face arterial immunofluorescence. Third order mesenteric arteries were dissected free of perivascular fat in Krebs-Hepes buffer, cut longitudinally and pinned open with the endothelial cell side facing up on a Sylgard block. Arteries were fixed in $4 \%$ paraformaldehyde and blocked with normal serum. Primary antibodies were incubated overnight at $4{ }^{\circ} \mathrm{C}$ with rocking. Holes in the IEL (HIEL) were counted via multiple fields 
of view and normalized to the area. For digitonin experiments, digitonin was added to blocking buffer instead of Triton-X100. For brefeldin A experiments, brefeldin A (1 $\mu \mathrm{g} / \mathrm{mL}$ ) was added for 4 hours prior to fixation. Counting of fluorescent HIEL was determined by counting holes found to have pixel intensity above threshold and scoring them against the total number of holes. Images were taken using an Olympus Fluoview 1000 upright confocal microscope and collected using Fluoview software.

Mouse genotyping and endpoint PCR. To distinguish EC Calr $\Delta / \Delta$ mice from EC Calr fl/fl mice, the following primers were used: Forward: 5'AATGACCAGAGTTGATTCCAAGG-3'; $\quad$ Reverse:5' CCAGAATGCTGATCTTCATACCATGG-3'. Automated genotyping (Transnetyx) was used to determine EC Calr wt/wt versus EC Calr fl/fl mice. Tailsnip samples were digested with proteinase K, genomic DNA was extracted using Qiagen DNeasy Blood and Tissue Kit and samples were run on a $1 \%$ agarose gel. A band of approximately $400 \mathrm{~kb}$ indicated excision of calreticulin in EC.

Expression of calreticulin in endothelium from EC Calr fl/fl and EC Calr $\Delta \Delta$. Diaphragm tissue was dissected free from intercostal muscles and the aorta, vena cava and esophagus (thus containing exclusively microvascular EC) ${ }^{174}$ from EC Calr fl/fl and EC Calr $\Delta / \Delta$ mice was dissected out, cut into small pieces and disassociated at $37^{\circ} \mathrm{C}$ for 45 minutes with collagenase XI (400U, Sigma), collagenase I (1000U; Sigma), DNAse (120U; Sigma) and hyaluronidase (250U; Sigma). Cells were strained through $100 \mu \mathrm{m}$ filters and then $40 \mu \mathrm{m}$ filters. Fixable yellow live/dead stain (1:500; Life Technologies) was added to exclude non-viable cells. Fc Block (1:00; Rat Anti-Mouse CD16/CD32, BD Biosciences) was added 10 minutes before extracellular staining with CD31 antibody (25 minutes). Cells 
were then fixed and permeabilized for 20 minutes (BD Cytofix/Cytoperm) before calreticulin antibody was added for 30 minutes. Compensation beads (UltraComp eBeads; eBioscience) were used for the single stain CD31 control to determine the gating strategy. Mean fluorescence intensity for Calr was obtained from the CD31+, CALR+ cells.

Calcium imaging. Third order mesenteric arteries from EC Calr fl/fl and EC Calr $\Delta / \Delta$ mice were dissected free of fat, cut longitudinally and pinned open, endothelial side

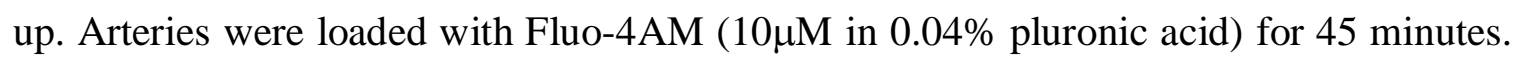
All experiments were performed in physiological salt solution and the presence of the TRPV4 inhibitor HC067047 (1 $\mu \mathrm{M}$; Tocris) and nifedipine ( $1 \mu \mathrm{M}$; Sigma) to block L-type calcium channel events in smooth muscle. Calcium events were imaged using an Andor Revolution WD (with Borealis) spinning-disk confocal imaging system (Andor Technology) comprised of an upright Nikon microscope with a 60X water dipping objective (numerical aperture 1.0) and an electron multiplying charge coupled device camera. Samples were excited at $488 \mathrm{~nm}$ with a solid-state laser and emitted fluorescence was collected using a 525/36-nm band-pass filter. The images were recorded before and 2

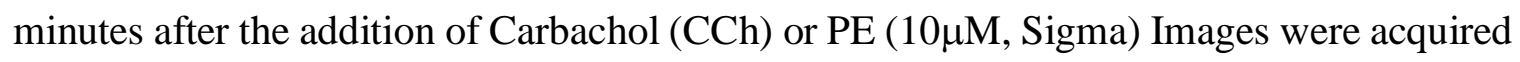
at 30 frames per second, with $512 \times 512$ pixels in the field of view. A custom designed program (Adrian Bonev, University of Vermont) was used for the image analysis. Images were filtered using a Kalman filter plugin imported from ImageJ. All the event sites within the recording duration ( 1 minute) were detected manually using the increase in fluorescence over averaged image. Fractional fluorescence traces (F/F0) were generated for each site using a 1.7 sq. micron ( $5 \times 5$ pixels) region of interest. All the events that crossed a threshold of $1.3 \mathrm{~F} / \mathrm{F}_{0}$ were included in the analysis. 
Calcium imaging in aged mice. A subset of mice was injected as normally at 6 weeks with peanut oil or tamoxifen. Then mice were allowed to age until they were at least 75 weeks old. Third order mesenteric arteries were dissected out for en face calcium imaging and subjected to the same conditions and protocol as calcium signaling in young mice. Aortas were also harvested and snap frozen for qPCR analysis.

Pressure myography. Third order mesenteric arteries were dissected free of fat, cannulated on glass pipets, perfused to remove blood and pressurized to $80 \mathrm{mmHg}$ for all experiments. All experiments were performed in Krebs-Hepes buffer at pH 7.40-42 with $2 \mathrm{mM} \mathrm{Ca}^{2+}\left(118.4 \mathrm{mM} \mathrm{NaCl}, 4.7 \mathrm{mM} \mathrm{KCl}, 1.2 \mathrm{mM} \mathrm{MgSO}_{4}, 4 \mathrm{mM} \mathrm{NaHCO}_{3}, 1.2 \mathrm{mM}\right.$ $\mathrm{KH}_{2} \mathrm{PO}_{4}, 10 \mathrm{mM}$ HEPES, $6 \mathrm{mM}$ glucose) using a Danish MyoTechnology system and software. Only vessels that developed tone and fully dilated to $1 \mu \mathrm{M}$ NS309 were used. For CCh dose response and maximum dose experiments, arteries were preconstricted with PE $(10 \mu \mathrm{M})$ before additive doses of CCh (10-9 to 10-3). Initial tone was measured at $80 \mathrm{mmHg}$ without addition of any drugs. Potassium chloride was used at $40 \mathrm{mM}$ concentration and sodium nitroprusside was used at $1 \mathrm{mM}$. Calcium free Krebs-Hepes buffer ( 1 mM EGTA and $10 \mu \mathrm{M} \mathrm{SNP})$ was added at the end of all experiments to determine maximum passive diameter. Brefeldin A vasoreactivity. Third order mesenteric arteries were dissected for vasoreactivity and placed in Krebs-HEPES buffer with $1 \mu \mathrm{M}$ Brefeldin A or vehicle (0.1\% DMSO) on ice for 1-4 hours (no significant difference was seen in response after 1 versus 4 hours). Then PE dose responses were performed as usual.

Peptide vasoreactivity. ITPKA peptide and scrambled peptides $(10 \mu \mathrm{M}$; AnaSpec, Inc.) were reconstituted in water and loaded into the artery lumen and bath superfusion. 
Arteries were allowed to equilibrate for 30 minutes in the presence of peptide before beginning the PE dose response.

Radiotelemetry blood pressure. EC Calr fl/fl at 10 weeks of age were implanted with radiotelemetry catheters via the carotid artery under inhaled isofluorane anesthesia as previously described ${ }^{20}$. After 7 days of recovery, a five day baseline was recorded. Mice were then stratified into groups for daily injection with tamoxifen (EC Calr $\Delta / \Delta$ ) or vehicle control (EC Calr fl/fl) for 10 days. Mean arterial pressure and heart rate was continuously monitored and averaged daily. Each mouse was compared back to its own 5 day preinjection baseline. Acute measurement of blood pressure: Mice were randomized to EC Calr fl/fl or EC Calr $\Delta / \Delta$ and injected at 6 weeks of age to induce knockout. Radiotelemetry catheters were implanted at 10 weeks of age and mice were allowed to recover for 1 week. Then, baseline night pressures and heart rate were averaged for 3-5 days. A 2 hour baseline was taken prior to PE injections. Mice were weighed and then injected intraperitoneally with $0.1 \mathrm{mg} / \mathrm{kg}$ PE dissolved in sterile saline. The values for the first 15 minutes after PE injection were averaged and compared to the 2 hour baseline for each individual mouse. Angiotensin II blood pressure: A separate cohort of mice were implanted with radiotelemetry catheters after peanut oil or tamoxifen injection at 6 weeks. After recovering for one week, a 4 day baseline was obtained. At day 0 , the mice were implanted with osmotic pumps containing angiotensin II (1000ng/kg/min) and pressure measurements were obtained for 15 days.

Peak calcium fluorescence. A subset of Fluo-4 arteries from the calcium experiments were incubated for 20 minutes with $0 \mathrm{mM}$ extracellular calcium and $20 \mu \mathrm{M}$ cyclopiazonic acid to inhibit refilling of endoplasmic reticulum (ER) stores. The resulting 
fluorescence was quantified in arbitrary fluorescent units and used to approximate ER calcium store content.

GCaMP-ER fluorescence following calreticulin siRNA in human EC. Primary human aortic EC (Lonza) were plated on 6 well dishes. Lipofectamine 2000 (Thermo Fisher) was used to transfect cells, first with calreticulin siRNA (5nM; Origene) and 24 hours later, with GCamp5 (pN1-GCamp-ER with backbone of pEGFP-N1 from Clontech; kind gift from Dr. Michael Kotlikoff, Cornell University) for 6 hours in 0.5\% FBS EC media (Lonza) without antibiotics. After 6 hours of transfection, media was removed and replaced with 5\% FBS EC media (without antibiotics). Knockdown of calreticulin was quantified by comparing the band for calreticulin against total protein and then plotting compared to scrambled siRNA. Samples were run on FACSCalibur machine (Becton Dickinson) and FlowJo software was used for analysis. For confocal images of GCaMPER transfected cells, EC were viewed via brightfield and GFP (488 excitation) and stimulated with $35 \mu \mathrm{M}$ ATP to deplete ER calcium. Calr siRNA timecourse experiments. The level of Calr protein was measured at 24, 48 and 72 hours after transfection with Calr siRNA $(5 \mathrm{nM}, 25 \mathrm{nM})$ or scrambled siRNA $(5 \mathrm{nM}, 25 \mathrm{nM})$. The level of Calr expression was first normalized to total protein, then compared to Calr expression after scrambled siRNA.

$\underline{\text { Western blots. }}$ To obtain total protein lysates, cells were isolated into ice cold lysis buffer (150mM NaCl, 1mM EGTA, 1mM EDTA, 10mM Tris, 0.5\% Triton X100, 0.5\%NP$40 \mathrm{pH} \mathrm{7.5)}$ and protease inhibitor cocktail and phosphatase inhibitor cocktails 2 and 3 $(10 \mu \mathrm{L}$ per $1 \mathrm{~mL}$; Sigma) were added the day of experiment. Protein concentration was determined using a bicinchoninic assay (BCA; Thermo Fisher). Gel electrophoresis was run using NuPAGE MOPS running buffer and 4-12\% Bis-Tris gels (Invitrogen) and 
transferred to $0.2 \mu \mathrm{m}$ nitrocellulose (GE Healthcare Amersham). Prior to the blocking step, total protein stain was added for 5 minutes, washed and then scanned to determine total protein concentration (ReVERT total protein stain; LI-COR). All blots were blocked with $5 \%$ bovine serum albumin in tris buffered saline $+0.1 \%$ Tween, then incubated with primary antibodies overnight at $4{ }^{\circ} \mathrm{C}$, secondary antibodies for 1 hour at room temperature. Blots were scanned using the LI-COR Odyssey and analyzed with Image Studio software.

Immuno-TEM. Performed as previously described. ${ }^{38}$

Co-immunoprecipitation. Fractions from the VCCC were isolated as previously described by us ${ }^{10,38}$ and immunoprecipated as previously described by us..$^{38}$

Blood cell indices. Retro-orbital bleeds were taken with capillary tubes from EC Calr fl/fl and EC Calr $\Delta / \Delta$ mice and transferred into EDTA-containing vacutainer tubes. Samples were sent to the University of Virginia Health System's Clinical Pathology laboratory for complete blood cell analysis.

$q R T-P C R$. RNA was isolated from snap frozen thoracic aortas and interscapular brown adipose tissue using the RNeasy Lipid Tissue Kit or the RNeasy Mini Kit (Qiagen). Tissue was homogenized using ceramic beads in the Precellis system, 30 seconds at 5000rpm at $4^{\circ} \mathrm{C}$. Once homogenized, chloroform was added and mixed. The aqueous phase was taken and mixed with $70 \%$ ethanol and transferred to an RNeasy spin column where the rest of the procedure took place. RNA concentration was determined via NanoDrop and stored at $-80^{\circ} \mathrm{C}$ until copyDNA (cDNA) synthesis. The cDNA was used as a template for qPCR with the Go Taq qPCR Master Mix (SYBR green). Mouse primers (Sigma) were used for 
IP3R1 (Forward:AGCAGAATGAGACAGAAAAC

Reverse:TGGTTGAATGTAAAACCAGG),

SERCA2 (Forward:AATTGGAGAAGTGCAAAAGG,

Reverse:TACATTCATCTTCTCCACCAG),

PECAM (Forward: CATCGCCACCTTAATAGTTG,

Reverse:CCAGAAACATCATCATAACCG),

Bip (Forward: CAAGGTCTATGAAGGTGAAC,

Reverse:CACTCGGAGAATACCATTAAC),

Calr (Forward:AAAATCCTGAATACAAGGGC,

Reverse:CATAGATATTTGCATCGGGG)

B2M (Forward:GTATGCTATCCAGAAAACCC,

Reverse:CTGAAGGACATAATCTGACATC).

Samples were run using Applied Biosystems Step 1 Real Time PCR system and software. All data presented was run in duplicate, normalized to B2M and analyzed using the $\mathrm{X}_{0}$ method.

Histology. Third order mesenteric arteries were excised, fixed in $4 \%$ paraformaldehyde and embedded in paraffin blocks. Transverse sections of the arteries were stained with trichrome to visualize collagen staining or hematoxylin and eosin to visualize general structure and cellular nuclei.

In silico modeling and peptide generation. A GRAMM-X docking simulation ${ }^{175}$ was run for calreticulin and ITPKA/Kif18B using chains PDBid: 5LK5.I (CALR), 1W2C.A (ITPKA) and 3LRE.A (KIF18A). Thirty poses were obtained and interacting residues were analyzed using the InterfaceResidues script available on the PyMOL Wiki. 
Interacting residues were analyzed for reoccurring motifs, and the peptide candidate was chosen based on prevalence in the possible interaction motifs and possible binding modes due to the relatively unconstrained secondary structure. The peptide sequence was YGRKKRRQRRR (TAT)-PEAPTEEEHA (ITPKA peptide) and TAT-VQGLSFHQPAS (KIF18B peptide).

Statistical analysis. Data is presented as mean \pm standard error of the mean. Graph Pad Prism software was used for statistical analysis. Unpaired student's t-test was used to compare groups of 2. One way ANOVA was used for immunofluorescent stains and GCaMP analysis. For calcium analysis, paired t-tests were used to compare baseline and drug treatment from the same artery. A $P$ value of $<0.05$ was considered statistically different, unless otherwise noted. 
CHAPTER 4. A CELL CULTURE MODEL OF RESISTANCE ARTERIES 


\subsection{Abstract}

The MEJ, a unique signaling microdomain in small diameter resistance arteries, exhibits localization of specific proteins and signaling processes that can control vascular tone and blood pressure. As it is a projection from either the endothelial or smooth muscle cell, and due to its small size (on average, an area of $\sim 1 \mu \mathrm{m}^{2}$ ), the MEJ is difficult to study in isolation. However, we have developed the VCCC model that allows for in vitro MEJ formation, endothelial cell polarization, and dissection of signaling proteins and processes in the vascular wall of resistance arteries. The VCCC has a multitude of applications and can be adapted to suit different cell types. The model consists of two cell types grown on opposite sides of a filter with $0.4 \mu \mathrm{m}$ pores in which the in vitro MEJs can form. Here we describe how to create the VCCC via plating of cells and isolation of endothelial, MEJ, and smooth muscle fractions, which can then be used for protein isolation or activity assays. The filter with intact cell layers can be fixed, embedded, and sectioned for immunofluorescent analysis. Importantly, many of the discoveries from this model have been confirmed using intact resistance arteries, underscoring its physiological relevance. 


\subsection{Introduction}

A mechanistic dissection of signaling pathways at the MEJ is difficult in an intact artery. Because the MEJ is a cellular projection, it is not currently possible to isolate an in vivo MEJ from the vascular wall. For this reason, the vascular cell co-culture model (VCCC $)^{17}$ was developed. Importantly, the VCCC replicates physiological endothelial morphology $y^{176}$ and polarization of signaling between the apical and MEJ portions of the cell. ${ }^{10}$ It was this unique model that facilitated the discovery that alpha hemoglobin is in the endothelial cell, polarized to the MEJ. This is in contrast to conventionally cultured endothelial cells which do not express alpha hemoglobin. ${ }^{38}$ For researchers interested in microvascular endothelium, it may be more appropriate to use endothelial cells that have been cultured in the VCCC, particularly if the intent is to dissect signaling pathways that occur in small diameter resistance arteries.

Using a sturdy plastic insert containing a filter with small diameter pores to coculture two distinct cell types prevents the cells from migrating between layers. It results in a distance of $10 \mu \mathrm{m}$ between the cells, which is significantly longer than in vivo MEJs, but still replicates many of the in vivo characteristics of MEJs, including protein localization and second messenger signaling ${ }^{14,17}$. In addition, the VCCC allows for targeting of cell type-specific signaling via addition of an agonist or antagonist to the specific cellular compartment. For example, loading the EC with 1,2-bis(oaminophenoxy) ethane- $N, N, N^{\prime}, N^{\prime}$-tetraacetic acid (BAPTA-AM) to chelate calcium, and stimulating the SMC with phenylephrine. ${ }^{14}$ In contrast to other descriptions of co-culture models ${ }^{177-183}$, this provides instructions on isolating the distinct fractions for mRNA and protein, including the distinct MEJ fraction within the filter pores. The addition of this 
technique to the VCCC allows for specific investigation of changes in mRNA

localization or transcription ${ }^{146}$, protein phosphorylation ${ }^{10,11}$ and protein activity assays ${ }^{10}$. This article will describe plating of EC on top of the filter and SMC on the bottom, although it is also possible to culture the two cell types in different conformations. ${ }^{176,180,}$ 184 


\subsection{Creation and isolation of the VCCC}

\section{Plating Cells for the VCCC}

1.1. Culture large $225 \mathrm{~cm}^{2}$ flasks of EC and SMC under sterile conditions at $37{ }^{\circ} \mathrm{C}$, until $70-90 \%$ confluent. Ensure that more EC than SMC are used; if using primary human EC and SMC, typically 3 large flasks of EC to 1 large flask of SMC.

1.1.1. Use primary cells at lower passages and do not use past passage 10. Choose the culture media based upon cells to be co-cultured. For primary human coronary artery EC, use EBM-2 EC basal media with EGM2 MV growth factors. For primary human coronary SMC, use SmBM SMC basal media with SmGM-2 growth factors. Prior to use with cultured cells, add growth factors to the media.

1.2. Construction of the VCCC Day 1: Spray large $(150 \mathrm{~mm})$ Petri dishes and lids with disinfectant (e.g., Cavicide) and wipe away with a paper towel or lint-free wipes. Then spray the Petri dishes with $70 \%$ ethanol $(\mathrm{EtOH})$ and place them in the hood to air dry.

1.3. Open the plate of filter inserts under sterile conditions, and coat the SMC side (bottom side of filter) of the insert with fibronectin solution. Keeping the insert in the provided 6well plate, pipet the fibronectin solution through the side slits to the bottom of the plate, making sure that the bottom half of the filter is covered (no more than $1 \mathrm{~mL}$ ).

1.4. After $30 \mathrm{~min}$ of treatment with fibronectin solution at room temperature in the cell culture hood, take inserts out of the plate, vacuum any excess solution, and invert, placing into the bottom half of the clean Petri dish. Set aside. Be sure not to disturb the filter insert membrane when suctioning excess solution; this can be avoided by gently tilting the plate, and vacuuming the solution off the edge of the insert.

1.5. Trypsinize a $225 \mathrm{~cm}^{2}$ flask of SMC with $3 \mathrm{~mL}$ of pre-warmed $2 \mathrm{X}$ Trypsin-EDTA 
solution, and once the cells have lifted off the plate, add $9 \mathrm{~mL} \mathrm{SMC} \mathrm{media} \mathrm{to} \mathrm{neutralize}$ the trypsin (3:1, media:trypsin). Transfer to a conical tube, mix well, and pipet $10 \mu \mathrm{L}$ onto a hemocytometer to determine cell number.

1.5.1. Count the number of cells in $5 \times 5$ boxes (i.e., 18), multiply this by 10,000 to get the number of cells in $1 \mathrm{~mL}(180,000)$. Then multiply by $12 \mathrm{~mL}$ (total volume of cell suspension, 2.16 million SMC). Ensure that there are enough SMC to plate the desired number of wells. Note: For example, 1 well should have 75,000 SMC and thus 18 wells would have 1.35 million total SMC. Divide 1.35 million cells needed by 180,000 cells per $1 \mathrm{~mL}$ and this results in $7.5 \mathrm{~mL}$ of the cell suspension that will be needed for plating. Then calculate the final volume needed ( 18 wells x $750 \mu \mathrm{L}=13.5 \mathrm{~mL}$ ). Thus, take $7.5 \mathrm{~mL}$ of cell suspension, bring the volume up to $13.5 \mathrm{~mL}$ with pre-warmed SMC media, and mix well.

1.6. Plate $750 \mu \mathrm{L}$ of the cell suspension containing approximately $75,000 \mathrm{SMC}$ onto the bottom side of each filter, being careful to not spill any of the media and cell solution. Place the lid on top and carefully transfer the Petri dish with the inserts into the incubator at $37{ }^{\circ} \mathrm{C}$ overnight. Note: The optimum cell density for other cell types will need to be empirically determined.

1.7. On day 2, fill clean 6-well dishes with $2 \mathrm{~mL}$ of fresh, pre-warmed SMC media. Remove the plates from the incubator. Take the inserts out one at a time and remove the media by suction, then place into the 6-well dish with SMC media.

1.8. Coat the opposite side of the filter (EC side) with $1 \mathrm{~mL}$ of a $0.5 \%$ bovine gelatin solution for at least $30 \mathrm{~min}$ at $37{ }^{\circ} \mathrm{C}$.

1.9. Trypsinize the $225 \mathrm{~cm}^{2}$ flask(s) of EC with $3 \mathrm{~mL}$ of pre-warmed $2 X$ Trypsin-EDTA 
(10X diluted in sterile Dulbecco's PBS) solution, with gentle tapping of the flask in order to lift the cells off the plate. Note: It may be necessary to place the flask back into the incubator at $37{ }^{\circ} \mathrm{C}$ for $1-3 \mathrm{~min}$.

1.9.1. Once the cells have lifted off the plate, add $9 \mathrm{~mL} \mathrm{EC} \mathrm{media} \mathrm{to} \mathrm{neutralize} \mathrm{the} \mathrm{trypsin}$ (3:1). Transfer to a conical tube, pool together, mix well, and pipet $10 \mu \mathrm{L}$ onto a hemocytometer to determine cell number.

1.9.1.1. Count the number of cells in $5 \times 5$ boxes (i.e., 60 ), multiply this by 10,000 to get the number of cells in $1 \mathrm{~mL}(600,000)$. Then multiply by $12 \mathrm{~mL}$ (total volume of cell suspension, 7.2 million EC). Ensure that there are enough EC to plate the desired number of wells. Note: Approximately 360,000 EC are required per well. For example, 18 wells require 6.48 million EC. Divide 6.48 million cells needed by 600,000 EC per $1 \mathrm{~mL}$ and this results in $10.8 \mathrm{~mL}$ of the cell suspension that will be needed for plating. Then calculate the final volume needed ( 18 wells $x 1 \mathrm{~mL}=18 \mathrm{~mL}$ ). Thus, take $10.8 \mathrm{~mL}$ of cell suspension, bring the volume up to $18 \mathrm{~mL}$ with pre-warmed $\mathrm{EC}$ media, and gently resuspend.

1.10. Plate $1 \mathrm{~mL}$ of $360,000 \mathrm{EC}$ onto the top side of each insert filter. Let the cells incubate undisturbed at $37{ }^{\circ} \mathrm{C}$ for $24 \mathrm{~h}$. Replace medium on Day 4. Harvest on Day 5 .

\section{Harvesting Fractions from the VCCC}

2.1. Perform the isolation in a $4{ }^{\circ} \mathrm{C}$ room and keep all instruments on ice.

2.2. Keep in mind the number of individual inserts being isolated and add lysis buffer to a $50 \mathrm{~mL}$ tube labeled MEJ (for the isolated filters). Then label two 10-mm dishes; one for EC and one for SMC.

2.3. Adjust the volume of lysis buffer depending on how concentrated the lysate needs to be; for 15 inserts, $500 \mu \mathrm{L}$ of lysis buffer for the MEJ tube and $250 \mu \mathrm{L}$ per Petri dish is 
recommended.

2.4. Work with one 6-well plate of inserts at a time. Suction off the medium in the cell culture hood and then transport to a cold room on ice.

Note: The following instructions are for 1 insert isolation.

2.5. Pipet $10 \mu \mathrm{L}$ of $1 \mathrm{x}$ PBS onto the SMC side of the insert.

2.6. Use the cell lifter to scrape down the cells to one end of the insert, and transfer the cells from the lifter to the SMC Petri dish that has lysis buffer in it.

2.7. Once the cell lifter has touched the lysis buffer in the dish, do not allow it to touch the insert filter until it has been completely wiped and dried off on paper towels. Repeat the scraping of the SMC filter at least one to two times to fully remove the remaining SMC cell debris.

2.8. Turn the insert over and pipet $10 \mu \mathrm{L}$ of $1 \mathrm{x}$ PBS into the upper/EC side of the insert filter. Repeat steps 2.6 and 2.7 with a cell scraper and the EC Petri dish.

2.9. Collect the remaining cell and PBS slurry from the EC side of the filter with a pipette and transfer to the EC Petri dish with lysis buffer. Be very thorough in scraping the cells off both sides of the filter as to leave minimal EC/SMC contamination in the MEJ fraction. 2.10. Carefully use the scalpel to cut out the filter from the plastic insert. Do this by cutting $70-80 \%$ of it away from the plastic and use forceps to pull the filter completely off the plastic insert structure. Put the filter in the $50 \mathrm{~mL}$ conical tube labeled MEJ with lysis buffer, and ensure it sits in the lysis buffer and remains wet.

2.11. Repeat the isolation steps, in groups of 6 , until all of the inserts have been processed. 2.12. Ensure that different treatment groups are kept in separate tubes and dishes.

2.13. Vortex the $50 \mathrm{~mL}$ MEJ conical tube on full strength for $15 \mathrm{~s}$ or until mixed well. 
Remove the filters from the MEJ conical with forceps, dragging them along the side walls of the tube as to leave the maximum amount of proteins and liquid in the tube. After this point, discard the filters.

2.14. Once all the filters have been removed from the MEJ conical tube, do a quick spin (10-15 s at max speed $\sim 16,000 \mathrm{xg}$ ) in a centrifuge to pull all the liquid and proteins to the bottom of the tube. Transfer the contents of the MEJ tube and the EC and SMC Petri dishes into separate, smaller centrifuge tubes.

2.15. Optional: Sonicate the contents of the MEJ/SMC/EC tubes on setting 4 for three 10 s pulses on ice or homogenize gently by hand.

2.16. Centrifuge at max speed $(\sim 16,000 \mathrm{xg}) 15 \mathrm{~min}$ at $4{ }^{\circ} \mathrm{C}$. Pipet out the supernatant and assay the lysate for protein content using the bicinchoninic assay method.

\section{Harvesting the VCCC for En Face Immunofluorescence}

3.1. On Day 5 of VCCC incubation, remove the inserts from the incubator. Working in the cell culture hood, suction off the SMC media from each of the lower wells of the insert filter and rinse two times with 1x PBS. Repeat with the EC side.

3.2. Keeping the inserts intact and in the plate, add 4\% paraformaldehyde (PFA) for 10-20 min in a walk-in $4{ }^{\circ} \mathrm{C}$ room on a gentle shaker. Wash both sides of the insert filter with $1 \mathrm{x}$ PBS for 5 min and repeat two times.

3.3. Perform the blocking, primary and secondary antibody incubations in the plate, ensuring that both sides of the filter are kept in the antibody + blocking solution $(9 \mathrm{~mL}$ PBS, $25 \mu \mathrm{L}$ Triton X100, $50 \mathrm{mg}$ bovine serum albumin, $500 \mu \mathrm{L}$ normal serum)

3.4. To mount the filter on a slide, cut the filter out of the plastic insert with a scalpel mounted on a handle and place on a microscope slide with mounting medium. 


\section{Harvesting the VCCC for Transverse Immunofluorescence}

4.1. On Day 5 of VCCC incubation, remove the inserts from the incubator. Suction off the media from both sides of the insert filter in the cell culture hood and rinse each side of the filter two times with 1x PBS.

4.2. Keeping the inserts intact and in the 6-well plate, add 4\% PFA and incubate overnight in a walk-in $4^{\circ} \mathrm{C}$ room on a gentle shaker.

\section{Embedding the VCCC for Transverse Immunofluorescence}

5.1. After filter inserts have been fixed approximately for $24 \mathrm{~h}$ in $4 \%$ PFA, transfer to $70 \%$ EtOH for at least $24 \mathrm{~h}$, but up to several weeks.

5.2. Process the filters on a long (overnight) run on an automated tissue processor. Use the program as follows: $70 \% \mathrm{EtOH}$ for $30 \mathrm{~min}, 85 \% \mathrm{EtOH} 30 \mathrm{~min}, 95 \% \mathrm{EtOH} 30 \mathrm{~min}, 100 \%$ EtOH 30 min, 100\% EtOH 45 min, 100\% EtOH 45 min, Xylene 30 min, Xylene 30 min, Xylene $45 \mathrm{~min}, 60{ }^{\circ} \mathrm{C}$ paraffin $30 \mathrm{~min}, 60{ }^{\circ} \mathrm{C}$ paraffin $30 \mathrm{~min}, 60^{\circ} \mathrm{C}$ paraffin $45 \mathrm{~min}$.

5.3. After processing, transfer the harvested filters to the embedding station into liquid paraffin, keeping the filter in the cassette.

5.4. Remove the filter from the cassette, carefully using forceps to hold it just at the edge. With scissors, cut the filter in half, forming two semi-circular shaped halves. Lay each of the 2 halves on the cold plate portion of the embedding station just long enough to fill the embedding mold with liquid paraffin.

5.5. Once filled with liquid paraffin, very briefly touch the embedding mold to the cold plate, then embed each half of the filter into embedding mold (cut side down); this ensures that during sectioning, one will be cutting from the center of the filter towards the edge. 5.6. During embedding, use forceps to hold the filter in place to prevent the halves from 
falling into each other until the paraffin is cool enough for them to stand alone. Move the embedding mold to a cold plate until completely solidified.

Note: Cooling blocks on ice trays helps to prevent wrinkled sections.

\section{Sectioning and Staining the VCCC for Transverse Immunofluorescence}

6.1. For the paraffin blocks sectioning and slide mounting: cut $5 \mu \mathrm{m}$ sections, place the sections into a $42{ }^{\circ} \mathrm{C}$ water bath, and pick up the sections using positively charged slides. Then keep slides overnight in a $42{ }^{\circ} \mathrm{C}$ oven to dry.

6.2. Before beginning the rehydration steps, bake the slides at $60{ }^{\circ} \mathrm{C}$ to melt the paraffin and help adhere the VCCC sections to the slide. Cool briefly, then rehydrate the slides through two changes of xylene, $100 \% \mathrm{EtOH}, 95 \% \mathrm{EtOH}$, one change of $70 \% \mathrm{EtOH}$, and two changes of distilled water.

6.3. Avoid antigen retrieval methods that involve microwaving or heating as sections may fall off the slide.

6.4. Perform the standard blocking for $1 \mathrm{~h}$ at room temperature, then the overnight incubation with the primary antibody at $4{ }^{\circ} \mathrm{C}$, followed by washing steps and secondary antibody for $1 \mathrm{~h}$ at room temperature. Mount with the mounting media and coverslip. 


\subsection{Representative results}

The filter inserts used for the VCCC have small, $0.4 \mu \mathrm{m}$ holes. Figure 3A, an en face view of the VCCC filter inset, shows the pores in which the MEJ can form in vitro. The schematic depicts the smooth muscle cells plated on the lower side of the filter one day before the endothelial cells are plated on the opposite side (Figure 3B). Once the cells are plated, the EC, MEJ, and SMC fractions can be isolated three days later. An example of this is shown where alpha globin (Figure 4B) and plasminogen activator inhibitor 1 (PAI-1; Figure 4C) are highly expressed in the MEJ fraction relative to the rest of the EC. An in vivo MEJ in Figure 4A shows alpha globin enrichment at the MEJ (visualized as black dots). These data demonstrate the utility of the VCCC in recapitulating the intact arteriolar blood vessel wall. 


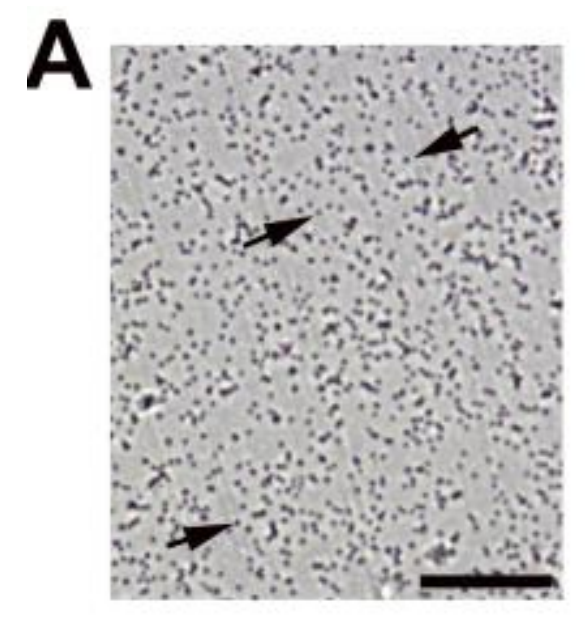

\section{B}

\section{Smooth Muscle}

Cells

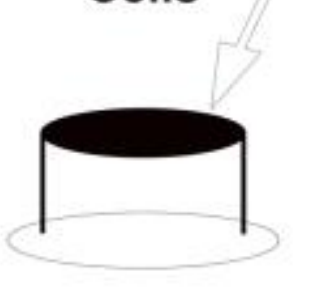

Day 1

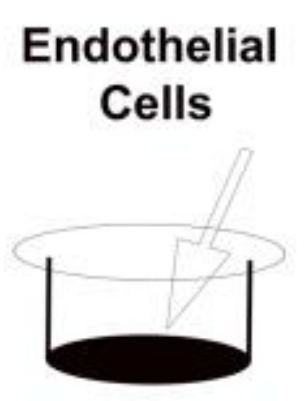

Day 2

Figure 3. Plating of the vascular cell co-culture (VCCC). A. Light micrograph of the pores in the insert filter. Black arrowheads denote individual holes. B. Schematic of plating the VCCC. Day 1 shows inversion of the insert and plating SMC on the bottom. On day 2, the EC are plated on the opposite side of the filter and the VCCC remains in this conformation in a 6 well plate until harvest. White arrows indicate the side of the filter where the cells are plated and will grow. Scale bar $=1 \mathrm{~mm}$ 

(PAI-1) EXPRESSION IN THE MEJ
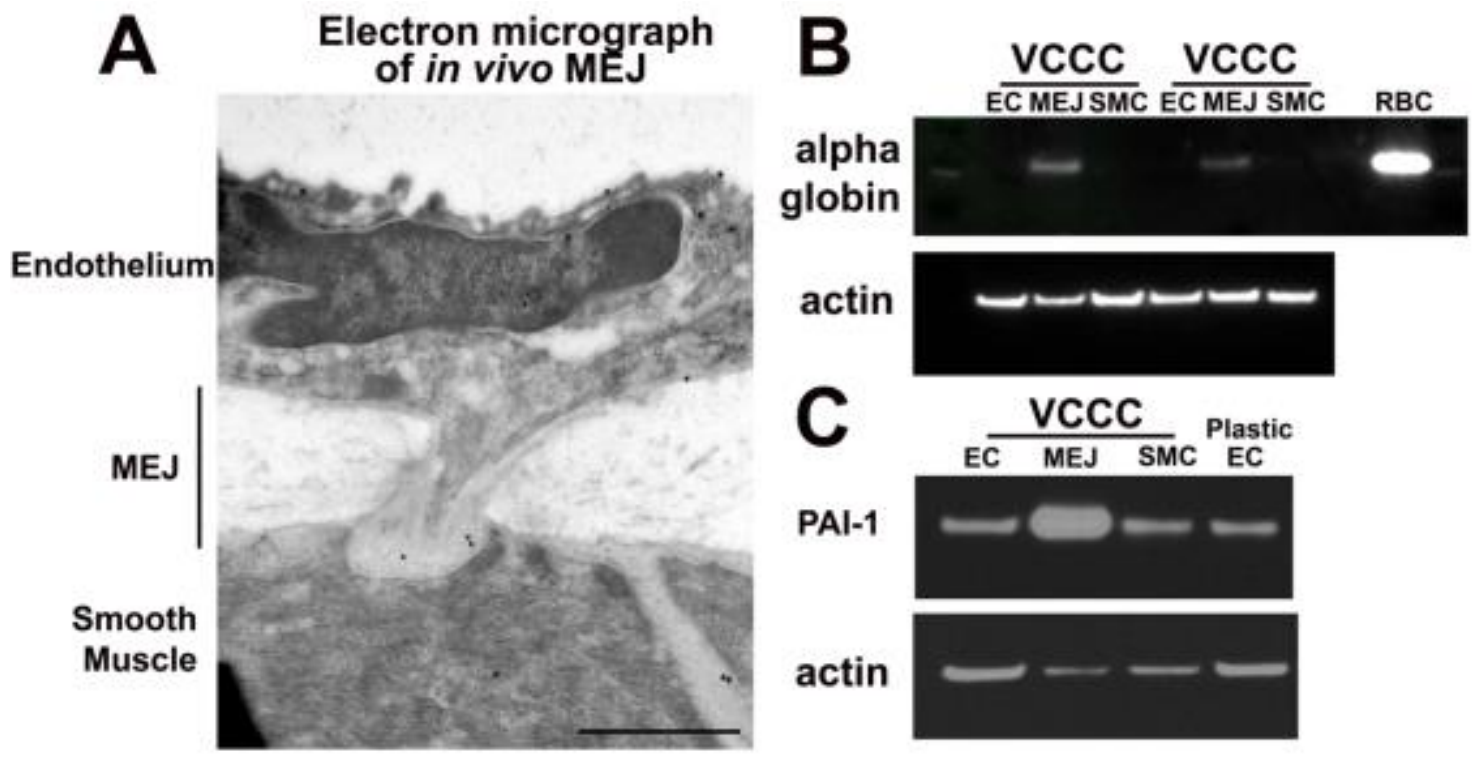

Figure 4. Alpha globin and plasminogen activator inhibitor (PAI-1) expression in the MEJ. A. Electron micrograph of in vivo MEJ showing alpha globin expression in the EC nucleus and MEJ via gold beads (black dots). B. Western blot showing alpha globin expression is present primarily in the MEJ and red blood cells (RBC). C. Western blot showing PAI-1 expression is enriched in the MEJ fraction versus the EC or SMC fractions. 


\subsection{Discussion}

The VCCC has a number of advantages in terms of recapitulating MEJs in vitro, but there are points of discussion when determining if the VCCC can be utilized for specific application. For example, if different cell types are to be used with this model, it will need to be optimized. We have used primary human cells but it is possible to isolate cells from bovine aortas ${ }^{184}$ or wildtype or knockout mice and use them in the VCCC $^{14,17}$, 18 .

If using the VCCC for MEJ isolation, the most important technique to master is thorough scraping of the filter to ensure that the purity of MEJ fraction is not altered by excess endothelial or smooth muscle cells. We have shown via western blotting that alpha hemoglobin is the best marker of a "pure" MEJ isolation" ${ }^{38}$, as it should be strongly expressed in the MEJ fraction versus the endothelial cell fraction. Another is plasminogen activator inhibitor 1 , which regulates the formation of MEJ. ${ }^{7}$ We feel these may be especially good markers for a robust MEJ isolation. For extra confidence in the harvest technique, the filters may be kept after scraping for harvest and fixed in PFA and stained for EC/SMC markers.

Imaging protein expression in the VCCC can be performed in two main ways: en face (Figure 2, 3) or transverse (Figure 5). Both preparations allow for the visualization of proteins within the holes of the filter from two different perspectives. The en face technique involves arteries that are cut open longitudinally and then pinned out flat, with the EC facing up, to visualize both the EC monolayer and the holes in the IEL, the only place where MEJ can form. A fluorescent signal within the holes of the IEL indicates localization of the protein within the $\mathrm{MEJ}^{13}{ }^{15}$. This same principle can be translated to 
the VCCC by imaging the EC monolayer from above, without needing to embed it in paraffin or make sections. The transverse method is more complex to master but is critical for distinct visualization of proteins in the EC versus the SMC monolayers (Figure 5).

The lack of flow or stretch in the system is a limitation, but it is possible to add flow in an endothelial-smooth muscle cell co-culture ${ }^{178,181}$. It appears that the static conditions still allow for physiologically accurate protein expression and activation, so it may be that the heterocellular contact is the most important factor in dissection of MEJ signaling pathways.

There are multiple applications of this technique beyond resistance artery signaling as the VCCC is not necessarily limited to specific cell types. Other co-culture models have utilized outgrowth endothelial cells and osteoblasts ${ }^{179}$, or modeled the blood brain barrier with endothelial and pericyte/astrocyte co-cultures ${ }^{183}$. Metastasis of tumor cells through the endothelium can also be quantified using this model ${ }^{183}$. It is also possible to grow cells on the filter and culture another cell type in the bottom of the 6well dish to test paracrine signaling, or grow endothelial cells on the upper part of the membrane to look at polarization of the cell (our unpublished observations). Leukocytes or other circulating cells can be added to the EC media and the adhesion of cells can be quantified $^{10}$. In addition, the VCCC can be used to investigate pathologies such as smooth muscle migration ${ }^{184}$ and proliferation in response to endothelial injury ${ }^{177,180}$. In conclusion, we have presented a method to isolate MEJ from an in vitro cell culture system, which allows for investigation of signaling processes between two coupled cell types. Importantly, the co-culture system is not limited to the study of the 
MEJ and can be valuable to answer number of questions, especially those involving heterocellular communication. 
CHAPTER 5. THE MYOENDOTHELIAL JUNCTION HAS A UNIQUE LIPID COMPOSITION THAT FACILITATES PROTEIN KINASE C ACTIVATION AND eNOS POLARIZATION 


\subsection{Abstract}

In resistance arteries, EC make contact with smooth muscle cells (SMC), forming MEJ. Endothelial nitric oxide synthase (eNOS) is present in the luminal side of the EC (apical EC) and the basal side of the EC (MEJ). To test if these eNOS pools acted in sync or separately, we co-cultured ECs and SMCs, then stimulated SMCs with PE. Adrenergic activation can cause $\mathrm{IP}_{3}$ to move from SMC to EC through gap junctions at the MEJ. PE increases MEJ eNOS phosphorylation (eNOS-P) at S1177, but not in EC. Conversely, we used bradykinin (BK) to increase EC calcium; this increased EC eNOS-P but did not affect MEJ eNOS-P. Inhibiting gap junctions abrogated the MEJ eNOS-P after PE, but had no effect on BK eNOS-P. Differential lipid composition between apical EC and MEJ may account for the compartmentalized eNOS-P response. Indeed, DAG and phosphatidylserine are both enriched in MEJ. These lipids are cofactors for PKC activity, which was significantly increased at the MEJ after PE. Because PKC activity also relies on ER calcium release, we used thapsigargin and xestospongin C, BAPTA, and PKC inhibitors, which caused significant decreases in MEJ eNOS-P after PE. Functionally, BK inhibited leukocyte adhesion and PE caused an increase in SMC cGMP. We hypothesize that local lipid composition of the MEJ primes PKC and eNOS-P for stimulation by PE, allowing for compartmentalized function of eNOS in the blood vessel wall. 


\subsection{Introduction}

Endothelial nitric oxide synthase (eNOS) is one of the most important enzymes found in EC that line the lumen of arteries and veins. Its ability to generate nitric oxide (NO) allows for control of vascular tone along with preventing inflammation and proliferation of the surrounding vascular smooth muscle cells (SMC) ${ }^{185-187}$. Alterations in eNOS activity or expression are linked to a number of cardiovascular pathologies that exhibit endothelial dysfunction, such as hypertension and inflammation ${ }^{188,} 189$.

Localization of eNOS within the EC has been shown in the perinuclear region (Golgi) and at the plasma membrane in vitro ${ }^{190}$ and in vivo ${ }^{191}$. Targeting eNOS to these domains requires lipid modifications such as acylation ${ }^{192}$. When eNOS is unable to be acylated, it remains in the cytosol instead of being targeted to the plasma membrane, hindering normal activation of signaling. These cellular regulatory processes are important as deviation from normal localization or modification renders eNOS unable to increase its activation via phosphorylation ${ }^{190}$. Under unstimulated circumstances, eNOS is sequestered in specialized lipid domains at the plasma membrane, which are characterized by the presence of cholesterol and caveolae, flask-shaped invaginations of the plasma membrane ${ }^{192}$. The coat protein of caveolae, caveolin 1, directly interacts with eNOS to maintain its quiescence ${ }^{193,194}$. However, increases in intracellular calcium cause membrane dissociation and subsequent translocation to the cytosol. Enzyme phosphorylation is increased at one of several activating sites, including S1177 in human EC, causing the production of NO.

Importantly, the MEJ has been shown to be a source of phosphorylated, activated eNOS that generates NO, regulating heterocellular communication and vascular tone ${ }^{11}$, 
20,38. The MEJ is a cellular projection, typically endothelial in origin, that allows for direct cytoplasmic contact with the smooth muscle cells that comprise the outer wall of small diameter arteries and arteries ${ }^{1}$. After stimulation of alpha $1 \mathrm{D}$ adrenergic receptors on $\mathrm{SMC}$ with $\mathrm{PE}, \mathrm{IP}_{3}$ can travel through MEJ-localized gap junctions and induce ER calcium release via local IP3R expression ${ }^{14,18,145}$, which increases EC production of NO ${ }^{23}$ via eNOS phosphorylation at serine 1177 (eNOS-P) ${ }^{195}$. Given the function of eNOS as a regulator of normal and pathological vascular physiology, we wanted to investigate the distinct subcellular characteristics and function of eNOS in EC that form MEJs. Using an in vitro MEJ model (VCCC), we show that there are two distinct pools of eNOS in the endothelium that are differentially activated. This has different functional consequences, which may be due to differences in lipid composition, along with protein kinase $\mathrm{C}$ (PKC) localization and activity at the MEJ. 


\subsection{Results}

We have previously demonstrated differential pools of eNOS in EC of resistance arteries: in the apical EC and at the portion of the EC making contact with the SMC, the MEJ ${ }^{11}$. These two areas of EC are physically distinct and an accumulation of evidence has now demonstrated that these two areas are also functionally distinct ${ }^{14,38,73}$. Because NO generation in EC must be tightly regulated, we hypothesized that eNOS activation, as demonstrated by eNOS phosphorylation at serine (S)1177 (eNOS-P), may occur locally. To test this concept, we utilized our well-described $\mathrm{VCCC}^{7,14,17}$ that crucially has EC directly linked to SMC by EC-derived MEJs via gap junctions (Figure 5A). eNOS phosphorylation at S1177--In an attempt to selectively activate eNOS, we first wanted to ensure receptors for EC or SMC agonists were only found in each cell type. Using immunocytochemistry, we found that BK receptor 2 was only found on EC and alpha 1D adrenergic receptors were only found on SMC, with eNOS found in both apical EC and at the MEJ (Figure 5A). To test that we could induce eNOS

phosphorylation in either apical EC or MEJ, we found that 1 minute after stimulation with either BK or PE increased eNOS-P that appeared to be either apical or MEJ in nature (Figure 5B). Because of this, we performed western blot analysis on each of the fractions from the VCCC and found that after BK stimulation, only apical EC could demonstrate an increase in eNOS phosphorylation and this was not evident at the MEJ (Figure 5C). However, after PE stimulation, an increase in MEJ eNOS phosphorylation occurred without a change in apical EC (Figure 5D). Because an increase in intracellular calcium alone can regulate eNOS phosphorylation, we pretreated both EC and SMC with the gap junction inhibitor 18-GA; this selectively inhibited eNOS phosphorylation at the 
MEJ after PE stimulation (Figure 6B), but not in apical EC after bradykinin stimulation (Figure 6A). Therefore, it appears that depending on whether EC or SMC is stimulated, eNOS is activated in distinct locations of the EC.

Lipid composition and PKC activity--Next, we wanted to understand how EC could selectively allow for eNOS phosphorylation in the apical or MEJ regions of the cell. Because the MEJ itself has been shown to be a unique signaling microdomain and has a highly curved physical structure, we assessed lipid composition of the cells in the VCCC and compared them to unpolarized EC and SMC from a single monolayer grown in a plastic flask. We found phospholipid composition of EC or SMC in co-culture has a unique profile, rendering it profoundly different from a monolayer of the same cells (Figure 8). There were no significant differences in structural lipids phosphatidylcholine, phosphatidylethanolamine or sphingomyelin at the MEJ, but cholesterol content at the MEJ was significantly decreased. Ceramide has been shown to activate eNOS phosphorylation via a calcium-independent pathway $^{196}$, however the ceramide levels at the MEJ are not significantly different from the rest of the EC. Moreover, the signaling lipid sphingosine-1-phosphate (S1P) has also been shown to activate eNOS via its receptors on vascular cells ${ }^{197,}{ }^{198}$. MEJ levels of S1P were significantly lower than the rest of the EC, but S1P signaling and expression or function of S1P receptors at the MEJ has not been investigated. When phosphatidylserine (PS) and diacylglycerol (DAG), direct lipid cofactors for PKC activity, were analyzed, we found them to be significantly enriched at the MEJ (Figure 7A, B). Concomitant with these results, we found PKC activity is significantly higher at the MEJ, and further increases after PE stimulation (Figure 7C). 
Calcium dependent MEJ eNOS phosphorylation--In addition to DAG and PS, conventional isoforms of PKC require $\mathrm{IP}_{3}$ stimulated calcium release for activation ${ }^{199,} 200$. The ER has been shown to be present at MEJ and is possibly an important component to the negative feedback of SMC induced constriction, ${ }^{14,18}$ Not surprisingly, the VCCC also was found to have expression of the ER marker calreticulin in EC, MEJ and SMC (Figure 9A, B). In order to demonstrate a possible role for the calcium sequestered in these ER projections on eNOS-P at the MEJ, the VCCC were incubated with BAPTAAM and then stimulated with PE. This almost completely blocked MEJ eNOS-P (a 92\% decrease). After stimulation with PE, ER calcium release contributes to eNOS-P at the MEJ as thapsigargin and xestospongin C significantly decreased eNOS-P. Importantly, the broad spectrum PKC inhibitor Gö-6983 significantly decreased MEJ eNOS-P after PE while the conventional isoform inhibitor Gö-6976 only trended in that direction (Figure 9C).

Functional contribution of differential pools of eNOS in endothelium. Based on the work above, it was clear that ECs tightly regulate eNOS activation either apically or at the MEJ, depending on the stimulus. However, it was unclear to what extent this may differentially regulate the functional effect of eNOS. It is well known that NO is a potent inhibitor of leukocyte adhesion to inflamed endothelial cells ${ }^{185}$ so we hypothesized the apical eNOS, regulated by BK stimulation, could selectively affect the ability of monocytes to adhere to the EC. To test this, we used the pro inflammatory cytokine TNF- $\alpha$ to initiate leukocyte adhesion to the EC layer of the VCCC and activated the different pools of eNOS with BK or PE. Adding TNF- $\alpha$ significantly increased adhesion of the calcein-loaded THP1 monocytes compared to control (Figure 10). Inhibiting eNOS 
with L-NAME significantly exacerbated adhesion compared to TNF- $\alpha$ alone.

Stimulation of EC with BK significantly decreased the degree of leukocyte adhesion which is consistent with localized eNOS activation and NO release. However, adding PE had no effect on leukocyte adhesion, consistent with the differences in localized eNOS phosphorylation observed above. Therefore, apical eNOS appears to be distinctly important in abrogating leukocyte adhesion, whereas as MEJ eNOS does not appear to have an effect.

In order to determine a functional role for the eNOS at the MEJ, we examined SMC cGMP production, which is generated after NO binds to soluble guanyl cyclase. We hypothesized that MEJ localized eNOS and its proximity to the SMC made it a much better regulator of SMC dilation than eNOS in the apical portion of EC. Application of PE caused a significant increase in cGMP production after 5 minutes in SMC (Figure 10C) that is abrogated in the presence of gap junction inhibition (Figure 10D). This is in line with the eNOS-P observed above. Importantly, cGMP production does not occur in SMC grown in a monolayer without MEJ formation (Figure 10E), underscoring the importance of the MEJ localized eNOS phosphorylation event. 
FIGURE 5. ENDOTHELIAL NOS AT SERINE 1177 IS SELECTIVELY PHOSPHORYLATED BASED UPON ENDOTHELIAL OR SMOOTH MUSCLE CELL RECEPTOR ACTIVATION.

A
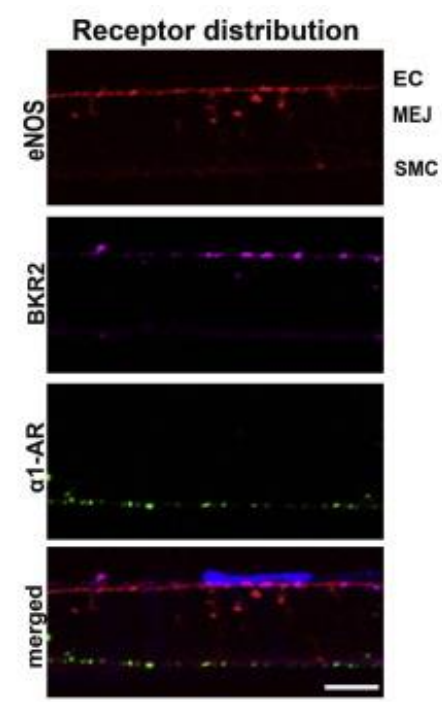

EC

C

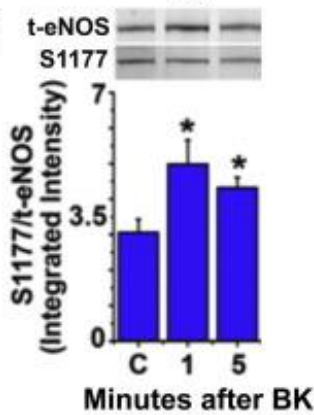

D

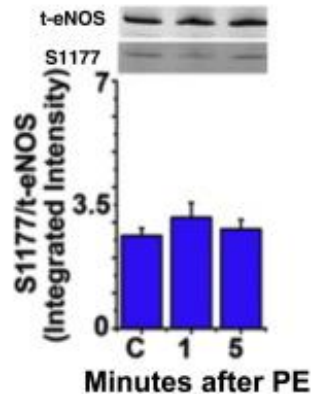

B phosphorylated eNOS (S1177)
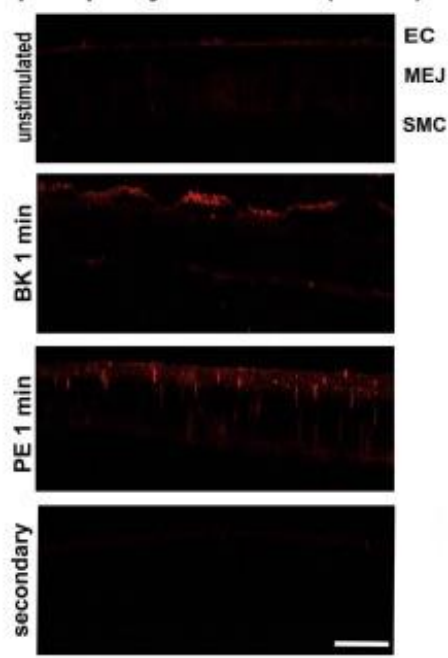

SMC
MEJ
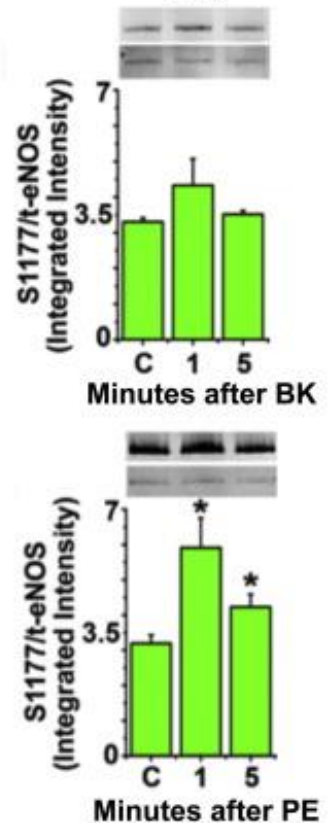
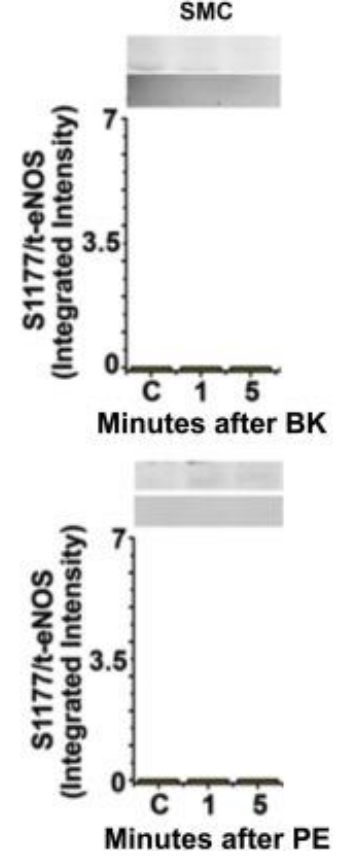
Figure 5. Endothelial NOS at Serine 1177 is selectively phosphorylated based upon endothelial or smooth muscle cell receptor activation. A, Transverse sections of the VCCC were incubated with antibodies to alpha 1 adrenergic receptor ( $\alpha 1 \mathrm{AR}$, green), bradykinin receptor 2 (BKR2, purple) and endothelial nitric oxide synthase (eNOS, red). SMC only shows $\alpha 1 \mathrm{AR}$ expression while EC only shows BKR2 expression. Endothelial NOS is found in the apical EC as well as the MEJ. B, Immunocytochemistry of VCCC with antibodies against phosphorylated eNOS at S1177 (eNOS-P, red) after stimulation with BK or PE for 1 min. C, Representative western blot of VCCC fractions after treatment with bradykinin $(\mathrm{BK}, 10 \mu \mathrm{M}, \mathrm{n}=4)$ or $\mathbf{D}$, phenylephrine $(\mathrm{PE}, 50 \mu \mathrm{M}, \mathrm{n}=5)$ at 1 and $5 \mathrm{~min}$ timepoints, with no treatment as control. The ratio of eNOS-P to total eNOS is presented. All error bars represent SEM. In C and D, * indicates $\mathrm{p}<0.05$. Scale bar for A and B is $10 \mu \mathrm{m}$. 
FIGURE 6. GAP JUNCTION INHIBITION PREVENTS PHENYLEPHRINE INDUCED ENOS PHOSPHORYLATION AT THE MEJ.
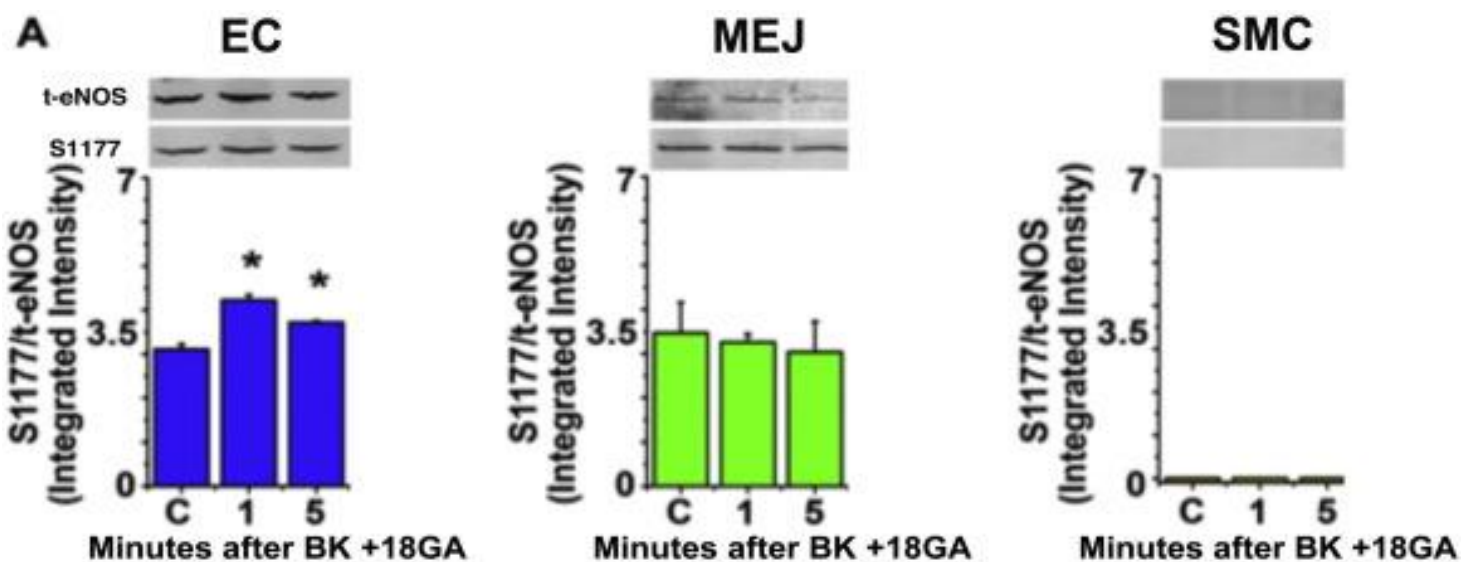

B
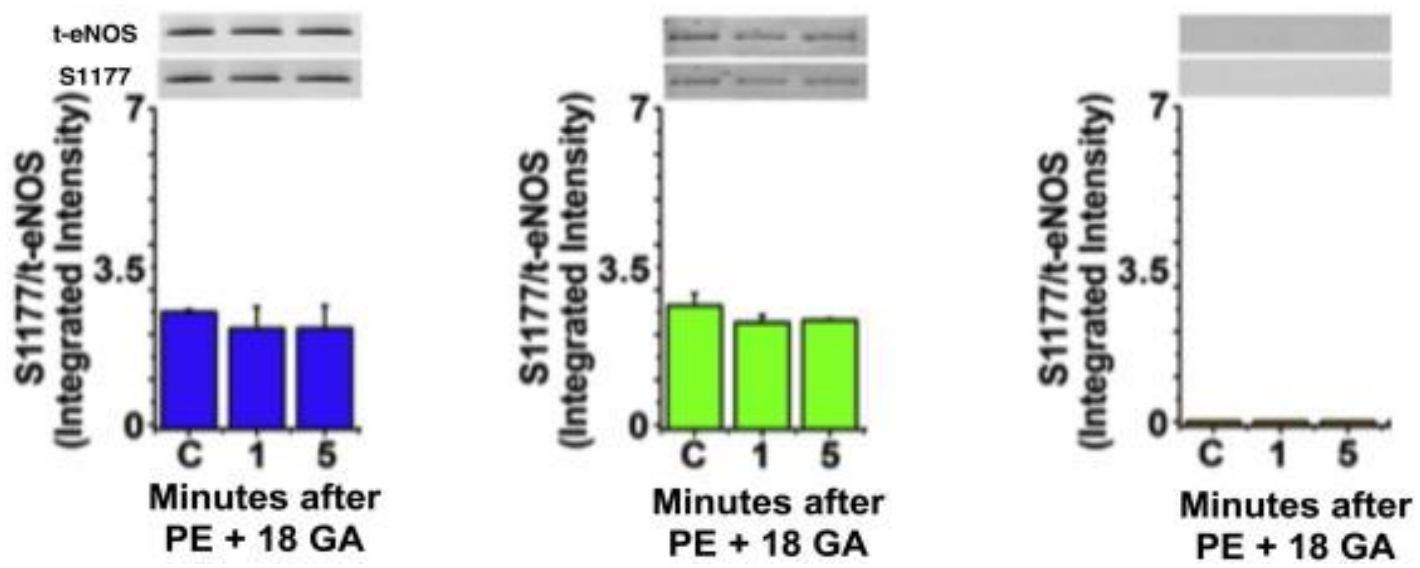

Figure 6. Gap junction inhibition prevents phenylephrine induced eNOS phosphorylation at the MEJ. The VCCC was incubated with the gap junction inhibitor 18-GA $(50 \mu \mathrm{M})$ for $30 \mathrm{~min}$ before adding bradykinin (BK, $10 \mu \mathrm{M}, \mathrm{n}=4)$ (A) or phenylephrine (PE, $50 \mu \mathrm{M}, \mathrm{n}=3$ ) (B). Timepoints indicate the minutes after stimulation. Cellular fractions were harvested for western blotting and the ratio of eNOS S 1177 to total eNOS is presented. All error bars represent SEM. In A and B, * indicates $\mathrm{p}<0.05$. 
FIGURE 7. DIACYLGLYCEROL AND PHOSPHATIDYLSERINE ARE

ENRICHED AT THE MEJ, FACILITATING A SIGNIFICANT INCREASE IN PKC ACTIVITY.
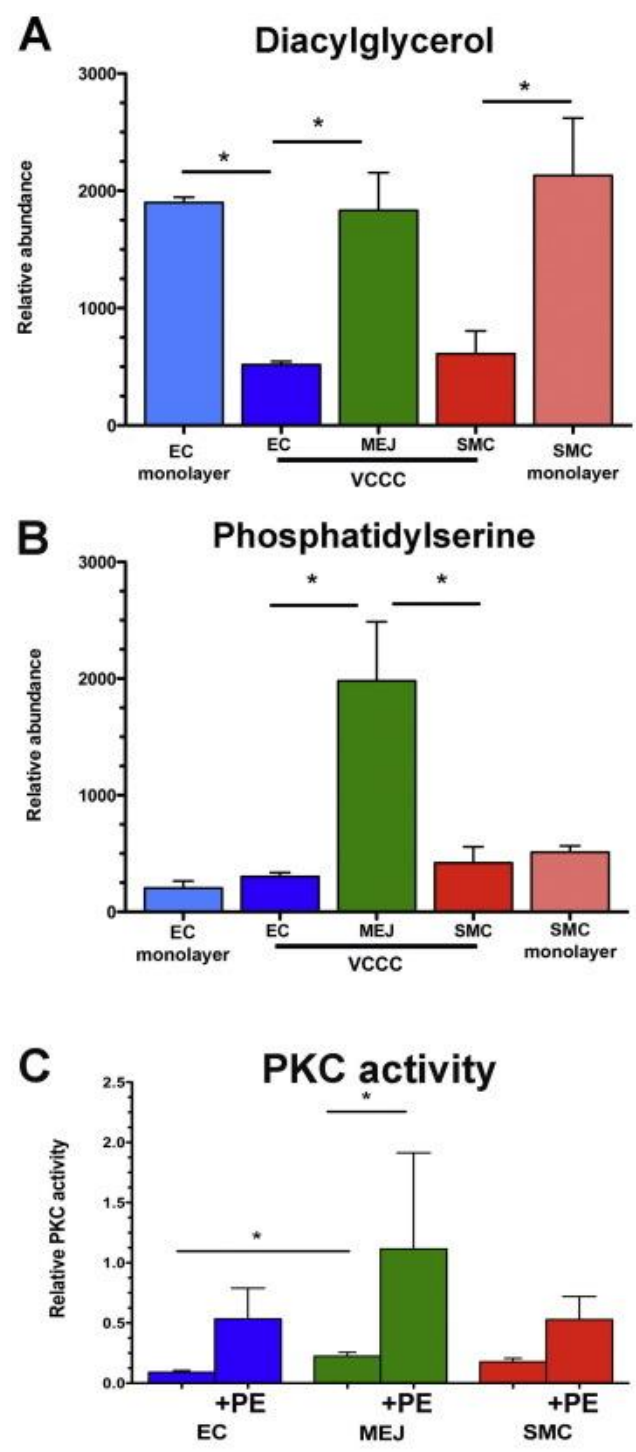

vccc 
Figure 7. Diacylglycerol and phosphatidylserine are enriched at the MEJ, facilitating a significant increase in protein kinase C. Glycerolipids $(n=3)$ were isolated from VCCC fractions and EC or SMC monolayers and analyzed via mass spectrometry (A, B). PKC activity measured in the VCCC $(n=3)(C)$. Lipid values were first normalized to an internal standard and then all values were normalized to protein concentration. Error bars represent SEM. * indicates $\mathrm{p}<0.05$. 
FIGURE 8. PRIMARY ENDOTHELIAL AND SMOOTH MUSCLE CELLS GROWN IN THE VCCC HAVE A UNIQUE LIPID COMPOSITION

A

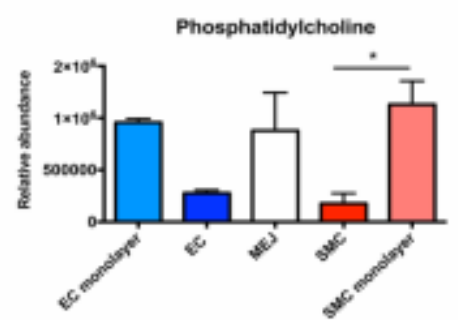

B

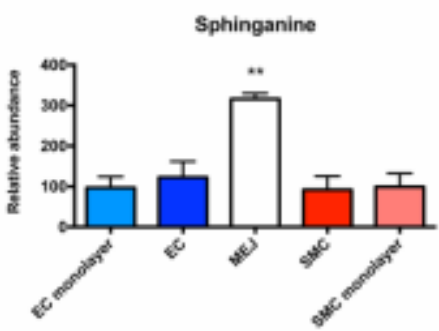

C

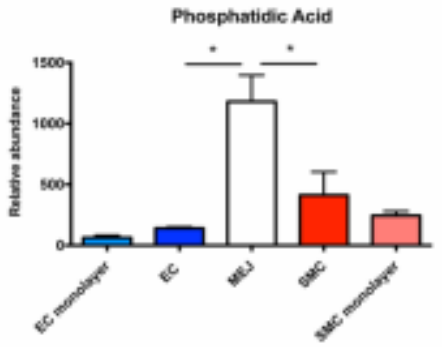

D

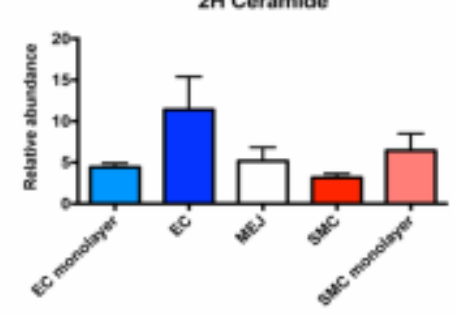

$\mathbf{E}$

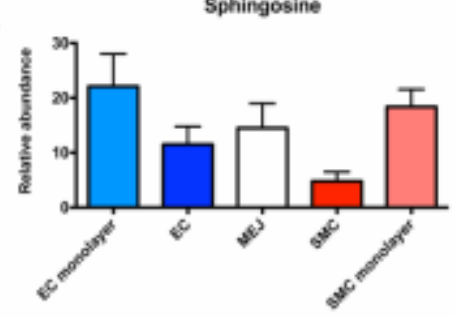

$\mathbf{F}$

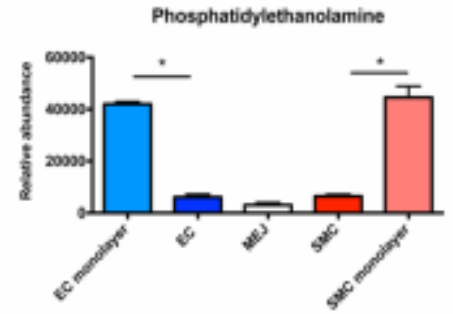

G

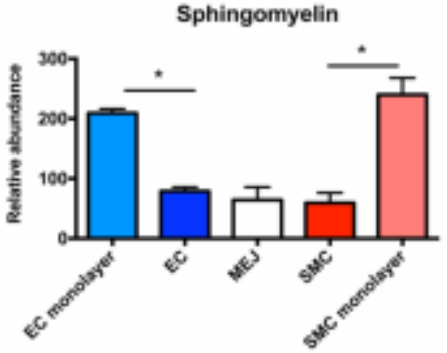

H

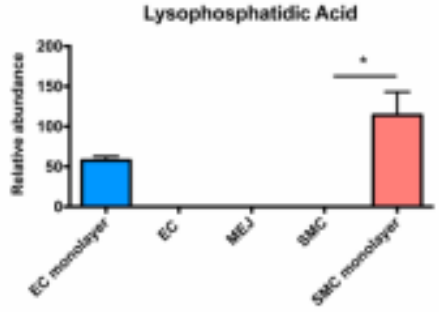

I

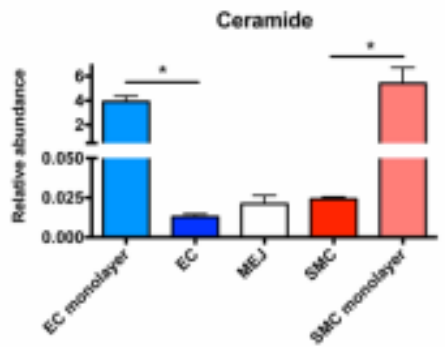

J

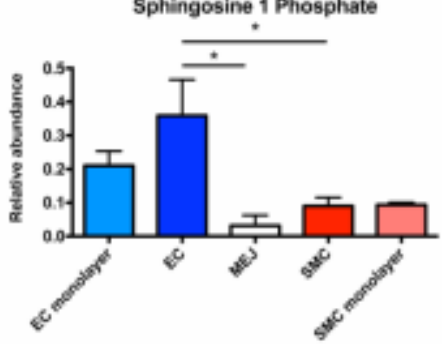


Figure 8. Primary endothelial and smooth muscle cells grown in the VCCC have a unique lipid composition. Glycerolipids $(\mathrm{n}=3)$ were isolated from VCCC fractions and EC or SMC monolayers and analyzed via mass spectrometry. EC and SMC monolayer fractions were isolated from cells grown in a plastic flask (in contrast to those grown on the VCCC). A, Phosphatidylcholine was higher in SMC monolayer versus SMC. B, Sphinganine and $\mathbf{C}$, Phosphatidic acid were significantly increased in the MEJ versus the other fractions. D, Dihydroceramide and E, sphingosine were not different between groups. F, Phosphatidylethanolamine, G, sphingomyelin, and $\mathbf{H}$, lysophosphatidic acid, I, were all decreased in EC and SMC from the VCCC. J, Phosphorylated sphingosine was significantly higher in the EC from the VCCC versus the MEJ and SMC. Error bars represent SEM. *p $<0.05$ 
FIGURE 9. ER IS PRESENT AT THE MEJ, INFLUENCING ENOS PHOSPHORYLATION.

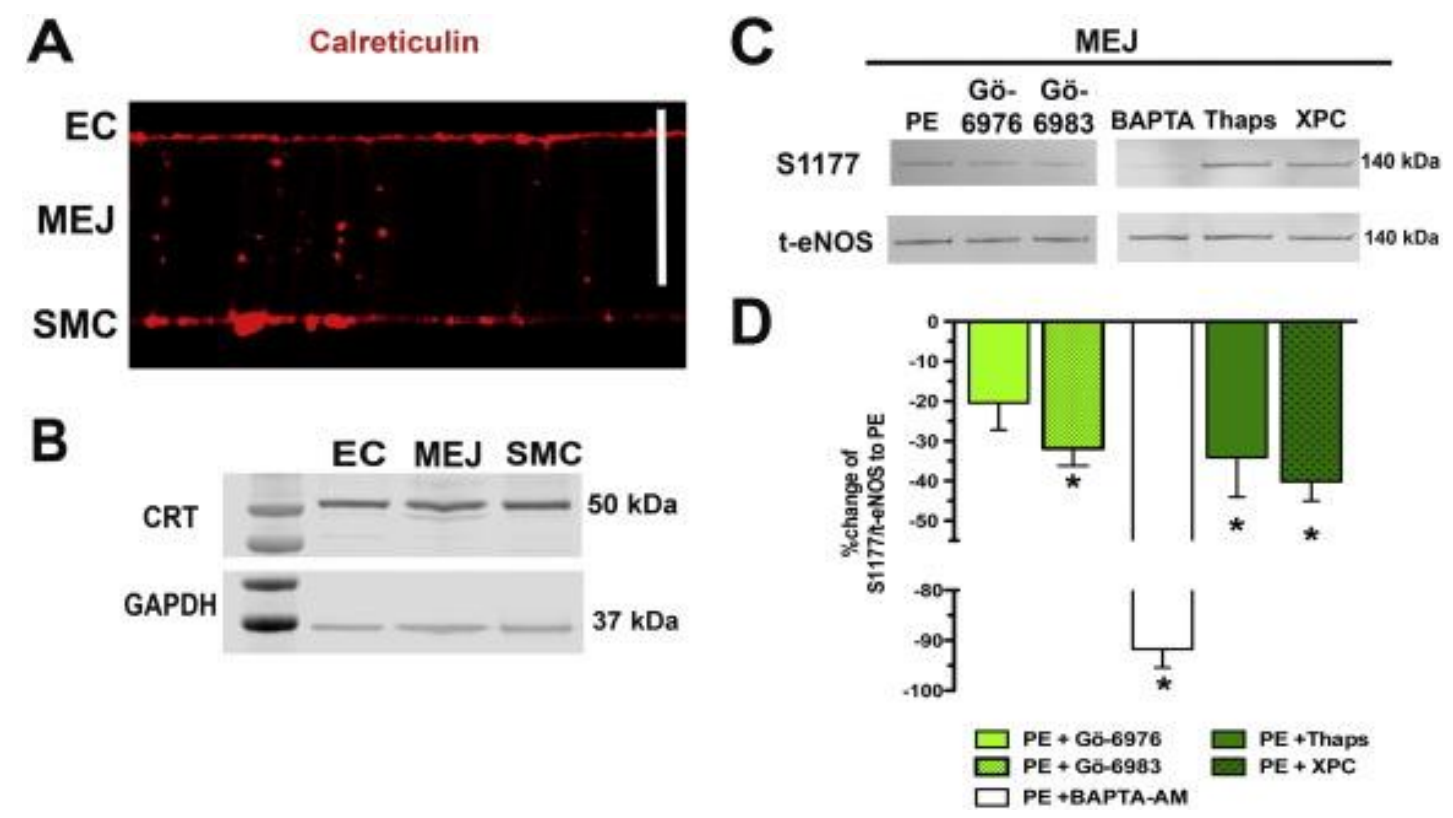

Figure 9. ER is present at the MEJ, influencing eNOS phosphorylation. A, Calreticulin, a class marker of the endoplasmic reticulum, was identified in the VCCC via immunocytochemistry and B, western blot. Scale bar is $10 \mu \mathrm{m} . \mathbf{C}$, Representative eNOSP to total eNOS blots for MEJ samples after stimulation with PE (50 $\mu \mathrm{M}, 1 \mathrm{~min}, \mathrm{n}=3)$ and incubation with Gö-6976 (10 $\mu \mathrm{M}, 30 \mathrm{~min}, \mathrm{n}=6)$, a classical PKC inhibitor, Gö-6983 (10 $\mu \mathrm{M}, 30$ min, $\mathrm{n}=5)$, a broad PKC inhibitor, BAPTA $(20 \mu \mathrm{M}, 20 \mathrm{~min}, \mathrm{n}=4)$ to chelate calcium, Thapsigargin $(2 \mu \mathrm{M}, 30 \mathrm{~min}, \mathrm{n}=4)$ to deplete ER calcium or Xestospongin $\mathrm{C}$ $(20 \mu \mathrm{M}, 10 \mathrm{~min}, \mathrm{n}=3)$ to inhibit $\mathrm{IP}_{3}$ receptor signaling. $\mathbf{D}$, The ratio of $\mathrm{S} 1177$ to total eNOS was compared back to the PE stimulated ratio. 
FIGURE 10. FUNCTIONAL EFFECTS OF APICAL VERSUS MEJ ENOS.

A

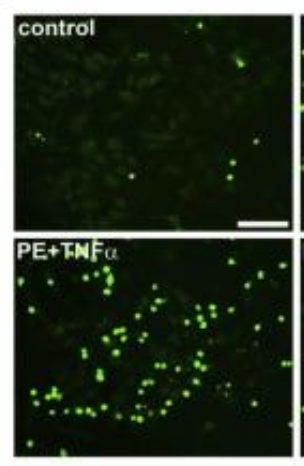

C

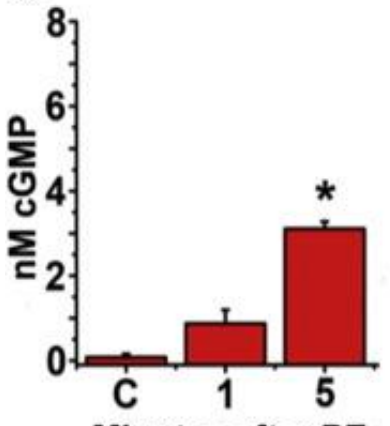

Minutes after PE

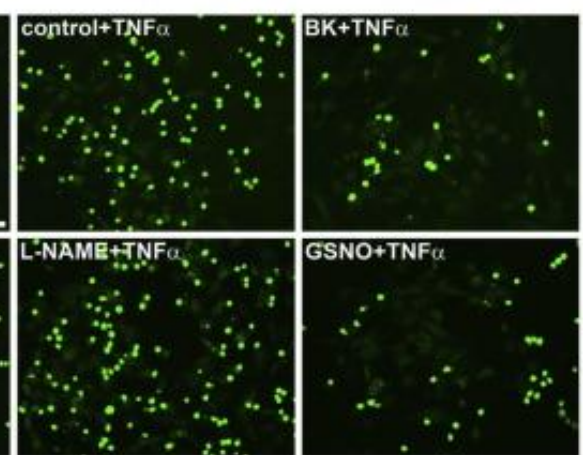

D



B

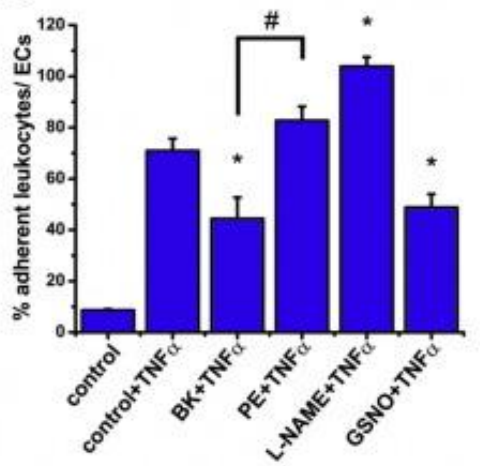

E

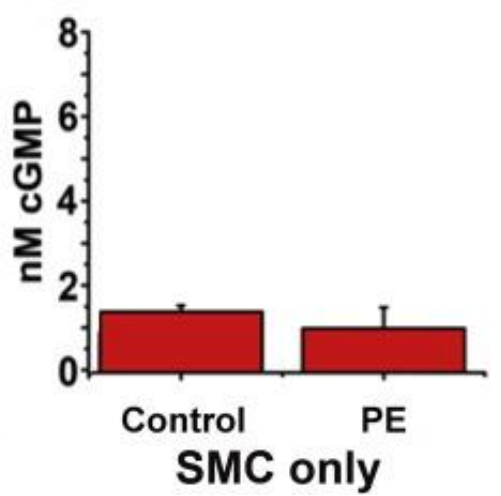


Figure 10. Functional effects of apical versus MEJ eNOS. A, Representative images of Calcein-AM (5 $\mu \mathrm{M})$ loaded THP1 monocytes (green) adhering to the EC side of the VCCC. The VCCC were incubated with TNF $\alpha$ for $4 \mathrm{~h}$ before adding BK $(1 \mu \mathrm{M}), \mathrm{PE}(50 \mu \mathrm{M})$ or GSNO $(100 \mu \mathrm{M})$ to the EC side for 1 min. A subset of VCCC had L-NAME $(100 \mu \mathrm{M})$ added at the same time as TNF $\alpha$. B, Data presented as the percentage of adherent THP1 monocytes to endothelial cells. C, The VCCC were stimulated with phenylephrine or $\mathbf{D}$, the gap junction inhibitor 18-GA prior to PE stimulation. E, As a control, after SMC were grown in a monolayer they were stimulated with PE and cGMP was measured. All error bars represent SEM. B: * indicates $\mathrm{p}<0.05$ vs control $+\mathrm{TNF} \alpha$, \# indicates $\mathrm{p}<0.05$ vs $\mathrm{BK}+\mathrm{TNF} \alpha . \mathrm{C}-\mathrm{E}: *$ indicates $\mathrm{p}<0.05$ versus control, $\mathrm{n}=4-6$. 
FIGURE 11. PROPOSED MECHANISM OF DIFFERENTIAL ENOS PHOSPHORYLATION IN ENDOTHELIUM.

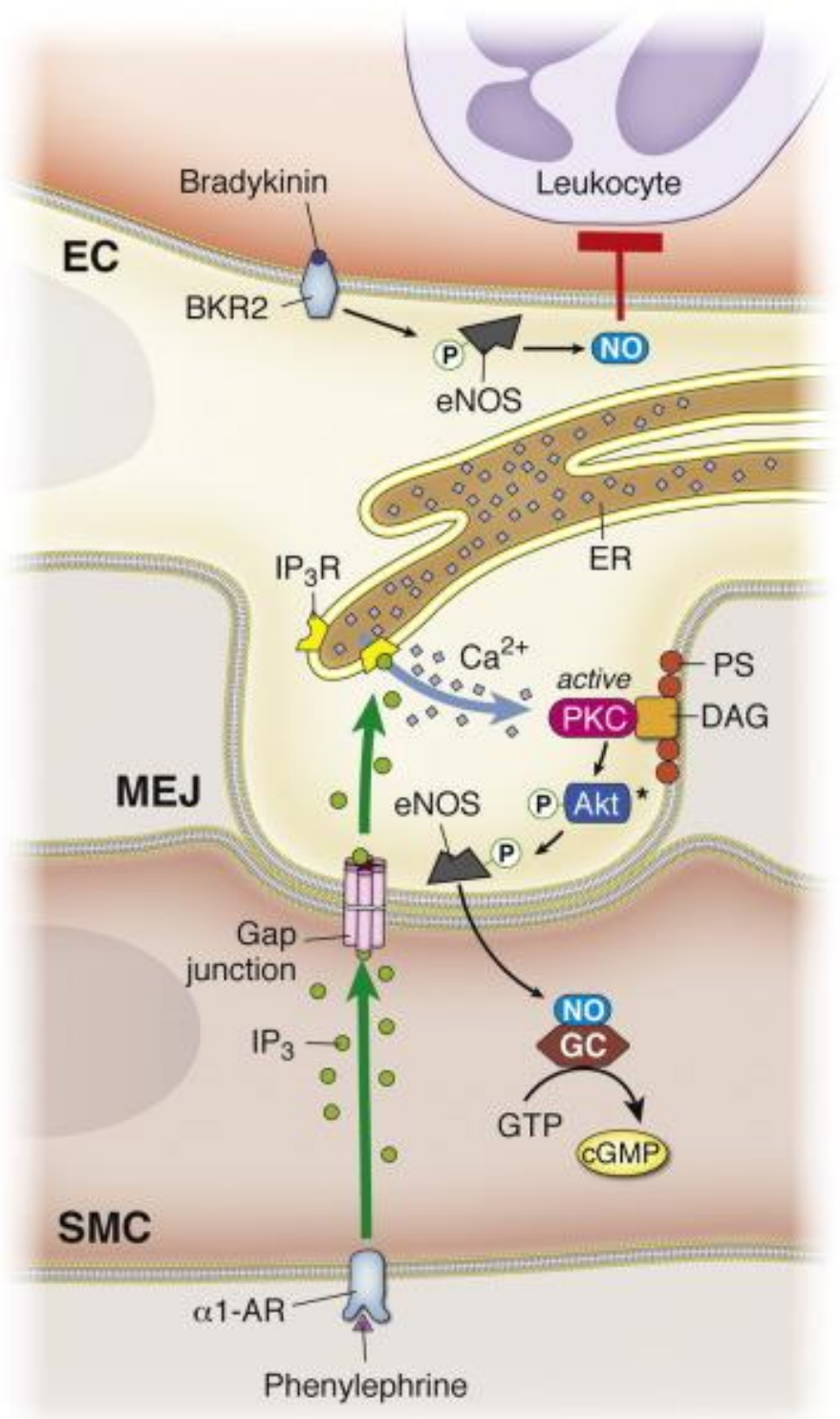


Bradykinin activation of the bradykinin receptor 2 (BKR2) causes an increase in eNOS-P in the apical endothelium, leading to significantly decreased leukocyte adhesion via production of anti-inflammatory NO generation. Phenylephrine activation of the $\alpha 1-\mathrm{AR}$ generates $\mathrm{IP}_{3}$, which can travel through gap junctions at the MEJ and activate localized $\mathrm{IP}_{3}$ receptors on the ER. Local ER calcium release contributes to MEJ eNOS-P via activation of MEJ localized PKC, which may occur via Akt. The unique phospholipid composition of the MEJ facilitates this localization of PKC via its lipid cofactors, PS and DAG. Activation of MEJ eNOS with subsequent production of NO leads to increases in SMC cGMP. 


\subsection{Discussion}

In this paper, we have shown the presence of two functionally distinct sources of eNOS in the endothelium. Despite the apical and MEJ eNOS similarity in basal levels of phosphorylation, they are activated differently depending on agonist stimulation. Based on the data presented, this may be due to differences in membrane lipid composition and kinase activity at the MEJ, which is underscored by the contribution of ER calcium. While the spatial localization of eNOS in the endothelium may have divergent functions (i.e. leukocyte adhesion versus production of SMC cGMP), this could be explained by the ability of the cellular microenvironment to control differential eNOS activation, thereby providing another level of NO control in the vascular wall (Figure 11).

Specific protein localization to the MEJ is important for cellular signaling and eNOS is a central mediator of signaling processes at the MEJ. ${ }^{201}$ Our lab has previously shown that MEJ-localized eNOS participates in S-nitrosylation of connexin 43 and this

allows the heterocellular transfer of small signaling molecules such as $\mathrm{IP}_{3} .{ }^{11}$ Connexins $(\mathrm{Cx})$ at cellular borders can also influence eNOS localization. Under normal cell conditions, Cx40 and Cx37 interact with eNOS ${ }^{202,203}$, but Cx40 knockout mice have decreased aortic eNOS expression. ${ }^{204}$ Although aortas have very few MEJs, the possibility that $\mathrm{Cx}$ may dictate eNOS localization in smaller diameter arteries with more MEJs could provide an explanation for the two spatial distinct pools of eNOS. Indeed, both Cx40 and Cx37 have been directly localized to MEJs. ${ }^{205}$

In addition to its ability to post-translationally modify proteins at the MEJ, we have shown that eNOS directly interacts with alpha hemoglobin to regulate NO diffusion at the MEJ. ${ }^{20,38}$ The localized presence of organelles such as peripheral ER with a 
thapsigargin/xestospongin $\mathrm{C}$ sensitive pool of calcium regulate the signaling processes at the MEJ by activating PKC and eNOS (Figure 10). The unique tubular structure of the peripheral ER (Chapter 2) allows for projection into the MEJ and local calcium release. IP3R localization at the MEJ ${ }^{14,145}$ facilitates the SMC to EC signaling cascade after PE stimulation of the alpha1D adrenergic receptor.

The subcellular localization of eNOS is strategically determined by the EC as the magnitude of eNOS activity is dependent on where the enzyme is present. For example, nuclear directed eNOS is significantly less phosphorylated ${ }^{206}$, while eNOS targeted to the plasma membrane generates the most NO, likely via its close association to membrane bound kinases. ${ }^{207}$ As eNOS-generated NO is a small gaseous molecule that can act in a local manner ${ }^{206}$, it corresponds that eNOS would be preferentially targeted to signaling microdomains such as the MEJ. This allows for interaction with distinct protein partners that can regulate divergent physiological functions.

Basal eNOS phosphorylation occurs at both the apical EC and at the MEJ. ${ }^{11}$ In bovine EC, eNOS can be regulated by dual phosphorylation at the S635 and S1179 residues ${ }^{207}$, which indicates there may be other modifications of serine or threonine sites on MEJ eNOS after PE, as we solely focused on S1177 phosphorylation. Fleming et al has shown bradykinin stimulation of the EC resulted in rapid increases of S1177 phosphorylation and T495 dephosphorylation. These particular events were not influenced by the addition of PKC inhibitors but instead were dependent on the activity of calcium/calmodulin kinase II. ${ }^{208}$ Presumably, this process is occurring in the apical $\mathrm{EC}$ as the MEJ eNOS does not increase phosphorylation in response to bradykinin and is regulated in part by PKC. 
While PKC can be distributed throughout the cell, it has the ability to localize into subcellular signaling microdomains. ${ }^{209,210}$ Only one paper has noted the presence of any PKC isoform at the MEJ, and PKC itself was not the focus of that paper. ${ }^{12}$ There is precedent for PKC regulation of eNOS phosphorylation ${ }^{211-214}$ and the phosphorylation of eNOS being dependent on ER-derived calcium. ${ }^{215}$ The MEJ lipidomic results presented here point to, for the first time, 1) an ideal lipid environment for PKC based on the enrichment of PS and DAG and 2) a unique lipid environment in this arterial model as compared to EC and SMC grown in alone and in non-polarized conditions.

Using the pan-PKC inhibitor, Gö-6983, we observe a significant reduction in S1177 eNOS phosphorylation at the MEJ. It has been reported that overexpression of PKC $\alpha$ in EC results in increased eNOS phosphorylation at $1179^{212}$ and as both proteins are highly expressed at the MEJ, PKC $\alpha$ could contribute to eNOS phosphorylation at the MEJ. It is also possible the PKC $\alpha$ may act upstream of Akt, a kinase that has been shown to directly phosphorylate eNOS; studies have shown that PKC $\alpha$ activity influences AKT phosphorylation and activity. ${ }^{212,216,217}$ Regardless, the enrichment of PS and DAG provide the key substrates for PKC $\alpha$-AKT regulated eNOS phosphorylation providing another level of localized and controlled NO release to regulate specific functions in the vessel wall.

The lipid composition of cellular membranes can facilitate both the curvature of cellular projections (i.e. the MEJ) and cellular signaling via bioactive lipids or assembly of proteins and receptors important for signaling. Cholesterol content has never been investigated at the MEJ, and we found that it was significantly lower than EC cholesterol. The cell exhibits regional differences in cholesterol expression ${ }^{218}$ and cholesterol 
provides rigidity to membranes by packing tightly with saturated sphingolipids ${ }^{219}$, which would be an impediment to the dynamic extension and retraction that the MEJ exhibits ${ }^{1}$. Intracellular membranes, such as the one that belongs to the ER, have very low amounts of cholesterol and the cholesterol generated in the ER is exported rapidly to other organelles ${ }^{105}$. Therefore, the enrichment of ER at the MEJ could "dilute" the concentration of cholesterol that we measured. Another plausible reason may be to enhance NO diffusion to SMC-localized soluble guanylate cyclase; increased amounts of cholesterol inhibit NO diffusion through membranes. ${ }^{220}$

This study focuses on in vitro model of the MEJ, a system that reliably reproduces many characteristics of the MEJ that are seen in vivo. Investigating the MEJ in isolation via an intact artery is impossible in this context; it is not currently feasible to isolate an in vivo MEJ from the rest of the endothelium. While the entirety of this study was conducted in human primary cells, we postulate that the results presented here can be applicable to an intact physiological system. In summary, we have shown PE stimulation of SMC leads to MEJ specific eNOS phosphorylation while BK stimulation of EC only affects the apical pool of eNOS. These distinct pools exhibit differential effects of NO on the EC or SMC, owing to the unique lipid composition of the MEJ, localized ER and PKC activity. There is a strong possibility that the eNOS sequestration and selective activation in other polarized cell systems due to lipid membrane composition could also give rise to localized NO generation and function. 
CHAPTER 6. CALRETICULIN LOCALIZED TO THE MYOENDOTHELIAL JUNCTION MEDIATES HETEROCELLULAR SIGNALING AND VASCULAR FUNCTION 


\subsection{Abstract}

In resistance arteries, EC extensions can make contact with SMC, forming MEJ at holes in the internal elastic lamina (IEL). At these holes in the IEL, calcium is tightly regulated. Because calreticulin can buffer $\sim 50 \%$ of endoplasmic reticulum calcium and is expressed throughout holes in the internal elastic lamina of arteries, the only place where MEJs form, we investigated the effect of EC-specific calreticulin deletion on calcium signaling and vascular function. We found calreticulin expressed in nearly every IEL hole in third-order mesenteric arteries, but not other ER markers. Because of this, we generated an EC specific, tamoxifen inducible, calreticulin knockout mouse (EC Calr $\Delta / \Delta$ ). Using this mouse, we tested third-order mesenteric arteries for changes in calcium events at holes in the IEL and vascular reactivity after application of carbachol $(\mathrm{CCh})$ or phenylephrine (PE). We found that arteries from EC Calr $\Delta / \Delta$ mice stimulated with $\mathrm{CCh}$ had unchanged activity of calcium signals and vasodilation; however, the same arteries were unable to increase calcium events at the holes in the IEL in response to PE. This resulted in significantly increased vasoconstriction to PE, presumably due to inhibited negative feedback. In line with these observations, the EC Calr $\Delta / \Delta$ had increased blood pressure. Comparison of ER calcium in arteries and use of an ER-specific GCaMP indicator in vitro revealed no observable difference in ER calcium with Calr knockout. For this reason, we checked whether the calreticulin we observed at the IEL was ER-based. Using selective detergent permeabilization of the artery and inhibition of calreticulin translocation, we determined that calreticulin at holes in the IEL was not ER-based. Our data suggests calreticulin at holes in the IEL may act in a non-ER dependent manner to selectively regulate arteriolar heterocellular communication and blood pressure. 


\subsection{Introduction}

Maintenance of normal blood pressure relies on a balance of small diameter artery constriction and dilation which contributes to peripheral vascular resistance. ${ }^{221}$ Receptormediated signaling that occurs within endothelial cells (ECs) and smooth muscle cells (SMCs) in the vascular wall is particularly key to vascular tone regulation. ${ }^{73,222}$ Any perturbation in these signaling processes in resistance arteries can shift blood pressure away from homeostasis and towards hypertension. ${ }^{223,224}$

Calcium is a tightly regulated signaling molecule in the vascular wall with cytosolic increases having opposing effects on vascular tone. ${ }^{8}$ Unstimulated cells maintain cytosolic calcium at nanomolar levels, thus increases in EC cytosolic calcium result in vasodilation; increased SMC cytosolic calcium results in vasoconstriction. However, increases in SMC calcium and inositol 1,4,5 trisphosphate $\left(\mathrm{IP}_{3}\right)$ via alpha1D adrenergic receptor stimulation with phenylephrine have been shown to elicit subsequent increases in EC calcium ${ }^{23,24,156}$, indicating heterocellular communication occurs between the two cell types. In resistance arteries, the localized EC calcium after phenylephrine stimulation occurs at EC and SMC sites of cellular contact (myoendothelial junctions (MEJ)) through the internal elastic lamina (IEL). ${ }^{3,} 225$ The prevalence of MEJs increases with decreasing vessel size ${ }^{226}$, underscoring its strategic location in arteries that mediate vascular tone and blood pressure.

Because the MEJ is a projection originating from the EC, increased intracellular calcium at the MEJ is important for arterial relaxation and negative feedback after arterial constriction..$^{9}$ 13, 14, 16, 18, 19, 23, 227 In particular, endoplasmic reticulum (ER) -based calcium signals have been thought to arise from $\mathrm{IP}_{3}$ receptors on the ER that surrounds MEJs. ${ }^{13,14}$, 18, 19, 26 Extracellular influx of calcium via transient receptor potential vanilloid 4 (TRPV4) 
channels has been shown to regulate extracellular influx of calcium into the MEJ and vascular tone. ${ }^{9,16}$ However, the role of ER calcium at the MEJ on vascular tone and signaling is still unclear, due mostly to being unable to manipulate ER-based proteins in vivo.

Calreticulin (Calr), a protein well-known for sequestering ER calcium ${ }^{150,228}$, was shown by our lab to be highly expressed at the MEJ in vitro. ${ }^{10,38}$ In experiments focused on Calr cell biology, the knockdown or overexpression of Calr has profound effects on ER calcium; deletion results in reduced ER calcium and overexpression increases ER calcium. ${ }^{60,61,229}$ Furthermore, Calr deficient cells have impairments in $\mathrm{IP}_{3}$ stimulated calcium mobilization..$^{42,52,230}$ Until now, the role of Calr in the context of vascular tone has not been investigated. In this study, we used a novel mouse model specific to endothelium and induced knockout after development in order to avoid issues with Calr and organogenesis. ${ }^{52,53,55}$ Given the effects of Calr on ER calcium and ER calcium release, we predicted we would see similar impairments in IP 3 mediated signaling and hypothesized this would result in changes in calcium signaling, vascular tone and blood pressure.

Surprisingly, we found that MEJ localized Calr is unique compared to the EC monolayer and that EC deletion of Calr results in a polarized response to IP 3 stimulation depending on receptor activation. This alteration in signaling affects vascular tone and blood pressure. Importantly, we show this polarization of response is likely not due to differences in ER calcium content, and may actually be due to MEJ Calr localization outside the ER. 


\subsection{Results}

Calreticulin is enriched at holes in the internal elastic lamina

We investigated the expression of various ER localized proteins in the context of expression at holes in the internal elastic lamina (HIEL). Calnexin (Figure 12A) and ERp29 (Figure 12B) were throughout the EC monolayer (Figure 12G), but less so ( 30\%) within HIEL, the only place where MEJ form. However, Calr was present in EC monolayers and nearly all the HIEL (Figure 12C-D). The secondary antibody alone and IgG negative controls could not detect any positive staining (Figure 12E-F). Quantification of positive Calr staining was found in $>80 \%$ of the HIEL (Figure $12 \mathrm{H}$ ). We used immuno-electron microscopy and confirmed Calr expression in ECs and MEJs (Figure 13).

Generation of tamoxifen inducible, endothelial specific, Calr knockout mouse

Because Calr is expressed in ECs and within MEJs, and ER calcium could be an important regulator of vascular function, we hypothesized that EC deletion of Calr could mediate heterocellular calcium signaling. For these reasons, we made a tamoxifen inducible, EC-specific Calr knockout mouse (EC Calr $\Delta / \Delta$; Figure 14A). To check the efficiency of Calr knockout, we used primers designed to detect the truncated Calr gene, with a size of approximately $400 \mathrm{bp}$ only seen in EC Calr $\Delta / \Delta$ mice $^{55}$ (Figure 14B). Third order mesenteric arteries from these mice were stained for Calr (Figure 14C) and a significant reduction in Calr at HIEL was identified in EC Calr $\Delta / \Delta$ mice (Figure 14D). To more specifically look at EC Calr expression, we isolated microvascular diaphragm ECs and found EC Calr $\Delta / \Delta$ mice had significantly fewer ECs (CD31+ cells) with Calr (Figure 14, 15). There were no differences in the density of HIEL (Figure 14F) or blood cell counts (Figure 15) between mice. Mesenteric arteries were also sectioned and stained with 
hematoxylin and eosin or trichrome to visualize collagen and there were no obvious differences (Figure 17). However, the investigation of binding immunoglobulin protein (BiP) expression showed a significant increase in aortas from EC Calr $\Delta / \Delta$ mice and in primary human aortic EC transfected with Calr siRNA (Figure 19).

\section{Calcium events in EC monolayer and HIEL of EC Calr NAarteries}

To investigate the physiological effect of EC Calr knockout, a muscarinic agonist (carbachol; CCh) and an adrenergic agonist (phenylephrine; PE) were used to selectively target calcium events at HIEL (Figure 20A). Carbachol stimulation caused a significant increase in calcium events in the EC monolayer in both EC Calr fl/fl and $\Delta / \Delta$ mice (Figure 20B). Phenylephrine application had no effect on calcium events in the EC monolayer (Figure 20B), underscoring its selective site of action in the HIEL.

When we focused on calcium events occurring in the endothelium specifically at HIEL (Figure 20C-D), CCh significantly increased calcium events at HIEL in both the EC Calr fl/fl and EC Calr $\Delta / \Delta$ arteries (Figure 20E-F). Addition of PE to the arteries caused a significant increase in EC Calr fl/fl calcium events, however EC Calr $\Delta / \Delta$ arteries did not exhibit this same increase (Figure 20G-H). Thus, EC Calr $\Delta / \Delta$ arteries are able to significantly increase calcium events in response to $\mathrm{CCh}$, but not $\mathrm{PE}$, and these differences occur at the HIEL.

\section{Constriction and dilation of EC Calr $\Delta \Delta$ arteries}

Because localized calcium events in the HIEL can affect vascular tone ${ }^{9,16}$, the effect of EC Calr $\Delta / \Delta$ on dilation to $\mathrm{CCh}$ and constriction to PE was investigated. In line with our calcium data, dilation to $\mathrm{CCh}$ over a range of concentrations was not different between groups (Figure 21, 22A-B). However, EC Calr $\Delta / \Delta$ arteries constricted significantly more 
to PE than control (Figure 22C-D), functional evidence that coincides with the observed polarized calcium events and in-line with reduced negative feedback. However, spontaneous myogenic tone at $80 \mathrm{mmHg}$ (Figure 22E) was not altered, indicating EC Calr deletion does not affect intrinsic properties of SMC. Similarly, NS309-induced dilation did not differ between groups (Figure 22F). Sodium nitroprusside induced dilation and $\mathrm{KCl}$ induced constriction were not different (Figure 26).

Given the calcium imaging and vascular reactivity results, we next investigated whole animal mean arterial pressure (MAP) via radiotelemetry catheters. Before tamoxifen injection, MAP was not different (Figure 22G, solid bars) but after induction of EC Calr deletion, EC Calr $\Delta / \Delta$ mice significantly increased their MAP relative to each mouse's own pre-injection baseline (Figure 22H) with an unaffected heart rate (Figure 27). This increase occurred several weeks after tamoxifen induced deletion of Calr.

Separate experiments looking at acute changes in blood pressure showed no difference in blood pressure 2-3 weeks after tamoxifen injections (Figure 23). Injection of $0.1 \mathrm{mg} / \mathrm{kg}$ PE did significantly increase systolic blood pressure within the first 15 minutes, but there was not a difference in the response between groups (Figure 24A). EC Calr $\Delta / \Delta$ mice had a significantly blunted diastolic blood pressure response to PE (Figure 24B).

Furthermore, mice were implanted with radiotelemetry catheters and osmotic pumps containing angiotensin II (Ang II). There was no significant difference between the blood pressure response to Ang II over the 14 days of infusion (Figure 25).

\section{Calreticulin does not affect the level of endoplasmic reticulum calcium}

In order to determine if the EC Calr deletion was altering the ER calcium, we first incubated arteries with $0 \mathrm{mM}$ extracellular calcium (to prevent extracellular calcium from 
entering the cell) followed by CPA (to block SERCA mediated refilling of the ER). Peak cytosolic calcium, specifically released from the ER, did not show any difference in ER calcium content in the arteries (Figure 28A). Next, primary human EC were transfected with GCaMP-ER to specifically visualize ER calcium bound to the low affinity indicator (Figure 28, 29, 30). Co-transfection of human EC with Calr siRNA for 48 hours resulted in a $\sim 40 \%$ decrease in Calr protein as measured by western blot (Figure 28B, 31) which was concordant with our whole animal knockdown (Figure 14). GCaMP-ER fluorescence was measured via flow cytometry in live EC and divided into low, medium and high fluorescence intensity (Figure 28C). There was no difference in the amount of fluorescence between GCaMP alone, GCaMP co-transfected with Calr siRNA or GCaMP co-transfected with scrambled siRNA (Figure 28D, 29). This data coincides with the intact arteries indicating the knockdown of Calr did not alter free ER $\mathrm{Ca}^{2+}$ levels.

\section{Subcellular localization of Calr in the MEJ}

Due to the lack of changes in total ER calcium content, we further hypothesized that the Calr at the HIEL and MEJ may be regulating ER calcium signals independently of its calcium buffer function within the ER. Using digitonin to selectively permeabilize the plasma membrane (but not $\mathrm{ER}^{231,232}$ ), we repeated the en face immunofluorescence for calnexin (Figure 34A), ERp29 (Figure 34B) and calreticulin (Figure 34C) in wild-type mouse mesenteric arteries. In both the EC monolayer and the HIEL, there was no signal for calnexin and ERp29 (Figure 34D-E). The Calr staining exhibited strong punctate signal in the HIEL (Figure 34E). In contrast to the rigorous permeabilization of Triton-X100 which permeabilizes all membranes, digitonin, which preferentially permeabilizes the plasma membrane and not the ER membrane, resulted in no observable staining in the EC 
monolayer (Figure 34D) with any of the ER-markers, including Calr. Next, we attempted to inhibit Calr expression at HIEL. Because Calr in the cytosol is derived from the ER 233 and could be trafficked out of the $\mathrm{ER}^{48}$ we incubated arteries with Brefeldin A, which inhibits anterograde traffic out of the ER. This treatment caused a significant decrease in the Calr fluorescence in HIEL, but not the EC monolayer (Figure 34F-H). Pressure myography on mesenteric arteries incubated with Brefeldin A tended to decrease constriction to PE (Figure 33), but this may be due to effects on SMC receptor trafficking to the membrane.

Further evidence of Calr polarization of function was found from immunoprecipitated calreticuin, which demonstrated selective binding to BiP in EC monolayers (an association commonly found in the ER), and binding to thrombospondin1 in the MEJ fractions (an association commonly found outside of the ER; Figure 32). This data suggests the Calr in endothelial monolayers of arteries is found in the ER, but the Calr at HIEL is not ER-based.

The effect of aging on calcium signaling in EC Calr fl/fl and $\mathrm{N \Delta}$

Aging is associated with impairments in endothelial function which leads to decreased endothelial dependent dilation and increased blood pressure. ${ }^{234}$ The structure of small and large arteries changes; collagen increases in the extracellular matrix, which leads to less elastic arteries and further contributes to age-associated hypertension. ${ }^{234}$ In 24-26 month old mice, the number of holes in the IEL decrease and the diameters of these holes are smaller. ${ }^{227}$ Calcium signaling in the vascular wall is detrimentally affected with advanced age and it is likely changes in calcium are related to changes in arterial structure. In aged mesenteric IEL holes, the sites of spontaneous basal calcium 
release decrease, the frequency of events decrease and the amplitudes of these events increase. ${ }^{227}$ Because these spontaneous MEJ events are mediated primarily by IP3R, dysregulation of ER calcium release with age is a likely mechanism.

Using the EC Calr fl/fl and EC Calr $\Delta / \Delta$ third order mesenteric arteries and specifically focused on events occurring within the IEL holes, we characterized the calcium events that occurred at baseline and in response to $\mathrm{CCh}$ and PE in mice aged at least 75 weeks (Figure 36). Aged arteries were also compared to the young arteries $(<20$ weeks old) used for calcium events in Chapter 6 (Figure 35). We also investigated the expression of Calr mRNA in thoracic aortas of young and aged mice and found Calr in the vascular wall decreases with age (Figure 35).

Aged EC Calr fl/fl mice are still able to generate significant increases in calcium events in response to muscarinic and adrenergic stimulation (Figure 36). Aged EC Calr $\Delta / \Delta$ arteries are able to increase calcium events in response to $\mathrm{CCh}$, but there are fewer number of events and a threefold difference when compared to their aged controls (Figure $36 \mathrm{~A}, \mathrm{~B}$ ). Aging in EC Calr fl/fl does not change in the number of events at baseline or with receptor stimulation (Figure 36). However, Calr expression in thoracic aortas was decreased with age in these mice (Figure 35A).

\section{Peptide generation}

En face preparations of third-order arterioles demonstrated an abundance of ITPKA in holes of the IEL (Figure 37A, B). Thus, there is a unique localization of (possibly non-ER based) Calr and ITPKA at MEJs in vitro and in vivo. Because of the evidence for interaction of Calr and ITPKA, we performed in silico analysis. We used Protein Data Bank (PDB) structures to generate a model of Calr and IPKA interaction and developed an 
ITPKA mimetic peptide to disrupt this binding (Figure 37C). Using the peptide, we found significantly less constriction to PE versus the scrambled peptide (Figure 37D). However, this peptide did not significantly alter CCh dilation (Figure 37E).

A model was generated based on Kinesin family member 18A (Kif18A, similar sequence homology to Kif18b) and Calr binding and a peptide was generated to disrupt this interaction (Figure 38A). Phenylephrine dose response in isolated arteries did not show any differences in constriction between the Kif18B and scrambled peptides (Figure 38B). 
FIGURE 12. CALRETICULIN (CALR) IS ENRICHED AT THE MAJORITY OF HOLES IN THE ARTERIAL ELASTIC LAMINA
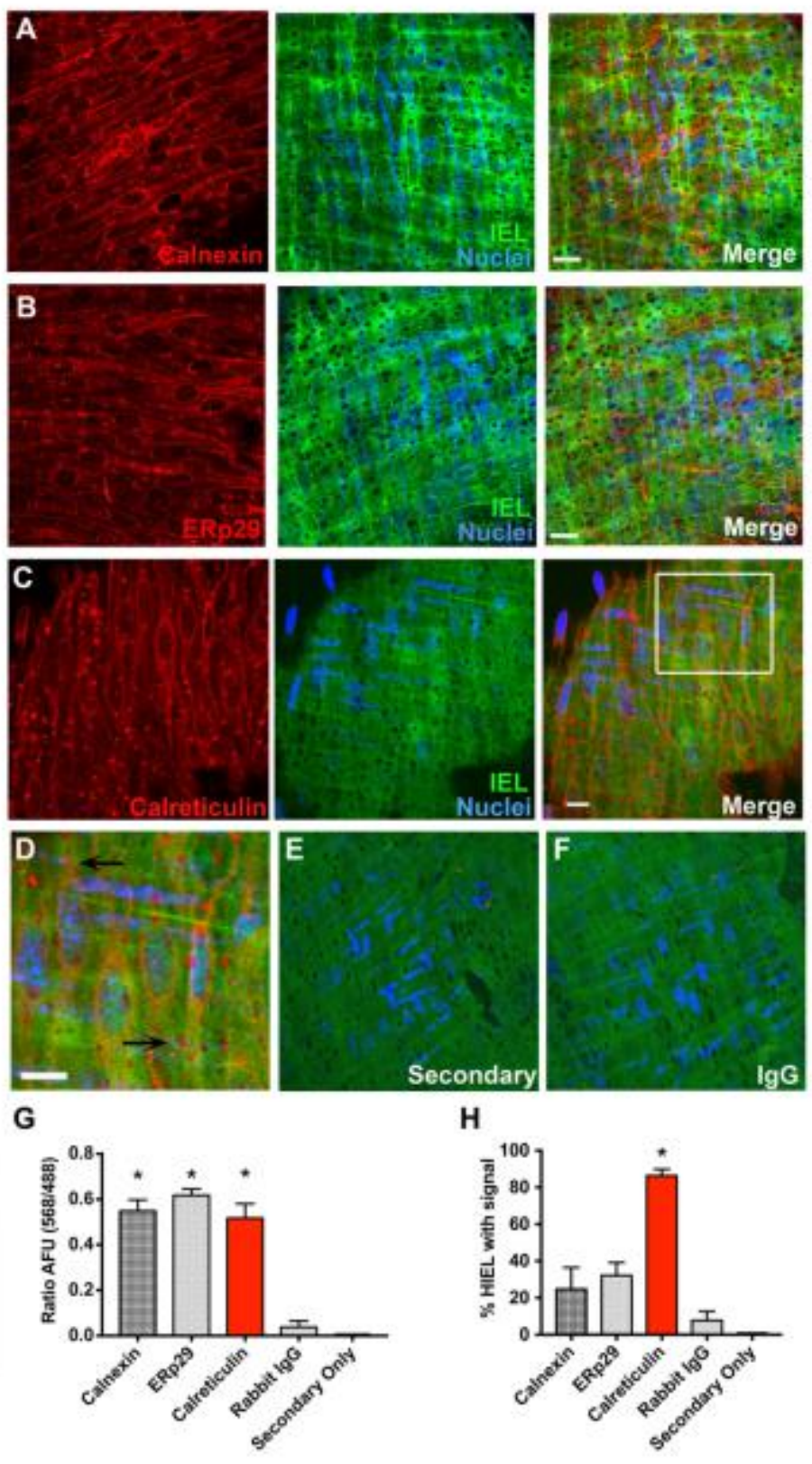
Figure 12. Calreticulin is enriched in the majority of holes in the arteriole internal elastic lamina (IEL). Confocal Z-stack of en face immunofluorescence for established endoplasmic reticulum (ER) markers, including A calnexin (red), B ERp29 (red), or C calreticulin (Calr; red) in a third order mesenteric arteriole with autofluorescence of the internal elastic lamina (IEL; green) and DAPI stained nuclei from endothelial cell (EC; blue). Merge indicates overlay of the three fluorescent channels. D, Magnified region from $\mathrm{C}$ indicating positive signal in the perinuclear region of the EC and holes of the IEL (HIEL; black arrows). E, Separate experiments show a representative arteriole incubated with secondary antibody only or $\mathbf{F}$ rabbit IgG instead of primary antibody. G, Quantification of EC monolayer fluorescence $(568 \mathrm{~nm})$ normalized to IEL autofluorescence $(488 \mathrm{~nm})$ in arbitrary fluorescent units (AFU). * $\mathrm{p}<0.05$ versus rabbit $\operatorname{IgG}$ and secondary only. $\mathbf{H}$, Percentage of HIEL with positive staining. * $\mathrm{p}<0.05$ versus calnexin, ERp29, rabbit IgG and secondary only . Calreticulin/Secondary only/Rabbit $\operatorname{IgG}, \mathrm{n}=6$ fields of view. ERp29/calnexin, $\mathrm{n}=3$ fields of view. Scale bar $=10 \mu \mathrm{m}$. 
FIGURE 13. QUANTIFICATION OF CALR IN EC AND MEJ VIA IMMUNOTRANSMISSION ELECTRON MICROSCOPY

A
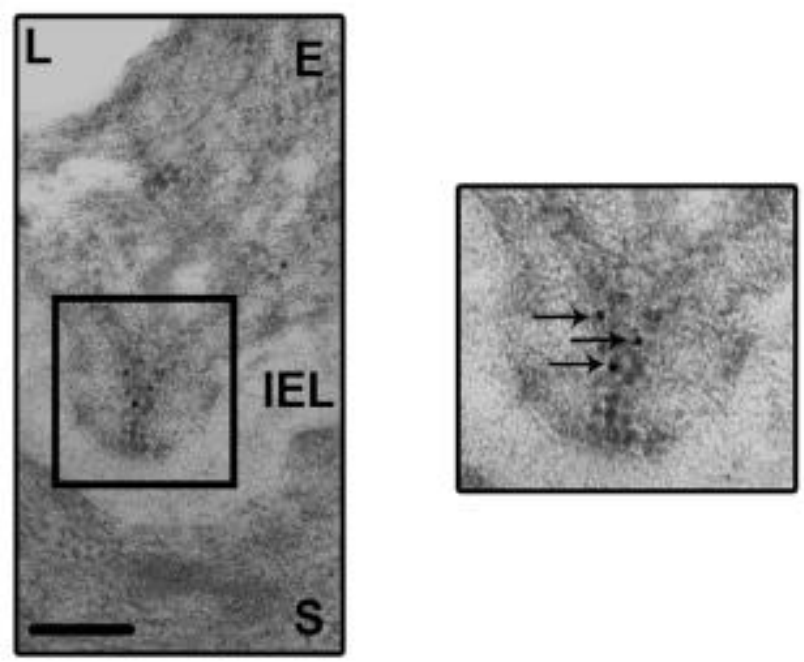

B

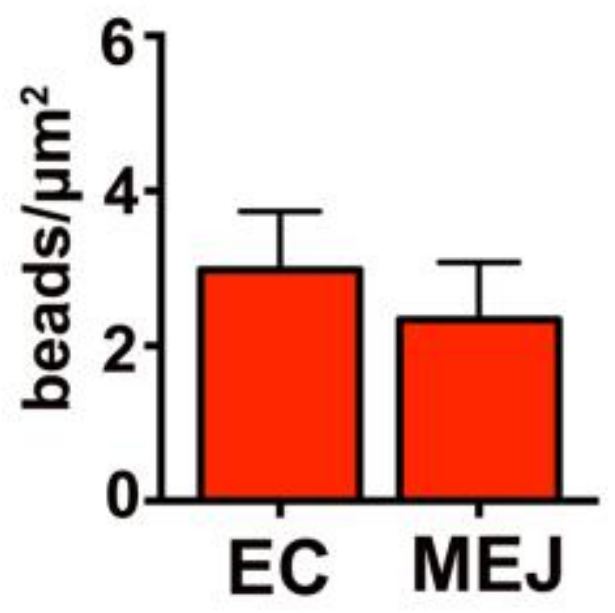


Figure 13. Quantification of Calr in the EC and MEJ via immuno-transmission electron microscopy. A, Representative electron micrograph image of transverse section of thoracodorsal artery showing lumen (L), endothelium (E), smooth muscle (S) and MEJ (outlined with black box) projecting through the internal elastic lamina (IEL). The magnified region indicates gold bead staining for calreticulin at the MEJ (black arrows). Scale bar $=0.5 \mu \mathrm{m} \mathbf{B}$, Quantification of gold beads per $\mu \mathrm{m}^{2}$ in the EC monolayer and MEJ. ( $\mathrm{n}=3$ arterioles from $3 \mathrm{C} 5 \mathrm{~B} 1 / 6$ mice, average of 6.33 pictures per arteriole) 
FIGURE 14. GENERATION OF EC SPECIFIC, TAMOXIFEN INDUCIBLE CALR KNOCKOUT MOUSE

A

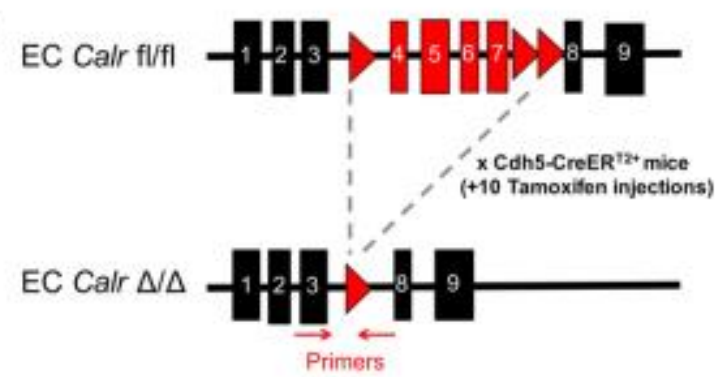

B

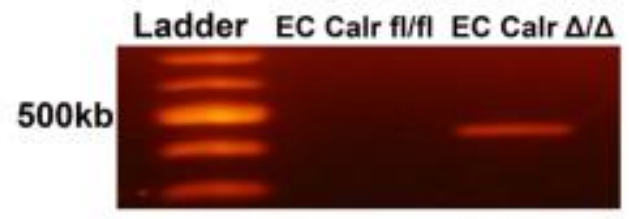

C
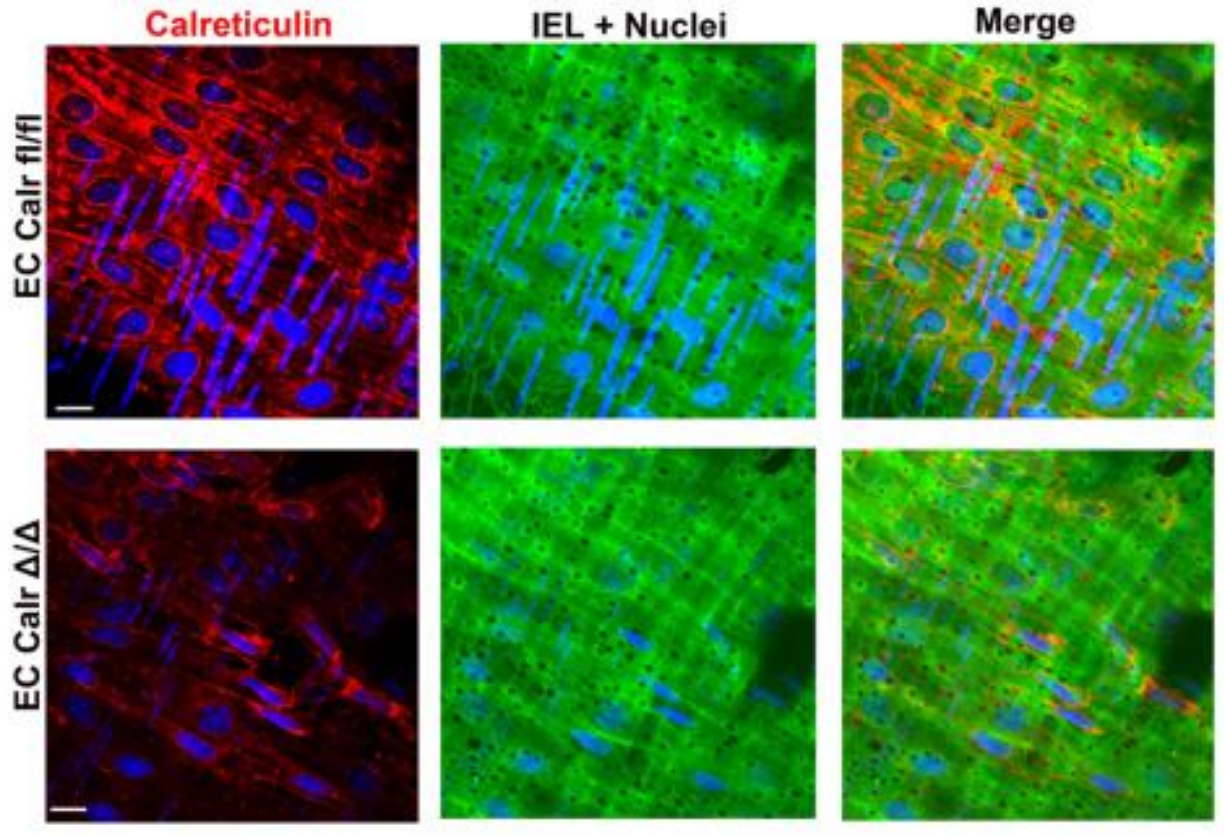

D

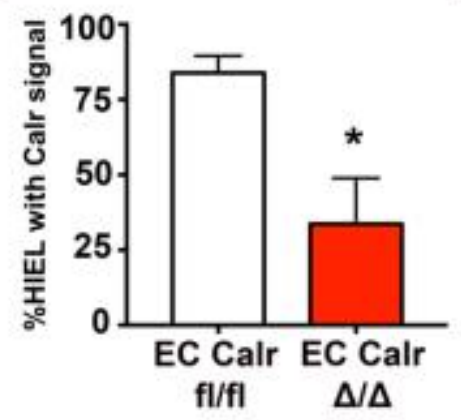

E

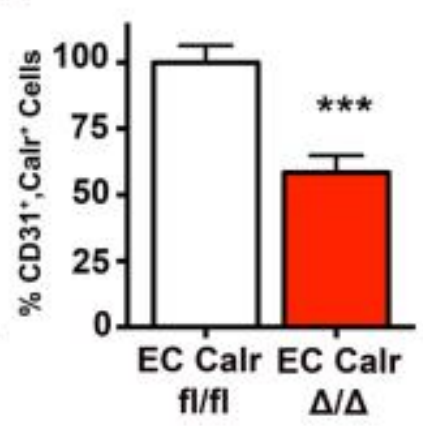

$\mathbf{F}$

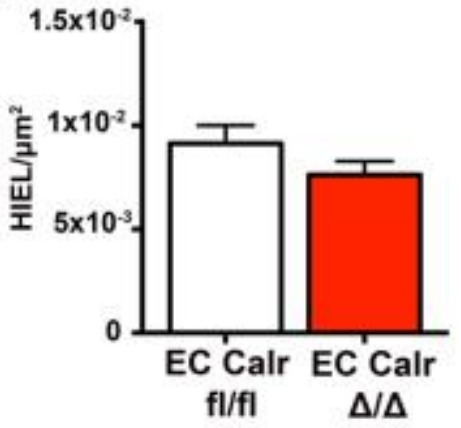


Figure 14. Generation of EC specific, tamoxifen inducible Calr knockout mice. A, Gene map showing loxP sites (red triangles) around exons 4-7 in the Calr gene. The Calr floxed mice were bred with EC specific promoter mice $\left(\mathrm{Cdh} 5-\mathrm{CreER}^{\mathrm{T} 2+}\right)$. All EC Calr fl/fl mice were injected for 10 days with vehicle control (peanut oil, EC Calr fl/fl) or tamoxifen (EC Calr $\Delta / \Delta$ ). Red arrows in $\mathbf{A}$ indicate location of primers for $\mathbf{B}$, Representative endpoint PCR gel indicating excision of Calr exons only in EC Calr $\Delta / \Delta$ with a product of approximately 400 kilobase pairs (kb). C, Representative images of Calr (red) en face immunofluorescence in arterioles (green=autofluorescence of IEL, blue= nuclei). Scale bar $=10 \mu \mathrm{m}$. D, Percentage of HIEL with Calr signal in EC Calr fl/fl and $\Delta / \Delta$ arterioles (EC Calr fl/fl $\mathrm{n}=5$ fields of view; EC Calr $\Delta / \Delta \mathrm{n}=5$ fields of view). E, Diaphragm microvasculature was digested, stained for CD31 (EC marker) and Calr and analyzed via flow cytometry. (EC Calr fl/fl n=6, EC Calr $\Delta / \Delta \mathrm{n}=5$ ) $\mathbf{F}$, HIEL were quantified to approximate the number of myoendothelial junctions (MEJ). Images were from en face arterioles using autofluorescence of the IEL (EC Calr fl/fl $\mathrm{n}=22$ fields of view, EC Calr $\Delta / \Delta \mathrm{n}=19$ fields of view). $*$ indicates $\mathrm{p}<0.05, * * * \mathrm{p}<0.001$. 
FIGURE 15. REPRESENTATIVE FLOW CYTOMETRY PLOTS FOR EC CALR FL/FL AND EC CALR $\triangle / \triangle$ DIAPHRAGM AND CALR MEAN FLUORESCENCE INTENSITY

A

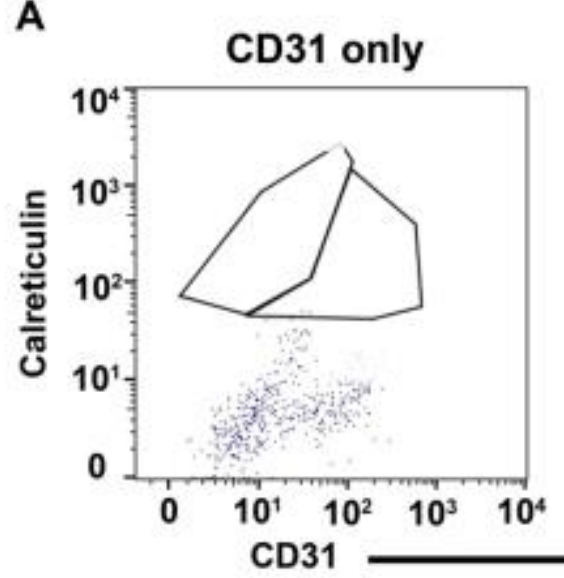

C

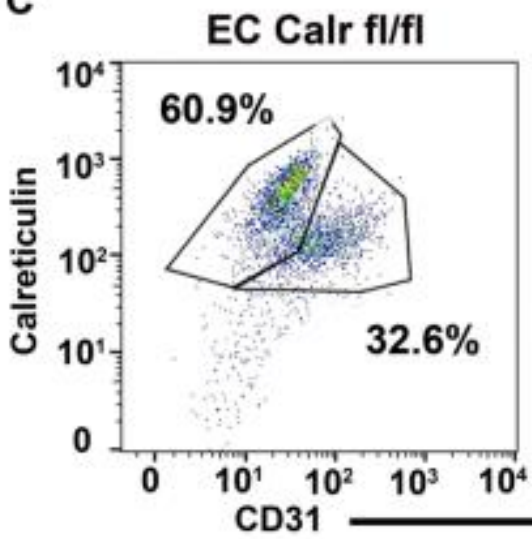

B Calr only

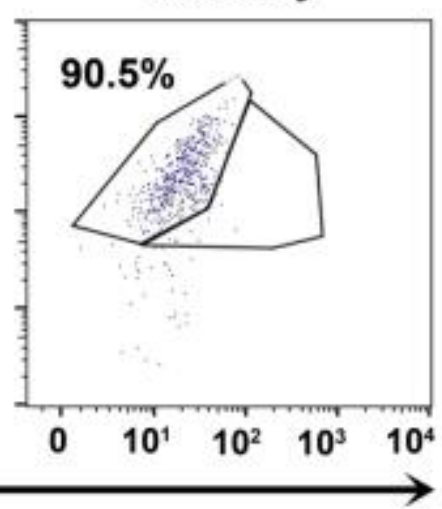

D
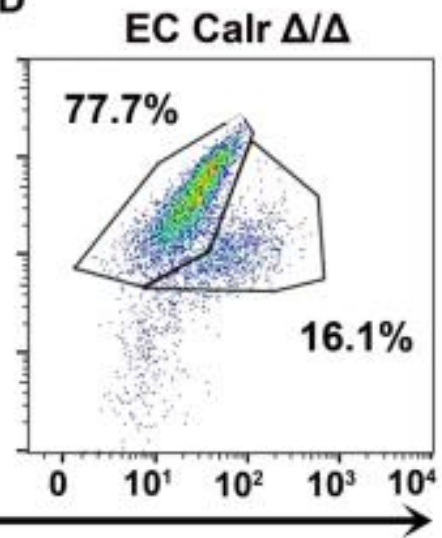

E

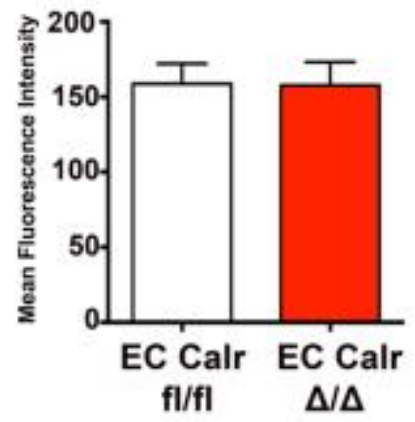


Figure 15. Representative flow cytometry plots for EC Calr fl/fl and EC Calr $\Delta / \Delta$ diaphragm and Calr mean fluorescence intensity. Diaphragms containing microvascular EC were incubated with fluorescent antibodies for $\mathbf{A}$, CD31 and $\mathbf{B}$, Calreticulin. Then, diaphragm EC from $\mathbf{C}, \mathrm{EC}$ Calr fl/fl and $\mathbf{D}$, EC Calr $\Delta / \Delta$ mice were analyzed for CD31 with expression of Calr. E, Mean fluorescent intensity was calculated for CD31+, Calr+ cells (EC Calr fl/fl $\mathrm{n}=6$, EC Calr $\Delta / \Delta \mathrm{n}=7$ ). 
FIGURE 16. EC CALR $\Delta / \Delta$ DOES NOT CHANGE BLOOD CELL INDICES

A

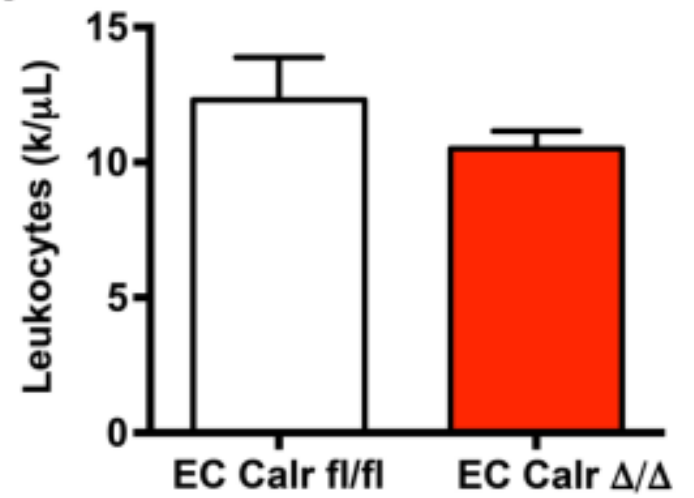

C

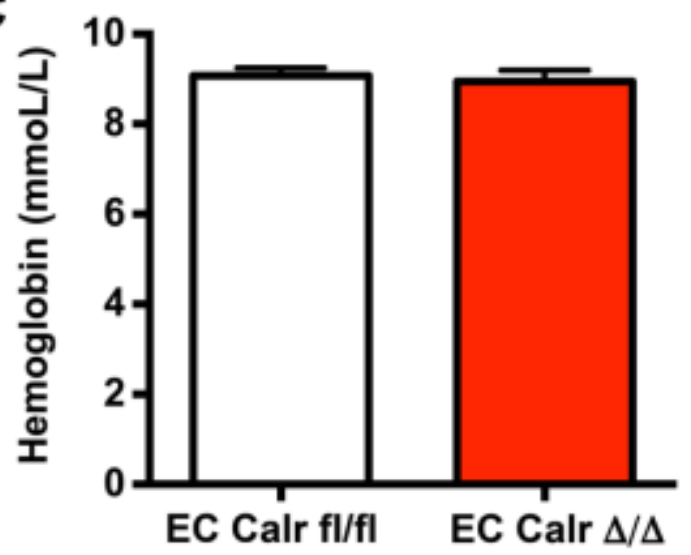

B

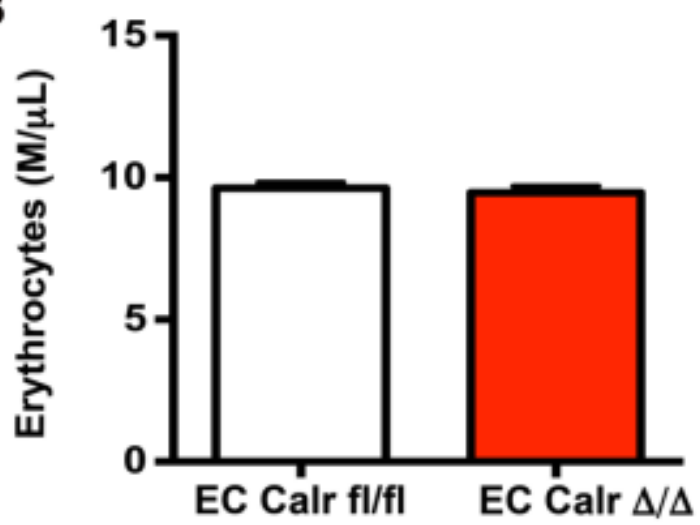

D

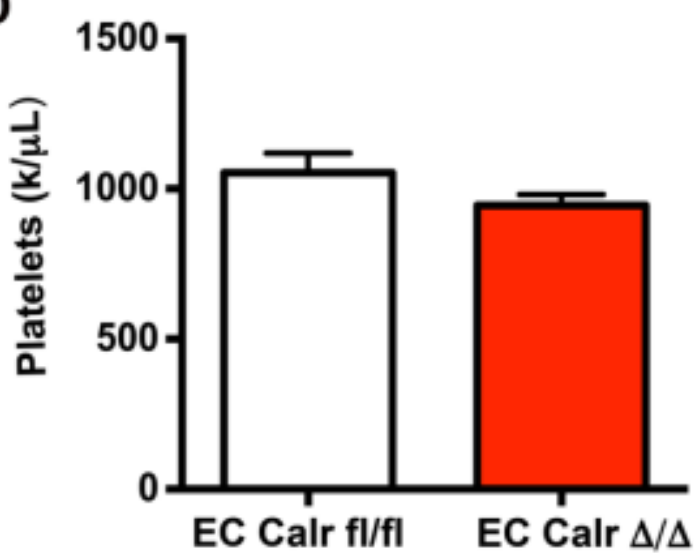

Figure 16. EC Calr $\Delta / \Delta$ does not change blood cell indices. Blood from the retro-orbital sinus was collected from lightly anesthetized EC Calr fl/fl and EC Calr $\Delta / \Delta$ via microhematocrit tubes and analyzed. Total $\mathbf{A}$, leukocytes $\mathbf{B}$, erythrocytes $\mathbf{C}$, hemoglobin and $\mathbf{D}$, platelets are not different between EC Calr fl/fl $(\mathrm{n}=5)$ and EC Calr $\Delta / \Delta$ mice $(\mathrm{n}=5)$. 
FIGURE 17. REPRESENTATIVE TRICHROME AND HEMATOXYLIN/EOSIN

STAINED ARTERIES FROM EC CALR FL/FL AND EC CALR $\Delta / \Delta$ MICE.

A

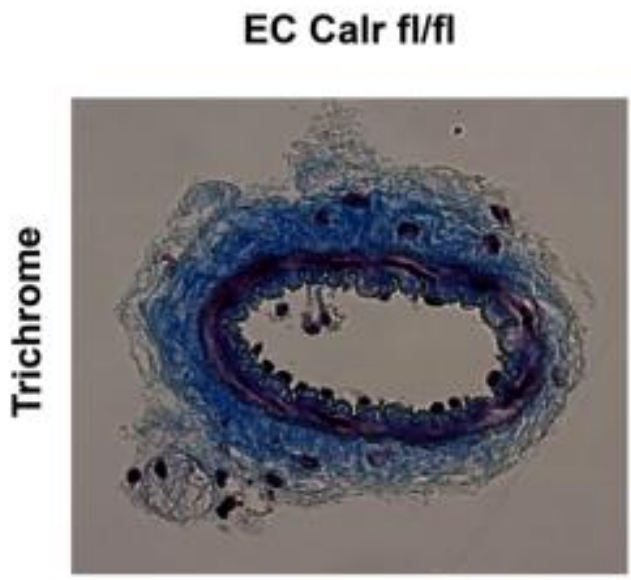

C

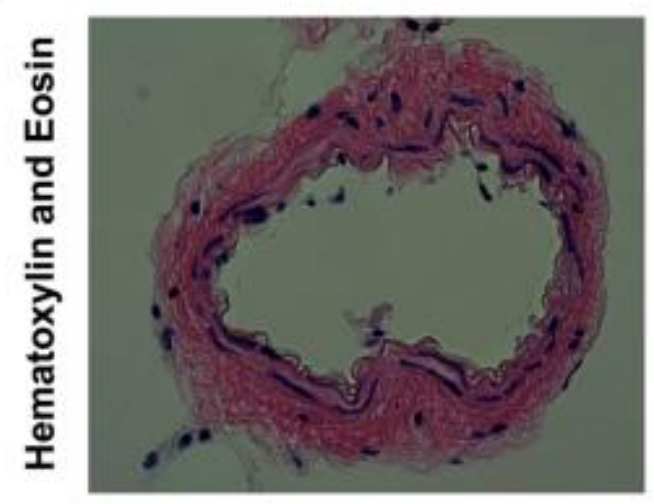

B EC Calr $\Delta / \Delta$

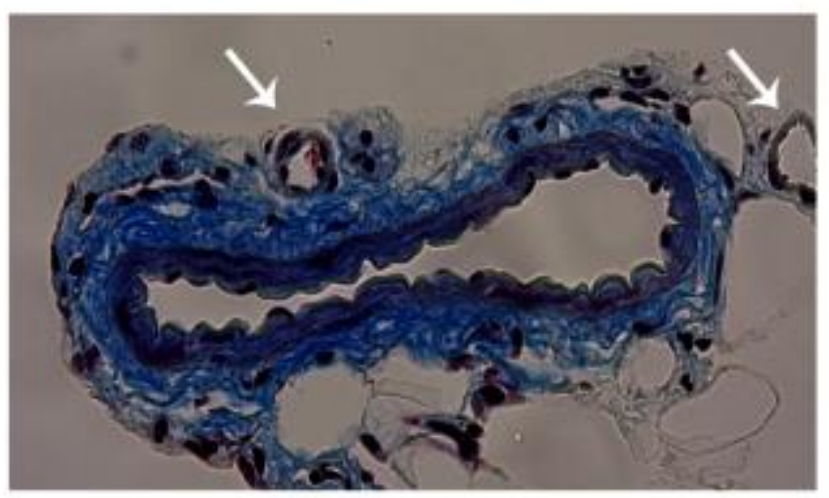

D

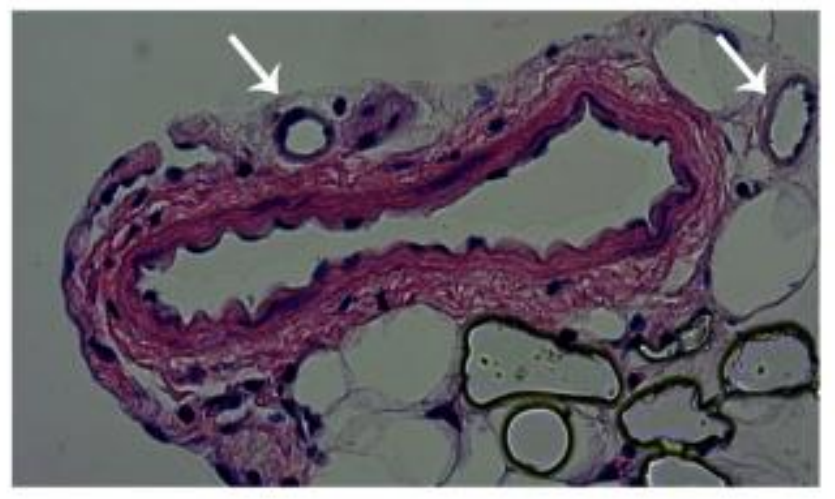

Figure 17. Representative trichrome and hematoxylin/eosin stained arteries from EC Calr fl/fl and EC Calr $\Delta / \Delta$ mice. A, B, Trichrome stain for collagen in third order mesenteric arteries. Blue indicates presence of collagen and black indicates nuclei. $\mathbf{C , ~ D ,}$ Hematoxylin and eosin stain for cytoplasm and nuclei, respectively. Arrows in B and D indicate possible small arteries in the adventitia of the vessel. 
FIGURE 18. RELATIVE EXPRESSION OF mRNA IN THORACIC AORTAS FROM EC CALR FL/FL AND EC CALR $\Delta / \Delta$ MICE.

A

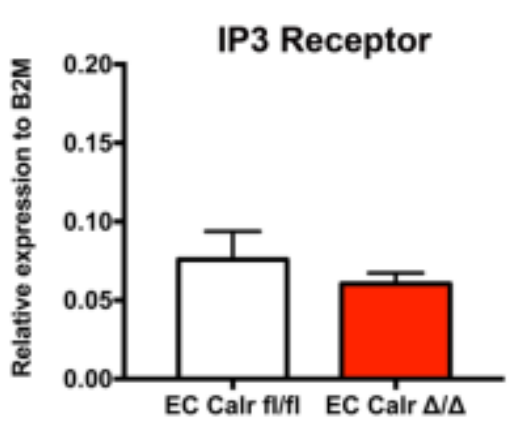

B

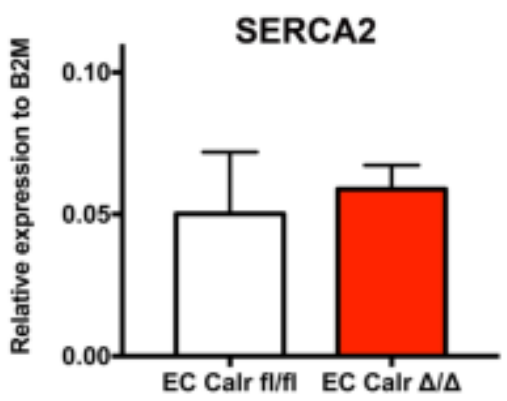

C

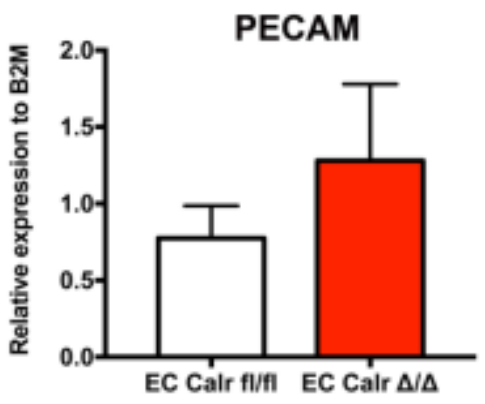

Figure 18. Relative expression of mRNA in thoracic aortas of EC Calr fl/fl and EC Calr $\Delta / \Delta$ mice. A, IP3 Receptor expression is not different (EC Calr fl/fl $\mathrm{n}=5$, EC Calr $\Delta / \Delta$ $\mathrm{n}=2$ ). B, Serca2 expression is not different (EC Calr fl/fl $\mathrm{n}=4$, EC Calr $\Delta / \Delta \mathrm{n}=3$ ). C, No significant difference is seen in Pecam mRNA (EC Calr fl/fl $\mathrm{n}=5$, EC Calr $\Delta / \Delta \mathrm{n}=3$ ). 


\section{FIGURE 19. BINDING IMMUNOGLOBULIN PROTEIN (BiP) EXPRESSION} AFTER EC CALR KNOCKOUT.

A

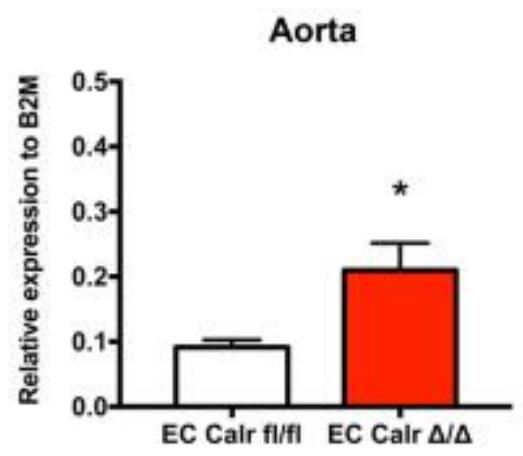

C

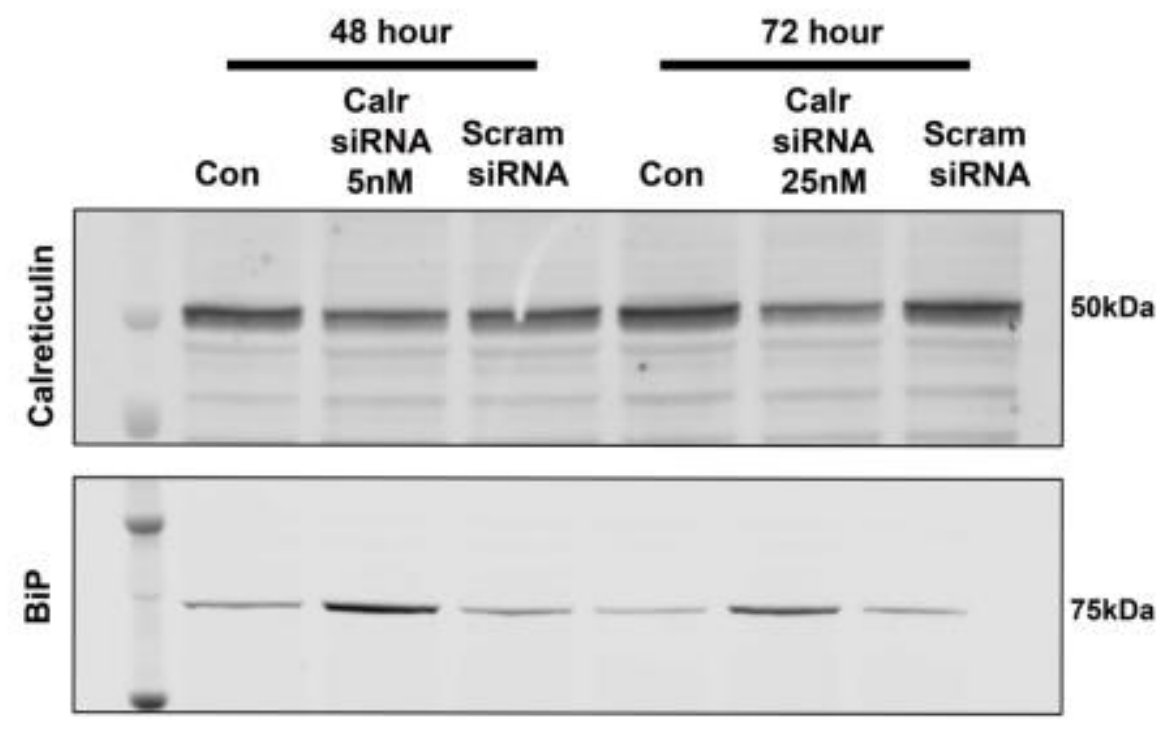

B

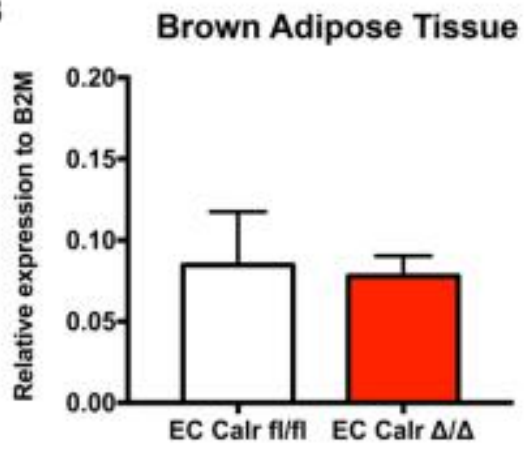

EC Calr filfl EC Calr $\Delta / \Delta$ 
FIGURE 20. EC CALR $\Delta / \Delta$ ARTERIES HAVE DIFFERENTIAL, IEL LOCALIZED CALCIUM RESPONSES TO ADRENERGIC VERSUS MUSCARINIC AGONISTS

A

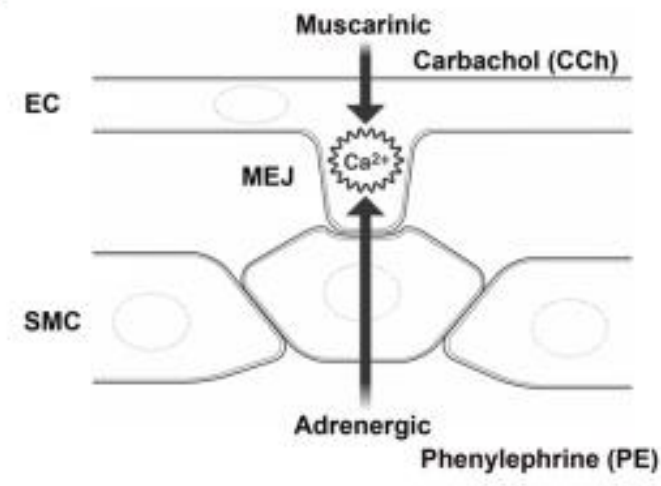

C

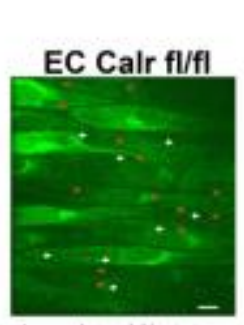

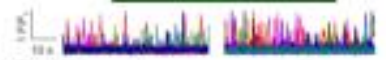

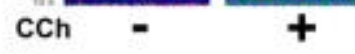

E

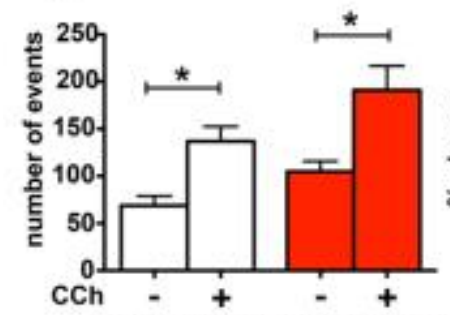

EC Cair fl/fl EC Calr $\Delta / \Delta$
$\mathrm{CCh}$

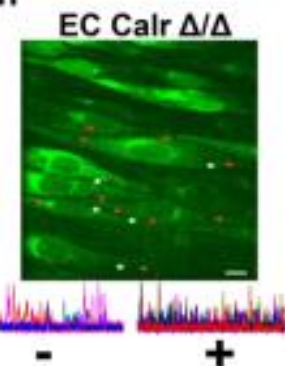

$\mathbf{F}$

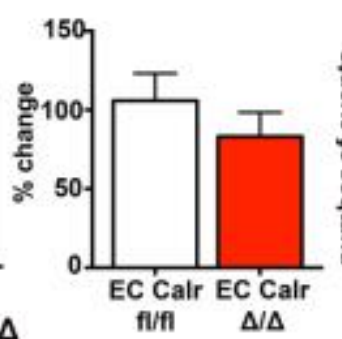

B

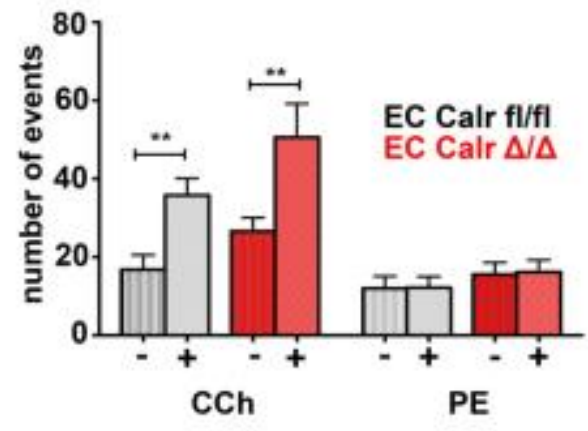

D
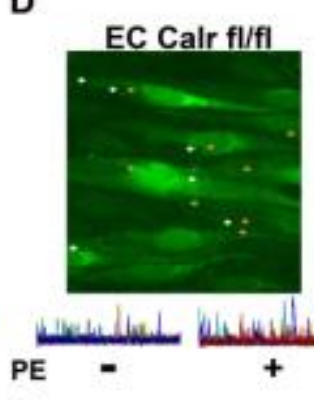

G

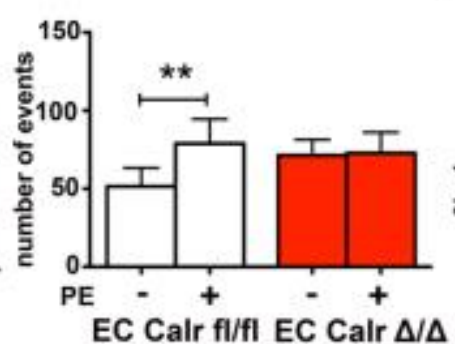

PE

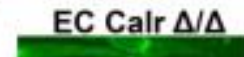

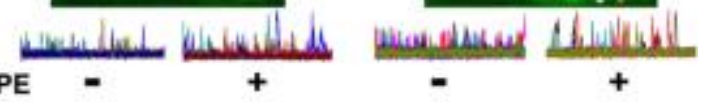

H

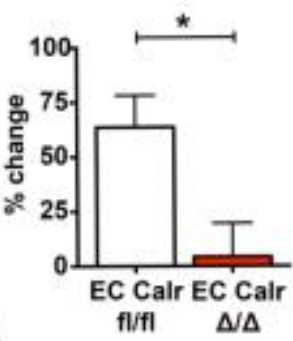


Figure 20. EC Calr $\Delta / \Delta$ arterioles have differential, IEL localized calcium responses to adrenergic versus muscarinic agonists. A, Schematic showing both muscarinic stimulation (via carbachol; $\mathrm{CCh}$ ) and adrenergic stimulation (via phenylephrine; $\mathrm{PE}$ ) cause increases in calcium events at the MEJ. En face preparations of third order mesenteric arteries were loaded with fluo-4AM, incubated in physiological salt solution and maintained at $37^{\circ} \mathrm{C} . \mathbf{B}$, Number of calcium events occurring in the endothelial (EC) monolayer (not within IEL holes) to CCh and PE. C, D Representative images show locations of baseline (white arrows) and stimulated (red or orange arrows) events

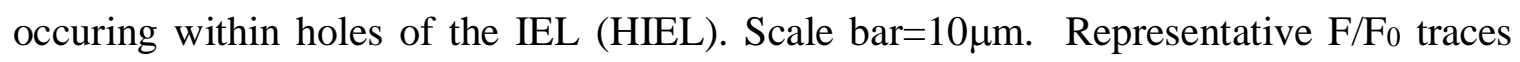
show calcium release events occurring before and after CCh or PE. E, Quantification of the absolute number of calcium events occurring at HIEL before and after CCh stimulation. F, Percent increase in calcum events with CCh compared to baseline. G, Quantification of absolute number of calcium events before and after PE stimulation. $\mathbf{H}$, Percent increase in calcium events with PE compared to baseline. (EC Calr fl/fl n=5 arteries from 5 mice, EC Calr $\Delta / \Delta \mathrm{n}=5$ arteries from 5 mice). ${ }^{*} \mathrm{p}<0.05, * * \mathrm{p}<0.01$ 


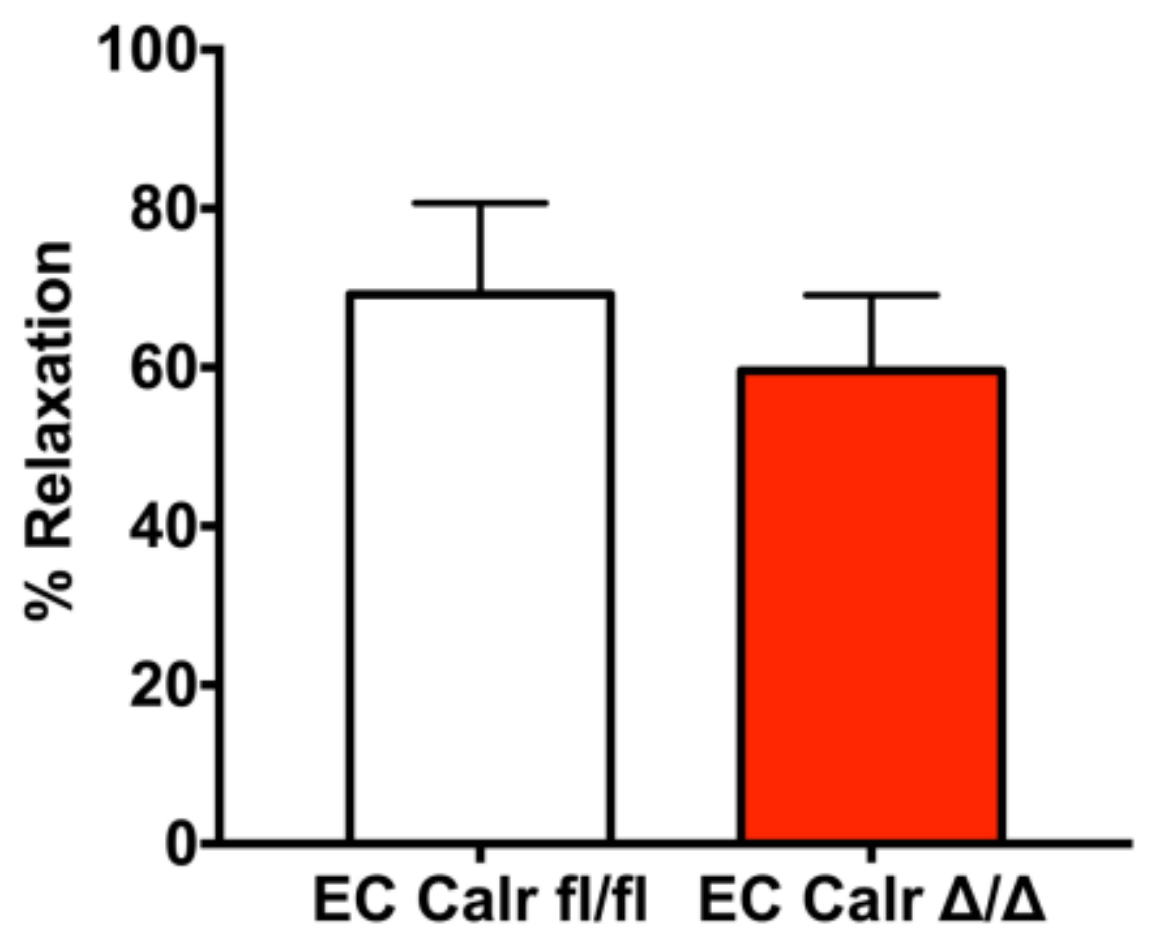

Figure 21. Dilation to $10 \mu \mathrm{M} C \mathrm{Ch}$ in EC Calr fl/fl and EC Calr $\Delta / \Delta$. Third order mesenteric arteries were allowed to develop tone and then a maximal dose of CCh was added to measure dilation. Only arteries that had tone and $1 \mu \mathrm{M}$ NS309 response were included. 
FIGURE 22. EC CALR ARTERIES HAVE DIFFERENTIAL VASOACTIVE RESPONSES TO ADRENERGIC VERSUS MUSCARINIC AGONISTS THAT RESULTS IN HIGHER MEAN ARTERIAL PRESSURE

A

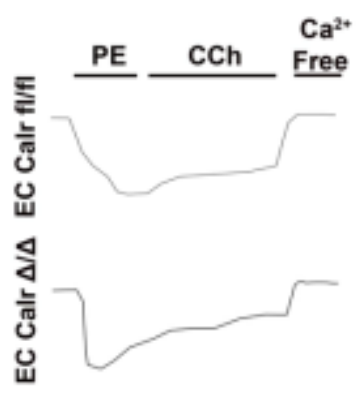

C

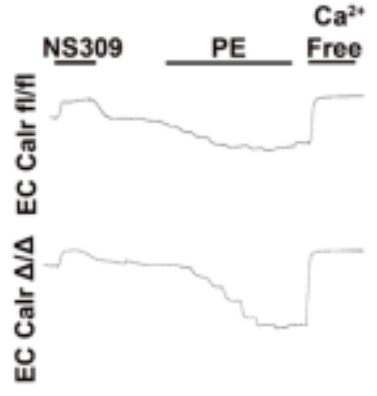

E
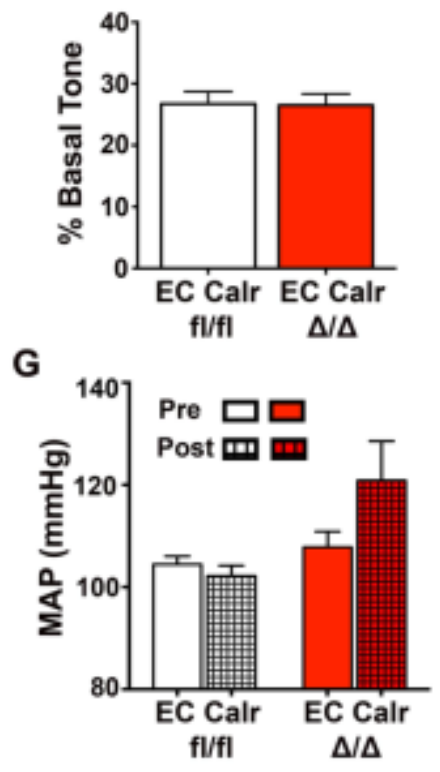

B

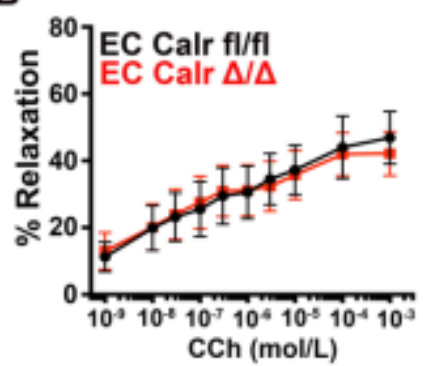

D

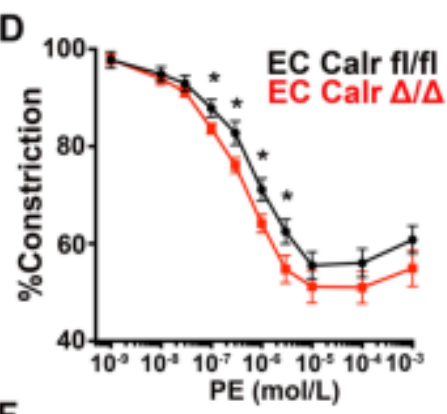

$\mathbf{F}$

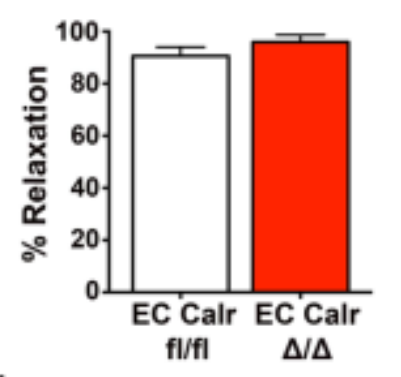

H

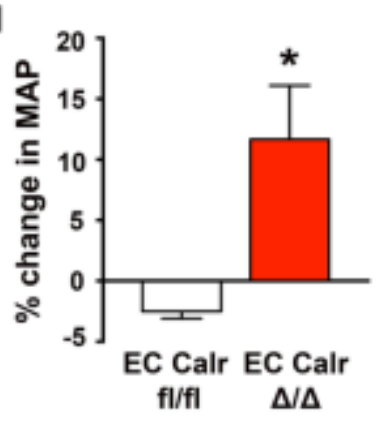


Figure 22. EC Calr $\Delta / \Delta$ arterioles have differential vasoreactive responses to adrenergic versus muscarinic agonists that results in a higher mean arterial pressure. Third order mesenteric arterioles were dissected clean of fat, cannulated and pressurized to $80 \mathrm{mmHg}$. Arterioles were maintained at $37^{\circ}$. A, Representative lumen diameter traces to carbachol $(\mathrm{CCh})$ dose response. B, Quantification of diameter response to $\mathrm{CCh}$ (EC Calr fl/fl $\mathrm{n}=3$; EC Calr $\Delta / \Delta \mathrm{n}=3$ ). $\mathbf{C}$, Representative lumen diameter traces to phenylephrine (PE). D, Quantification of diameter response to PE (EC Calr fl/fl $n=12$ arteries from 8 mice; EC Calr $\Delta / \Delta \mathrm{n}=11$ arteries from 8 mice; $\left.{ }^{*} \mathrm{p}<0.06\right)$. Smooth muscle cell (SMC) function and viability was assessed via $\mathbf{E}$, initial tone at $80 \mathrm{mmHg}$ (EC Calr fl/fl $\mathrm{n}=12$ arteries from 8 mice; EC Calr $\Delta / \Delta \mathrm{n}=11$ arteries from 8 mice). F, NS309 $(1 \mu \mathrm{M})$ dilation indicates intact signaling at the myoendothelial junction (MEJ) along with endothelial viability (EC Calr fl/fl $\mathrm{n}=12$ arteries from 8 mice; EC Calr $\Delta / \Delta \mathrm{n}=11$ arteries from 8 mice.) G, Radiotelemetry catheters were implanted and daily averages for mean arterial pressure (MAP) were recorded over the course of 50 days. Each mouse was compared back to its initial baseline MAP. The average MAP 5 days prior to injections (left, solid bars) versus after injections (right, patterned bars). H, Percent change from baseline to the end of the study (EC Calr fl/fl $\mathrm{n}=3$; EC Calr $\Delta / \Delta \mathrm{n}=3$ ). ${ }^{*} \mathrm{p}<0.05$ 
FIGURE 23. ACUTE MEASUREMENT OF NIGHTTIME BLOOD PRESSURE.

A
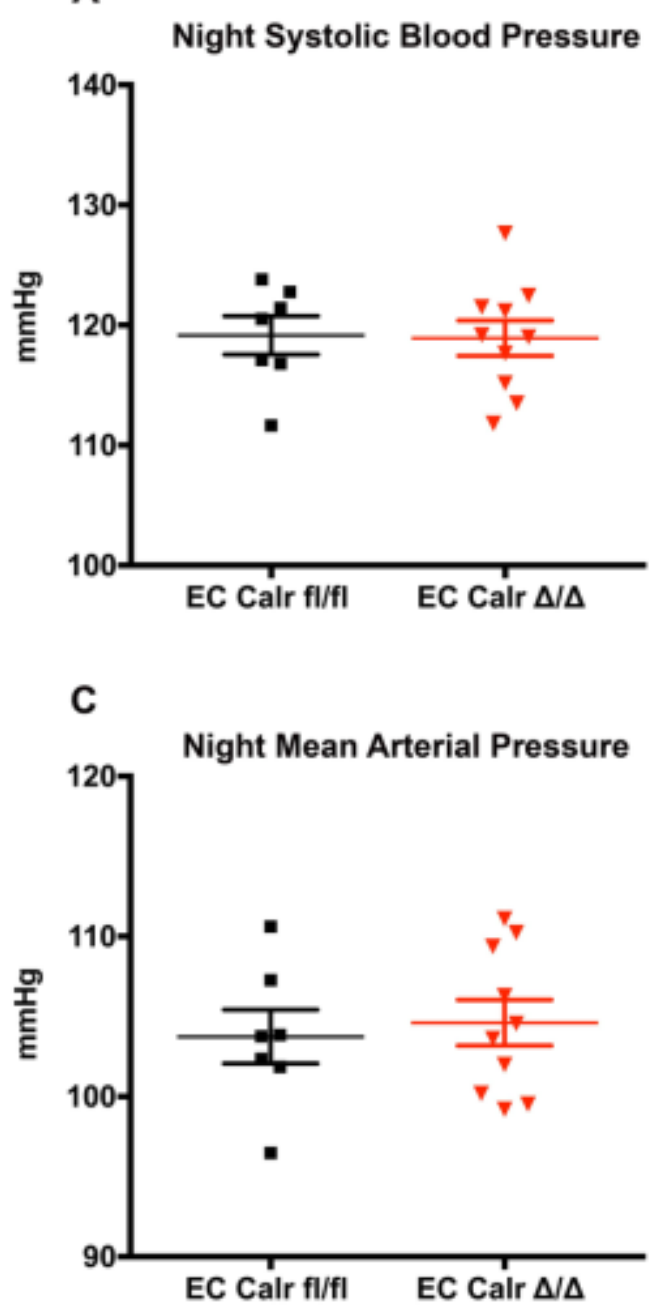

B
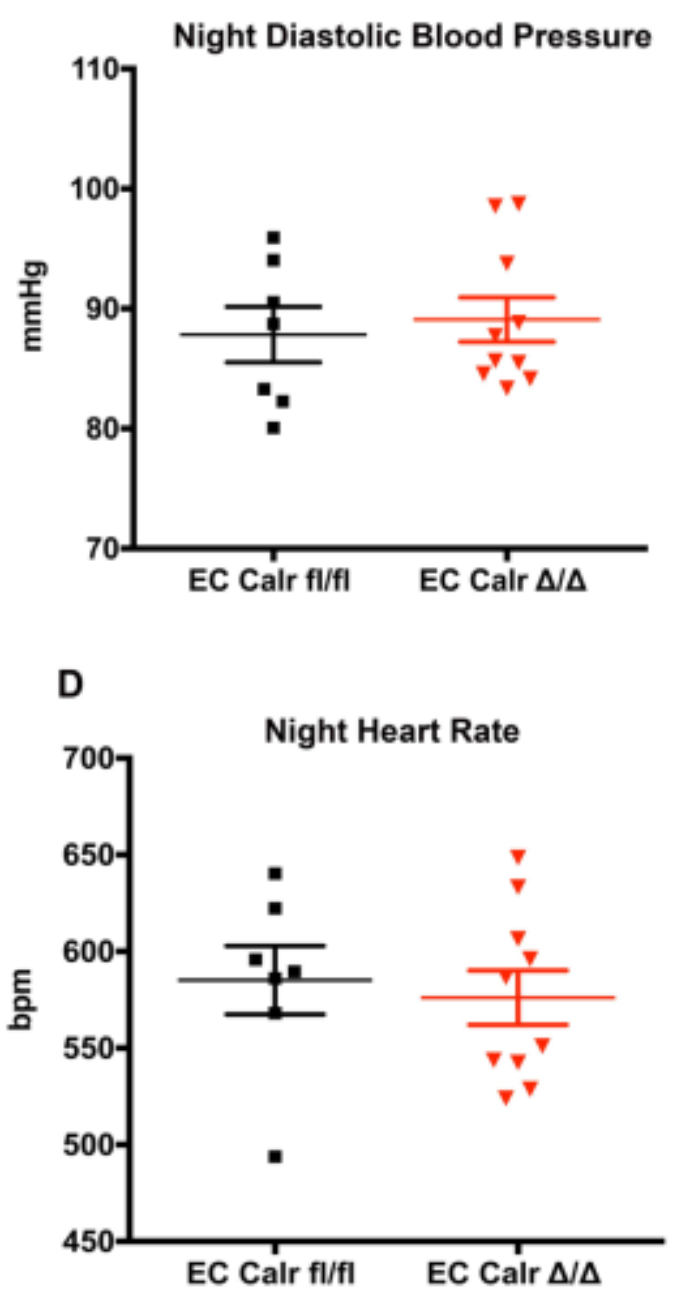

Figure 23. Acute measurement of nighttime blood pressure. Pressures and heart rate were measured and averaged during the dark cycle when mice were most active for 4 days. There was no difference in $\mathbf{A}$, systolic blood pressure $\mathbf{B}$, diastolic blood pressure $\mathbf{C}$, mean arterial pressure or $\mathbf{D}$, heart rate. (EC Calr fl/fl $n=7$, EC Calr $\Delta / \Delta \mathrm{n}=10$ ) 
FIGURE 24. ACUTE BLOOD PRESSURE RESPONSE TO A PHENYLEPHRINE (PE) INJECTION

A Systolic Blood Pressure

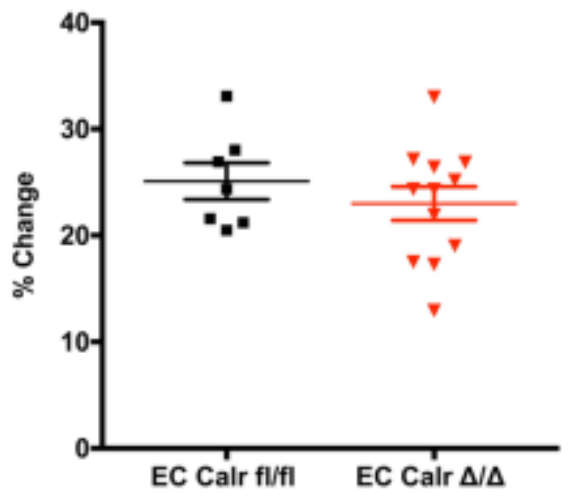

C Mean Arterial Pressure

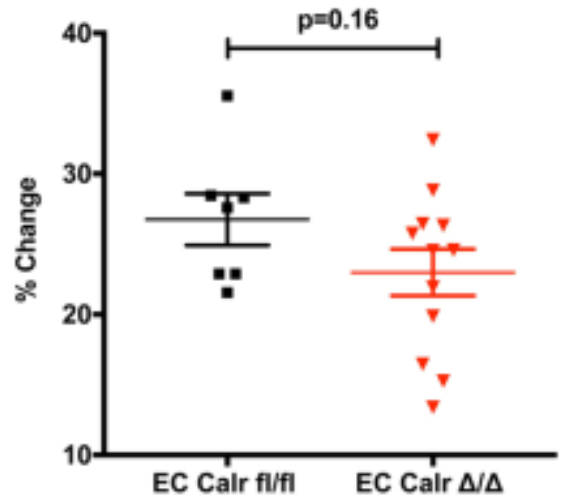

B Diastolic Blood Pressure

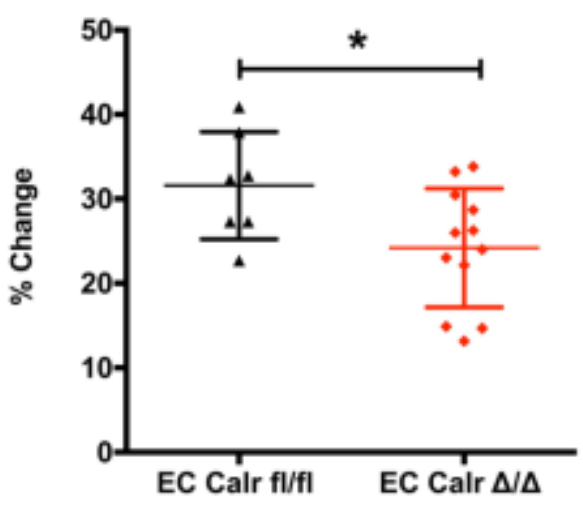

Heart Rate

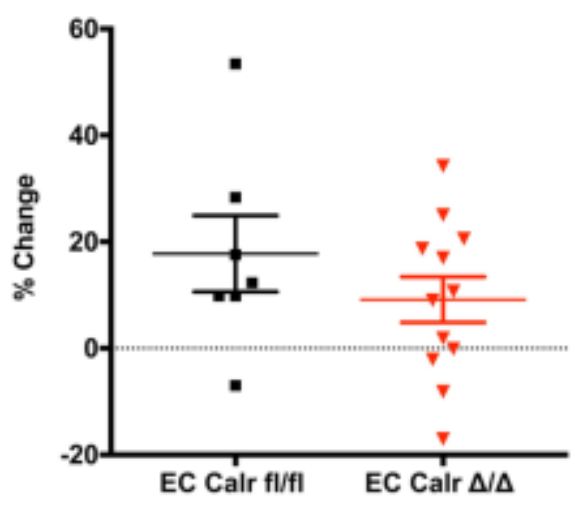

Figure 24. Acute blood pressure response to a phenylephrine (PE) injection. Pressures and heart rate were measured to obtain a two hour baseline prior to injection. Mice were injected with $0.1 \mathrm{mg} / \mathrm{kg}$ PE and the average change in the first 15 minutes after injection is presented. A, Systolic blood pressure was not different. B, Diastolic blood pressure response to $\mathrm{PE}$ was significantly decreased in $\mathrm{EC}$ Calr $\Delta / \Delta$ mice. $\mathbf{C}$, Mean arterial pressure was not significantly different between groups. D, Heart rate was not different. (EC Calr fl/fl $n=7$, EC Calr $\Delta / \Delta n=12$ ) 
FIGURE 25. TIME COURSE OF BLOOD PRESSURE RESPONSE TO ANGIOTENSIN II (ANG II)

A

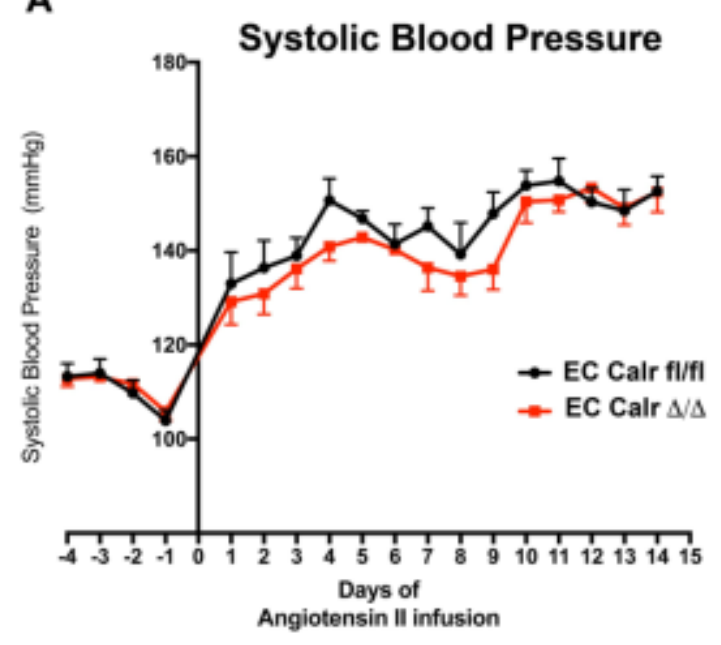

C

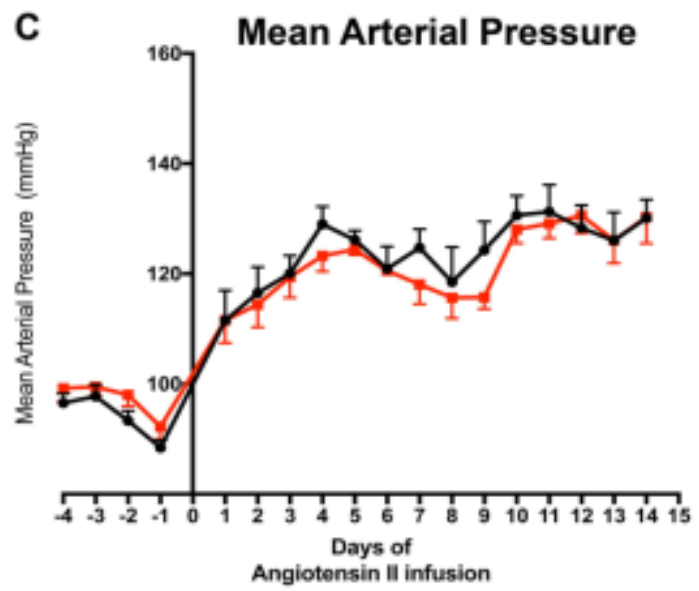

B
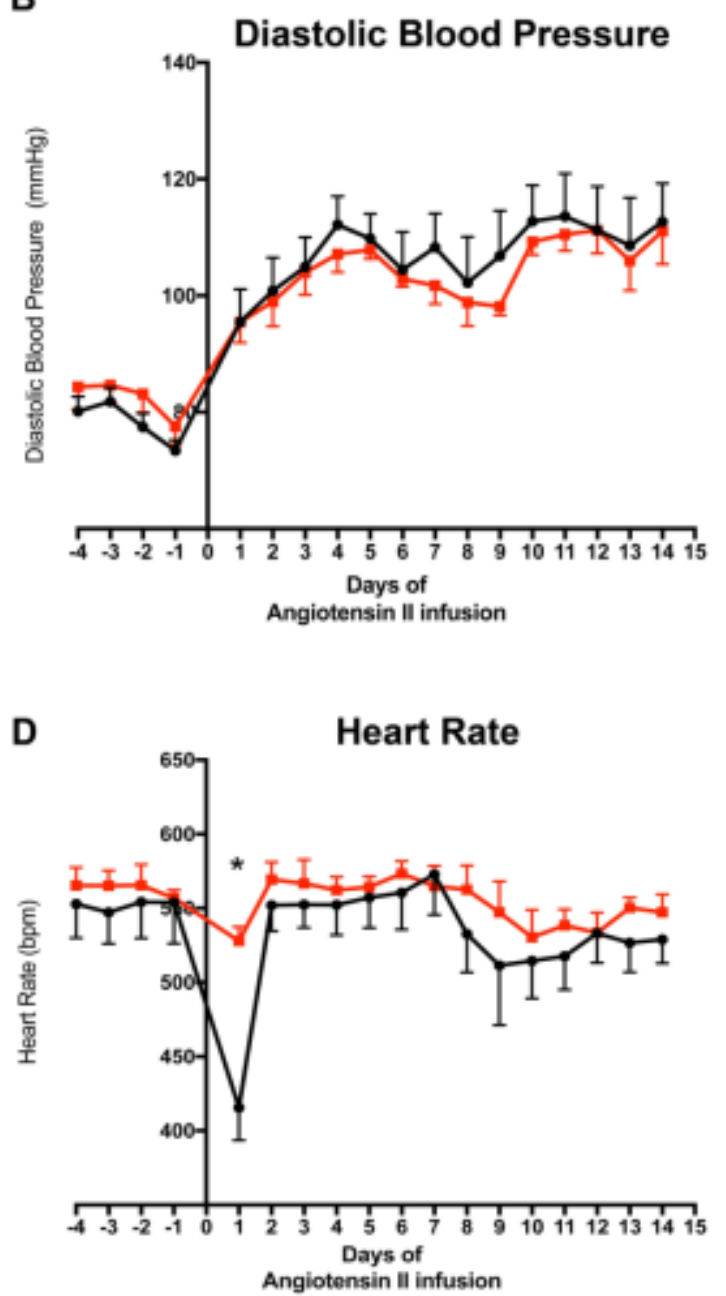
Figure 25. Time course of blood pressure response to angiotensin II (AngII). Pressures and heart rate were measured before and during AngII administration. A, Systolic blood pressure $\mathbf{B}$, diastolic blood pressure and $\mathbf{C}$, mean arterial pressure baseline values (days -4 to 0) and AngII response (Days 1-15) were not significantly different. D, Heart rate decreased with Ang II in EC Calr fl/fl mice on day 1, but that decrease was significantly blunted in EC Calr $\Delta / \Delta$. (EC Calr fl/fl $n=4$, EC Calr $\Delta / \Delta \mathrm{n}=4)$ 
A

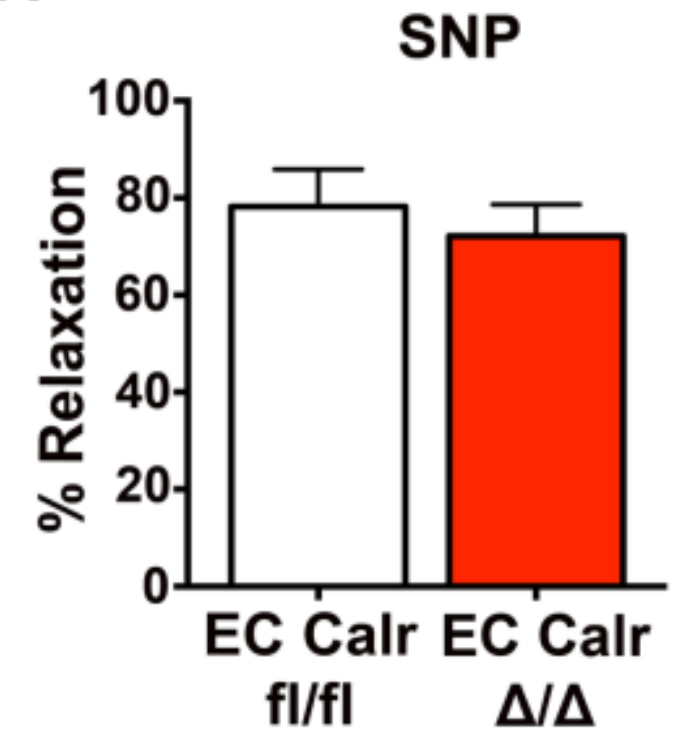

B

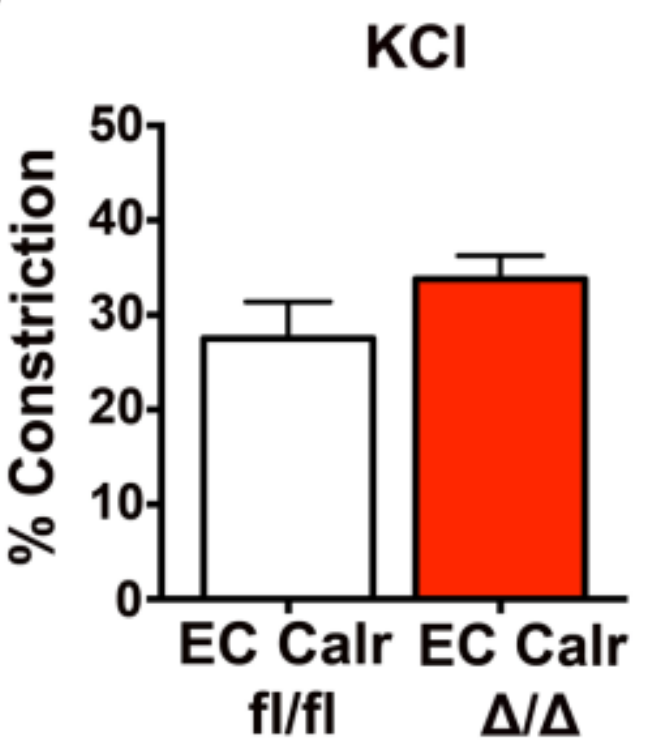

Figure 26. EC Calr $\Delta / \Delta$ does not change endothelial-independent dilation or smooth muscle constriction to $\mathbf{K C l}$. A, Endothelial independent dilation was assessed via $1 \mathrm{mM}$ sodium nitroprusside (SNP; EC Calr fl/fl $\mathrm{n}=9$ arteries from 7 mice; EC Calr $\Delta / \Delta \mathrm{n}=6$ arteries from 5 mice) $\mathbf{B}$, SMC constriction was assessed using $40 \mathrm{mM} \mathrm{KCl} \mathrm{(EC} \mathrm{Calr} \mathrm{fl/fl}$ $\mathrm{n}=6$ arteries from 5 mice; EC Calr $\Delta / \Delta \mathrm{n}=8$ arteries from 8 mice). 
FIGURE 27. TIME COURSE OF AVERAGE DAILY BLOOD PRESSURE AND HEART RATE

A

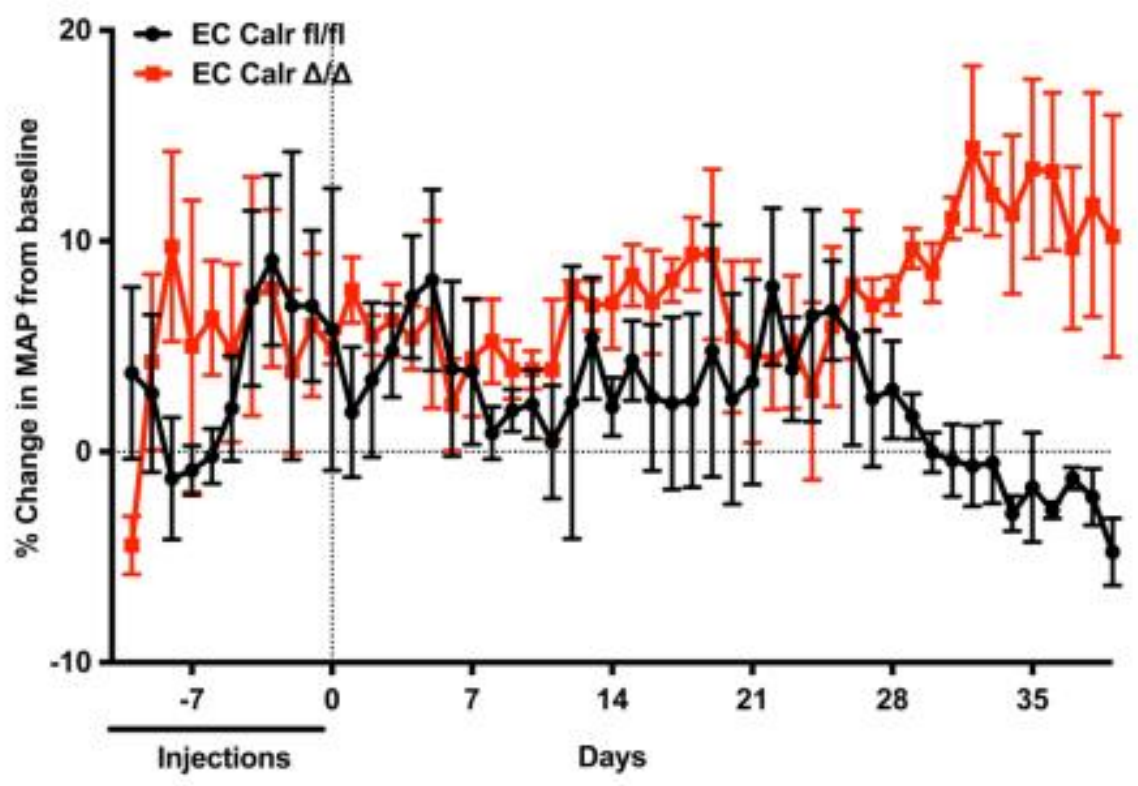

B

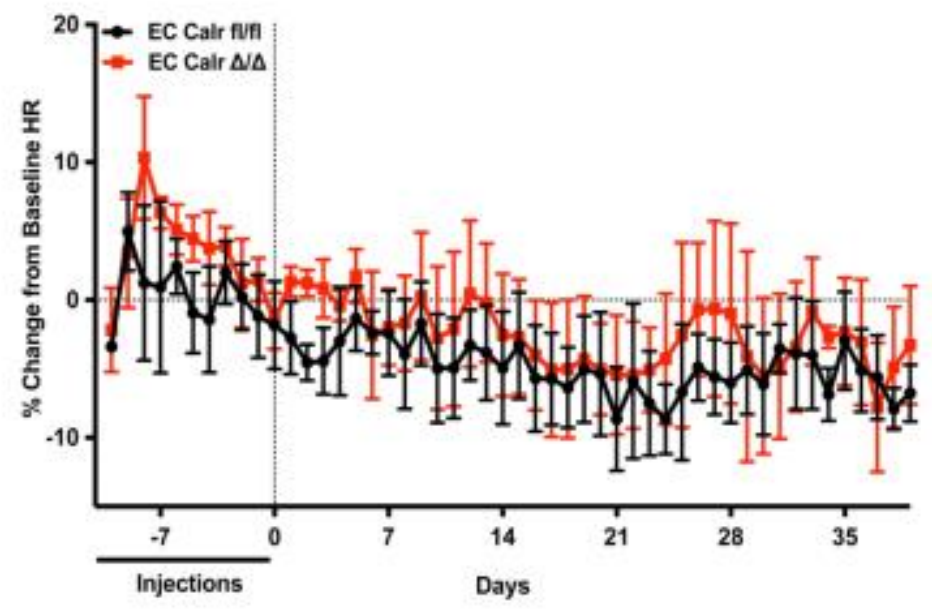

C

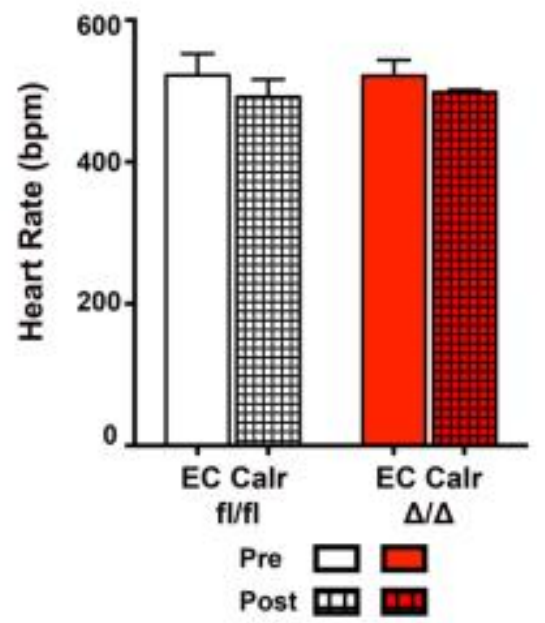


Figure 27. Time course of average daily blood pressure and heart rate. The average daily change in $\mathbf{A}$, mean arterial pressure (MAP) and $\mathbf{B}$, heart rate compared to the preinjection baseline measured via radiotelemetry catheters. Days -10 to -1 indicate injection with tamoxifen or vehicle control. Days 0-39 indicate post-EC Calr knockout (Days 0-39). C, Baseline absolute heart rate was not different. 
FIGURE 28. CALR KNOCKDOWN IN EC DOES NOT AFFECT THE LEVEL OF ER CALCIUM

A

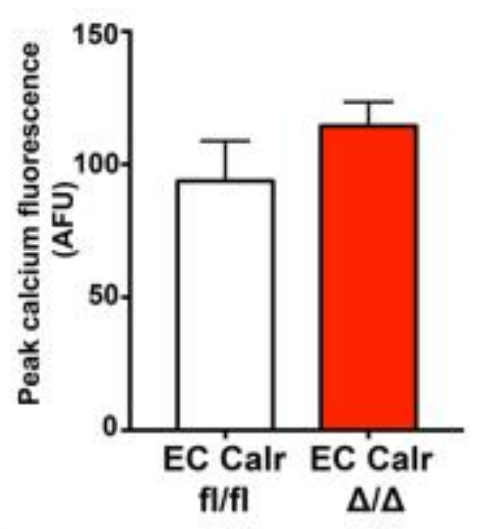

C

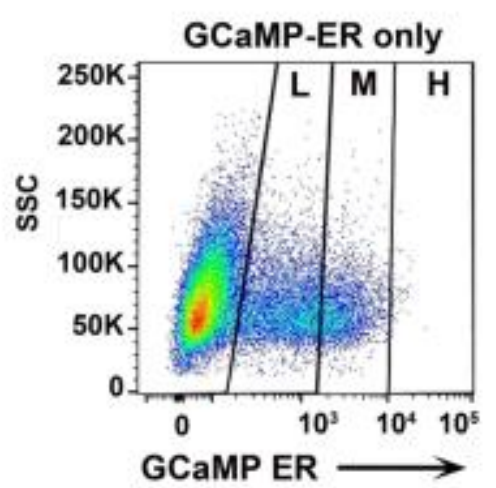

B



D

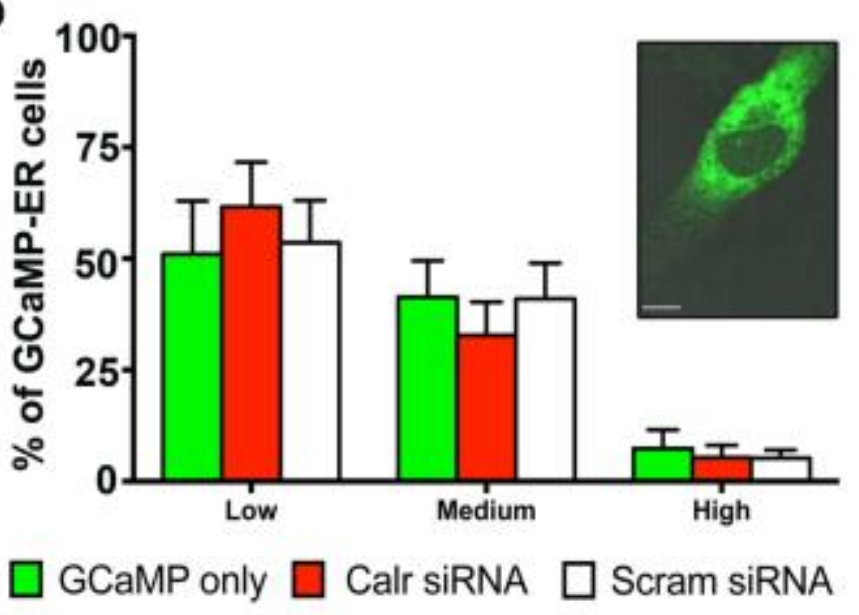


Figure 28. Calr knockdown in EC does not affect the level of ER calcium. A, Third order mesenteric arterioles from EC Calr fl/fl and EC Calr $\Delta / \Delta$ mice were loaded with Fluo4AM. Arterioles were incubated in $0 \mathrm{mM}$ extracellular calcium, along with cyclopiazonic acid $(20 \mu \mathrm{M})$ to inhibit SERCA refilling of ER calcium stores. This provides an indirect measurement of ER calcium stores (EC Calr fl/fl n=3; EC Calr $\Delta / \Delta \mathrm{n}=3$ ). B, Representative western blot of primary human aortic EC, which were transfected with GCaMP-ER plasmid to specifically detect ER calcium fluorescence. Then EC were transfected with either calreticulin siRNA or scrambled siRNA. Knockdown was assessed via western blot for calreticulin. Values were normalized to total protein for each lane and compared to scrambled siRNA. Control EC were untransfected. MW=molecular weight ladder. $\mathbf{C}$, Representative flow cytometry plot for EC transfected with GCaMP-ER to visualize ER calcium, divided into low (L), medium $(\mathrm{M})$ and high $(\mathrm{H})$ fluorescent signal. SSC=side scatter. D, Quantification of GCaMP-ER signal for EC co-transfected with Calr or scrambled siRNA. Inset: Representative confocal image of an EC with GCaMP-ER signal (green, visualized with GFP laser) indicating calcium within the ER. (GCaMP-ER only n=3, Calr siRNA n=3, Scram siRNA n=3) Scale bar=10 $\mu \mathrm{m}$ 
FIGURE 29. GCAMP-ER FLOW CYTOMETRY GATING STRATEGY AND REPRESENTATIVE RESULTS

A

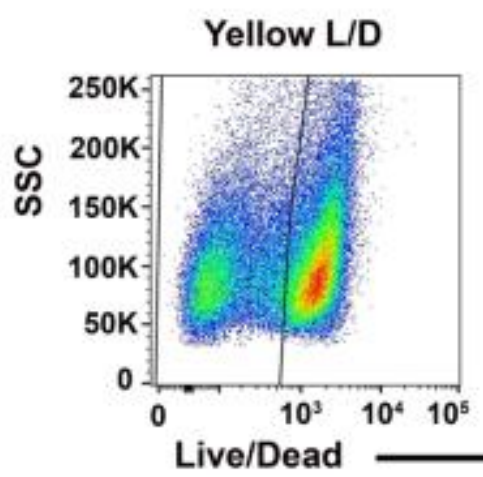

B

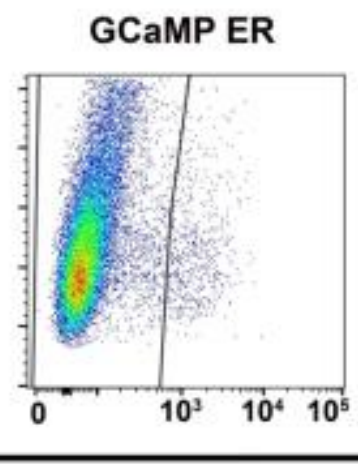

C GCaMP + Yellow
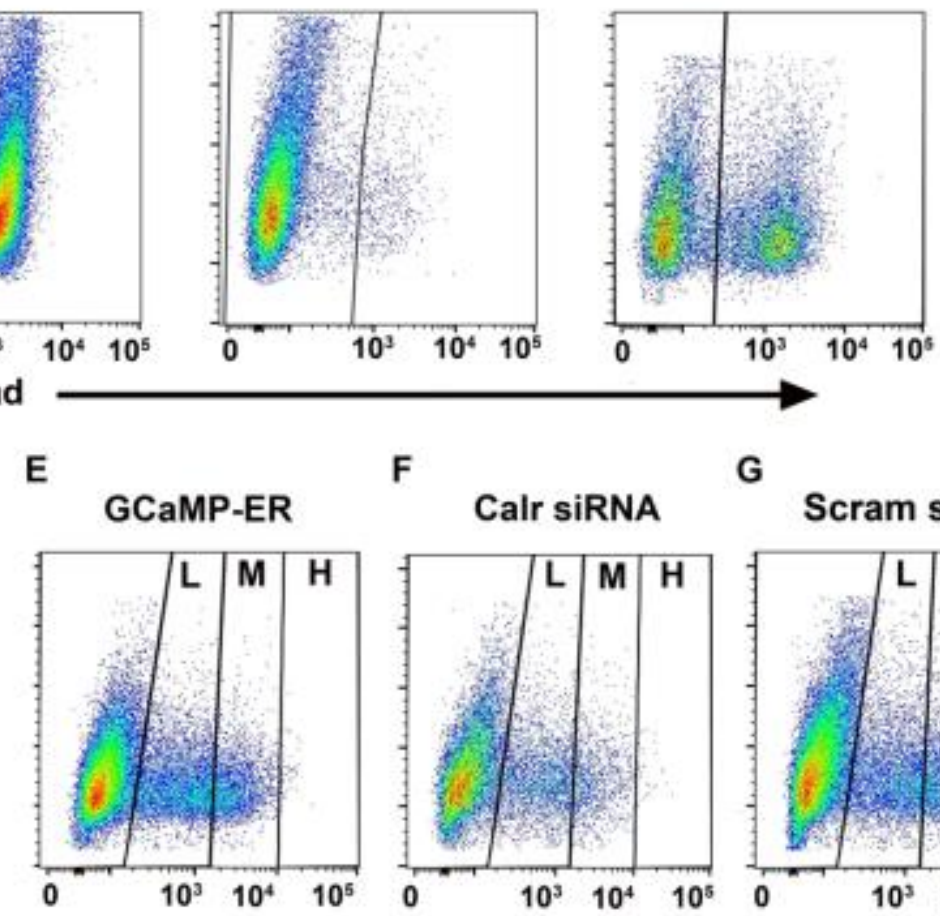

F

G Calr siRNA

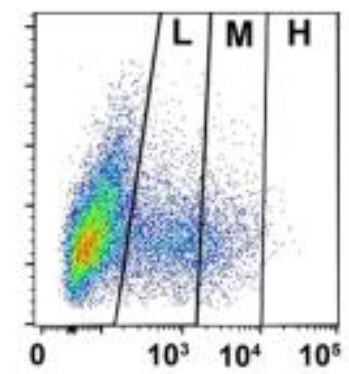

Scram siRNA

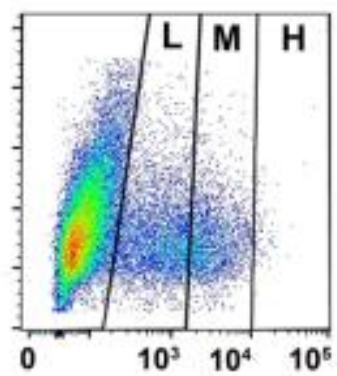
GCaMP ER Fluorescence 
Figure 29. GCaMP-ER flow cytometry gating strategy and representative results. Because GCaMP-ER fluorescence may change with apoptotic or dying cells, only live EC were analyzed. A, EC stained with yellow live/dead fluorescent dye (L/D). The dye is not taken up by cells with intact membranes, so increased fluorescence indicates non-viable cells. B, EC transfected with only GCaMP-ER do not have positive signal for live/dead dye. C, When EC transfected with GCaMP-ER are incubated with live/dead dye, a population of dead cells is seen in the right portion of the graph. D, Untransfected EC stained with yellow live/dead dye do not have signal for GCaMP-ER. E, EC transfected with GCaMP-ER and F, Calr siRNA or $\mathbf{G}$, scrambled siRNA do not exhibit significant differences in low (L), medium (M) or high (H) GCaMP-ER fluorescence. Cell populations in the far left box do not exhibit GCaMP-ER signal. SSC= side scatter. 
FIGURE 30. GCAMP-ER SIGNAL DECREASES AFTER ADDITION OF ATP

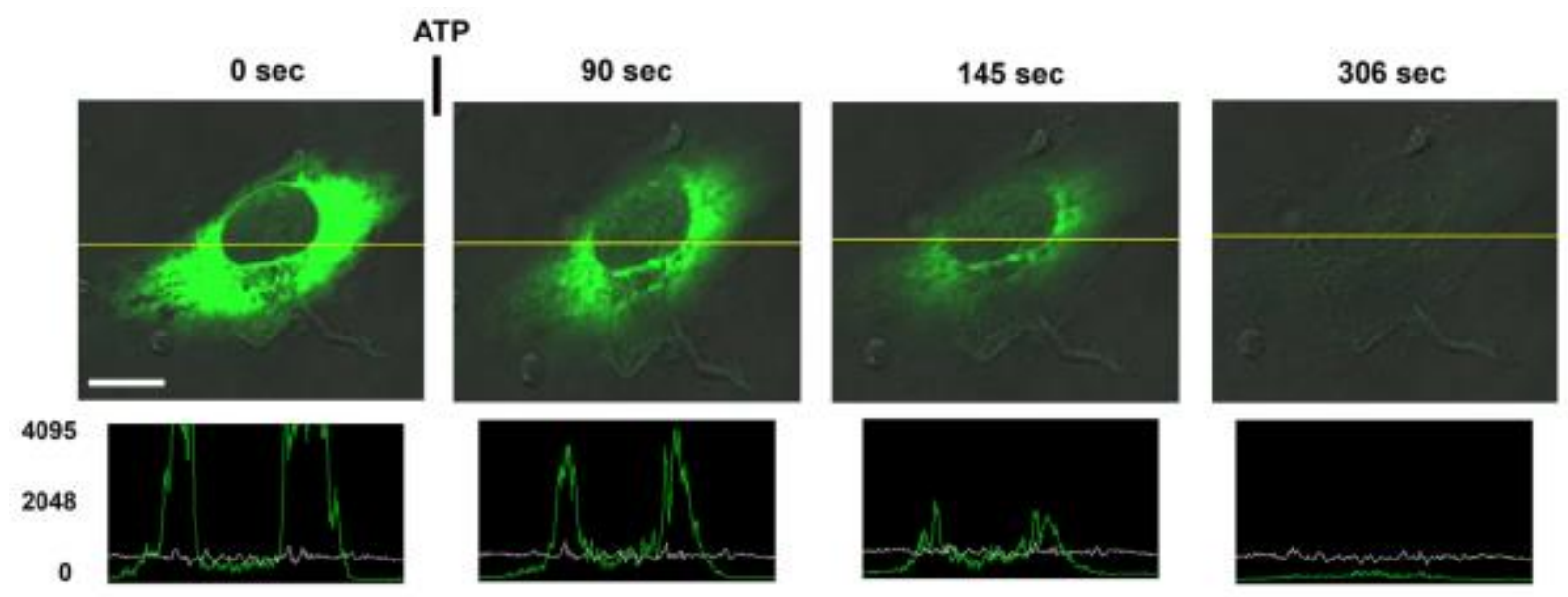

Figure 30. GCaMP-ER signal decreases after addition of $35 \mu \mathrm{M}$ ATP. Time-lapse images of an EC transfected with GCaMP-ER (green, visualized via GFP 488 laser) imaged on an upright confocal microscope over the course of 5 minutes. Line scans are indicated with yellow line and the fluorescent intensity is plotted below the image at each timepoint (Green is GCaMP-ER signal, grey is brightfield channel). Scale bar $=10 \mu \mathrm{m}$. 
FIGURE 31. TIME COURSE OF CALR SIRNA KNOCKOUT EFFICIENCY

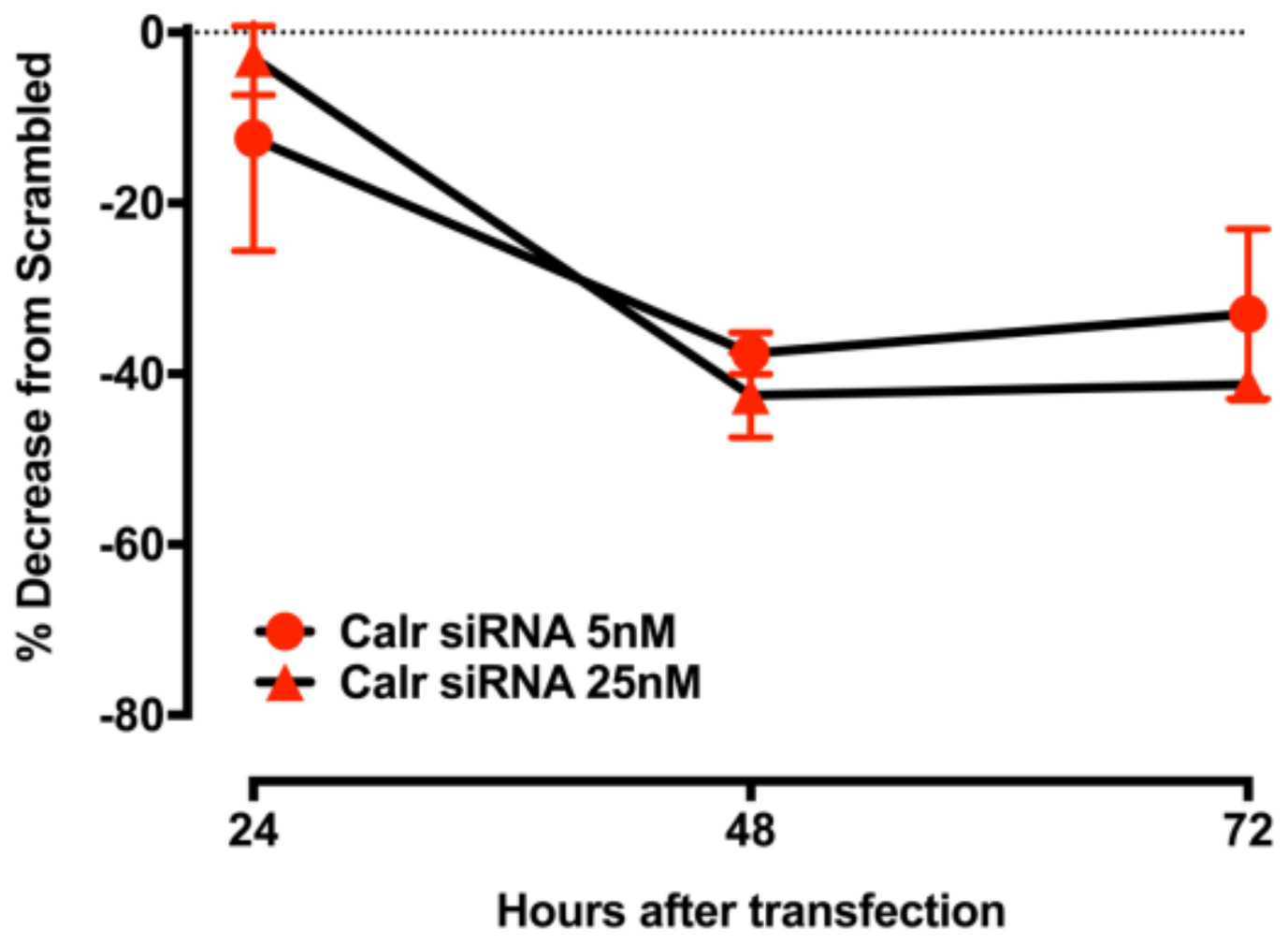

Figure 31. Time course of Calr siRNA knockout efficiency. Primary human EC were transfected with either Calr siRNA or scrambled siRNA at 5nM and 25nM concentrations and harvested after 24,48 , or 72 hours. There was no difference between low and high concentrations of Calr siRNA at any of the timepoints. The maximum decrease seen was at 48 hours ( $40 \%$ versus scrambled), which persisted at 72 hours. (Calr $5 \mathrm{nM} 24 / 48 / 72 \mathrm{hr}$ $\mathrm{n}=2$, Calr 25nM 24/48/72hr n=2, Scram 5nM 24/48/72hr n=2, Scram 25nM 24/48/72hr $n=2)$ 


\section{FIGURE 32. IN VITRO CALR CO-IMMUNOPRECIPITATION SHOWS} DIFFERENTIAL INTERACTION WITH PROTEINS IN EC MONOLAYER VERSUS THE MEJ

A

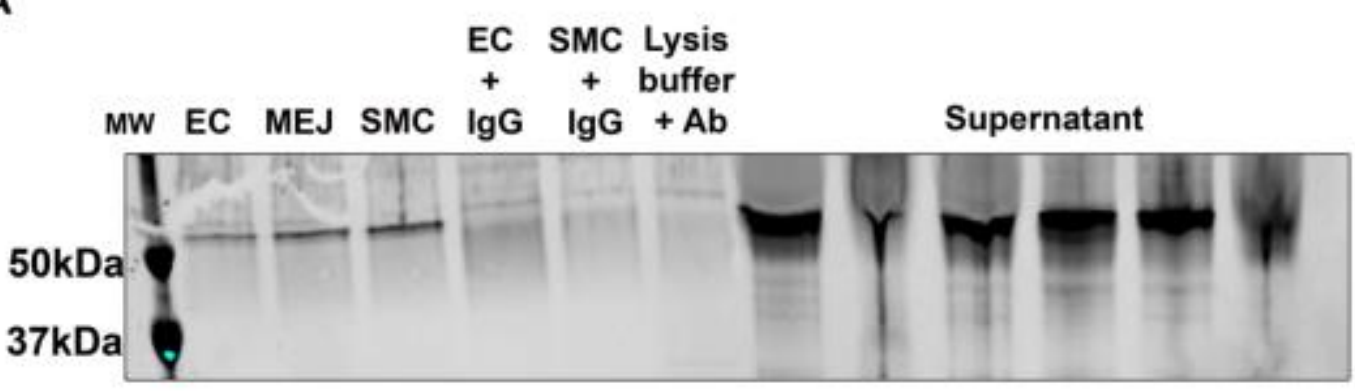

\begin{tabular}{|l|l|l|l|l|l|}
\hline \multicolumn{1}{|c|}{ Protein } & $\begin{array}{c}\text { Accession } \\
\text { Number }\end{array}$ & $\begin{array}{c}\text { Molecular } \\
\text { Weight } \\
\text { (kDa) }\end{array}$ & EC & MEJ & SMC \\
\hline $\begin{array}{l}\text { 78kDa Glucose } \\
\text { regulated protein }\end{array}$ & P11021 & 72 & 7 & 1 & 1 \\
\hline $\begin{array}{l}\text { Trio and factin } \\
\text { binding protein } \\
\text { OS }\end{array}$ & Q9H2D6 & 261 & 0 & 0 & 2 \\
\hline $\begin{array}{l}\text { Inositol- } \\
\text { triphosphate } \\
\text { kinase A }\end{array}$ & P23677 & 51 & 0 & 1 & 0 \\
\hline $\begin{array}{l}\text { Thrombospondin- } \\
1\end{array}$ & P07996 & 129 & 1 & 2 & 0 \\
\hline
\end{tabular}


Figure 32. In vitro Calr co-immunoprecipitation shows differential interaction with proteins in EC monolayer versus the MEJ. Primary human endothelial and smooth muscle cells were grown on opposite sides of a filter with $0.4 \mu \mathrm{m}$ pores in which MEJ can form. These three fractions were isolated, lysed and co-immunoprecipitated with Calreticulin antibody in order to find binding partners of calreticulin. A, Representative immunoblot of calreticulin co-immunoprecipitation samples. B, Table indicating two proteins that interact with Calreticulin in the vascular cell co-culture and their spectral counts, a semiquantitative measurement of protein abundance. 
FIGURE 33. PE DOSE RESPONSE WITH BREFELDIN A (BFA)

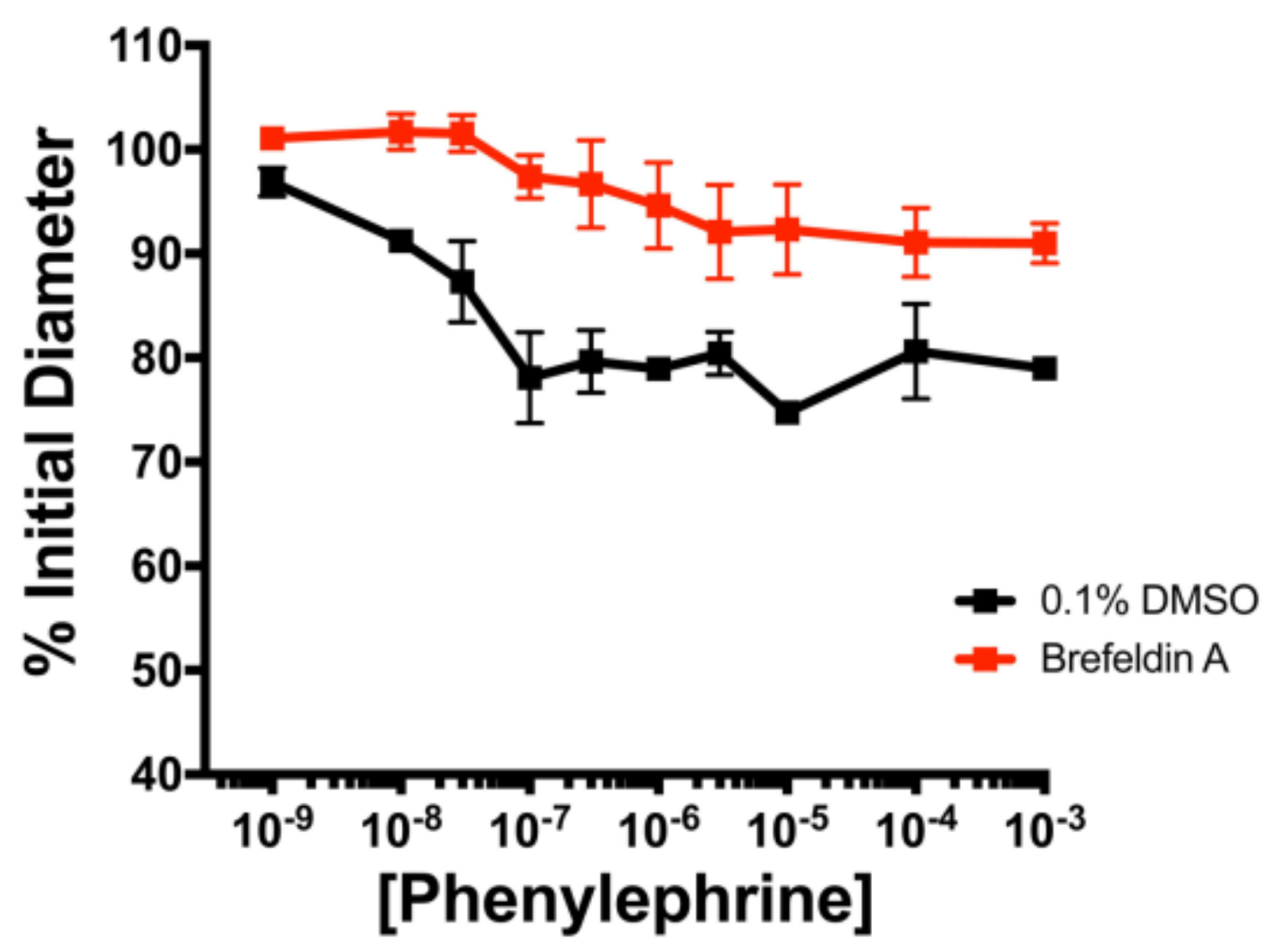

Figure 33. PE dose response with Brefeldin A (BFA). Third order mesenteric arteries from $\mathrm{C} 57 \mathrm{Bl} / 6$ mice were incubated with BFA or $0.1 \%$ DMSO as a control. Using pressure myography, arteries underwent PE dose response in the presence of BFA. Only arteries that developed tone and dilated to NS309 were used. (DMSO $n=2$ arteries, BFA $n=4$ arteries) 
FIGURE 34. CALR AT MEJ MAY BE LOCALIZED OUTSIDE THE ER
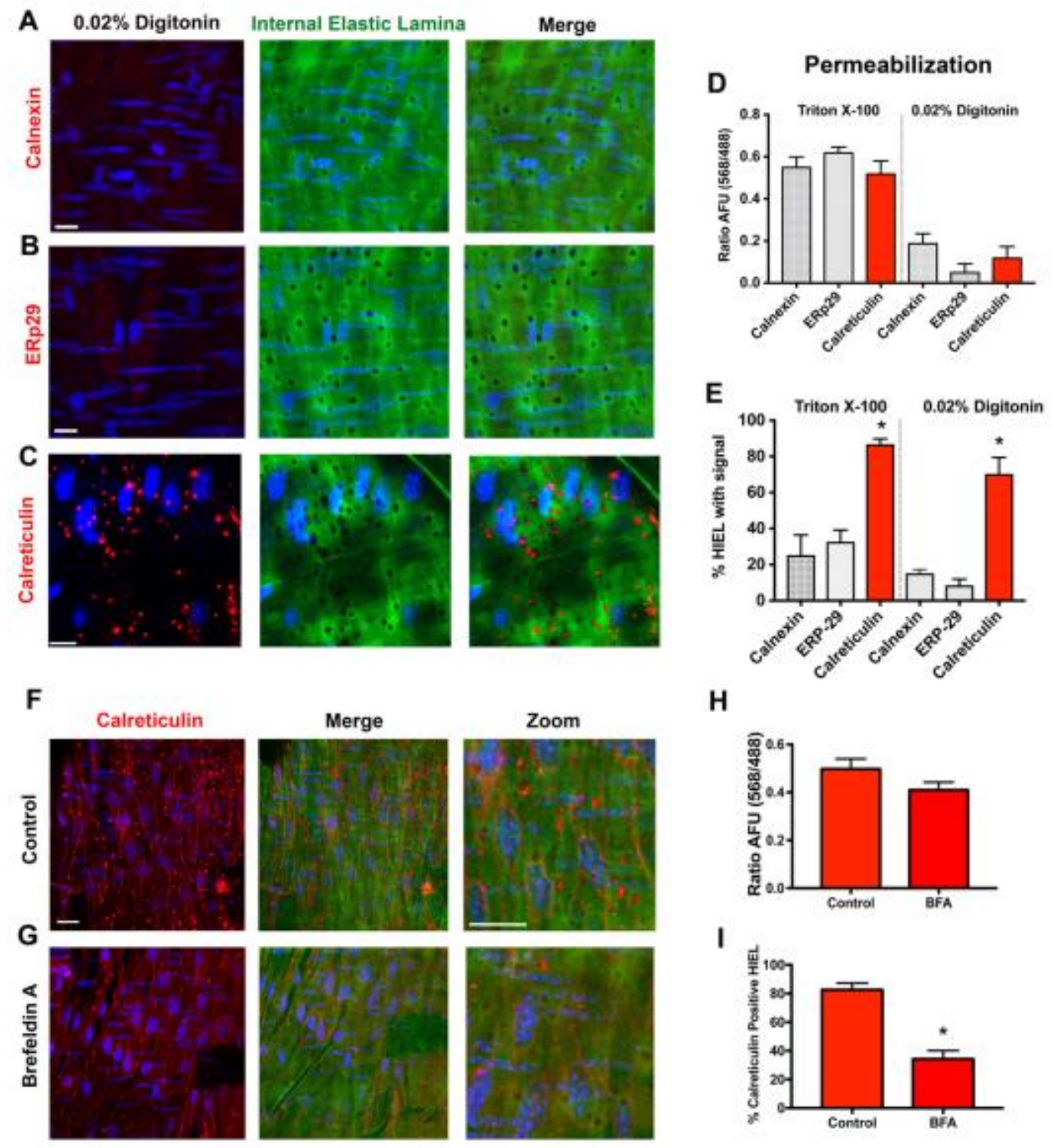

H
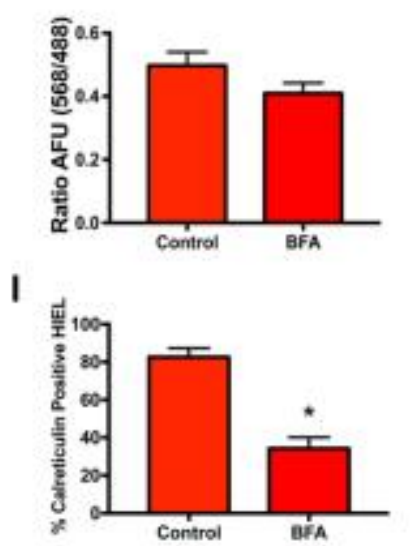
Figure 34. Calr at MEJ may be localized outside the ER. Representative en face immunofluorescence for A, calnexin B, ERp29 and C, Calr (red) with $0.02 \%$ digitonin permeabilization. This selectively permeabilizes plasma membrane and not the intracellular ER membrane. D, Quantification of EC monolayer fluorescence (568 nm) normalized to IEL autofluorescence $(488 \mathrm{~nm})$ in arbitrary fluorescent units (AFU). E, Percentage of holes in the IEL (HIEL) with positive staining. (Calreticulin/ERp29/calnexin n=3) Representative arterioles were incubated $\mathbf{F}$, without or $\mathbf{G}$, with Brefeldin A (BFA; $1 \mu \mathrm{g} / \mathrm{mL}$ ), fixed with $4 \%$ paraformaldehyde, and stained for Calr (red). Scale bar $=10 \mu \mathrm{m}$. H, Quantification of EC monolayer fluorescence (568) normalized to IEL autofluorescence (488) in AFU. I, Percentage of HIEL with calreticulin signal. (Control/BFA n=4) *p<0.05. 
FIGURE 35. CALCIUM SIGNALS IN AGED EC CALR $\Delta / \Delta$ ARE DIMINISHED WITH AGE

A

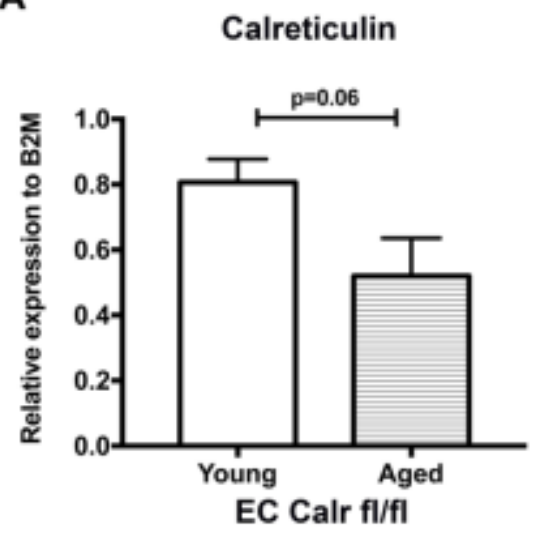

B

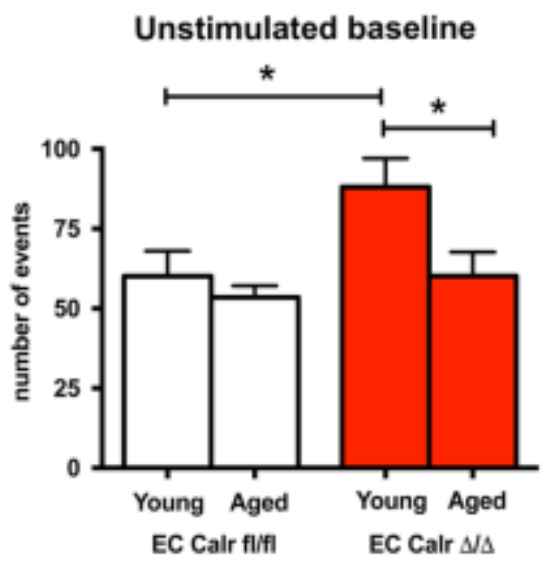

C

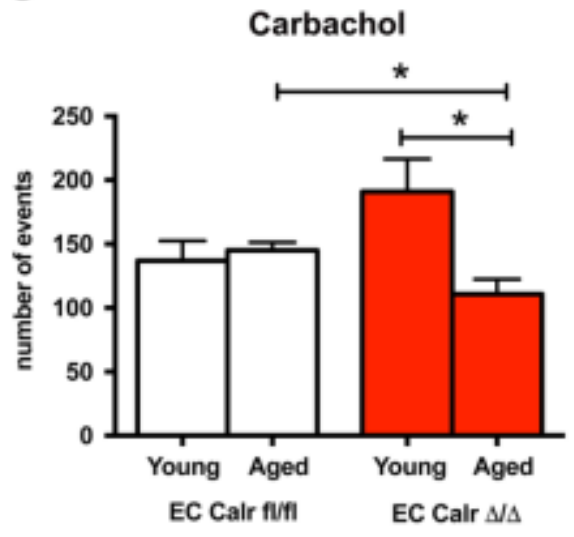

D

Phenylephrine

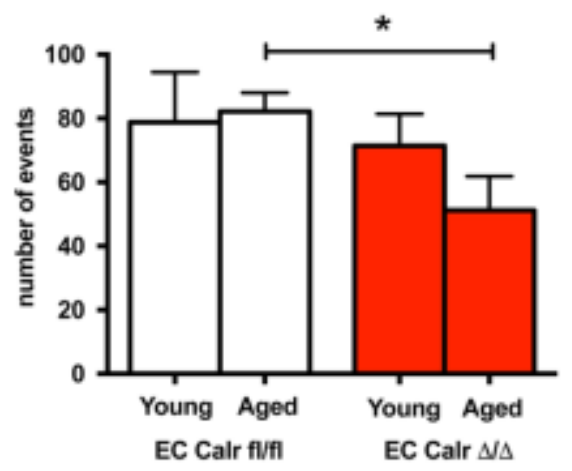


Figure 35. Calcium signals in EC Calr $\Delta / \Delta$ arteries are diminished with age. A, Calr mRNA expression was decreased in thoracic aortas of aged ( $>75$ week old) versus young ( $<20$ week old) EC Calr fl/fl mice. B, Third order mesenteric arteries were loaded with Fluo-4 in the en face preparation and calcium events were measured at baseline and in response to $\mathrm{CCh}$ and PE. Young EC Calr $\Delta / \Delta$ had increased events at baseline. This difference disappeared with age. $\mathbf{C}$, Aged EC Calr $\Delta / \Delta$ mice had significantly fewer calcium events in response to CCh. D, Aged EC Calr $\Delta / \Delta$ arteries had significantly fewer events in response to PE (EC Calr fl/fl $\mathrm{n}=5$ arteries, EC Calr $\Delta / \Delta \mathrm{n}=5$ ). 
FIGURE 36. AGED EC CALR $\Delta / \Delta$ ARTERIES HAVE SIGNIFICANTLY DIMINISHED CALCIUM RESPONSES TO CCH AND PE VERSUS AGED EC CALR FL/FL
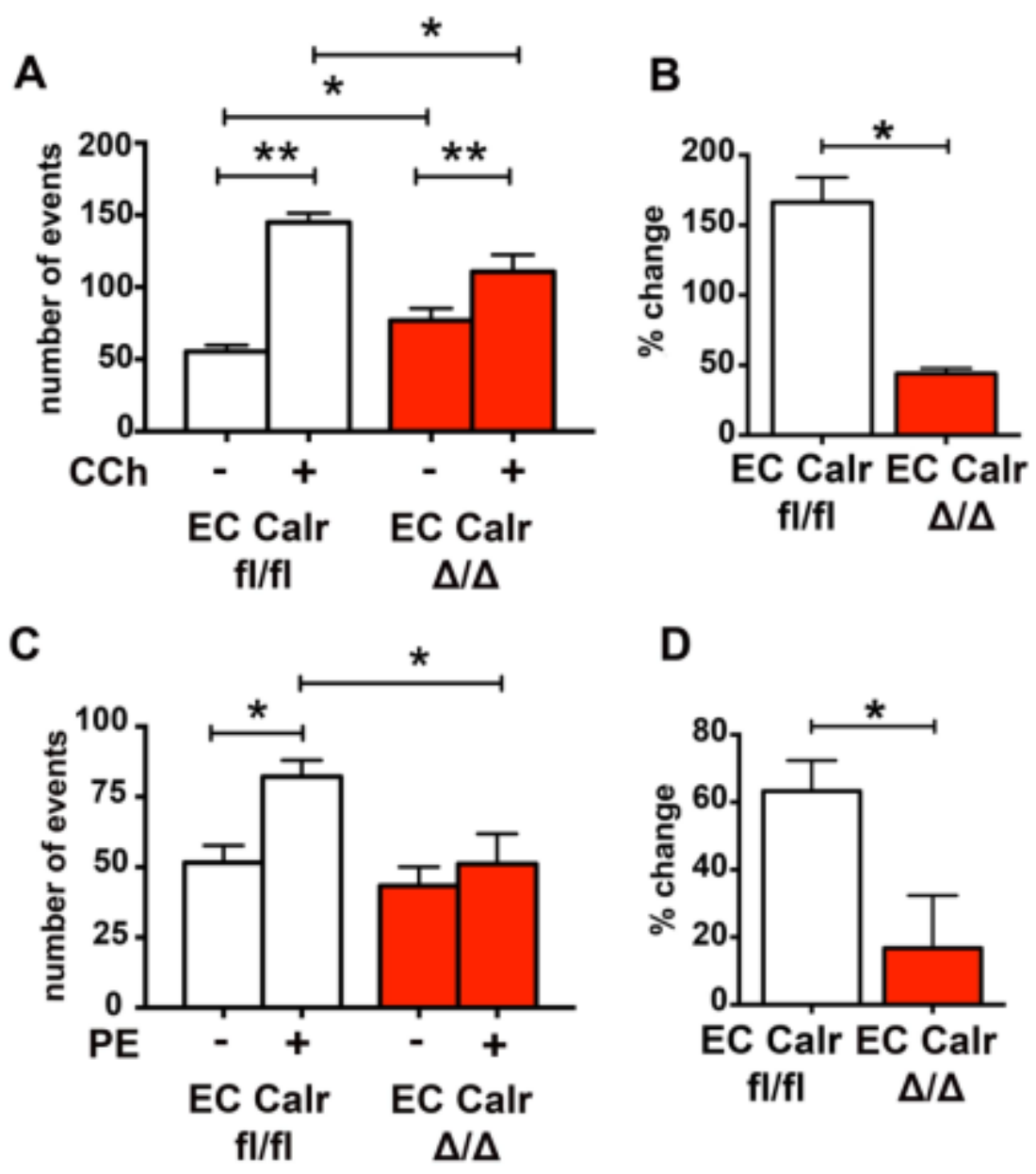
Figure 36. Aged EC Calr $\Delta / \Delta$ arteries have significantly diminished calcium responses to CCh and PE versus Aged EC Calr fl/fl. Third order mesenteric arteries from aged EC Calr fl/fl and $\Delta / \Delta$ (>75 weeks old) were loaded with Fluo-4 in the en face preparation and calcium events were measured at baseline and in response to CCh and PE. Only events that were within $5 \mu \mathrm{m}$ of IEL holes were quantified. A, CCh increased calcium events in both arteries, but EC Calr fl/fl had significantly fewer calcium events overall. B, The percent increase in calcium events to CCh was significantly lower in the EC Calr $\Delta / \Delta$. C, PE significantly increased calcium events in EC Calr fl/fl, but not EC Calr $\Delta / \Delta$. Furthermore, the total number of events in response to PE was significantly lower. D, PE increased calcium events $\sim 60 \%$ in EC Calr fl/fl arteries and this response was significantly lower in EC Calr $\Delta / \Delta($ EC Calr fl/fl $n=5$ arteries, EC Calr $\Delta / \Delta \mathrm{n}=5)$. 
FIGURE 37. INOSITOL TRIPHOSPHATE KINASE A (ITPKA) INTERACTS WITH CALR AT THE MEJ TO MEDIATE PE CONSTRICTION

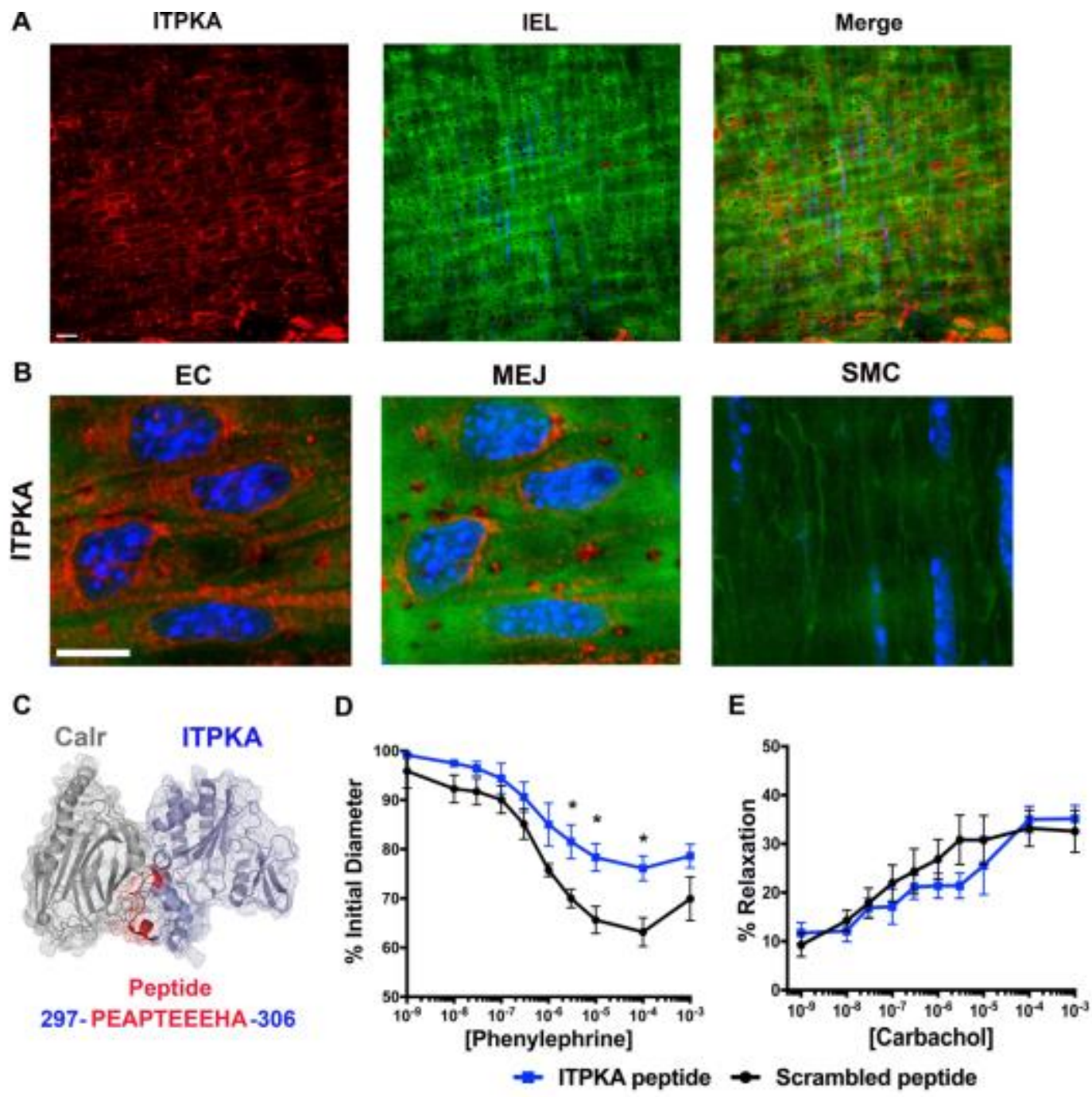


Figure 37. Inositol triphosphate kinase A (ITPKA) interacts with Calr at the MEJ to mediate PE constriction. A, B, En face immunofluorescence for ITPKA shows expression along EC nuclei, cell borders, and within the holes of the IEL. No staining was evident in the SMC. C, Proposed interaction model between Calr (gray) and ITPKA (blue). The ITPKA mimetic peptide is in red (Sequence: TAT tag-PEAPTEEEHA). D, Peptide blocking the interaction between Calr and ITPKA significantly blunted constriction to PE (Scrambled $n=5$ arteries, ITPKA $n=5$ arteries). E, Peptide had no significant effect on CCh dilation (Scrambled $n=4$ arteries, ITPKA $n=3$ arteries). 
FIGURE 38. KIF18B INTERACTS WITH CALR AT THE MEJ BUT HAS NO EFFECT ON PE CONSTRICTION
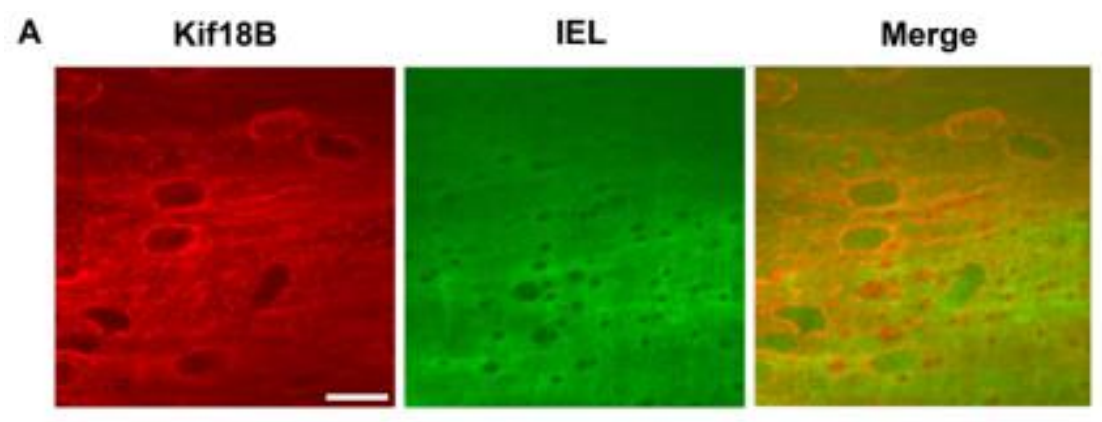

B

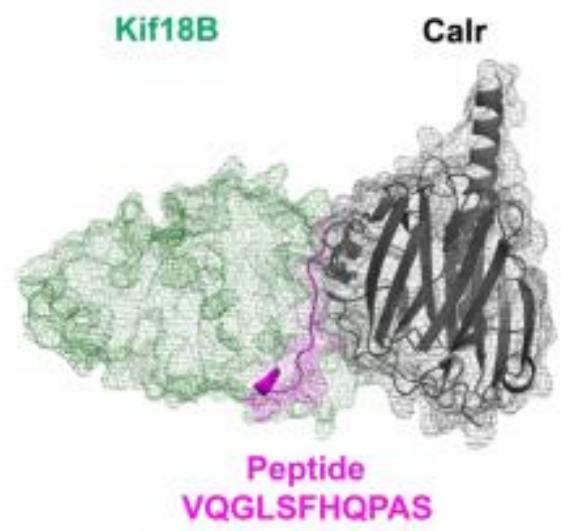

C

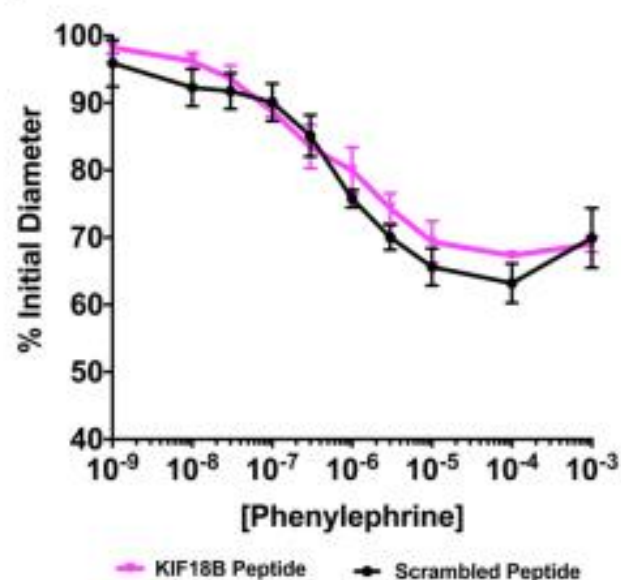


Figure 38. Kif18B interacts with Calr at the MEJ but has no effect on PE constriction. A, Expression of Kif18B (red) in the IEL (green) holes of third order mesenteric arteries. Scale bar $=20 \mu \mathrm{m} . \mathbf{B}$, Proposed interaction model between Calr (gray) and Kif18B (green). The Kif18A mimetic peptide is in fuschia (Sequence: TAT tag-VQGLSFHQPAS). C, There is no difference in constriction to PE with the peptide that blocks the Calr-Kif18B interaction (Scrambled $n=5$ arteries, Kif18b n= 3 arteries). 


\subsection{Discussion}

In approximately $30 \%$ of holes in the IEL, we observed all three well-established markers for ER, which indicates the presence of ER at the MEJ in some instances. This finding is consistent in the literature. ${ }^{13,26}$ However, the fact that Calr was found in nearly every hole in the IEL ( $80 \%)$ was unexpected and markedly different than the other ER markers. The permeabilization of arteries with digitonin and the inhibition of Calr transfer to holes in the IEL with brefeldin A further suggest that the pool of Calr found in nearly every hole in the IEL of the arteries was not ER-based and was indicative of a functionally unique pool of Calr. Our evidence in EC Calr $\Delta / \Delta$ mice confirm this; in third-order mesenteric arteries, the ECs no longer respond with increases in calcium after SMC stimulation (but there is no impairment in the response to muscarinic stimulation) and pressurized arteries are more reactive to $\mathrm{PE}$ (but there is no impairment in the response to muscarinic dilation). In addition, the mice have significantly increased blood pressure. This phenotype occurred despite no evident change in global ER calcium in the ECs. The sum of the evidence appears to indicate that non-ER based Calr in the holes of the IEL of mesenteric arteries is an important, and unexpected, component to regulating negative feedback from smooth muscle to endothelium.

The finding that traditional ER markers are not expressed in the majority of IEL holes was somewhat surprising. Previous research using en face immunofluorescence has shown calnexin in mouse mesenteric arteries is present in IEL holes ${ }^{19}$, but there was no quantification or multiple fields of view to know if the proportion of expression in IEL holes was similar to our results. While the ER is a continuous compartment, it could be possible that calnexin and ERp29 are differentially segregated to areas of the ER separate 
from Calr. Furthermore, the ER is particularly dynamic and has smooth and rough regions, which we initially thought could be an explanation for the discrepancy in expression in the IEL holes. The data presented here now suggest there may also be a population of IEL holes that do not exhibit ER expression, which lends support to the conclusion that Calr may be mediating heterocellular signaling from outside the ER.

\section{Genetic deletion of EC Calr}

Because global Calr deletion in mice is embryonically lethal at E14.5 52 and there is sparse evidence on cell specific Calr knockout mice ${ }^{55,235}$, we have no direct comparison to our knockout efficiency in vivo. Other researchers have attempted to knock out Calr in ECs and achieved only $\sim 50 \%$ decreases in protein in vitro. ${ }^{236}$ Using the same Calr $\mathrm{fl} / \mathrm{fl}$ mouse (but without the Cre recombinase), Zimmerman et al reported a knock out efficiency of $60 \%$ in SMC transfected with Cre-recombinase IRES-GFP. ${ }^{235}$ Clark et al. noted "calreticulin proved to be an exceedingly stable protein" after their work on Calr in untreated HL-60 cells. ${ }^{237}$

The calreticulin loxP sites in our mice ensure that the exons 4-7 are deleted, which span from the end of the $\mathrm{N}$ domain and encompass the whole $\mathrm{P}$ domain of the protein. ${ }^{41}$ Calreticulin expression is ubiquitous in every cell type (albeit at different levels) except erythrocytes. In line with this, greater than $90 \%$ of cells are also positive for Calr in the mouse diaphragm. Approximately 30-40\% of these Calr+ cells were CD31+, indicating they were EC that had Calr. We saw a $40 \%$ reduction in the total number of EC that express Calr, but there was no evidence of EC that did not express Calr, even after investigation of the cells with high levels of yellow fluorescence (indicating they had compromised membrane integrity, data not shown). Thus, the mean 
fluorescent intensity of Calr signal in the CD31+, Calr+ cells was examined in diaphragms from EC Calr fl/fl and EC Calr $\Delta / \Delta$ and both groups exhibited similar levels of fluorescent signal. Apoptosis or necrosis of EC lacking Calr cannot be ruled out in EC Calr $\Delta / \Delta$, although increased resistance to apoptosis has been shown in staurosporine treated mouse embryonic fibroblasts with calreticulin deficiency. ${ }^{238}$ The lack of CD31+, Calr- cells may be due to experimental design or technique. It may be that something changes in these cells that results in loss of the cells during the digestion, filtering and centrifugation steps prior to flow cytometry analysis.

Despite different techniques (flow cytometry versus western blotting), organisms (mouse diaphragm versus human primary EC) and antibodies (fluorescently conjugated primary versus non conjugated primary, both from different suppliers), we consistently failed to accomplish more than 50\% knockout in our mouse model or human primary EC. Injecting mice at 6 weeks of age for 10 days (versus older ages or fewer days of injections) with peanut oil or tamoxifen (data not shown) and the combination of three siRNAs in cell culture at 48 hours gave better results in knockout efficiency, but still not to the level expected. The combination of siRNA at $5 \mathrm{nM}$ was just as efficient as $25 \mathrm{nM}$ at any timepoint (24, 48 or 72 hours) and prolonging the harvest after transfection to 72 hours did not provide any additional benefit. It is likely we have achieved maximal knockout in our models and further research into Calr may explain why it is so difficult to achieve $>60 \%$ deletion in vivo and in vitro. Nevertheless, we see significant differences in physiology using a mouse model with significant but not complete deletion of EC Calr. This led us to propose a differential role for Calr at the MEJ versus Calr in the rest of the EC. 


\section{Physiological effects of EC Calr knockout}

An unexpected result was the differential response to $\mathrm{CCh}$ and $\mathrm{PE}$ in isolated mesenteric arteries. Both agonists increased calcium events at IEL holes, however EC Calr $\Delta / \Delta$ arteries were not able to further mobilize calcium in response to PE stimulation. This response is specifically localized to the IEL holes as PE does not stimulate calcium release in the EC monolayer. Smaller, more localized changes in ER calcium or compensatory increases in calcium influx (independent of TRPV4) in the EC cannot be ruled out in EC Calr $\Delta / \Delta$ arteries. However, our evidence does not show any significant differences in ER calcium within the mesenteric arteries or in human ECs transfected with GCaMP-ER. These findings correlate with other groups that have shown Calr deletion or knockdown does not affect $\mathrm{IP}_{3}$ mediated calcium stores ${ }^{49}$ Calreticulin deficient cells isolated from embryonic knockout mice do not have differences in cytosolic calcium or in response to ER calcium content estimation with thapsigargin. ${ }^{52}$ Furthermore, both resting and stimulated conditions did not change calcium levels in embryonic stem cells with or without calreticulin. ${ }^{49}$ The lack of difference between EC Calr fl/fl and $\Delta / \Delta$ in calcium signaling and dilation to $\mathrm{CCh}$ supports the finding that ER calcium is not globally changed in our model. In addition to dose response curves performed with $\mathrm{CCh}$, the dilation of pressurized arteries to a maximal dose of $\mathrm{CCh}$ was investigated. EC Calr fl/fl third order mesenteric arteries dilated on average $69 \%$ versus $59 \%$ in EC Calr $\Delta / \Delta$, but this difference was not significant. This is further evidence to show that EC Calr $\Delta / \Delta$ does not significantly affect $\mathrm{CCh}$ mediated calcium signaling and vasodilation in mesenteric arteries. However, altering EC calcium mobilization has been shown to increase constriction to $\mathrm{PE}^{26}$, which is consistent with our results in the isolated mesenteric arteries. Vascular responses such as 
basal myogenic tone at $80 \mathrm{mmHg}$ and activation of endothelial and MEJ localized calciumactivated potassium channels with NS309 indicated intact MEJ signaling and endothelial integrity in both groups. This confirms the differential response to PE is not simply due to an experimental artifact.

Blood pressure response in EC Calr knockout

There is a significant increase in mean arterial pressure in EC Calr $\Delta / \Delta$ post- Calr knockout. This effect may be due to prolonged deficits in the normal negative feedback that typically occurs at the MEJ with EC Calr knockout. The role of Calr in blood pressure regulation has never been investigated, but mice that overexpress Calr in the myocardium have increased systolic function but a lower ejection fraction and heart rate. ${ }^{63}$ Although blood pressure was not measured in this particular study, it seems likely that this overexpression of Calr would result in decreased blood pressure. These findings provide complementary evidence that increased Calr in cardiomyocytes has an opposite effect on blood pressure. In vascular SMC, Calr has been shown to destabilize the angiotensin receptor subtype $1\left(\mathrm{AT}_{1} \mathrm{R}\right) \mathrm{mRNA}^{58}{ }^{5}$ Because $\mathrm{AT}_{1} \mathrm{R}$ activation promotes vasoconstriction, vascular remodeling and oxidative stress, this is posited as a protective function of Calr, which could presumably promote lower blood pressure in vivo. If Calr has a similar function in the EC, this protective function would be lost and might also explain the observed higher blood pressure in EC Calr knockout mice.

Although there is an increase in the average daily blood pressure after 7 weeks of Calr $\Delta / \Delta$, acute measurement of blood pressure does not exhibit any significant changes in blood pressure or heart rate at night. This period is when the mice are most active and sympathetic nervous activity would presumably the highest. Injection of PE resulted in a 
significant decrease in diastolic blood pressure for the first 15 minutes following injection, which accounted for a decrease in MAP as well. The change in diastolic blood pressure cannot be explained by the stress of handling or injection, as the mice also were injected with a higher dose of PE in a separate experiment $(0.5 \mathrm{mg} / \mathrm{kg}$, data not shown) and did not exhibit any changes in the first 15 minutes following injection. The injections were done in their light cycle, when mice are less active. Perhaps if these had been performed during the dark cycle, different results would be obtained. There may also be compensatory responses to PE, such as cardiac mediated effects; heart rate tended to be lower in response, but was not significant. Repetition of these studies in the presence of a ganglionic blocker may tease out any differences in the vascular sympathetic response to PE.

The effect of PE on the heart has been debated. It may affect venous return to the heart, thereby increasing preload. It has been posited that the preload status of the heart will affect its response to PE; cardiac output will increase if the heart is preloaddependent and it will decrease if preload-independent. ${ }^{239}$ With Ang II administration in EC Calr fl/fl and $\Delta / \Delta$ mice, the only significant difference was within the first 24 hours of Ang II; EC Calr fl/fl mice significantly decreased their heart rate approximately 125bpm, presumably as a compensation for the approximately $15 \mathrm{mmHg}$ increase in blood pressure. However, EC Calr $\Delta / \Delta$ did not significantly adjust their heart rate as it only dipped approximately $25 \mathrm{bpm}$ on average. After 24 hours, this disparity in heart rate disappeared. 


\section{Potential roles for Calr in the EC of resistance arteries}

A possible explanation for our results is the role of non-ER based Calr as a mediator of integrin-based cell adhesion. There is strong evidence that indicates there is a calciumdependent interaction between Calr and the cytoplasmic tail of alpha integrin ${ }^{51,240}$. Calr downregulation in cells decreases their adhesive capacity and migration ${ }^{49,}$ 53, 236, 241 and some of these effects may be mediated by c-Src and calcium-calmodulin kinase II. ${ }^{242,243}$ Overexpression studies have also shown alterations in adhesion. ${ }^{64}$ This would be important in terms of resistance arteries as the $\mathrm{EC}$ and $\mathrm{MEJ}$ must be coupled to the SMC in order to receive the PE signal. ${ }^{244}$ Knockout of Calr has been shown to inhibit attachment to the extracellular matrix ${ }^{51}$ and downregulate cell adhesion-related genes. ${ }^{245}$ One of the upregulated genes was Notch1, which has been shown to induce SMC adhesion to the EC basement membrane via integrin signaling. ${ }^{246}$ Our results show no changes in IEL hole number in our arteries, but elucidation of adhesion mechanisms could be more sophisticated than simply changing the hole formation. Area of the holes did significantly decrease in EC Calr $\Delta / \Delta$ mesenteric arteries (data not shown) but it is still unclear what implications that might have on heterocellular communication. Surprisingly, cell adhesion at the MEJ, in addition to integrin expression and function has not been characterized. This is an intriguing future direction of exploration, particularly in the context of resistance artery function and control of blood pressure.

Beyond its role in cell adhesion and migration, calreticulin can be a major ER chaperone, key in maintaining proper protein folding, a calcium-dependent process. ${ }^{247}$ The knockdown of calreticulin protein has been shown by multiple groups to induce ER stress. ${ }^{67}, 248,249$ There is evidence that ER stress via tunicamycin causes endothelial 
dysfunction by decreased eNOS phosophorylation. ${ }^{250}$ Furthermore, ER stress inhibition in angiotensin II induced hypertension could improve vascular reactivity in mesenteric arteries. ${ }^{251}$ We cannot exclude the possible role of ER stress in our mouse model, especially in polarization of calcium responses and reactivity. However, we note several observations in the EC that lead us to conclude that uncontrolled ER stress may not be occurring in our model. These observations include the intact $\mathrm{CCh}$ calcium responses and vasodilation, as well as the undiminished responses to calcium-activated potassium channels with NS309. In both cases, significant ER stress would be expected to alter these fundamental vascular responses. Based on the evidence of calreticulin localization in the MEJ, it is unlikely that Calr is acting as an ER chaperone at the MEJ. In the EC monolayer, this may be more likely as we see a greater interaction with GRP78/BiP there versus the MEJ in vitro. It is also possible that our induction of calreticulin deletion post development (at 6 weeks of age) and without a concomitant pathological situation does not induce an ER stress response.

Within the ER, a major function of Calr is to properly fold proteins as part of the calreticulin-calnexin cycle. Calreticulin knockout has been shown to lead to an accumulation of misfolded proteins, thereby triggering the unfolded protein response to mitigate the problem. Binding immunoglobulin protein $(\mathrm{BiP}) / \mathrm{Glucose}$ regulated protein 78 is also a major ER chaperone that has increased expression in response to ER stress. BiP binds to ER stress sensors in normal physiological situations and disassociates during induction of ER stress with thapsigargin or DTT. This process is reversible if the ER stress is not maintained. ${ }^{252}$ For these reasons, we looked at BiP expression in response to EC Calr knockout in vitro and in vivo. We found a significant increase in BiP in the aorta 
of EC Calr $\Delta / \Delta$ and an increase in BiP in EC that had Calr siRNA. However, large vessels such as aortas have few, if any, MEJ ${ }^{4}$ and cultured EC are unable to form MEJ under standard culture conditions in a dish or flask. We do not have evidence for BiP expression in arteries with MEJ or the VCCC, but these findings suggest that in EC where MEJ do not form, Calr knockout increases BiP expression. Looking at brown adipose tissue, a highly vascularized organ that contains a more heterogeneous cellular population, we do not see differences in BiP expression. While this data is not comprehensive, taken together with the results that suggest different functional pools of Calr, EC monolayer Calr seems to be more important for regulation of ER protein folding and less involved in calcium signaling.

\section{Subcellular localization of Calr}

Calr has been reported in a number of subcellular compartments beyond the ER depending on the cell type, pathological condition and context. ${ }^{47}$ Our data demonstrate differences between Calr in the EC monolayer, which is perinuclear with typical ER-like staining, and the IEL holes, which have strong punctate staining. When the arteries are permeabilized with digitonin and not Triton, the typical EC monolayer staining is not visible while the MEJ Calr remains very similar. Because digitonin is a mild, non-ionic detergent that preferentially interacts with cholesterol, it does not permeabilize intracellular membranes such as the mitochondrial, lysosomal, nuclear or importantly, the ER membrane. ${ }^{231,232}$ Some evidence suggests calreticulin is currently the only ER-based mammalian protein able to retrotranslocate out of the ER independently of a protein degradation signal. ${ }^{233}$ Exactly how Calr is mediating calcium signaling outside of the ER at the MEJ may become evident by looking at downstream signaling targets. 
Signaling targets of EC Calr

Using mouse thoracic aortas, we looked at IP3R1 and SERCA2 mRNA to see if EC Calr deletion changed ER localized calcium channel expression. This allowed for us to use one artery per mouse instead of pooling tissues from different mice together, which has to be done when using mesenteric arteries. Calreticulin can interact with SERCA2b via its $\mathrm{P}$ domain containing a high capacity, low affinity calcium binding region and Calr overexpression inhibits repetitive calcium waves ${ }^{65}$, a finding that correlates with the high expression of Calr combined with the rapid and localized calcium dynamics noted at the MEJ. Calreticulin deficient mouse embryonic fibroblasts have decreased protein expression of the IP3R, albeit without any effect on function. ${ }^{42}$ In the thoracic aorta, we did not see differences in SERCA2 or IP3R mRNA expression. There could be differential regulation of these receptors in small mesenteric arteries so we cannot fully rule out that EC Calr $\Delta / \Delta$ does not change IP3R or SERCA2 expression, but given the results in the aorta it is unlikely. Other groups have reported that Calr deletion mutants did not affect SERCA2b expression. ${ }^{42,}{ }^{65}$ Pecam, a marker of EC, tended to be elevated in EC Calr $\Delta / \Delta$ aorta, but it was not significant ( $\mathrm{p}$ value $=0.3$ ). Thus, we cannot explain the changes in calcium signaling with changes the expression of these particular receptors or change in EC expression.

In mesenteric arteries, it may be that the activity of calcium-activated potassium channels (IK and SK) is diminished, as inhibition of IK and SK channels has also been shown to increase constriction to PE. ${ }^{26,253}$ Furthermore, mice lacking these channels in the EC also have increases in mean arterial pressure. ${ }^{254,255}$ These channels are strategically localized in and around IEL holes ${ }^{73}$, so it is plausible their activity may be detrimentally 
affected by EC Calr knockout. We do not see differences in dilation to NS309, an activator of the channels ${ }^{256}$, but this activation does not fully depend on increases in EC cytosolic calcium. The failure to increase calcium signals in response to PE with the EC Calr knockout could result in decreased activation of IK and SK, thus providing another mechanism for the increased constriction to PE and increased MAP.

Effect of aging on EC Calr in MEJ calcium signaling

In Figures 34 and 35, we showed that with age, (i) EC Calr $\Delta / \Delta$ are still able to significantly increase calcium events in response to CCh, (ii) EC Calr fl/fl retain their ability to increase calcium events to PE, (iii) EC Calr fl/fl mice increase calcium events three times as much as EC Calr $\Delta / \Delta$ to $\mathrm{CCh}$ and $\mathrm{PE}$, and (iv) the EC Calr $\Delta / \Delta$ remain unable to significantly mobilize calcium events to PE. When we compare young and aged mice within the same genotype, we see (i) aging decreases Calr expression in the EC Calr fl/fl thoracic aorta, (ii) aging significantly decreases calcium events at baseline in EC Calr $\Delta / \Delta$, but not in control mice, (iii) aging significantly decreases CCh stimulated calcium events in EC Calr $\Delta / \Delta$, but not in control mice.

The most striking difference when comparing young EC Calr fl/fl and EC Calr $\Delta / \Delta$ mice was the differential response to PE but not $\mathrm{CCh}$. However, when both groups of mice are aged, there is still a significant increase in calcium events in the EC Calr $\Delta / \Delta$ $(50 \%)$ but the EC Calr fl/fl arteries respond with $150 \%$ more calcium events in response to $\mathrm{CCh}$. This corresponds with other research involving endothelial tubes (live blood vessels in which SMC have been removed) from aged mice, where muscarinic stimulation increased ER mediated calcium release in relation to EC tubes from young 
mice. ${ }^{257}$ This may occur as a compensatory mechanism to counteract endothelial dysfunction.

Aging seems to have a greater detrimental effect on EC Calr $\Delta / \Delta$ arterial calcium signaling as both the baseline and $\mathrm{CCh}$ response are decreased when compared to the young mice of the same genotype. The mechanism of this remains to be explored, but the findings suggest that the aged mouse lacking EC Calr is unable to compensate for any age-related changes in EC dysfunction or signaling. Phenylephrine mediated constriction in rat mesenteric arteries increases with age and we have already showed this is occurring in young EC Calr $\Delta / \Delta$, along with increasing blood pressure over time. Indeed, enhanced ER stress, reactive oxygen species and sympathetic activity with age could be occurring earlier in EC Calr $\Delta / \Delta$ mice than EC Calr fl/fl. Although we did not observe any differences in lifespan up to 80 weeks of age, it may be that EC Calr $\Delta / \Delta$ mice are a model of accelerated vascular aging as evidenced by their impaired calcium control. Calr protein-protein interactions at the MEJ

Calreticulin has been shown to interact with a number of proteins, both inside and outside of the ER. ${ }^{41}$ The data presented here suggest there may be a difference in the function and localization of Calr in the EC monolayer versus the MEJ. Using our VCCC in vitro MEJ model, Calr in the EC tends to interact with more ER lumenal proteins (i.e. GRP78/Bip) and more cytosolic or transmembrane proteins in the MEJ (thrombospondin

\section{1, ITPKA, Kif18B).}

Inositol trisphosphate kinase $\mathrm{A}$ is an interesting target, given its known functions in dendritic spines of neurons where it can phosphorylate $\mathrm{IP}_{3}$ to create inositol $1,3,4,5$ tetrakisphosphate $\left(\mathrm{IP}_{4}\right)$, effectively controlling $\mathrm{IP}_{3}$ signaling, in addition to bundling $\mathrm{F}$ - 
$\operatorname{actin}^{258}$ and regulating morphology of the spines. ${ }^{259,}{ }^{260}$ In dendritic spines, it has been shown to co-localize with calcium-calmodulin kinase $\mathrm{II}^{261}$, a protein that additionally regulates calcium signaling at the MEJ. ${ }^{13}$ It has ATP and inositol binding motifs and a calmodulin binding domain, thus it is an innately calcium sensitive protein. Low calcium inhibits kinase activity, while high calcium promotes ITPKA phosphorylation of $\mathrm{IP}_{3}$. Given these observations, it is not surprising that ITPKA is also localized near ER in dendritic spines and co-localizes with phalloidin, a marker of F-actin, which is another main component of the MEJ structure. ${ }^{262}$

Both ITPKA and Kif18b were expressed within HIEL and immunoprecipitated with Calr. However, only the interaction between ITPKA and Calr affected constriction to PE, while the Kif18b and Calr peptide had no effect on PE mediated constriction. Therefore, the Calr-ITPKA interaction at the MEJ can control heterocellular signaling in mesenteric arteries but has no effect on $\mathrm{CCh}$ mediated dilation. This interaction may be disrupted in EC Calr knockout mice and may be a mechanism whereby $\mathrm{IP}_{3}$ signaling is tightly regulated at the $\mathrm{MEJ}$.

Production of $\mathrm{IP}_{4}$ locally at the MEJ by ITPKA phosphorylation would be a way to spatially and temporally control the $\mathrm{IP}_{3}$ signaling that occurs there. The combination of $\mathrm{IP}_{3}$ and $\mathrm{IP}_{4}$ can activate calcium-activated potassium channels ${ }^{263}$ and $\mathrm{IP}_{4}$ itself has been shown to activate a calcium sensitive, calcium permeable channel in $\mathrm{ECs}^{264}$ (likely TRPV4), both of which are key in MEJ calcium signaling. It is still unclear specifically how the Calr-ITPKA interaction affects calcium signals at the MEJ and why using the peptide causes less constriction to PE while the EC Calr $\Delta / \Delta$ arteries exhibited more 
constriction to PE. Investigating ITPKA expression in the arteries of EC Calr $\Delta / \Delta$ is an important foundation for further work in this area.

In conclusion, we have found a differential subcellular localization and potential role for MEJ Calr versus the EC monolayer Calr in small arteries that influences physiological blood pressure control. The data suggests that modulating Calr expression or its protein interactions outside of the ER at the MEJ may prove to be a viable target for antihypertensive therapy. 
CHAPTER 7. FUTURE DIRECTIONS 
The exact cellular location of MEJ Calr is still unclear, although we have evidence to suggest it is not ER based, it could be cytosolic or on the cell surface. Immunofluorescence of the EC and MEJ with no permeabilization would show only cell surface Calr, thus being able to determine if punctate staining at the MEJ remains. Calreticulin has been shown to travel to the plasma membrane via vesicles, which could explain the decrease seen in the HIEL with Brefeldin A. Furthermore, Calr expression at the MEJ via immuno electron microscopy in the EC Calr fl/fl and $\Delta / \Delta$ in combination with ER, cytosolic or plasma membrane markers could provide further evidence specifically where MEJ Calr is located before and after Calr knockout. Careful investigation of EC Calr $\Delta / \Delta$ arteries via electron microscopy could also directly reveal changes in MEJ formation, organelle localization and structure or other structural changes within the arterial wall.

We showed via immunofluorescence that calnexin expression appears similar to Calr in the EC monolayer, but not at the MEJ. However, we have not investigated calnexin expression in our EC Calr $\Delta / \Delta$ arteries. As a possible explanation for the preserved CCh response, it could be that calnexin expression is upregulated with Calr knockout. With aging, this calnexin upregulation may not be able to compensate for the loss of Calr and that would explain why aged mice no longer increase their calcium events to CCh. Calnexin has been shown to decrease $30-35 \%$ with age. ${ }^{265,266}$ If this is occurring, it would be important to dissect out the function of calnexin upregulation to understand whether it is a calcium binding or protein folding compensation, or both. Expression of Calr is very low in the adult heart, but post-natal upregulation of Calr significantly decreases connexin 43 levels and phosphorylation in the heart, 
affecting cellular communication. ${ }^{62}$ This provides an intriguing parallel to the MEJ because Cx43 is highly enriched at the MEJ versus the EC and SMC while other Cx are not. ${ }^{11}$ The expression and function of any $\mathrm{Cx}$, including $\mathrm{Cx} 43$, has not been investigated with EC Calr knockout, which could affect heterocellular signaling originating in the SMC. Changes in connexin expression or phosphorylation at the MEJ could affect calcium and/or $\mathrm{IP}_{3}$ movement from $\mathrm{SMC}$ to the $\mathrm{MEJ}$ and account for the decreased calcium signals seen in EC Calr $\Delta / \Delta$ arteries.

Another potential future direction would be expounding on the acute cardiac effects of EC Calr knockout. At $0.1 \mathrm{mg} / \mathrm{kg}$ dose of PE, the diastolic blood pressure response was significantly lower. To further understand why EC Calr $\Delta / \Delta$ affects diastolic blood pressure response to $\mathrm{PE}$, echocardiography could be performed on the mice to look for any differences in wall thickness, ventricle diameters and the end systolic and diastolic volumes. The other interesting piece of cardiac data indicated that the heart rate did not decrease in response to the first day of Ang II infusion in EC Calr $\Delta / \Delta$. This occurred despite Ang II causing equivalent increases in pressure. EC Calr fl/fl exhibited a significant drop in heart rate, presumably as a response to counteract the increased systemic resistance.

Although Calr clearly has effects on the developing and mature heart ${ }^{52,53,62,63}$, we would not necessarily predict that knockout of Calr after development in the endothelium would affect basic heart function, but cardiac microvascular EC can synthesize and secrete neuregulin-1, a growth factor that is protective against apoptosis of neighboring cardiomyocytes. ${ }^{267,268}$ Interestingly, EC neuregulin-1 mRNA expression is downregulated 3, 6 and 12 hours after in vitro administration of angiotensin II or PE. ${ }^{269}$ 
Neuregulin-1 downstream targets include SERCA2 and focal adhesion kinase, two proteins that Calr has also been shown to modulate. Furthermore, research on neuregulin1 in the nervous system has shown it can affect dendritic spines (projections from the dendrite, similar to MEJ with localized IP3R calcium release that promotes synaptic plasticity). More work would need to be done to look at microvascular EC in the heart and how EC Calr may be modulating the synthesis, secretion or signaling of neuregulin1. This could have broad implications for cardiovascular disease as recombinant Nrg1 is currently being studied for therapeutic effects post-myocardial infarction. In general, investigation of the cardiac microvascular EC and coronary artery function in EC Calr $\Delta / \Delta$ could prove to be very interesting, as alterations in EC calcium handing, protein folding and cell adhesion could be detrimental to cardiac function.

As noted in the discussion, cell adhesion at the MEJ has not been thoroughly investigated. Integrin activation and signaling is coupled to calcium influx and cell adhesion, two important processes that must occur at the MEJ. However, even the basal expression of integrins in this area of the vascular wall has not been specifically elucidated. Because cytosolic and cell surface Calr has been shown to activate integrins and affect calcium flux ${ }^{64,240,270}$, this may be another way to spatially and temporally control calcium signaling at the MEJ. Integrins rely on increased cytosolic calcium via $\mathrm{IP}_{3}$ mediated calcium release for activation by Calr. ${ }^{270}$ Thus, integrin signaling at the MEJ may be the missing link between Calr and calcium signaling at the MEJ. If Calr is on the cell surface at the MEJ, it could also be mediating disassembly of focal adhesions via thrombospondin-1 and/or focal adhesion kinase signaling. ${ }^{50}$ Furthermore, the high 
expression of PAI-1 at the MEJ points to the importance of matrix degradation and cell adhesion for MEJ formation and maintenance of signaling.

The "accelerated aging" phenotype in 75 week old EC Calr $\Delta / \Delta$ arteries is another promising area of future investigation. The effects of altered calcium signaling on vasoconstriction and dilation in the EC Calr $\Delta / \Delta$ mesenteric arteries have not been studied. Based on the decreased EC calcium responses to $\mathrm{CCh}$, endothelial dependent dilation is likely impaired. Because ER stress can increase with aging in EC, the use of TUDCA or PBA to inhibit ER stress may be able to alleviate the EC Calr $\Delta / \Delta$ impairments in calcium signaling. Investigating why the mice lose their response to $\mathrm{CCh}$ at a human equivalent of middle age while the EC Calr fl/fl mice have no evident alterations in calcium signaling should prove to be very interesting. Hydrogen peroxide, a byproduct of oxidative stress, is increased with aging and activates IK/SK channels ${ }^{271}$, which may not be occurring in the aged EC Calr $\Delta / \Delta$ endothelium. Further aging of the mice to 24 months and investigating calcium signaling would be interesting to see if that negates the differences seen at approximately 16 months in our aged model. Other groups have reported no change in baseline calcium signaling with age, using the fura 2 calcium indicator and EC tubes from larger mesenteric arteries of 24 month old mice, ${ }^{271}$ although the multiple variables make it difficult to draw parallel conclusions to our data.

All of the calcium signaling experiments were performed in the presence of TRPV4 inhibition, while we were unable to do this with the vasoreactivity experiments due to the presence of TRPV4 in smooth muscle cells. Unraveling the role of EC Calr on TRPV4 function in terms of calcium signaling could also be useful to further dissect out signaling pathways at the MEJ. 
Lastly, ITPKA has never been described in the EC before, let alone the MEJ, so even the presence of ITPKA in the vascular wall is novel and could be an interesting avenue of exploration. Now that we have established the localization, the function of ITPKA at the MEJ is an important next step. Because it has effects on both formation of dendritic spines and $\mathrm{IP}_{3}$ signaling in neurons, looking at $\mathrm{MEJ}$ formation via electron microscopy and calcium events in mesenteric HIEL from ITPKA -/- mice could begin to clarify the role of ITPKA in the vasculature. There is an ITPKA inhibitor that is commercially available which could be incubated with mesenteric arteries and then perform PE dose responses. We hypothesize that inhibiting the formation of $\mathrm{IP}_{4}$ Furthermore, coupling the calcium and vasoreactivity from aged EC Calr $\Delta / \Delta$ mice with an investigation of ITPKA activity and expression could prove to be an important link in the calcium signaling puzzle at the MEJ.

\subsection{Future Directions for Chapter 5}

This chapter provides a number of avenues for future research. Because all of the experiments were performed using the VCCC, the most important next step to translate the findings is to investigate the eNOS phosphorylation signaling in an intact artery between the EC and the MEJ. Our lab has shown previously that total eNOS is present in the EC and MEJ of intact mouse arteries, albeit in different ratios. ${ }^{11}$ The thoracodorsal (TDA) and cremaster artery have more MEJ eNOS than in the EC monolayer, while the coronary artery and mesenteric had roughly equivalent levels of eNOS. Another observation was that the TDA had 13 gold beads per $\mu \mathrm{m}^{2}$ at the MEJ, twice the amount seen at the mesenteric MEJ. ${ }^{11}$ In this study, phosphorylated eNOS (S1177) was only investigated in the TDA at basal conditions, but using immuno-electron microscopy, the 
location of phosphorylated eNOS could be easily distinguished with PE and BK stimulation. Additionally, the role of ER calcium versus extracellular calcium in the BK signaling pathway has not been specifically dissected out in the VCCC as we focused on the MEJ calcium and not the EC monolayer. Presumably, both would play a role in It is still unclear what is the direct signaling pathway that leads to eNOS phosphorylation at the MEJ. Calmodulin and AKT expression have not been determined at the MEJ, although presumably they are present. We showed that PKC activity increases at the MEJ in response to PE, but did not determine the exact PKC isoform at the MEJ, which would lend further avenues of exploration to the data presented here.

The differences in lipid composition between EC and SMC grown in the VCCC versus alone in a plastic flask was another interesting observation. While there are currently no studies to support this idea, experiments designed to modulate the lipid composition of co-cultured ECs and SMCs could lend support to the idea that lipids at the MEJ are essential for local NO signaling and heterocellular signaling in resistance arteries. Methyl- $\beta$-cyclodextrin can change lipid composition by depleting the lipid rafts within the PM of cholesterol. Our results show there is already less cholesterol at the MEJ, so it is unclear what effect, if any, this may have on the MEJ signaling. MEJ formation can be inhibited by coating the VCCC with collagen; we would predict that this would make the VCCC grown EC and SMC have similar lipid profiles to the EC and SMC grown on plastic dishes in a monolayer. Analysis of other lipid species at the MEJ beyond sphingolipids and basic phospholipids could be very informative, as we did not look at phosphoinositols or other components of lipid signaling. Phospholipase C isoforms are distributed throughout the mesenteric artery EC, including at the MEJ. ${ }^{272}$ 
Active phospholipase $\mathrm{C}$ at the MEJ could constituitively produce $\mathrm{IP}_{3}$ and $\mathrm{DAG}$ without needing receptor activation in the EC or SMC, which could explain the basal IP3R calcium events that occur at the MEJ.

Applying the knowledge gained from this data to a context such as vascular aging or hypertension could provide further insights to the role of MEJ signaling in pathophysiology. 


\section{CHAPTER 8. ACKNOWLEDGMENTS}

8.1 Acknowledgments for Chapter 2: Thank you to Anita Impagliazzo and Dr. Jerome Biwer for creation of Figure 1 and to my qualifier committee for helpful critiques of the manuscript.

8.2 Acknowledgments for Chapter 4: Thank you to Pooneh Bagher for her valuable feedback on the manuscript protocol. Christophe Lechauve contributed the western blots of PAI-1 and alpha globin. Sheri VanHoose of the University of Virginia (UVA) Research Histology Core contributed to the manuscript via the description of processing, embedding, and sectioning the VCCC. Stacey Guillot at the UVA Advanced Microscopy Facility performed processing, embedding, and sectioning of the artery for immuno transmission electron microscopy.

8.3 Acknowledgments for Chapter 5: Thank you to Adam Straub for generating the initial data for this paper and for the functional eNOS studies. Evan Taddeo and Brandon Kenwood isolated lipids from the VCCC fractions and collected the mass spectrometry data. Anita Impagliazzo created Figure 10. Brant Isakson assisted with image acquisition. 8.4 Acknowledgments for Chapter 6: Thank you to Adam Straub for the EM representative EM image and the UVA Advanced microscopy facility for processing and sectioning of the TDA for EM; Swapnil Sonkusare and Kwangseok Hong for their valuable expertise in obtaining and analysis of the calcium imaging data in addition to critical feedback on the project and manuscript; Miranda Good for assistance with blood pressure telemetry surgeries, vasoreactivity, flow cytometry and data analysis and interpretation; Nenja Krüger for assistance with Calr siRNA experiments and qPCR analysis; Brant Isakson for assistance with whole mount immunofluorescence image acquisition and 
quantification. Steven Keller (Rob's brother) and Linda Columbus generated the predicted models and peptide sequences for ITPKA and Kif18B. Gina Wimer performed the retroorbital bleeds. Sheri VanHoose at the UVA Research Histology Core processed, embedded, sectioned and stained mesenteric arteries in Figure 16. Robin Looft-Wilson, Neha Agrawal and Rahul Patel assisted with vasoreactivity experiments. 


\section{CHAPTER 9. REFERENCES}

1. Heberlein KR, Straub AC and Isakson BE. The myoendothelial junction: breaking through the matrix? Microcirculation. 2009;16:307-22.

2. Sandow SL, Senadheera S, Bertrand PP, Murphy TV and Tare M. Myoendothelial contacts, gap junctions, and microdomains: anatomical links to function?

Microcirculation. 2012;19:403-15.

3. Straub AC, Zeigler AC and Isakson BE. The myoendothelial junction: connections that deliver the message. Physiology (Bethesda). 2014;29:242-9.

4. Sandow SL, Tare M, Coleman HA, Hill CE and Parkington HC. Involvement of myoendothelial gap junctions in the actions of endothelium-derived hyperpolarizing factor. Circ Res. 2002;90:1108-13.

5. Moore $\mathrm{DH}$ and Ruska $\mathrm{H}$. The fine structure of capillaries and small arteries. J Biophys Biochem Cytol. 1957;3:457-62.

6. Parker F. An electron microscope study of coronary arteries. Am J Anat. 1958;103:247-73.

7. Heberlein KR, Straub AC, Best AK, Greyson MA, Looft-Wilson RC, Sharma PR, Meher $\mathrm{A}$, Leitinger $\mathrm{N}$ and Isakson BE. Plasminogen activator inhibitor-1 regulates myoendothelial junction formation. Circ Res. 2010;106:1092-102.

8. Ledoux J, Werner ME, Brayden JE and Nelson MT. Calcium-activated potassium channels and the regulation of vascular tone. Physiology (Bethesda). 2006;21:69-78.

9. Bagher P, Beleznai T, Kansui Y, Mitchell R, Garland CJ and Dora KA. Low intravascular pressure activates endothelial cell TRPV4 channels, local Ca2+ events, and IKCa channels, reducing arteriolar tone. Proc Natl Acad Sci U S A. 2012;109:18174-9. 10. Biwer LA, Taddeo EP, Kenwood BM, Hoehn KL, Straub AC and Isakson BE. Two functionally distinct pools of eNOS in endothelium are facilitated by myoendothelial junction lipid composition. Biochim Biophys Acta. 2016;1861:671-9.

11. Straub AC, Billaud M, Johnstone SR, Best AK, Yemen S, Dwyer ST, Looft-Wilson R, Lysiak JJ, Gaston B, Palmer L and Isakson BE. Compartmentalized connexin $43 \mathrm{~s}$ nitrosylation/denitrosylation regulates heterocellular communication in the vessel wall. Arterioscler Thromb Vasc Biol. 2011;31:399-407.

12. Sonkusare SK, Dalsgaard T, Bonev AD, Hill-Eubanks DC, Kotlikoff MI, Scott JD, Santana LF and Nelson MT. AKAP150-dependent cooperative TRPV4 channel gating is central to endothelium-dependent vasodilation and is disrupted in hypertension. Sci

Signal. 2014;7:ra66.

13. Toussaint F, Charbel $\mathrm{C}$, Blanchette $A$ and Ledoux J. CaMKII regulates intracellular $\mathrm{Ca}(2)(+)$ dynamics in native endothelial cells. Cell Calcium. 2015;58:275-85.

14. Isakson BE. Localized expression of an Ins $(1,4,5) \mathrm{P} 3$ receptor at the myoendothelial junction selectively regulates heterocellular $\mathrm{Ca} 2+$ communication. J Cell Sci. 2008;121:3664-73.

15. Biwer LA and Isakson BE. Endoplasmic reticulum-mediated signalling in cellular microdomains. Acta Physiol (Oxf). 2017;219:162-175. 
16. Sonkusare SK, Bonev AD, Ledoux J, Liedtke W, Kotlikoff MI, Heppner TJ, HillEubanks DC and Nelson MT. Elementary Ca2+ signals through endothelial TRPV4 channels regulate vascular function. Science. 2012;336:597-601.

17. Isakson BE and Duling BR. Heterocellular contact at the myoendothelial junction influences gap junction organization. Circ Res. 2005;97:44-51.

18. Isakson BE, Ramos SI and Duling BR. Ca2+ and inositol 1,4,5-trisphosphatemediated signaling across the myoendothelial junction. Circ Res. 2007;100:246-54. 19. Ledoux J, Taylor MS, Bonev AD, Hannah RM, Solodushko V, Shui B, Tallini Y, Kotlikoff MI and Nelson MT. Functional architecture of inositol 1,4,5-trisphosphate signaling in restricted spaces of myoendothelial projections. Proc Natl Acad Sci U S A. 2008;105:9627-32.

20. Straub AC, Butcher JT, Billaud M, Mutchler SM, Artamonov MV, Nguyen AT, Johnson T, Best AK, Miller MP, Palmer LA, Columbus L, Somlyo AV, Le TH and Isakson BE. Hemoglobin alpha/eNOS coupling at myoendothelial junctions is required for nitric oxide scavenging during vasoconstriction. Arterioscler Thromb Vasc Biol. 2014;34:2594600.

21. Kotlikoff MI. Genetically encoded Ca2+ indicators: using genetics and molecular design to understand complex physiology. J Physiol. 2007;578:55-67.

22. Sandow SL, Gzik DJ and Lee RM. Arterial internal elastic lamina holes: relationship to function? J Anat. 2009;214:258-66.

23. Dora KA, Doyle MP and Duling BR. Elevation of intracellular calcium in smooth muscle causes endothelial cell generation of NO in arterioles. Proc Natl Acad Sci U SA. 1997;94:6529-34.

24. Yashiro $\mathrm{Y}$ and Duling BR. Integrated $\mathrm{Ca}(2+)$ signaling between smooth muscle and endothelium of resistance vessels. Circ Res. 2000;87:1048-54.

25. Nausch LW, Bonev AD, Heppner TJ, Tallini Y, Kotlikoff MI and Nelson MT. Sympathetic nerve stimulation induces local endothelial $\mathrm{Ca} 2+$ signals to oppose vasoconstriction of mouse mesenteric arteries. Am J Physiol Heart Circ Physiol. 2012;302:H594-602.

26. Tran CH, Taylor MS, Plane F, Nagaraja S, Tsoukias NM, Solodushko V, Vigmond EJ, Furstenhaupt T, Brigdan M and Welsh DG. Endothelial Ca2+ wavelets and the induction of myoendothelial feedback. Am J Physiol Cell Physiol. 2012;302:C1226-42. 27. lino M. Biphasic Ca2+ dependence of inositol 1,4,5-trisphosphate-induced $\mathrm{Ca}$ release in smooth muscle cells of the guinea pig taenia caeci. J Gen Physiol. 1990;95:1103-22.

28. Hong KC, E.L.; Marziano, C; Sonkusare, SK. Smooth muscle alpha1-adrenergic receptor activation initiates a negative feedback mechanism that involves Ca2+ influx through endothelial TRPV4 channels. FASEB J. 2017;31.

29. Dickinson GD, Ellefsen KL, Dawson SP, Pearson JE and Parker I. Hindered cytoplasmic diffusion of inositol trisphosphate restricts its cellular range of action. Sci Signal. 2016;9:ra108.

30. Little TL, Xia J and Duling BR. Dye tracers define differential endothelial and smooth muscle coupling patterns within the arteriolar wall. Circ Res. 1995;76:498-504. 
31. Allbritton NL, Meyer T and Stryer L. Range of messenger action of calcium ion and inositol 1,4,5-trisphosphate. Science. 1992;258:1812-5.

32. Francis M, Waldrup JR, Qian X, Solodushko V, Meriwether J and Taylor MS. Functional Tuning of Intrinsic Endothelial Ca2+ Dynamics in Swine Coronary Arteries.

Circ Res. 2016;118:1078-90.

33. Qian X, Francis M, Solodushko V, Earley S and Taylor MS. Recruitment of dynamic endothelial $\mathrm{Ca} 2+$ signals by the TRPA1 channel activator AITC in rat cerebral arteries.

Microcirculation. 2013;20:138-48.

34. Edwards G, Feletou M and Weston AH. Endothelium-derived hyperpolarising factors and associated pathways: a synopsis. Pflugers Arch. 2010;459:863-79.

35. Edwards G, Dora KA, Gardener MJ, Garland CJ and Weston AH. K+ is an endothelium-derived hyperpolarizing factor in rat arteries. Nature. 1998;396:269-72.

36. Seki T, Goto K, Kiyohara K, Kansui Y, Murakami N, Haga Y, Ohtsubo T, Matsumura $\mathrm{K}$ and Kitazono T. Downregulation of Endothelial Transient Receptor Potential Vanilloid

Type 4 Channel and Small-Conductance of Ca2+-Activated K+ Channels Underpins Impaired Endothelium-Dependent Hyperpolarization in Hypertension. Hypertension. 2017;69:143-153.

37. Hong KC, E.L.; Marziano, C; Sonkusare, SK. Loss of local Ca2+ signaling networks in the endothelium in diet-induced obesity. FASEB J. 2016;30.

38. Straub AC, Lohman AW, Billaud M, Johnstone SR, Dwyer ST, Lee MY, Bortz PS, Best AK, Columbus L, Gaston B and Isakson BE. Endothelial cell expression of haemoglobin alpha regulates nitric oxide signalling. Nature. 2012;491:473-7.

39. Ostwald TJ and MacLennan DH. Isolation of a high affinity calcium-binding protein from sarcoplasmic reticulum. J Biol Chem. 1974;249:974-9.

40. Fliegel L, Burns K, MacLennan DH, Reithmeier RA and Michalak M. Molecular cloning of the high affinity calcium-binding protein (calreticulin) of skeletal muscle sarcoplasmic reticulum. J Biol Chem. 1989;264:21522-8.

41. Michalak M, Corbett EF, Mesaeli N, Nakamura K and Opas M. Calreticulin: one protein, one gene, many functions. Biochem J. 1999;344 Pt 2:281-92.

42. Nakamura K, Zuppini A, Arnaudeau S, Lynch J, Ahsan I, Krause R, Papp S, De Smedt H, Parys JB, Muller-Esterl W, Lew DP, Krause KH, Demaurex N, Opas M and Michalak M. Functional specialization of calreticulin domains. J Cell Biol. 2001;154:96172.

43. Pike SE, Yao L, Jones KD, Cherney B, Appella E, Sakaguchi K, Nakhasi H, TeruyaFeldstein J, Wirth P, Gupta G and Tosato G. Vasostatin, a calreticulin fragment, inhibits angiogenesis and suppresses tumor growth. J Exp Med. 1998;188:2349-56.

44. Klampfl T, Gisslinger $H$, Harutyunyan AS, Nivarthi $H$, Rumi E, Milosevic JD, Them

NC, Berg T, Gisslinger B, Pietra D, Chen D, Vladimer GI, Bagienski K, Milanesi C, Casetti IC, Sant'Antonio E, Ferretti V, Elena C, Schischlik F, Cleary C, Six M, Schalling M, Schonegger A, Bock C, Malcovati L, Pascutto C, Superti-Furga G, Cazzola M and Kralovics R. Somatic mutations of calreticulin in myeloproliferative neoplasms. $N$ Engl J Med. 2013;369:2379-90.

45. Nangalia J, Massie CE, Baxter EJ, Nice FL, Gundem G, Wedge DC, Avezov E, Li J, Kollmann K, Kent DG, Aziz A, Godfrey AL, Hinton J, Martincorena I, Van Loo P, Jones AV, 
Guglielmelli P, Tarpey P, Harding HP, Fitzpatrick JD, Goudie CT, Ortmann CA, Loughran SJ, Raine K, Jones DR, Butler AP, Teague JW, O'Meara S, McLaren S, Bianchi M, Silber Y, Dimitropoulou D, Bloxham D, Mudie L, Maddison M, Robinson B, Keohane C, Maclean C, Hill K, Orchard K, Tauro S, Du MQ, Greaves M, Bowen D, Huntly BJ, Harrison CN, Cross NC, Ron D, Vannucchi AM, Papaemmanuil E, Campbell PJ and Green AR. Somatic CALR mutations in myeloproliferative neoplasms with nonmutated JAK2. N Engl J Med. 2013;369:2391-405.

46. Guglielmelli P, Rotunno G, Fanelli T, Pacilli A, Brogi G, Calabresi L, Pancrazzi A and Vannucchi AM. Validation of the differential prognostic impact of type 1/type 1-like versus type 2/type 2-like CALR mutations in myelofibrosis. Blood Cancer J. 2015;5:e360.

47. Gold LI, Eggleton P, Sweetwyne MT, Van Duyn LB, Greives MR, Naylor SM, Michalak $M$ and Murphy-Ullrich JE. Calreticulin: non-endoplasmic reticulum functions in physiology and disease. FASEB J. 2010;24:665-83.

48. Wiersma VR, Michalak M, Abdullah TM, Bremer E and Eggleton P. Mechanisms of Translocation of ER Chaperones to the Cell Surface and Immunomodulatory Roles in Cancer and Autoimmunity. Front Oncol. 2015;5:7.

49. Coppolino MG, Woodside MJ, Demaurex N, Grinstein S, St-Arnaud R and Dedhar

S. Calreticulin is essential for integrin-mediated calcium signalling and cell adhesion. Nature. 1997;386:843-7.

50. Goicoechea S, Orr AW, Pallero MA, Eggleton P and Murphy-Ullrich JE. Thrombospondin mediates focal adhesion disassembly through interactions with cell surface calreticulin. J Biol Chem. 2000;275:36358-68.

51. Leung-Hagesteijn CY, Milankov K, Michalak M, Wilkins J and Dedhar S. Cell attachment to extracellular matrix substrates is inhibited upon downregulation of expression of calreticulin, an intracellular integrin alpha-subunit-binding protein. $J$ Cell Sci. 1994;107 ( Pt 3):589-600.

52. Mesaeli N, Nakamura K, Zvaritch E, Dickie P, Dziak E, Krause KH, Opas M, MacLennan DH and Michalak M. Calreticulin is essential for cardiac development. J Cell Biol. 1999;144:857-68.

53. Rauch F, Prud'homme J, Arabian A, Dedhar S and St-Arnaud R. Heart, brain, and body wall defects in mice lacking calreticulin. Exp Cell Res. 2000;256:105-11.

54. Park BJ, Lee DG, Yu JR, Jung SK, Choi K, Lee J, Lee J, Kim YS, Lee JI, Kwon JY, Lee J, Singson A, Song WK, Eom SH, Park CS, Kim DH, Bandyopadhyay J and Ahnn J.

Calreticulin, a calcium-binding molecular chaperone, is required for stress response and fertility in Caenorhabditis elegans. Mol Biol Cell. 2001;12:2835-45.

55. Tokuhiro K, Satouh Y, Nozawa K, Isotani A, Fujihara Y, Hirashima Y, Matsumura H, Takumi K, Miyano T, Okabe M, Benham AM and Ikawa M. Calreticulin is required for development of the cumulus oocyte complex and female fertility. Sci Rep. 2015;5:14254.

56. Burns K, Duggan B, Atkinson EA, Famulski KS, Nemer M, Bleackley RC and Michalak M. Modulation of gene expression by calreticulin binding to the glucocorticoid receptor. Nature. 1994;367:476-80.

57. Michalak M, Burns K, Andrin C, Mesaeli N, Jass GH, Busaan JL and Opas M. Endoplasmic reticulum form of calreticulin modulates glucocorticoid-sensitive gene expression. J Biol Chem. 1996;271:29436-45. 
58. Nickenig G, Michaelsen F, Muller C, Berger A, Vogel T, Sachinidis A, Vetter H and Bohm M. Destabilization of AT(1) receptor mRNA by calreticulin. Circ Res. 2002;90:53-8.

59. Saito Y, Ihara Y, Leach MR, Cohen-Doyle MF and Williams DB. Calreticulin functions in vitro as a molecular chaperone for both glycosylated and non-glycosylated proteins. EMBO J. 1999;18:6718-29.

60. Mery L, Mesaeli N, Michalak M, Opas M, Lew DP and Krause KH. Overexpression of calreticulin increases intracellular $\mathrm{Ca} 2+$ storage and decreases store-operated $\mathrm{Ca} 2+$ influx. J Biol Chem. 1996;271:9332-9.

61. Bastianutto C, Clementi E, Codazzi F, Podini P, De Giorgi F, Rizzuto R, Meldolesi J and Pozzan T. Overexpression of calreticulin increases the Ca2+ capacity of rapidly exchanging $\mathrm{Ca} 2+$ stores and reveals aspects of their lumenal microenvironment and function. J Cell Biol. 1995;130:847-55.

62. Lee D, Oka T, Hunter B, Robinson A, Papp S, Nakamura K, Srisakuldee W, Nickel BE, Light PE, Dyck JR, Lopaschuk GD, Kardami E, Opas M and Michalak M. Calreticulin induces dilated cardiomyopathy. PLoS One. 2013;8:e56387.

63. Nakamura K, Robertson M, Liu G, Dickie P, Nakamura K, Guo JQ, Duff HJ, Opas M, Kavanagh $\mathrm{K}$ and Michalak $\mathrm{M}$. Complete heart block and sudden death in mice overexpressing calreticulin. J Clin Invest. 2001;107:1245-53.

64. Ihara $Y$, Inai $Y$ and Ikezaki M. Alteration of integrin-dependent adhesion and signaling in EMT-like MDCK cells established through overexpression of calreticulin. $J$ Cell Biochem. 2011;112:2518-28.

65. Camacho $\mathrm{P}$ and Lechleiter JD. Calreticulin inhibits repetitive intracellular Ca2+ waves. Cell. 1995;82:765-71.

66. Roderick HL, Lechleiter JD and Camacho P. Cytosolic phosphorylation of calnexin controls intracellular $\mathrm{Ca}(2+)$ oscillations via an interaction with SERCA2b. J Cell Biol. 2000;149:1235-48.

67. Knee R, Ahsan I, Mesaeli N, Kaufman RJ and Michalak M. Compromised calnexin function in calreticulin-deficient cells. Biochem Biophys Res Commun. 2003;304:661-6.

68. Park MK, Petersen $\mathrm{OH}$ and Tepikin AV. The endoplasmic reticulum as one continuous $\mathrm{Ca}(2+)$ pool: visualization of rapid $\mathrm{Ca}(2+)$ movements and equilibration. The EMBO journal. 2000;19:5729-39.

69. Wu X and Bers DM. Sarcoplasmic reticulum and nuclear envelope are one highly interconnected $\mathrm{Ca} 2+$ store throughout cardiac myocyte. Circulation research. 2006;99:283-91.

70. Palade GE. The endoplasmic reticulum. The Journal of biophysical and biochemical cytology. 1956;2:85-98.

71. van Anken E, Romijn EP, Maggioni C, Mezghrani A, Sitia R, Braakman I and Heck AJ. Sequential waves of functionally related proteins are expressed when $B$ cells prepare for antibody secretion. Immunity. 2003;18:243-53.

72. Lee $C$ and Chen LB. Dynamic behavior of endoplasmic reticulum in living cells. Cell. 1988;54:37-46.

73. Billaud M, Lohman AW, Johnstone SR, Biwer LA, Mutchler S and Isakson BE. Regulation of cellular communication by signaling microdomains in the blood vessel wall. Pharmacol Rev. 2014;66:513-69. 
74. Caceres A, Banker G, Steward O, Binder L and Payne M. MAP2 is localized to the dendrites of hippocampal neurons which develop in culture. Brain research.

1984;315:314-8.

75. Cerqua C, Anesti V, Pyakurel A, Liu D, Naon D, Wiche G, Baffa R, Dimmer KS and Scorrano L. Trichoplein/mitostatin regulates endoplasmic reticulum-mitochondria juxtaposition. EMBO reports. 2010;11:854-60.

76. Bourne JN and Harris KM. Balancing structure and function at hippocampal dendritic spines. Annual review of neuroscience. 2008;31:47-67.

77. Wang SQ, Song LS, Lakatta EG and Cheng H. Ca2+ signalling between single Ltype Ca2+ channels and ryanodine receptors in heart cells. Nature. 2001;410:592-6.

78. Voeltz GK, Prinz WA, Shibata Y, Rist JM and Rapoport TA. A class of membrane proteins shaping the tubular endoplasmic reticulum. Cell. 2006;124:573-86.

79. Terasaki M, Shemesh T, Kasthuri N, Klemm RW, Schalek R, Hayworth KJ, Hand AR, Yankova M, Huber G, Lichtman JW, Rapoport TA and Kozlov MM. Stacked endoplasmic reticulum sheets are connected by helicoidal membrane motifs. Cell. 2013;154:285-96.

80. Shibata Y, Voeltz GK and Rapoport TA. Rough sheets and smooth tubules. Cell. 2006;126:435-9.

81. Shibata Y, Shemesh T, Prinz WA, Palazzo AF, Kozlov MM and Rapoport TA. Mechanisms determining the morphology of the peripheral ER. Cell. 2010;143:774-88.

82. Hu J, Prinz WA and Rapoport TA. Weaving the web of ER tubules. Cell. 2011;147:1226-31.

83. Shemesh T, Klemm RW, Romano FB, Wang S, Vaughan J, Zhuang X, Tukachinsky $\mathrm{H}$, Kozlov MM and Rapoport TA. A model for the generation and interconversion of ER morphologies. Proceedings of the National Academy of Sciences of the United States of America. 2014;111:E5243-51.

84. Bjork S, Hurt CM, Ho VK and Angelotti T. REEPs are membrane shaping adapter proteins that modulate specific g protein-coupled receptor trafficking by affecting ER cargo capacity. PloS one. 2013;8:e76366.

85. Hu J, Shibata Y, Voss C, Shemesh T, Li Z, Coughlin M, Kozlov MM, Rapoport TA and Prinz WA. Membrane proteins of the endoplasmic reticulum induce high-curvature tubules. Science. 2008;319:1247-50.

86. Shibata Y, Voss C, Rist JM, Hu J, Rapoport TA, Prinz WA and Voeltz GK. The reticulon and DP1/Yop1p proteins form immobile oligomers in the tubular endoplasmic reticulum. The Journal of biological chemistry. 2008;283:18892-904.

87. Park SH and Blackstone $\mathrm{C}$. Further assembly required: construction and dynamics of the endoplasmic reticulum network. EMBO reports. 2010;11:515-21.

88. Hu J, Shibata Y, Zhu PP, Voss C, Rismanchi N, Prinz WA, Rapoport TA and Blackstone C. A class of dynamin-like GTPases involved in the generation of the tubular ER network. Cell. 2009;138:549-61.

89. Chen S, Novick $P$ and Ferro-Novick S. ER network formation requires a balance of the dynamin-like GTPase Sey1p and the Lunapark family member Lnp1p. Nature cell biology. 2012;14:707-16. 
90. Chen S, Desai T, McNew JA, Gerard P, Novick PJ and Ferro-Novick S. Lunapark stabilizes nascent three-way junctions in the endoplasmic reticulum. Proceedings of the National Academy of Sciences of the United States of America. 2015;112:418-23.

91. Yamamoto Y, Yoshida A, Miyazaki N, Iwasaki K and Sakisaka T. ArI6IP1 has the ability to shape the mammalian ER membrane in a reticulon-like fashion. The Biochemical journal. 2014;458:69-79.

92. Audhya A, Desai A and Oegema K. A role for Rab5 in structuring the endoplasmic reticulum. The Journal of cell biology. 2007;178:43-56.

93. Feiguin F, Ferreira A, Kosik KS and Caceres A. Kinesin-mediated organelle translocation revealed by specific cellular manipulations. The Journal of cell biology. 1994;127:1021-39.

94. Wozniak MJ, Bola B, Brownhill K, Yang YC, Levakova V and Allan VJ. Role of kinesin-1 and cytoplasmic dynein in endoplasmic reticulum movement in VERO cells. Journal of cell science. 2009;122:1979-89.

95. Bridgman PC. Myosin Va movements in normal and dilute-lethal axons provide support for a dual filament motor complex. The Journal of cell biology. 1999;146:104560.

96. Wagner W, Brenowitz SD and Hammer JA, 3rd. Myosin-Va transports the endoplasmic reticulum into the dendritic spines of Purkinje neurons. Nature cell biology. 2011;13:40-8.

97. Waterman-Storer CM and Salmon ED. Endoplasmic reticulum membrane tubules are distributed by microtubules in living cells using three distinct mechanisms. Current biology : CB. 1998;8:798-806.

98. Friedman JR, Webster BM, Mastronarde DN, Verhey KJ and Voeltz GK. ER sliding dynamics and ER-mitochondrial contacts occur on acetylated microtubules. The Journal of cell biology. 2010;190:363-75.

99. Grigoriev I, Gouveia SM, van der Vaart B, Demmers J, Smyth JT, Honnappa S, Splinter D, Steinmetz MO, Putney JW, Jr., Hoogenraad CC and Akhmanova A. STIM1 is a MT-plus-end-tracking protein involved in remodeling of the ER. Current biology : $C B$. 2008;18:177-82.

100. Klopfenstein DR, Kappeler F and Hauri HP. A novel direct interaction of endoplasmic reticulum with microtubules. The EMBO journal. 1998;17:6168-77.

101. Klopfenstein DR, Klumperman J, Lustig A, Kammerer RA, Oorschot V and Hauri HP. Subdomain-specific localization of CLIMP-63 (p63) in the endoplasmic reticulum is mediated by its luminal alpha-helical segment. The Journal of cell biology. 2001;153:1287-300.

102. Dreier $L$ and Rapoport TA. In vitro formation of the endoplasmic reticulum occurs independently of microtubules by a controlled fusion reaction. The Journal of cell biology. 2000;148:883-98.

103. Grayson TH, Haddock RE, Murray TP, Wojcikiewicz RJ and Hill CE. Inositol 1,4,5trisphosphate receptor subtypes are differentially distributed between smooth muscle and endothelial layers of rat arteries. Cell calcium. 2004;36:447-58. 
104. Lange $\mathrm{Y}$, Ye J, Rigney M and Steck TL. Regulation of endoplasmic reticulum cholesterol by plasma membrane cholesterol. Journal of lipid research. 1999;40:226470.

105. van Meer G, Voelker DR and Feigenson GW. Membrane lipids: where they are and how they behave. Nature reviews Molecular cell biology. 2008;9:112-24.

106. Zambrano F, Fleischer S and Fleischer B. Lipid composition of the Golgi apparatus of rat kidney and liver in comparison with other subcellular organelles. Biochimica et biophysica acta. 1975;380:357-69.

107. Ikonen E. Cellular cholesterol trafficking and compartmentalization. Nature reviews Molecular cell biology. 2008;9:125-38.

108. Feng B, Yao PM, Li Y, Devlin CM, Zhang D, Harding HP, Sweeney M, Rong JX, Kuriakose G, Fisher EA, Marks AR, Ron D and Tabas I. The endoplasmic reticulum is the site of cholesterol-induced cytotoxicity in macrophages. Nature cell biology. 2003;5:78192.

109. van Meer $\mathrm{G}$ and Lisman Q. Sphingolipid transport: rafts and translocators. The Journal of biological chemistry. 2002;277:25855-8.

110. Farese RV, Jr. and Walther TC. Lipid droplets finally get a little R-E-S-P-E-C-T. Cell. 2009;139:855-60.

111. Lev S. Nonvesicular lipid transfer from the endoplasmic reticulum. Cold Spring Harbor perspectives in biology. 2012;4.

112. Liscum L, Finer-Moore J, Stroud RM, Luskey KL, Brown MS and Goldstein JL. Domain structure of 3-hydroxy-3-methylglutaryl coenzyme A reductase, a glycoprotein of the endoplasmic reticulum. The Journal of biological chemistry. 1985;260:522-30.

113. Fagone P and Jackowski S. Membrane phospholipid synthesis and endoplasmic reticulum function. Journal of lipid research. 2009;50 Suppl:S311-6.

114. Loewen CJ, Roy A and Levine TP. A conserved ER targeting motif in three families of lipid binding proteins and in Opi1p binds VAP. The EMBO journal. 2003;22:2025-35.

115. Ponting CP and Aravind L. START: a lipid-binding domain in StAR, HD-ZIP and signalling proteins. Trends in biochemical sciences. 1999;24:130-2.

116. Kumagai K, Yasuda S, Okemoto K, Nishijima M, Kobayashi S and Hanada K. CERT mediates intermembrane transfer of various molecular species of ceramides. The Journal of biological chemistry. 2005;280:6488-95.

117. Hanada K, Kumagai K, Yasuda S, Miura Y, Kawano M, Fukasawa M and Nishijima M. Molecular machinery for non-vesicular trafficking of ceramide. Nature.

2003;426:803-9.

118. Levine T. Short-range intracellular trafficking of small molecules across endoplasmic reticulum junctions. Trends in cell biology. 2004;14:483-90.

119. Fukasawa M, Nishijima M and Hanada K. Genetic evidence for ATP-dependent endoplasmic reticulum-to-Golgi apparatus trafficking of ceramide for sphingomyelin synthesis in Chinese hamster ovary cells. The Journal of cell biology. 1999;144:673-85.

120. Cockcroft S. Phosphatidylinositol transfer proteins couple lipid transport to phosphoinositide synthesis. Seminars in cell \& developmental biology. 2001;12:183-91. 
121. Brown AJ, Sun L, Feramisco JD, Brown MS and Goldstein JL. Cholesterol addition to ER membranes alters conformation of SCAP, the SREBP escort protein that regulates cholesterol metabolism. Molecular cell. 2002;10:237-45.

122. Fryer LG, Jones B, Duncan EJ, Hutchison CE, Ozkan T, Williams PA, Alder O, Nieuwdorp M, Townley AK, Mensenkamp AR, Stephens DJ, Dallinga-Thie GM and Shoulders CC. The endoplasmic reticulum coat protein II transport machinery coordinates cellular lipid secretion and cholesterol biosynthesis. The Journal of biological chemistry. 2014;289:4244-61.

123. Espenshade PJ, Li WP and Yabe D. Sterols block binding of COPII proteins to SCAP, thereby controlling SCAP sorting in ER. Proceedings of the National Academy of Sciences of the United States of America. 2002;99:11694-9.

124. Yang T, Espenshade PJ, Wright ME, Yabe D, Gong Y, Aebersold R, Goldstein JL and Brown MS. Crucial step in cholesterol homeostasis: sterols promote binding of SCAP to INSIG-1, a membrane protein that facilitates retention of SREBPs in ER. Cell. 2002;110:489-500.

125. Yabe D, Brown MS and Goldstein JL. Insig-2, a second endoplasmic reticulum protein that binds SCAP and blocks export of sterol regulatory element-binding proteins. Proceedings of the National Academy of Sciences of the United States of America. 2002;99:12753-8.

126. Miyata S, Inoue J, Shimizu M and Sato R. Xanthohumol Improves Diet-induced Obesity and Fatty Liver by Suppressing Sterol Regulatory Element-binding Protein (SREBP) Activation. The Journal of biological chemistry. 2015;290:20565-79.

127. Sakai J, Rawson RB, Espenshade PJ, Cheng D, Seegmiller AC, Goldstein JL and Brown MS. Molecular identification of the sterol-regulated luminal protease that cleaves SREBPs and controls lipid composition of animal cells. Molecular cell. 1998;2:505-14. 128. Nohturfft A, DeBose-Boyd RA, Scheek S, Goldstein JL and Brown MS. Sterols regulate cycling of SREBP cleavage-activating protein (SCAP) between endoplasmic reticulum and Golgi. Proceedings of the National Academy of Sciences of the United States of America. 1999;96:11235-40.

129. Bartz R, Sun LP, Bisel B, Wei JH and Seemann J. Spatial separation of Golgi and ER during mitosis protects SREBP from unregulated activation. The EMBO journal. 2008;27:948-55.

130. Bezprozvanny I, Watras J and Ehrlich BE. Bell-shaped calcium-response curves of Ins $(1,4,5)$ P3- and calcium-gated channels from endoplasmic reticulum of cerebellum. Nature. 1991;351:751-4.

131. Montero M, Alvarez J, Scheenen WJ, Rizzuto R, Meldolesi J and Pozzan T. Ca2+ homeostasis in the endoplasmic reticulum: coexistence of high and low [Ca2+] subcompartments in intact HeLa cells. The Journal of cell biology. 1997;139:601-11. 132. Vermassen E, Parys JB and Mauger JP. Subcellular distribution of the inositol 1,4,5-trisphosphate receptors: functional relevance and molecular determinants. Biology of the cell / under the auspices of the European Cell Biology Organization. 2004;96:3-17.

133. Wojcikiewicz RJ. Type I, II, and III inositol 1,4,5-trisphosphate receptors are unequally susceptible to down-regulation and are expressed in markedly different 
proportions in different cell types. The Journal of biological chemistry. 1995;270:1167883.

134. Miyakawa T, Maeda A, Yamazawa T, Hirose K, Kurosaki T and lino M. Encoding of Ca2+ signals by differential expression of IP3 receptor subtypes. The EMBO journal. 1999;18:1303-8.

135. Smith IF, Wiltgen SM, Shuai J and Parker I. $\mathrm{Ca}(2+)$ puffs originate from preestablished stable clusters of inositol trisphosphate receptors. Science signaling. 2009;2:ra77.

136. Mitchell CA, Connolly TM and Majerus PW. Identification and isolation of a 75kDa inositol polyphosphate-5-phosphatase from human platelets. The Journal of biological chemistry. 1989;264:8873-7.

137. Hansen CA, Johanson RA, Williamson MT and Williamson JR. Purification and characterization of two types of soluble inositol phosphate 5-phosphomonoesterases from rat brain. The Journal of biological chemistry. 1987;262:17319-26.

138. Sims CE and Allbritton NL. Metabolism of inositol 1,4,5-trisphosphate and inositol 1,3,4,5-tetrakisphosphate by the oocytes of Xenopus laevis. The Journal of biological chemistry. 1998;273:4052-8.

139. Lanner JT, Georgiou DK, Joshi AD and Hamilton SL. Ryanodine receptors: structure, expression, molecular details, and function in calcium release. Cold Spring Harbor perspectives in biology. 2010;2:a003996.

140. Bezprozvanny I. Inositol (1,4,5)-trisphosphate receptors: functional properties, modulation, and role in calcium wave propagation. Society of General Physiologists series. 1996;51:75-86.

141. Cheng $\mathrm{H}$, Lederer WJ and Cannell MB. Calcium sparks: elementary events underlying excitation-contraction coupling in heart muscle. Science. 1993;262:740-4.

142. Nelson MT, Cheng H, Rubart M, Santana LF, Bonev AD, Knot HJ and Lederer WJ.

Relaxation of arterial smooth muscle by calcium sparks. Science. 1995;270:633-7. 143. Park MK, Choi YM, Kang YK and Petersen $\mathrm{OH}$. The endoplasmic reticulum as an integrator of multiple dendritic events. The Neuroscientist : a review journal bringing neurobiology, neurology and psychiatry. 2008;14:68-77.

144. Emptage N, Bliss TV and Fine A. Single synaptic events evoke NMDA receptormediated release of calcium from internal stores in hippocampal dendritic spines. Neuron. 1999;22:115-24.

145. Toussaint F, Charbel C, Blanchette A and Ledoux J. CaMKII regulates intracellular $\mathrm{Ca}(2+)$ dynamics in native endothelial cells. Cell calcium. 2015;58:275-85.

146. Heberlein KR, Han J, Straub AC, Best AK, Kaun C, Wojta J and Isakson BE. A novel mRNA binding protein complex promotes localized plasminogen activator inhibitor-1 accumulation at the myoendothelial junction. Arterioscler Thromb Vasc Biol. 2012;32:1271-9.

147. Takei K, Stukenbrok H, Metcalf A, Mignery GA, Sudhof TC, Volpe P and De Camilli $\mathrm{P}$. Ca2+ stores in Purkinje neurons: endoplasmic reticulum subcompartments demonstrated by the heterogeneous distribution of the InsP3 receptor, $\mathrm{Ca}(2+)$-ATPase, and calsequestrin. The Journal of neuroscience : the official journal of the Society for Neuroscience. 1992;12:489-505. 
148. Bare DJ, Kettlun CS, Liang M, Bers DM and Mignery GA. Cardiac type 2 inositol 1,4,5-trisphosphate receptor: interaction and modulation by calcium/calmodulindependent protein kinase II. The Journal of biological chemistry. 2005;280:15912-20. 149. Terentyev D, Viatchenko-Karpinski S, Vedamoorthyrao S, Oduru S, Gyorke I, Williams SC and Gyorke S. Protein protein interactions between triadin and calsequestrin are involved in modulation of sarcoplasmic reticulum calcium release in cardiac myocytes. The Journal of physiology. 2007;583:71-80.

150. Michalak M, Groenendyk J, Szabo E, Gold LI and Opas M. Calreticulin, a multiprocess calcium-buffering chaperone of the endoplasmic reticulum. Biochem J. 2009;417:651-66.

151. Biwer L, Straub A and Isakson B. Subcellular enrichment of calreticulin at myoendothelial junctions (664.2). The FASEB Journal. 2014;28:664.2.

152. Finch EA and Augustine GJ. Local calcium signalling by inositol-1,4,5trisphosphate in Purkinje cell dendrites. Nature. 1998;396:753-6.

153. Aiba A, Kano M, Chen C, Stanton ME, Fox GD, Herrup K, Zwingman TA and Tonegawa $S$. Deficient cerebellar long-term depression and impaired motor learning in mGluR1 mutant mice. Cell. 1994;79:377-88.

154. Khodakhah $\mathrm{K}$ and Armstrong CM. Induction of long-term depression and rebound potentiation by inositol trisphosphate in cerebellar Purkinje neurons. Proceedings of the National Academy of Sciences of the United States of America. 1997;94:14009-14.

155. Inoue $\mathrm{T}$, Kato K, Kohda K and Mikoshiba K. Type 1 inositol 1,4,5-trisphosphate

receptor is required for induction of long-term depression in cerebellar Purkinje neurons. The Journal of neuroscience : the official journal of the Society for Neuroscience. 1998;18:5366-73.

156. Kansui Y, Garland CJ and Dora KA. Enhanced spontaneous Ca2+ events in endothelial cells reflect signalling through myoendothelial gap junctions in pressurized mesenteric arteries. Cell Calcium. 2008;44:135-46.

157. Toulmay A and Prinz WA. Lipid transfer and signaling at organelle contact sites: the tip of the iceberg. Current opinion in cell biology. 2011;23:458-63.

158. Rizzuto R, Pinton P, Carrington W, Fay FS, Fogarty KE, Lifshitz LM, Tuft RA and

Pozzan T. Close contacts with the endoplasmic reticulum as determinants of mitochondrial Ca2+ responses. Science. 1998;280:1763-6.

159. Friedman JR, Lackner LL, West M, DiBenedetto JR, Nunnari J and Voeltz GK. ER tubules mark sites of mitochondrial division. Science. 2011;334:358-62.

160. Shiao YJ, Lupo $G$ and Vance JE. Evidence that phosphatidylserine is imported into mitochondria via a mitochondria-associated membrane and that the majority of mitochondrial phosphatidylethanolamine is derived from decarboxylation of phosphatidylserine. The Journal of biological chemistry. 1995;270:11190-8.

161. Olson ML, Chalmers S and McCarron JG. Mitochondrial Ca2+ uptake increases Ca2+ release from inositol 1,4,5-trisphosphate receptor clusters in smooth muscle cells. The Journal of biological chemistry. 2010;285:2040-50.

162. Vance JE. Phospholipid synthesis in a membrane fraction associated with mitochondria. The Journal of biological chemistry. 1990;265:7248-56. 
163. Stone SJ and Vance JE. Phosphatidylserine synthase- 1 and -2 are localized to mitochondria-associated membranes. The Journal of biological chemistry. 2000;275:34534-40.

164. de Brito OM and Scorrano L. Mitofusin 2 tethers endoplasmic reticulum to mitochondria. Nature. 2008;456:605-10.

165. Zampese E, Fasolato C, Kipanyula MJ, Bortolozzi M, Pozzan T and Pizzo P.

Presenilin 2 modulates endoplasmic reticulum (ER)-mitochondria interactions and $\mathrm{Ca} 2+$ cross-talk. Proceedings of the National Academy of Sciences of the United States of America. 2011;108:2777-82.

166. Carrasco $S$ and Meyer T. STIM proteins and the endoplasmic reticulum-plasma membrane junctions. Annual review of biochemistry. 2011;80:973-1000.

167. Hoth $M$ and Penner R. Depletion of intracellular calcium stores activates a calcium current in mast cells. Nature. 1992;355:353-6.

168. Park CY, Hoover PJ, Mullins FM, Bachhawat P, Covington ED, Raunser S, Walz T, Garcia KC, Dolmetsch RE and Lewis RS. STIM1 clusters and activates CRAC channels via direct binding of a cytosolic domain to Orai1. Cell. 2009;136:876-90.

169. Liou J, Kim ML, Heo WD, Jones JT, Myers JW, Ferrell JE, Jr. and Meyer T. STIM is a $\mathrm{Ca} 2+$ sensor essential for $\mathrm{Ca} 2+-$ store-depletion-triggered $\mathrm{Ca} 2+$ influx. Current biology : CB. 2005;15:1235-41.

170. Jozsef L, Tashiro K, Kuo A, Park E, Skoura A, Albinsson S, Rivera-Molina F, Harrison KD, Iwakiri Y, Toomre D and Sessa WC. Reticulon 4 Is Necessary for Endoplasmic Reticulum Tubulation, STIM1-Orai1 Coupling, and Store-operated Calcium Entry. The Journal of biological chemistry. 2014;289:9380-95.

171. Taddeo EP, Laker RC, Breen DS, Akhtar YN, Kenwood BM, Liao JA, Zhang M, Fazakerley DJ, Tomsig JL, Harris TE, Keller SR, Chow JD, Lynch KR, Chokki M, Molkentin JD, Turner N, James DE, Yan Z and Hoehn KL. Opening of the mitochondrial permeability transition pore links mitochondrial dysfunction to insulin resistance in skeletal muscle.

Molecular metabolism. 2014;3:124-34.

172. Kharel Y, Morris EA, Congdon MD, Thorpe SB, Tomsig JL, Santos WL and Lynch KR. Sphingosine Kinase 2 Inhibition and Blood Sphingosine 1-phosphate Levels. The Journal of pharmacology and experimental therapeutics. 2015.

173. Chow JD, Lawrence RT, Healy ME, Dominy JE, Liao JA, Breen DS, Byrne FL, Kenwood BM, Lackner C, Okutsu S, Mas VR, Caldwell SH, Tomsig JL, Cooney GJ, Puigserver PB, Turner N, James DE, Villen J and Hoehn KL. Genetic inhibition of hepatic acetyl-CoA carboxylase activity increases liver fat and alters global protein acetylation. Molecular metabolism. 2014;3:419-31.

174. Stuelsatz P, Keire P, Almuly R and Yablonka-Reuveni Z. A contemporary atlas of the mouse diaphragm: myogenicity, vascularity, and the Pax3 connection. J Histochem Cytochem. 2012;60:638-57.

175. Tovchigrechko A and Vakser IA. Development and testing of an automated approach to protein docking. Proteins. 2005;60:296-301.

176. Truskey GA. Endothelial Cell Vascular Smooth Muscle Cell Co-Culture Assay For High Throughput Screening Assays For Discovery of Anti-Angiogenesis Agents and Other Therapeutic Molecules. Int J High Throughput Screen. 2010;2010:171-181. 
177. Axel DI, Brehm BR, Wolburg-Buchholz K, Betz EL, Koveker G and Karsch KR. Induction of cell-rich and lipid-rich plaques in a transfilter coculture system with human vascular cells. J Vasc Res. 1996;33:327-39.

178. Hastings NE, Simmers MB, McDonald OG, Wamhoff BR and Blackman BR. Atherosclerosis-prone hemodynamics differentially regulates endothelial and smooth muscle cell phenotypes and promotes pro-inflammatory priming. Am J Physiol Cell Physiol. 2007;293:C1824-33.

179. Herzog DP, Dohle E, Bischoff I and Kirkpatrick CJ. Cell communication in a coculture system consisting of outgrowth endothelial cells and primary osteoblasts. Biomed Res Int. 2014;2014:320123.

180. Jacot JG and Wong JY. Endothelial injury induces vascular smooth muscle cell proliferation in highly localized regions of a direct contact co-culture system. Cell Biochem Biophys. 2008;52:37-46.

181. Scott-Drechsel D, Su Z, Hunter K, Li M, Shandas R and Tan W. A new flow coculture system for studying mechanobiology effects of pulse flow waves. Cytotechnology. 2012;64:649-66.

182. van Buul-Wortelboer MF, Brinkman HJ, Dingemans KP, de Groot PG, van Aken WG and van Mourik JA. Reconstitution of the vascular wall in vitro. A novel model to study interactions between endothelial and smooth muscle cells. Exp Cell Res. 1986;162:151-8.

183. Wilhelm I, Fazakas C and Krizbai IA. In vitro models of the blood-brain barrier. Acta Neurobiol Exp (Wars). 2011;71:113-28.

184. Cucina A, Borrelli V, Randone B, Coluccia P, Sapienza P and Cavallaro A. Vascular endothelial growth factor increases the migration and proliferation of smooth muscle cells through the mediation of growth factors released by endothelial cells. J Surg Res. 2003;109:16-23.

185. Kubes P, Suzuki M and Granger DN. Nitric oxide: an endogenous modulator of leukocyte adhesion. Proceedings of the National Academy of Sciences of the United States of America. 1991;88:4651-5.

186. Shu X, Keller TCt, Begandt D, Butcher JT, Biwer L, Keller AS, Columbus L and Isakson BE. Endothelial nitric oxide synthase in the microcirculation. Cellular and molecular life sciences : CMLS. 2015.

187. Forstermann U and Sessa WC. Nitric oxide synthases: regulation and function. European heart journal. 2012;33:829-37, 837a-837d.

188. Heiss C, Rodriguez-Mateos A and Kelm M. Central role of eNOS in the maintenance of endothelial homeostasis. Antioxidants \& redox signaling. 2015;22:123042.

189. Shesely EG, Maeda N, Kim HS, Desai KM, Krege JH, Laubach VE, Sherman PA, Sessa WC and Smithies O. Elevated blood pressures in mice lacking endothelial nitric oxide synthase. Proceedings of the National Academy of Sciences of the United States of America. 1996;93:13176-81.

190. Fulton D, Fontana J, Sowa G, Gratton JP, Lin M, Li KX, Michell B, Kemp BE, Rodman D and Sessa WC. Localization of endothelial nitric-oxide synthase phosphorylated on serine 1179 and nitric oxide in Golgi and plasma membrane defines 
the existence of two pools of active enzyme. The Journal of biological chemistry. 2002;277:4277-84.

191. van Haperen R, Cheng C, Mees BM, van Deel E, de Waard M, van Damme LC, van

Gent T, van Aken T, Krams R, Duncker DJ and de Crom R. Functional expression of endothelial nitric oxide synthase fused to green fluorescent protein in transgenic mice.

The American journal of pathology. 2003;163:1677-86.

192. Shaul PW, Smart EJ, Robinson LJ, German Z, Yuhanna IS, Ying Y, Anderson RG and Michel T. Acylation targets emdothelial nitric-oxide synthase to plasmalemmal caveolae.

The Journal of biological chemistry. 1996;271:6518-22.

193. Ju H, Zou R, Venema VJ and Venema RC. Direct interaction of endothelial nitricoxide synthase and caveolin-1 inhibits synthase activity. The Journal of biological chemistry. 1997;272:18522-5.

194. Michel JB, Feron O, Sacks D and Michel T. Reciprocal regulation of endothelial nitric-oxide synthase by Ca2+-calmodulin and caveolin. The Journal of biological chemistry. 1997;272:15583-6.

195. Looft-Wilson RC, Todd SE, Araj CA, Mutchler SM and Goodell CA. Alpha(1)adrenergic-mediated eNOS phosphorylation in intact arteries. Vascular pharmacology. 2013;58:112-7.

196. Igarashi J, Thatte HS, Prabhakar P, Golan DE and Michel T. Calcium-independent activation of endothelial nitric oxide synthase by ceramide. Proceedings of the National Academy of Sciences of the United States of America. 1999;96:12583-8.

197. Igarashi J, Bernier SG and Michel T. Sphingosine 1-phosphate and activation of endothelial nitric-oxide synthase. differential regulation of Akt and MAP kinase pathways by EDG and bradykinin receptors in vascular endothelial cells. The Journal of biological chemistry. 2001;276:12420-6.

198. Cantalupo A, Zhang Y, Kothiya M, Galvani S, Obinata H, Bucci M, Giordano FJ, Jiang XC, Hla T and Di Lorenzo A. Nogo-B regulates endothelial sphingolipid homeostasis to control vascular function and blood pressure. Nature medicine. 2015;21:1028-37. 199. Oancea $\mathrm{E}$ and Meyer T. Protein kinase $\mathrm{C}$ as a molecular machine for decoding calcium and diacylglycerol signals. Cell. 1998;95:307-18.

200. Lipp P and Reither G. Protein kinase C: the "masters" of calcium and lipid. Cold Spring Harbor perspectives in biology. 2011;3.

201. Mutchler SM and Straub AC. Compartmentalized nitric oxide signaling in the resistance vasculature. Nitric oxide : biology and chemistry / official journal of the Nitric Oxide Society. 2015;49:8-15.

202. Meens MJ, Alonso F, Le Gal L, Kwak BR and Haefliger JA. Endothelial Connexin37 and Connexin 40 participate in basal but not agonist-induced NO release. Cell communication and signaling : CCS. 2015;13:34.

203. Pfenniger A, Derouette JP, Verma V, Lin X, Foglia B, Coombs W, Roth I, Satta N, Dunoyer-Geindre S, Sorgen P, Taffet S, Kwak BR and Delmar M. Gap junction protein Cx37 interacts with endothelial nitric oxide synthase in endothelial cells. Arteriosclerosis, thrombosis, and vascular biology. 2010;30:827-34.

204. Alonso F, Boittin FX, Beny JL and Haefliger JA. Loss of connexin 40 is associated with decreased endothelium-dependent relaxations and eNOS levels in the mouse 
aorta. American journal of physiology Heart and circulatory physiology. 2010;299:H1365-73.

205. Mather S, Dora KA, Sandow SL, Winter P and Garland CJ. Rapid endothelial cellselective loading of connexin 40 antibody blocks endothelium-derived hyperpolarizing factor dilation in rat small mesenteric arteries. CircRes. 2005;97:399-407.

206. Iwakiri Y, Satoh A, Chatterjee S, Toomre DK, Chalouni CM, Fulton D, Groszmann

RJ, Shah VH and Sessa WC. Nitric oxide synthase generates nitric oxide locally to regulate compartmentalized protein S-nitrosylation and protein trafficking. Proceedings of the National Academy of Sciences of the United States of America. 2006;103:1977782.

207. Boo YC, Kim HJ, Song H, Fulton D, Sessa W and Jo H. Coordinated regulation of endothelial nitric oxide synthase activity by phosphorylation and subcellular localization. Free radical biology \& medicine. 2006;41:144-53.

208. Fleming I, Fisslthaler B, Dimmeler S, Kemp BE and Busse R. Phosphorylation of

$\mathrm{Thr}(495)$ regulates $\mathrm{Ca}(2+) /$ calmodulin-dependent endothelial nitric oxide synthase activity. Circulation research. 2001;88:E68-75.

209. Sim AT and Scott JD. Targeting of PKA, PKC and protein phosphatases to cellular microdomains. Cell calcium. 1999;26:209-17.

210. Rosse C, Linch M, Kermorgant S, Cameron AJ, Boeckeler K and Parker PJ. PKC and the control of localized signal dynamics. Nature reviews Molecular cell biology. 2010;11:103-12.

211. Wang L, Wu B, Sun Y, Xu T, Zhang X, Zhou M and Jiang W. Translocation of protein kinase $C$ isoforms is involved in propofol-induced endothelial nitric oxide synthase activation. British journal of anaesthesia. 2010;104:606-12.

212. Partovian C, Zhuang Z, Moodie K, Lin M, Ouchi N, Sessa WC, Walsh K and Simons M. PKCalpha activates eNOS and increases arterial blood flow in vivo. Circ Res. 2005;97:482-7.

213. Oubaha $M$ and Gratton JP. Phosphorylation of endothelial nitric oxide synthase by atypical PKC zeta contributes to angiopoietin-1-dependent inhibition of VEGFinduced endothelial permeability in vitro. Blood. 2009;114:3343-51.

214. Haines RJ, Corbin KD, Pendleton LC and Eichler DC. Protein kinase Calpha phosphorylates a novel argininosuccinate synthase site at serine 328 during calciumdependent stimulation of endothelial nitric-oxide synthase in vascular endothelial cells.

The Journal of biological chemistry. 2012;287:26168-76.

215. Xiao Z, Wang T, Qin H, Huang C, Feng Y and Xia Y. Endoplasmic reticulum Ca2+ release modulates endothelial nitric-oxide synthase via extracellular signal-regulated kinase (ERK) 1/2-mediated serine 635 phosphorylation. The Journal of biological chemistry. 2011;286:20100-8.

216. Dimmeler S, Fleming I, Fisslthaler B, Hermann C, Busse R and Zeiher AM. Activation of nitric oxide synthase in endothelial cells by Akt-dependent phosphorylation. Nature. 1999;399:601-605.

217. Fulton D, Gratton JP, McCabe TJ, Fontana J, Fujio Y, Walsh K, Franke TF, Papapetropoulos A and Sessa WC. Regulation of endothelium-derived nitric oxide production by the protein kinase Akt. Nature. 1999;399:597-601. 
218. Maxfield FR and van Meer G. Cholesterol, the central lipid of mammalian cells. Current opinion in cell biology. 2010;22:422-9.

219. $\mathrm{XuX}$ and London $\mathrm{E}$. The effect of sterol structure on membrane lipid domains reveals how cholesterol can induce lipid domain formation. Biochemistry. 2000;39:843-

9.

220. Miersch S, Espey MG, Chaube R, Akarca A, Tweten R, Ananvoranich S and Mutus

B. Plasma membrane cholesterol content affects nitric oxide diffusion dynamics and signaling. The Journal of biological chemistry. 2008;283:18513-21.

221. Beevers G, Lip GY and O'Brien E. ABC of hypertension: The pathophysiology of hypertension. BMJ. 2001;322:912-6.

222. Coffman TM. Under pressure: the search for the essential mechanisms of hypertension. Nat Med. 2011;17:1402-9.

223. Oparil S, Zaman MA and Calhoun DA. Pathogenesis of hypertension. Ann Intern Med. 2003;139:761-76.

224. Levy BI, Ambrosio G, Pries AR and Struijker-Boudier HA. Microcirculation in hypertension: a new target for treatment? Circulation. 2001;104:735-40.

225. Kerr PM, Tam R, Ondrusova K, Mittal R, Narang D, Tran CH, Welsh DG and Plane F. Endothelial feedback and the myoendothelial projection. Microcirculation. 2012;19:416-22.

226. Sandow SL and Hill CE. Incidence of myoendothelial gap junctions in the proximal and distal mesenteric arteries of the rat is suggestive of a role in endothelium-derived hyperpolarizing factor-mediated responses. Circ Res. 2000;86:341-6.

227. Boerman EM, Everhart JE and Segal SS. Advanced age decreases local calcium signaling in endothelium of mouse mesenteric arteries in vivo. Am J Physiol Heart Circ Physiol. 2016;310:H1091-6.

228. Gelebart $P$, Opas M and Michalak M. Calreticulin, a Ca2+-binding chaperone of the endoplasmic reticulum. Int J Biochem Cell Biol. 2005;37:260-6.

229. Arnaudeau S, Frieden M, Nakamura K, Castelbou C, Michalak M and Demaurex

N. Calreticulin differentially modulates calcium uptake and release in the endoplasmic reticulum and mitochondria. J Biol Chem. 2002;277:46696-705.

230. Liu N, Fine RE, Simons E and Johnson RJ. Decreasing calreticulin expression lowers the $\mathrm{Ca} 2+$ response to bradykinin and increases sensitivity to ionomycin in NG108-15 cells. J Biol Chem. 1994;269:28635-9.

231. Jamur MC and Oliver C. Permeabilization of cell membranes. Methods Mol Biol. 2010;588:63-6.

232. Schulz I. Permeabilizing cells: some methods and applications for the study of intracellular processes. Methods Enzymol. 1990;192:280-300.

233. Afshar N, Black BE and Paschal BM. Retrotranslocation of the chaperone calreticulin from the endoplasmic reticulum lumen to the cytosol. $\mathrm{Mol} \mathrm{Cell} \mathrm{Biol}$. 2005;25:8844-53.

234. Harvey A, Montezano AC and Touyz RM. Vascular biology of ageing-Implications in hypertension. J Mol Cell Cardiol. 2015;83:112-21. 
235. Zimmerman KA, Xing D, Pallero MA, Lu A, Ikawa M, Black L, Hoyt KL, Kabarowski $\mathrm{JH}$, Michalak $\mathrm{M}$ and Murphy-Ullrich JE. Calreticulin Regulates Neointima Formation and Collagen Deposition following Carotid Artery Ligation. J Vasc Res. 2015;52:306-20. 236. Xu FF, Tao TQ, Wang XR, Li YZ, Song DD, Liu M and Liu XH. Cytosolic calreticulin inhibits microwave radiation-induced microvascular endothelial cell injury through the integrin-focal adhesion kinase pathway. Microcirculation. 2014;21:717-29.

237. Clark RA, Li SL, Pearson DW, Leidal KG, Clark JR, Denning GM, Reddick R, Krause $\mathrm{KH}$ and Valente AJ. Regulation of calreticulin expression during induction of differentiation in human myeloid cells. Evidence for remodeling of the endoplasmic reticulum. J Biol Chem. 2002;277:32369-78.

238. Nakamura K, Bossy-Wetzel E, Burns K, Fadel MP, Lozyk M, Goping IS, Opas M, Bleackley RC, Green DR and Michalak M. Changes in endoplasmic reticulum luminal environment affect cell sensitivity to apoptosis. J Cell Biol. 2000;150:731-40.

239. Cannesson M, Jian Z, Chen G, Vu TQ and Hatib F. Effects of phenylephrine on cardiac output and venous return depend on the position of the heart on the Frank-

Starling relationship. J Appl Physiol (1985). 2012;113:281-9.

240. Coppolino M, Leung-Hagesteijn C, Dedhar S and Wilkins J. Inducible interaction of integrin alpha 2 beta 1 with calreticulin. Dependence on the activation state of the integrin. J Biol Chem. 1995;270:23132-8.

241. Rojiani MV, Finlay BB, Gray V and Dedhar S. In vitro interaction of a polypeptide homologous to human Ro/SS-A antigen (calreticulin) with a highly conserved amino acid sequence in the cytoplasmic domain of integrin alpha subunits. Biochemistry. 1991;30:9859-66.

242. Papp S, Fadel MP, Kim H, McCulloch CA and Opas M. Calreticulin affects fibronectin-based cell-substratum adhesion via the regulation of c-Src activity. J Biol Chem. 2007;282:16585-98.

243. Papp S, Szabo E, Kim H, McCulloch CA and Opas M. Kinase-dependent adhesion to fibronectin: regulation by calreticulin. Exp Cell Res. 2008;314:1313-26.

244. Jackson WF, Boerman EM, Lange EJ, Lundback SS and Cohen KD. Smooth muscle alpha1D-adrenoceptors mediate phenylephrine-induced vasoconstriction and increases in endothelial cell Ca2+ in hamster cremaster arterioles. Br J Pharmacol. 2008;155:51424.

245. Faustino RS, Chiriac A, Niederlander NJ, Nelson TJ, Behfar A, Mishra PK, Macura $S$, Michalak M, Terzic A and Perez-Terzic C. Decoded calreticulin-deficient embryonic stem cell transcriptome resolves latent cardiophenotype. Stem Cells. 2010;28:1281-91. 246. Scheppke L, Murphy EA, Zarpellon A, Hofmann JJ, Merkulova A, Shields DJ, Weis SM, Byzova TV, Ruggeri ZM, Iruela-Arispe ML and Cheresh DA. Notch promotes vascular maturation by inducing integrin-mediated smooth muscle cell adhesion to the endothelial basement membrane. Blood. 2012;119:2149-58.

247. Szestakowska D SE, Eggleton P, Opas M, Young P. 7th International Workshop on Calreticulin, Niagara Falls, Canada

The Complexities of Calreticulin From Protein Folding to Disease Prevention and Therapeutic Application. Calcium Binding Proteins. 2006;1:135-139. 
248. Dey S and Matsunami H. Calreticulin chaperones regulate functional expression of vomeronasal type 2 pheromone receptors. Proc Natl Acad Sci U S A. 2011;108:166516.

249. Bernard-Marissal N, Moumen A, Sunyach C, Pellegrino C, Dudley K, Henderson CE, Raoul C and Pettmann B. Reduced calreticulin levels link endoplasmic reticulum stress and Fas-triggered cell death in motoneurons vulnerable to ALS. J Neurosci. 2012;32:4901-12.

250. Galan M, Kassan M, Kadowitz PJ, Trebak M, Belmadani S and Matrougui K. Mechanism of endoplasmic reticulum stress-induced vascular endothelial dysfunction. Biochim Biophys Acta. 2014;1843:1063-75.

251. Kassan M, Galan M, Partyka M, Saifudeen Z, Henrion D, Trebak M and Matrougui

$\mathrm{K}$. Endoplasmic reticulum stress is involved in cardiac damage and vascular endothelial dysfunction in hypertensive mice. Arterioscler Thromb Vasc Biol. 2012;32:1652-61.

252. Bertolotti A, Zhang Y, Hendershot LM, Harding HP and Ron D. Dynamic interaction of BiP and ER stress transducers in the unfolded-protein response. Nat Cell Biol. 2000;2:326-32.

253. Dora KA, Hinton JM, Walker SD and Garland CJ. An indirect influence of phenylephrine on the release of endothelium-derived vasodilators in rat small mesenteric artery. Br J Pharmacol. 2000;129:381-7.

254. Taylor MS, Bonev AD, Gross TP, Eckman DM, Brayden JE, Bond CT, Adelman JP and Nelson MT. Altered expression of small-conductance Ca2+-activated K+ (SK3) channels modulates arterial tone and blood pressure. Circ Res. 2003;93:124-31.

255. Si H, Heyken WT, Wolfle SE, Tysiac M, Schubert R, Grgic I, Vilianovich L, Giebing G, Maier T, Gross V, Bader M, de Wit C, Hoyer J and Kohler R. Impaired endotheliumderived hyperpolarizing factor-mediated dilations and increased blood pressure in mice deficient of the intermediate-conductance Ca2+-activated $\mathrm{K}+$ channel. Circ Res. 2006;99:537-44.

256. Strobaek D, Teuber L, Jorgensen TD, Ahring PK, Kjaer K, Hansen RS, Olesen SP, Christophersen P and Skaaning-Jensen B. Activation of human IK and SK Ca2+-activated $\mathrm{K}+$ channels by NS309 (6,7-dichloro-1H-indole-2,3-dione 3-oxime). Biochim Biophys Acta. 2004;1665:1-5.

257. Behringer EJ and Segal SS. Impact of Aging on Calcium Signaling and Membrane Potential in Endothelium of Resistance Arteries: A Role for Mitochondria. J Gerontol A Biol Sci Med Sci. 2017.

258. Ashour DJ, Pelka B, Jaaks P, Wundenberg T, Blechner C, Zobiak B, Failla AV and Windhorst $\mathrm{S}$. The catalytic domain of inositol-1,4,5-trisphosphate 3-kinase-a contributes to ITPKA-induced modulation of F-actin. Cytoskeleton (Hoboken). 2015;72:93-100. 259. Windhorst S, Minge D, Bahring R, Huser S, Schob C, Blechner C, Lin HY, Mayr GW and Kindler S. Inositol-1,4,5-trisphosphate 3-kinase A regulates dendritic morphology and shapes synaptic Ca2+ transients. Cell Signal. 2012;24:750-7.

260. Koster JD, Leggewie B, Blechner C, Brandt N, Fester L, Rune G, Schweizer M, Kindler $S$ and Windhorst S. Inositol-1,4,5-trisphosphate-3-kinase-A controls morphology of hippocampal dendritic spines. Cell Signal. 2016;28:83-90. 
261. Schell MJ, Erneux C and Irvine RF. Inositol 1,4,5-trisphosphate 3-kinase A associates with $\mathrm{F}$-actin and dendritic spines via its $\mathrm{N}$ terminus. J Biol Chem. 2001;276:37537-46.

262. Isakson BE, Best AK and Duling BR. Incidence of protein on actin bridges between endothelium and smooth muscle in arterioles demonstrates heterogeneous connexin expression and phosphorylation. Am J Physiol Heart Circ Physiol. 2008;294:H2898-904.

263. Morris AP, Gallacher DV, Irvine RF and Petersen OH. Synergism of inositol trisphosphate and tetrakisphosphate in activating Ca2+-dependent $\mathrm{K}+$ channels. Nature. 1987;330:653-5.

264. Luckhoff A and Clapham DE. Inositol 1,3,4,5-tetrakisphosphate activates an endothelial Ca(2+)-permeable channel. Nature. 1992;355:356-8.

265. Erickson RR, Dunning LM and Holtzman JL. The effect of aging on the chaperone concentrations in the hepatic, endoplasmic reticulum of male rats: the possible role of protein misfolding due to the loss of chaperones in the decline in physiological function seen with age. J Gerontol A Biol Sci Med Sci. 2006;61:435-43.

266. Paz Gavilan M, Vela J, Castano A, Ramos B, del Rio JC, Vitorica J and Ruano D.

Cellular environment facilitates protein accumulation in aged rat hippocampus.

Neurobiol Aging. 2006;27:973-82.

267. Hedhli N, Huang Q, Kalinowski A, Palmeri M, Hu X, Russell RR and Russell KS. Endothelium-derived neuregulin protects the heart against ischemic injury. Circulation. 2011;123:2254-62.

268. Odiete O, Hill MF and Sawyer DB. Neuregulin in cardiovascular development and disease. Circ Res. 2012;111:1376-85.

269. Lemmens K, Segers VF, Demolder M and De Keulenaer GW. Role of neuregulin1/ErbB2 signaling in endothelium-cardiomyocyte cross-talk. J Biol Chem. 2006;281:19469-77.

270. Kwon MS, Park CS, Choi K, Ahnn J, Kim JI, Eom SH, Kaufman SJ and Song WK. Calreticulin couples calcium release and calcium influx in integrin-mediated calcium signaling. Mol Biol Cell. 2000;11:1433-43.

271. Socha MJ, Boerman EM, Behringer EJ, Shaw RL, Domeier TL and Segal SS. Advanced age protects microvascular endothelium from aberrant $\mathrm{Ca}(2+)$ influx and cell death induced by hydrogen peroxide. J Physiol. 2015;593:2155-69.

272. Beziau DM, Toussaint F, Blanchette A, Dayeh NR, Charbel C, Tardif JC, Dupuis J and Ledoux J. Expression of phosphoinositide-specific phospholipase $C$ isoforms in native endothelial cells. PLoS One. 2015;10:e0123769. 